\title{
Transformation and Effects of Silver and Copper Oxide Nanoparticles during Treatment and after Land Application of Sludge
}

\author{
by \\ Zainab Kadhim Abdulsada
}

A thesis submitted to the Faculty of Graduate and Postdoctoral Affairs in partial fulfillment of the requirements for the degree of

Doctor of Philosophy

in

Environmental Engineering

Carleton University

Ottawa, Ontario

(C) 2019 Zainab Kadhim Abdulsada 


\section{Abstract}

The increasing antimicrobial application of silver nanoparticles (AgNPs) and copper oxide nanoparticles (CuONPs) in industries has led to significant quantities being released into wastewater and sludge. This has raised significant concerns about their impact on soil biological activities after sludge land application. This thesis evaluates the transformation of AgNPs and CuONPs and their impact on the bacterial community during sludge treatment (anaerobic digestion, chemical conditioning, and lime stabilization) and subsequent land application. Land application of sludge was simulated in the laboratory using soil microcosms over 105 days. Bacterial population and diversity were assessed through examining specific phyla or the overall bacterial composition in the sludge and soil. The results showed that AgNPs in sludge went through dissolution, agglomeration, and surface coating. During sludge anaerobic digestion, AgNPs and CuONPs at 2, 10 and $30 \mathrm{mg} / \mathrm{g}$ TS sludge had no significant impact on the overall bacterial community structure at phylum and genus levels. AgNPs and CuONPs also did not significantly impact the biogas generation over time, except for sludge reactor that had higher concentration of CuONPs (30 mg NPs/g TS), which showed significant reduction in biogas generation over time. After applying the anaerobically digested sludge on soil, AgNPs at $20 \mathrm{mg} / \mathrm{kg}$ soil had no significant impact, while the concentrations of AgNPs at $300 \mathrm{mg} / \mathrm{kg}$ soil and CuONPs at 20 and $300 \mathrm{mg} / \mathrm{kg}$ soil decreased the average relative abundance of highly abundant phyla and genera, and lowered the population of the plant growth promoting rhizobacteria (PGPR) by 10.5, 18, 42 and 41\%, respectively, suggesting that CuONPs pose higher toxicity. Sludge chemical conditioning using ferric chloride, alum, and synthetic polymer efficiently removed AgNPs from the aqueous phase and concentrated them in sludge solid. 
Lime stabilization also removed AgNPs through association with lime molecules and sludge solids. The presence of AgNPs at $2 \mathrm{mg} / \mathrm{g}$ TS sludge showed no significant impact on the population of the phyla Acidobacteria, Actinobacteria, Bacteroidetes, Firmicutes, and Proteobacteria in soil that received untreated, conditioned or lime stabilized sludge. This thesis presents important findings that can serve as a foundation for risk assessment of AgNPs and CuONPs toxicity in soil and potentially plant systems. 


\section{Acknowledgements}

I received a great deal of wonderful assistance and support throughout my $\mathrm{PhD}$ journey.

First and foremost, my deepest gratitude goes to Allah and Ahlul-Bayt for the countless blessings in my life, now and forever.

I would like to sincerely thank my supervisor, Professor Banu Ormeci for her invaluable knowledge, monitoring, encouragement and constructive criticism, without which this study could not have been realized. Thank you for giving me this opportunity and ensuring that I followed all the relevant rules and requirements, as well as for contributing your time, ideas and support. I would also like to thank my co-supervisor, Professor Maria DeRosa for her scientific monitoring and support. Both of you provided excellent examples of how women can succeed as engineers, chemists and professors.

I am also grateful for the evaluation committee; Professor Cigdem Eskicioglu, Professor Bill Willmore, Professor Majid Sartaj, and Professor Paul J. Van Geel for examining this research, and for their time and contributions.

I would like to thank Environment and Climate Change Canada for their collaboration and support. I would also like to thank them and NSERC for funding this research. I would like to thank the Civil and Environmental Engineering department for giving me the opportunity to work as a TA and for providing me with the departmental scholarship as well.

I wish to express my appreciation to the Research Associate, Rich Kibbee for his great guidance and monitoring during my experimental lab work. He trained me in many 
microscopic biological testing scenarios, and we developed and validated several testing techniques, including next-generation sequencing with MinIon and qPCR protocols. As well, he was always available to provide scientific methodology and information, and to answer my countless questions.

The lab graduate administrator, Marie Tudoret also deserves my full appreciation for providing a healthy work environment, helping set up experiments and efficiently managing the procurement of required materials. In addition, I want to thank those who I got in touch with during my lab work for their understanding, sharing and collaboration.

To my parents, who raised me to love science, contributed moral and emotional support and prayed for my success and happiness, I thank you so much.

I am so grateful to my kind brothers and sisters and friends who motivated me throughout this challenge, wished me the best, and provided happy distractions when things got tough.

I am also so grateful to my husband for his exceptional love, motivation and unconditional support. Without your patience, understanding, and encouragement this study could not have been completed.

And to my children, the shining lights of my life, I thank you for your love and support, and for sometimes making this journey more challenging, thereby making me stronger. 


\section{Table of Contents}

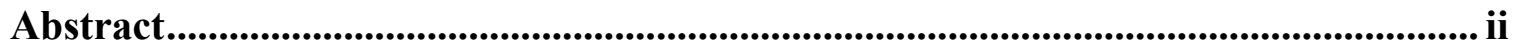

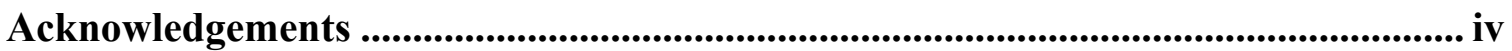

Table of Contents ............................................................................................... vi

List of Figures............................................................................................................... $\mathrm{xv}$

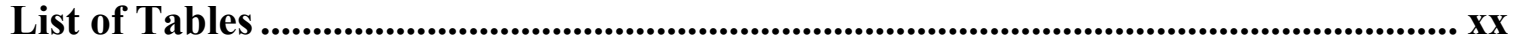

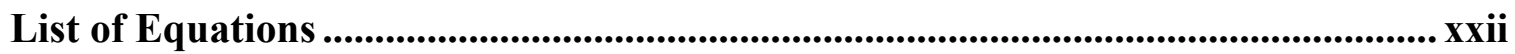

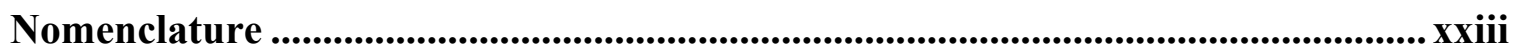

Chapter 1: Introduction .............................................................................................. 1

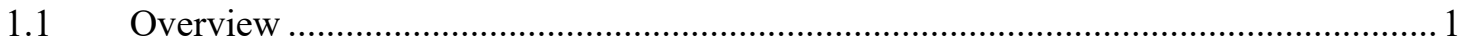

1.2 Objective of the study and research methodology .................................................. 4

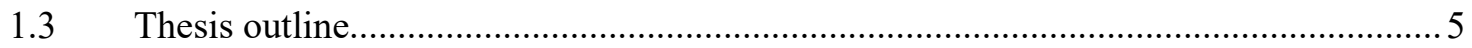

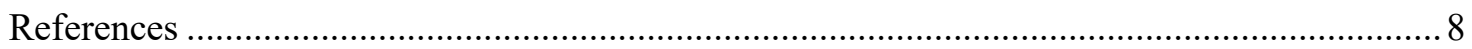

Chapter 2: Literature Review ................................................................................. 11

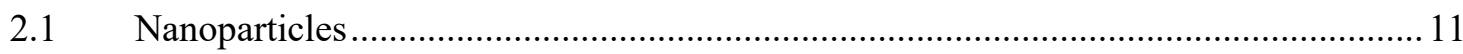

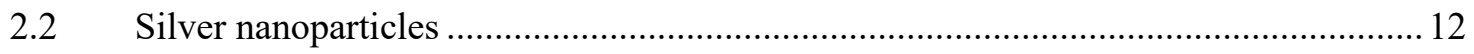

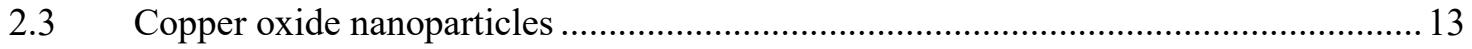

2.4 Public health and environmental impact of AgNPs and CuONPs............................. 14

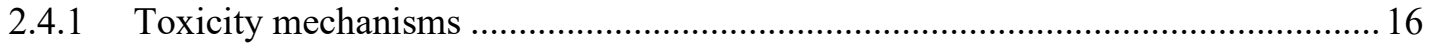

2.5 Transformation of AgNPs and CuONPs in the environment ................................... 18

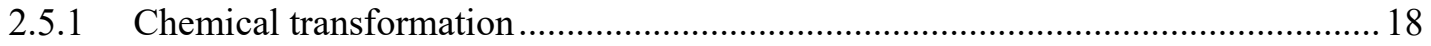

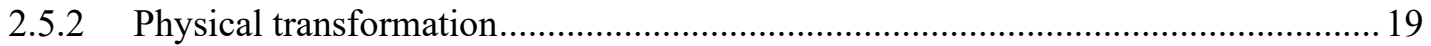

2.6 Parameters affecting the behavior and impact of nanoparticles ............................... 19

2.7 Mass flow of silver and copper into wastewater, sludge, and soil ...........................23 
2.8 Behavior and transformation of nanoparticle during wastewater and sludge treatment 25

2.9 Potential applications of nanomaterials in wastewater treatment

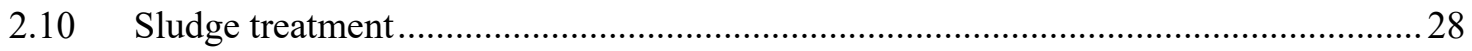

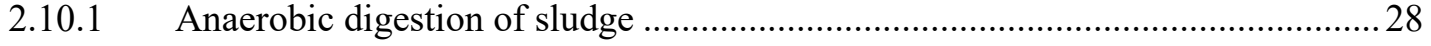

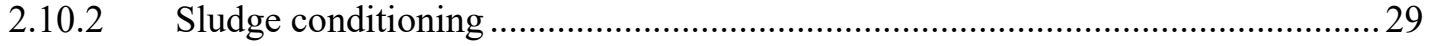

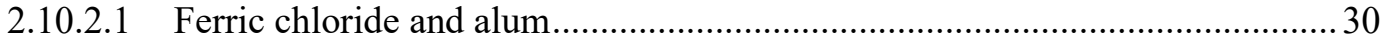

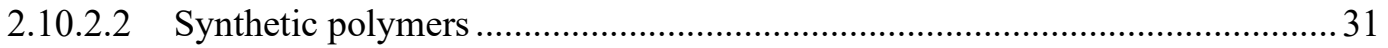

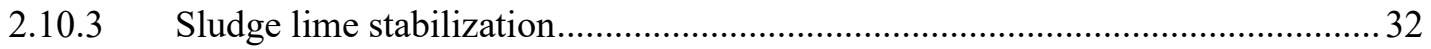

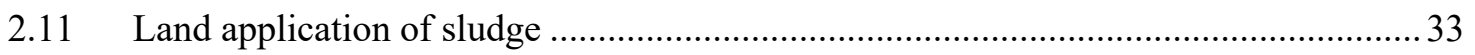

2.11.1 Impact of nanoparticles after land application of sludge ..................................... 33

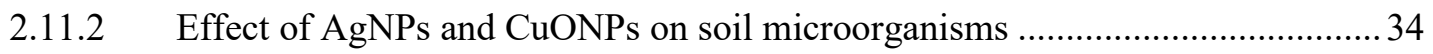

2.11.3 Influence of soil organic matter on AgNPs and CuONPs...................................35

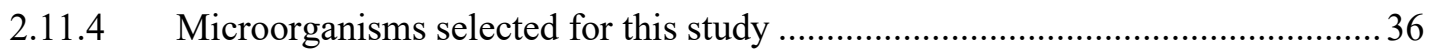

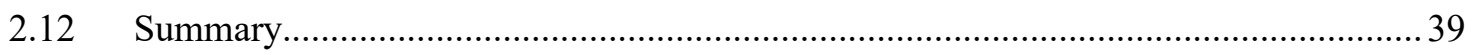

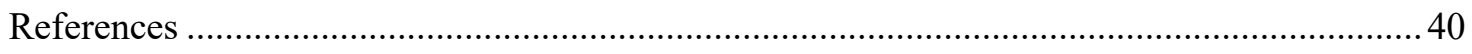

Chapter 3: Materials and Methods .................................................................. 53

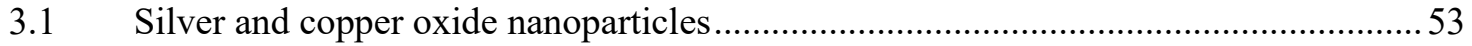

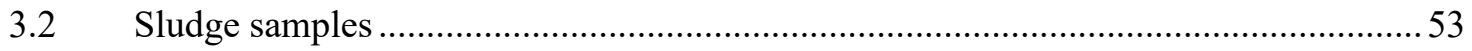

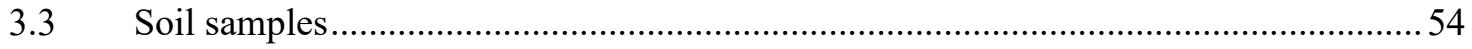

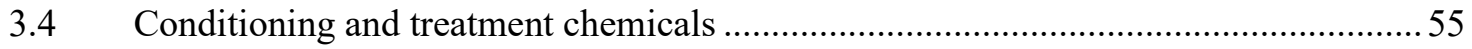

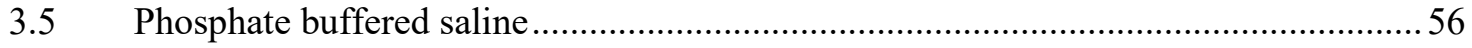

3.6 Quantification and characterization of nanoparticles .............................................56

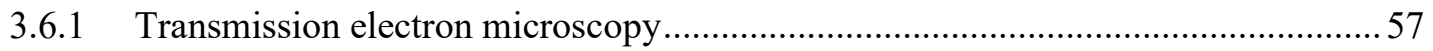

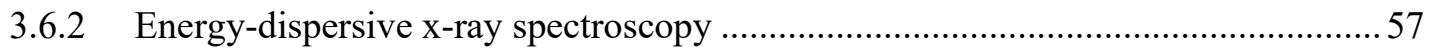

3.6.3 Single particle inductively coupled plasma mass spectrometry ….........................58 


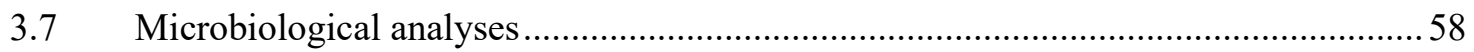

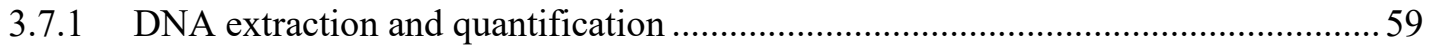

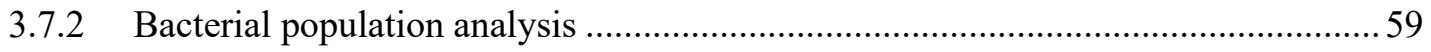

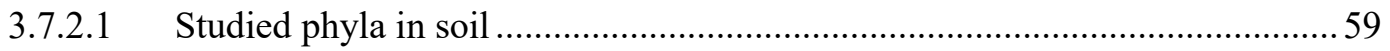

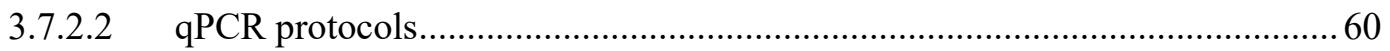

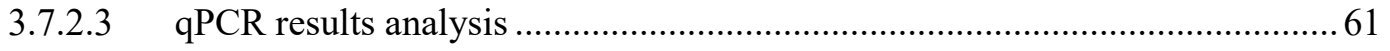

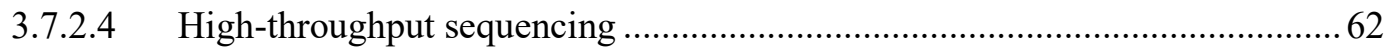

3.7.2.4.1 DNA extraction, quantification and quality control ................................63

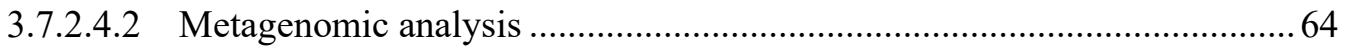

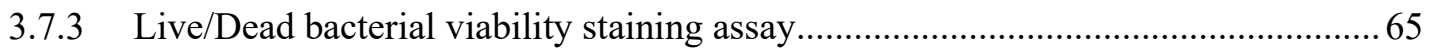

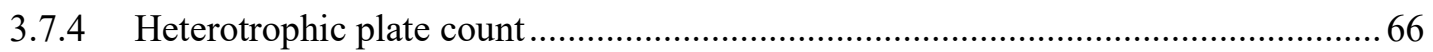

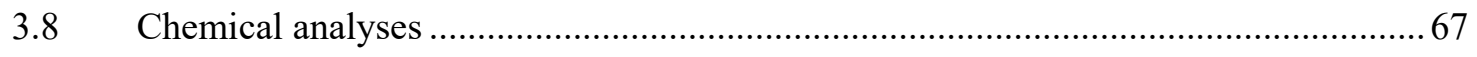

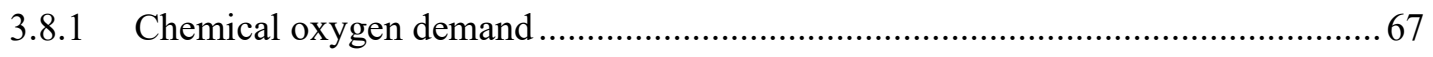

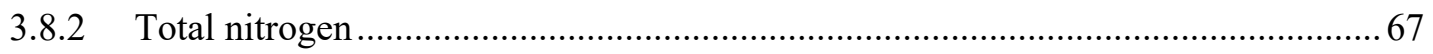

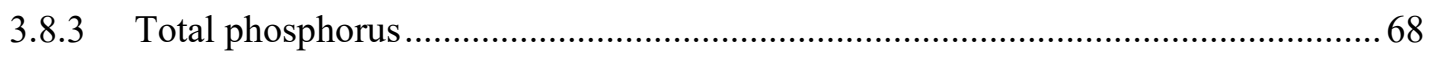

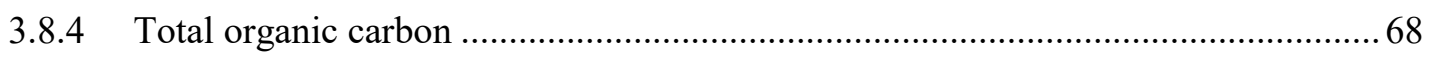

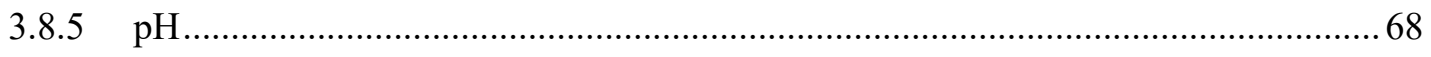

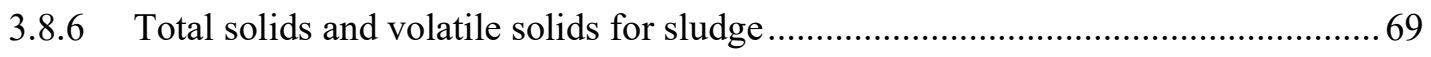

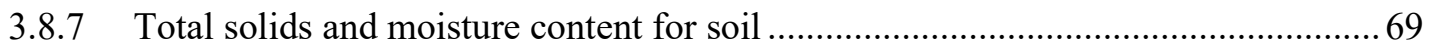

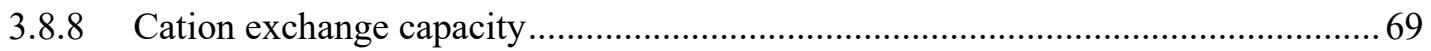

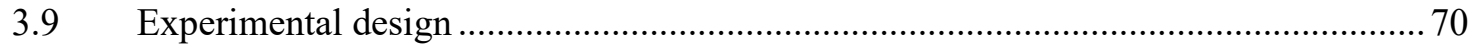

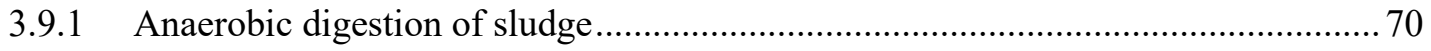

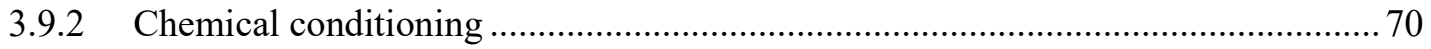

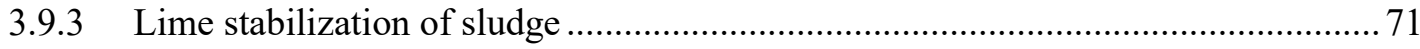

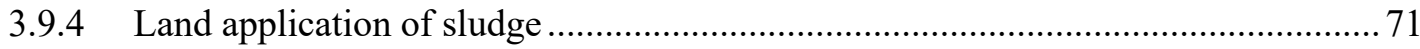

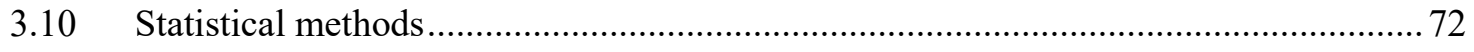




\section{Chapter 4: Impact of AgNPs and CuONPs on The Anaerobic Digestion of Sludge} and The Structure of Bacterial Community .......................................................... 76

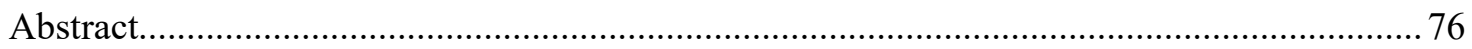

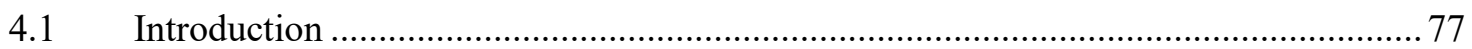

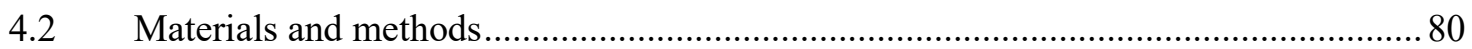

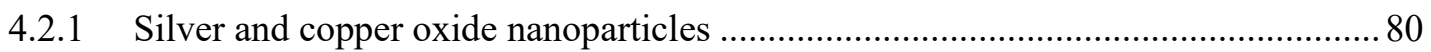

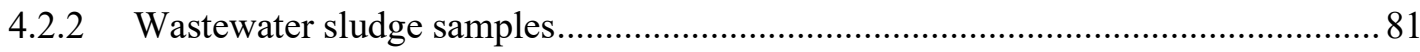

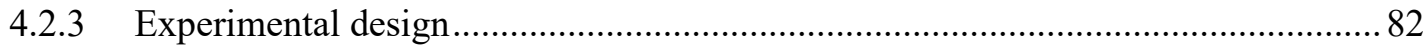

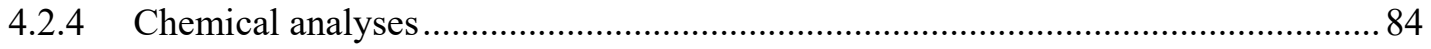

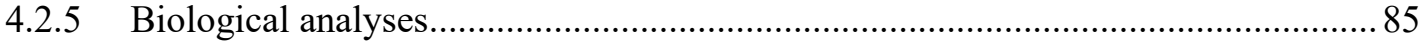

4.2.5.1 Live/Dead bacterial viability staining assay ................................................ 85

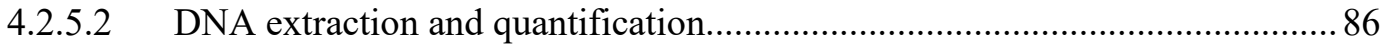

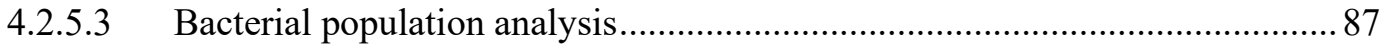

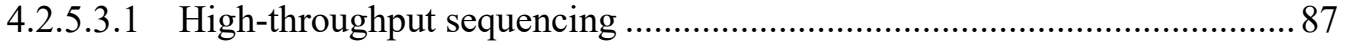

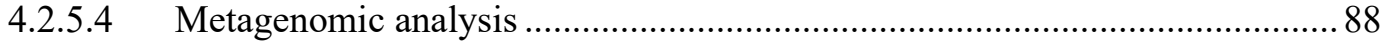

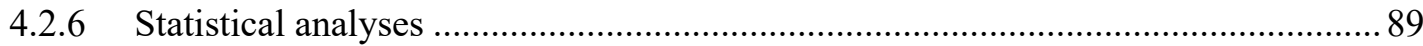

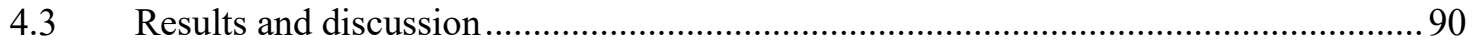

4.3.1 Chemical performance of the anaerobic digestion reactors .................................. 90

4.3.2 Impact of AgNPs and CuONPs on cell viability ................................................ 93

4.3.3 Bacterial community structure in the presence of AgNPs and CuONPs..................94

4.3.3.1 Abundance and diversity of bacteria at the phylum level............................. 98

4.3.3.2 Abundance and diversity of bacteria at the genus level .............................. 102

4.3.3.3 Key bacteria involved in the anaerobic digestion process ............................ 105

4.3.3.4 Metagenomic analysis of select genera .................................................... 110 
4.3.4 Discussion of the relationship between bacterial function and chemical performance of the reactors 113

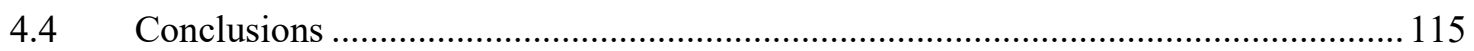

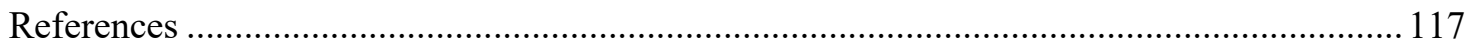

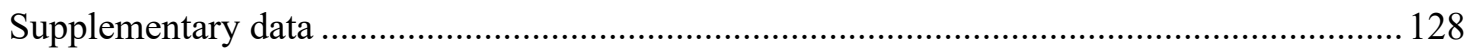

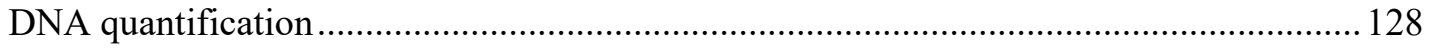

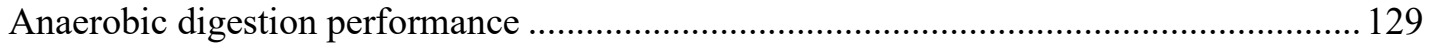

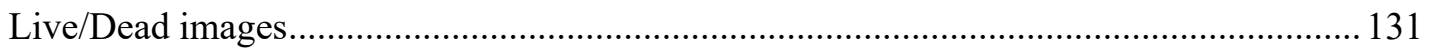

Genus of interest and their important role in the sludge anaerobic digestion .................... 138

\section{Chapter 5: Impact and Toxicity of AgNPs and CuONPs on Soil Bacterial}

Community Structure after Land Application of Anaerobically Digested Sludge. 141

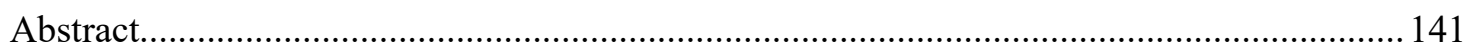

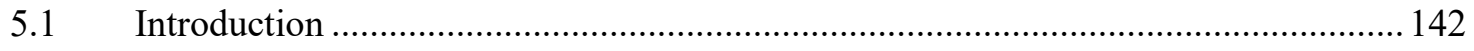

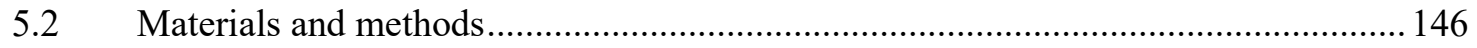

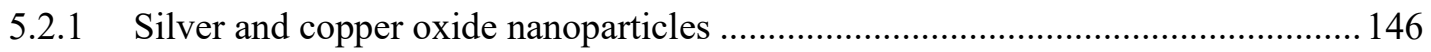

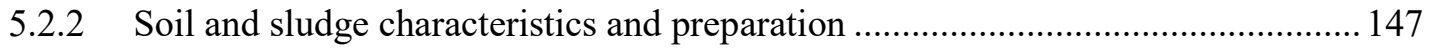

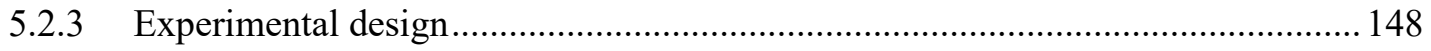

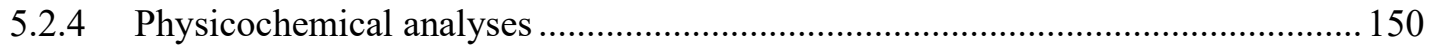

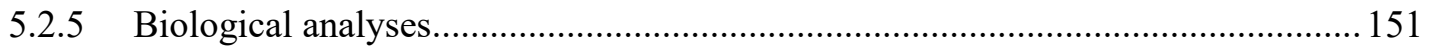

5.2.5.1 Live/Dead bacterial viability staining assay ............................................. 151

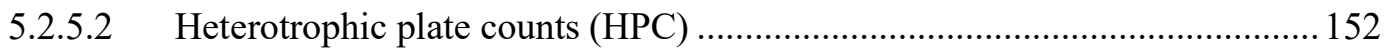

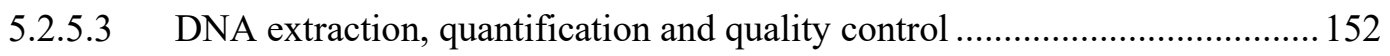

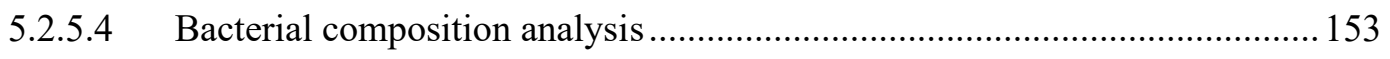

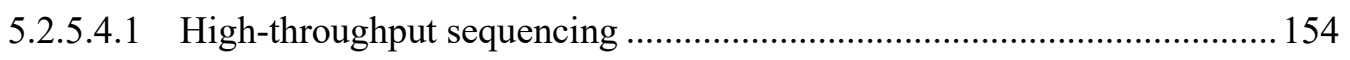

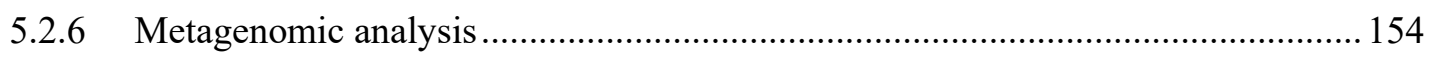

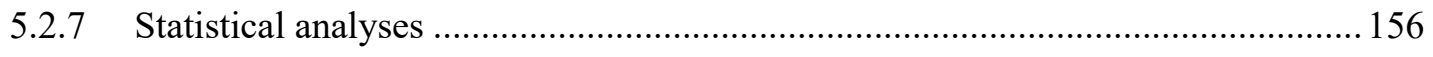


5.3 Results and discussion

5.3.1 Soil physicochemical properties.

5.3.2 Impact of AgNPs and CuONPs on the heterotrophic bacteria and percentage of live cells 158

5.3.3 Impact of AgNPs and CuONPs on bacterial community structure 160

5.3.3.1 Abundance and diversity of bacterial phyla ................................................. 164

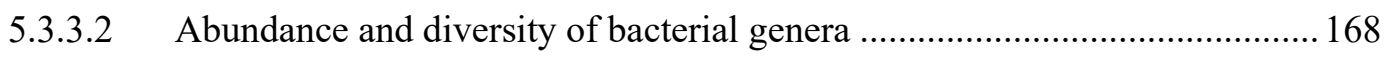

5.3.3.3 Key bacteria potentially involved in soil functions .................................... 172

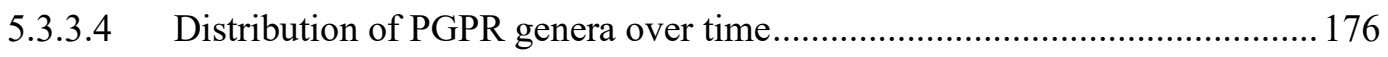

5.3.4 General discussion on the impact of AgNPs and CuONPs on soil biological activities 179

5.4 Conclusions 183

References . 185

Supplementary data

Total genomic DNA concentration

The genera of interest and their role in soil 198

Live/Dead cell images

\section{Chapter 6: Transformation and Removal of Silver Nanoparticles during Chemical Conditioning of Sludge and Their Impact on Soil Bacteria after Land Application}

\begin{tabular}{|c|c|}
\hline \multicolumn{2}{|r|}{ Abstract } \\
\hline 6.1 & 205 \\
\hline 6.2 & 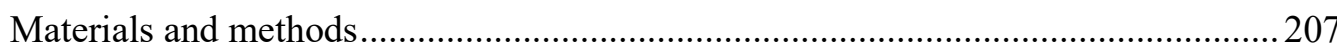 \\
\hline 6.2 .1 & AgNPs, sludge and soil characteristics and preparation.... \\
\hline 6.2 .2 & 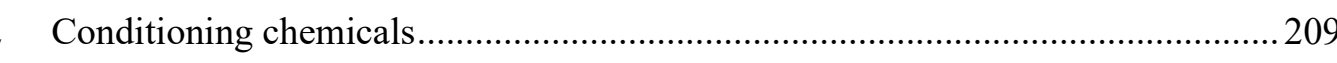 \\
\hline 6.2 .3 & 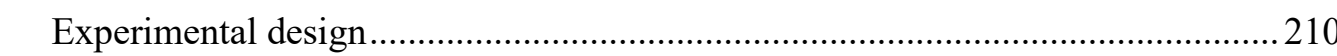 \\
\hline
\end{tabular}




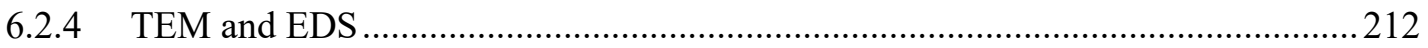

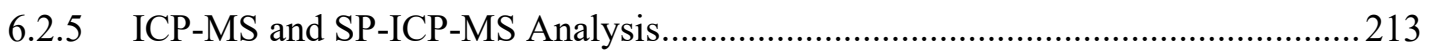

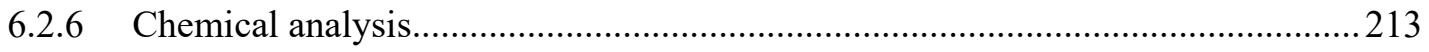

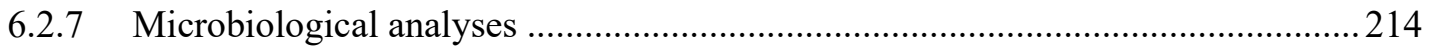

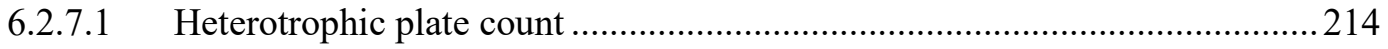

6.2.7.2 Live/Dead bacterial viability staining assay ............................................. 215

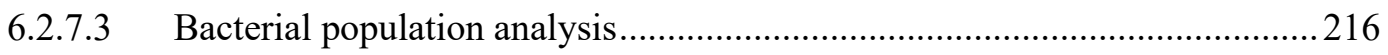

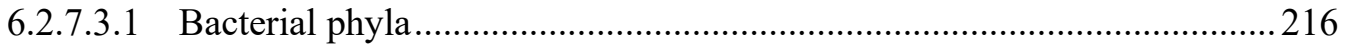

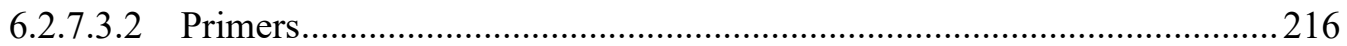

6.2.7.3.3 qPCR protocols and annealing temperature optimization .......................2217

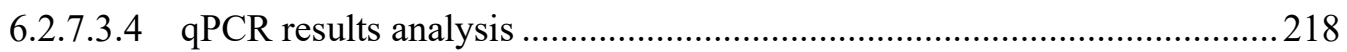

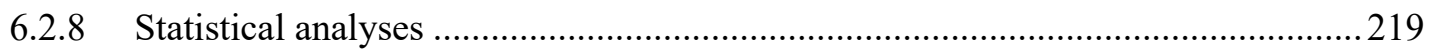

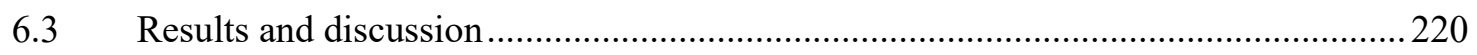

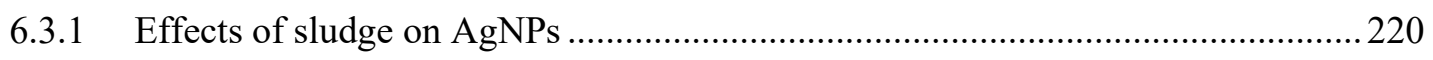

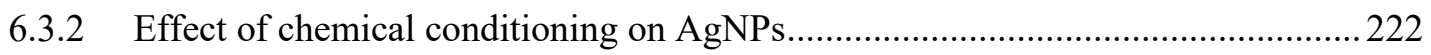

6.3.3 Structural analyses of AgNPs under different conditioning chemicals...................224

6.3.4 Impact of AgNPs after land application of biosolids .......................................228

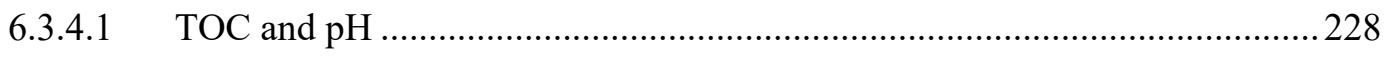

6.3.4.2 Impact of AgNPs on heterotrophic bacteria and Live/Dead viability ...........230

6.3.4.3 Impact of AgNPs on selected soil microorganisms....................................2231

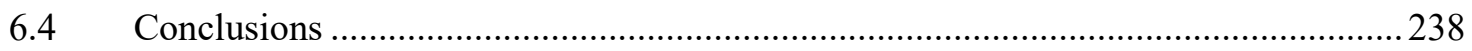

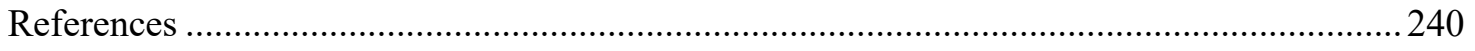

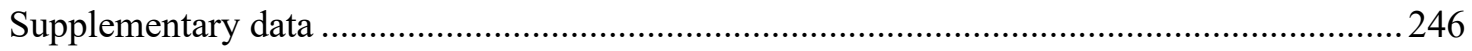

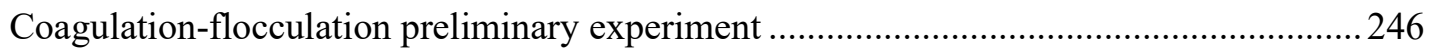

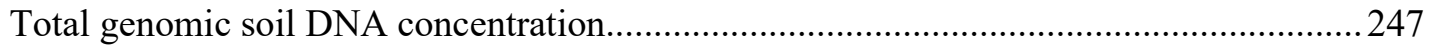

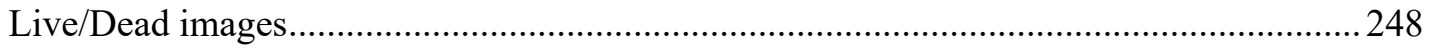




\section{Chapter 7: Transformation of Silver Nanoparticles (AgNPs) during Lime}

Treatment of Sludge and their Impact on Soil Bacteria after Land Application ... 253

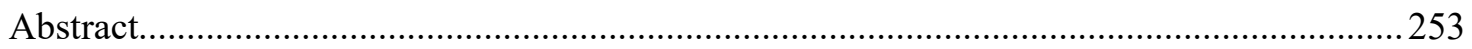

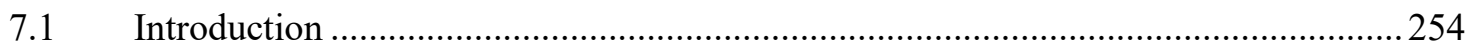

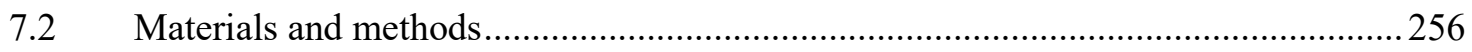

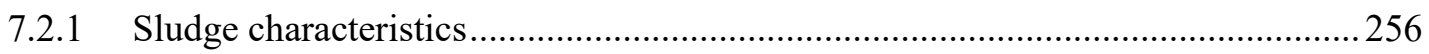

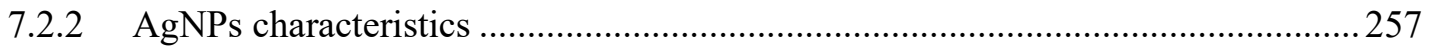

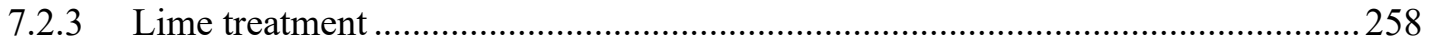

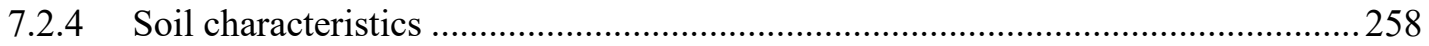

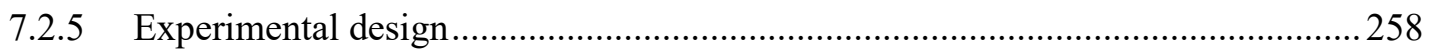

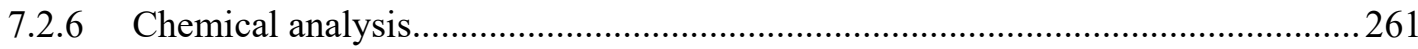

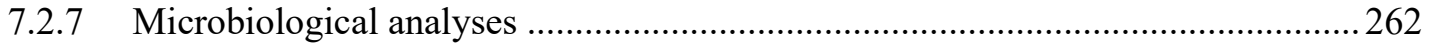

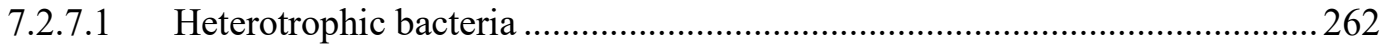

7.2.7.2 Live/Dead bacterial viability staining assay ……………………………....... 262

7.2.7.3 Bacterial population analysis......................................................................2. 263

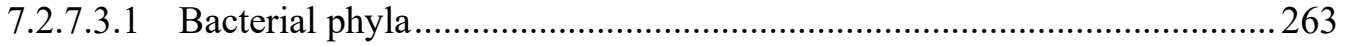

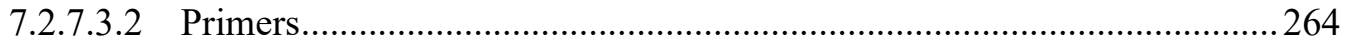

7.2.7.3.3 qPCR protocols and annealing temperature optimization ..........................264

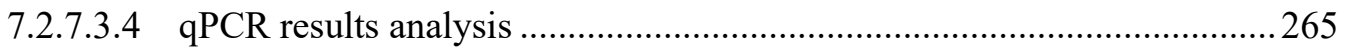

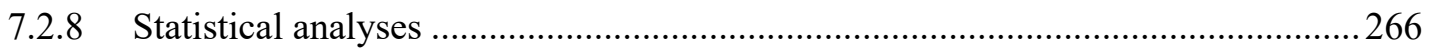

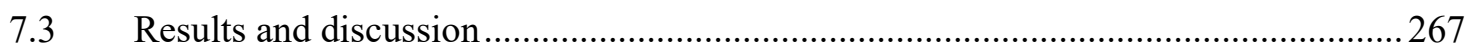

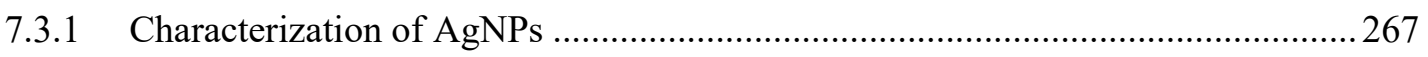

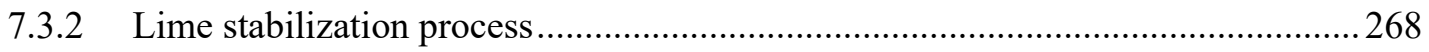

7.3.3 Land application of lime stabilized sludge............................................................2

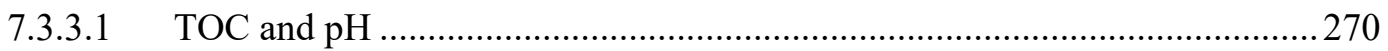

7.3.3.2 Impact of AgNPs on heterotrophic bacteria and Live/Dead viability ...........272 


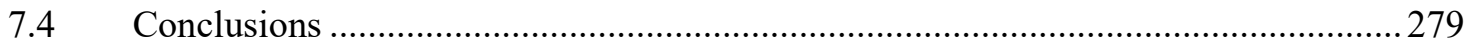

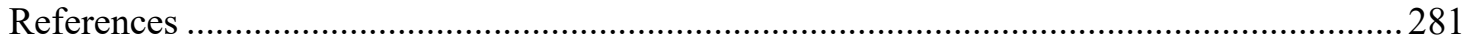

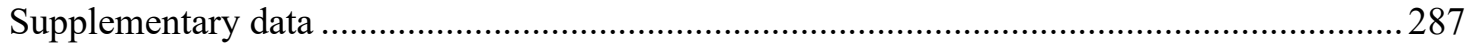

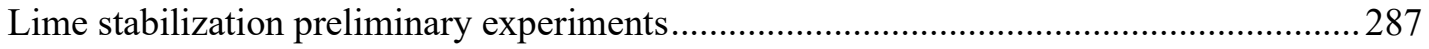

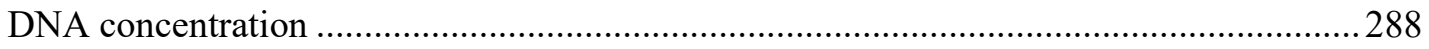

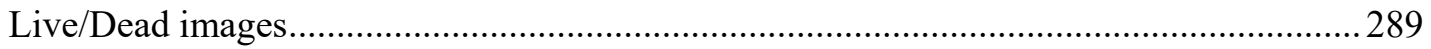

Chapter 8: Conclusions and Future Work ..................................................................... 292

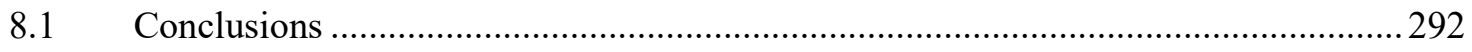

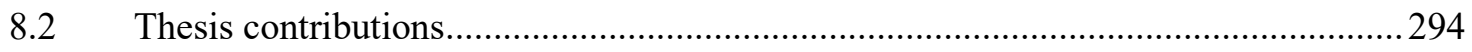

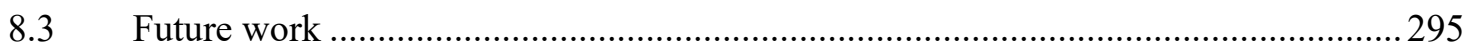




\section{List of Figures}

Figure 4-1 Cumulative biogas production over time for BMP reactors that had AgNPs (a) and CuONPs (b) with the control. The result showed that reactor $\mathrm{F}$ is significantly different from the control (p-value $=0.006)$ 92

Figure 4-2 Percent of live cells (based on Live/Dead bacterial viability staining of the sludge) for the control and reactors $\mathrm{A}, \mathrm{B}$, and $\mathrm{C}(\mathrm{a})$, and reactors $\mathrm{D}, \mathrm{E}$, and $\mathrm{F}$ (b) (the control had no nanoparticles and reactors $\mathrm{A}, \mathrm{B}$, and $\mathrm{C}$ had 2,10 , and $30 \mathrm{mg} \mathrm{AgNPs} / \mathrm{g} \mathrm{TS}$, respectively, and reactors $\mathrm{D}, \mathrm{E}$, and $\mathrm{F}$ had 2, 10, and $30 \mathrm{mg} \mathrm{CuONPs/g} \mathrm{TS}$, respectively). .94

Figure 4-3 Shannon-Wiener index for the control and reactors A, B, and C (a), and reactors D, E, and $\mathrm{F}$ (b) (the control had no nanoparticles and reactors A, B, and C had 2, 10, and $30 \mathrm{mg} \mathrm{AgNPs} / \mathrm{g}$ $\mathrm{TS}$, respectively, and reactors $\mathrm{D}, \mathrm{E}$, and $\mathrm{F}$ had 2, 10, and $30 \mathrm{mg} \mathrm{CuONPs} / \mathrm{g} \mathrm{TS}$, respectively).... 96

Figure 4-4 Overall phyla evenness (a) and phyla richness (represented by the number of the phyla whose relative abundance $\geq 0.01 \%$ ) (b) in the control and each reactor (reactors $\mathrm{A}, \mathrm{B}$, and $\mathrm{C}$ had 2 , 10, and $30 \mathrm{mg} \mathrm{AgNPs/g} \mathrm{TS,} \mathrm{respectively,} \mathrm{and} \mathrm{reactors} \mathrm{D,} \mathrm{E,} \mathrm{and} \mathrm{F} \mathrm{had} \mathrm{2,} \mathrm{10,} \mathrm{and} 30 \mathrm{mg} \mathrm{CuONPs} / \mathrm{g}$ $\mathrm{TS}$, respectively).

Figure 4-5 Beta-Euclidean diversity, representing the bacterial composition difference between the control and other reactors (reactors A, B, and C had 2, 10, and $30 \mathrm{mg}$ AgNPs/g TS, respectively, and reactors $\mathrm{D}, \mathrm{E}$, and $\mathrm{F}$ had 2, 10, and $30 \mathrm{mg}$ CuONPs/g TS, respectively)...... 98

Figure 4-6 Average relative abundance at the phylum level in each sludge BMP reactor. The most abundance phyla $\geq 98 \%$ of the observed OTUs (a), and the next most abundant bacterial phyla $\leq$ $2 \%$ of the observed OTUs (b). Phyla with $\leq 0.01 \%$ relative abundance were not included (reactors A, B, and C had 2, 10 and $30 \mathrm{mg} \mathrm{AgNPs/g} \mathrm{TS,} \mathrm{respectively,} \mathrm{and} \mathrm{reactors} \mathrm{D,} \mathrm{E,} \mathrm{and} \mathrm{F} \mathrm{had} \mathrm{2,} 10$ and $30 \mathrm{mg} \mathrm{CuONPs} / \mathrm{g} \mathrm{TS}$, respectively)

Figure 4-7 OTU prevalence over time (1), and total OTUs (2) of Bacteroidetes (a), Lentisphaerae (b), Verrucomicrobia (c), and Planctomycete (d) for each reactor (reactors A, B, and C had 2, 10, and $30 \mathrm{mg} \mathrm{AgNPs} / \mathrm{g} \mathrm{TS}$, respectively, and reactors D, E, and F had 2, 10, and $30 \mathrm{mg} \mathrm{CuONPs/g}$ $\mathrm{TS}$, respectively). 101 
Figure 4-8 Relative abundance of the top 21 genera and those that have a relative abundance $\geq 1 \%$ (reactors A, B and C had 2, 10 and $30 \mathrm{mg} \mathrm{AgNPs/g} \mathrm{TS,} \mathrm{respectively,} \mathrm{and} \mathrm{reactors} \mathrm{D,} \mathrm{E} \mathrm{and} \mathrm{F} \mathrm{had}$ 2, 10 and $30 \mathrm{mg} \mathrm{CuONPs/g}$ TS, respectively). 103

Figure 4-9 Relative abundance of genera ( 37 genera with relative abundance $>0.1 \%$ ), that were negatively affected by the presence of the nanoparticles (reactors A, B and C had 2, 10 and $30 \mathrm{mg}$ AgNPs/g TS, respectively, and reactors $\mathrm{D}, \mathrm{E}$ and $\mathrm{F}$ had 2, 10 and $30 \mathrm{mg}$ CuONPs/g TS, respectively). 104

Figure 4-10 Relative abundance of genera ( 37 genera with relative abundance $>0.1 \%$ ), that had higher observed OTUs in the presence of higher nanoparticle concentrations (reactors A, B and C had 2, 10 and $30 \mathrm{mg} \mathrm{AgNPs/g} \mathrm{TS,} \mathrm{respectively,} \mathrm{and} \mathrm{reactors} \mathrm{D,} \mathrm{E} \mathrm{and} \mathrm{F} \mathrm{had} \mathrm{2,} 10$ and $30 \mathrm{mg}$ $\mathrm{CuONPs} / \mathrm{g} \mathrm{TS}$, respectively). 104

Figure 4-11 Relative abundance of bacterial genera involved in hydrolysis, fermentation, and hydrolysis and fermentation (reactors A, B and C had 2, 10, and $30 \mathrm{mg} \mathrm{AgNPs/g} \mathrm{TS,} \mathrm{respectively,}$ and reactors $\mathrm{D}, \mathrm{E}$, and $\mathrm{F}$ had 2, 10, and $30 \mathrm{mg} \mathrm{CuONPs} / \mathrm{g} \mathrm{TS}$, respectively) 108

Figure 4-12 Relative abundance of bacterial genera involved in acetogenesis, fermentation and acetogenesis, and hydrolysis, fermentation, and acetogenesis (reactors A, B, and C had 2, 10, and $30 \mathrm{mg} \mathrm{AgNPs} / \mathrm{g}$ TS, respectively, and reactors D, E, and F had 2, 10, and $30 \mathrm{mg} \mathrm{CuONPs/g} \mathrm{TS,}$ respectively).

Figure 4-13 Trends of select genera over time for each BMP sludge reactor (reactors A, B, and C had 2, 10, and $30 \mathrm{mg} \mathrm{AgNPs/g} \mathrm{TS,} \mathrm{respectively,} \mathrm{and} \mathrm{reactors} \mathrm{D,} \mathrm{E,} \mathrm{and} \mathrm{F} \mathrm{had} \mathrm{2,} \mathrm{10,} \mathrm{and} 30 \mathrm{mg}$ $\mathrm{CuONPs} / \mathrm{g} \mathrm{TS}$, respectively).

Figure 4-13 (Continued). 113

Figure 5-1 Soil concentration of TOC (a) and $\mathrm{pH}$ (b) over time (Reactors A, B, C, and D are the soil microcosms, which received anaerobically digested sludge with $2 \mathrm{mg}$ AgNPs/g TS, $30 \mathrm{mg}$ AgNPs/g TS, 2 mg CuONPs/g TS, and 30 mg CuONPs /g TS, respectively). 158

Figure 5-2 Impact of AgNPs and CuONPs on soil microbial growth as measured by the mean \pm standard error of CFU derived from the heterotrophic plate counts (a), and \% of live cells derived from Live/Dead staining assay (b) (Reactors A, B, C, and D are the soil microcosms, which received 
anaerobically digested sludge with $2 \mathrm{mg}$ AgNPs/g TS, $30 \mathrm{mg} \mathrm{AgNPs/g} \mathrm{TS,} 2 \mathrm{mg}$ CuONPs/g TS, and $30 \mathrm{mg} \mathrm{CuONPs} / \mathrm{g} \mathrm{TS}$, respectively).

Figure 5-3 Shannon-Wiener index for each soil microcosm over time. Reactors A, B, C, and D are soil microcosms, which received anaerobically digested sludge with $2 \mathrm{mg}$ AgNPs/g TS, $30 \mathrm{mg}$ AgNPs/g TS, $2 \mathrm{mg} \mathrm{CuONPs/g} \mathrm{TS}$, and $30 \mathrm{mg} \mathrm{CuONPs/g} \mathrm{TS,} \mathrm{respectively.}$ 162

Figure 5-4 Overall phyla evenness (a) and phyla richness, represented by the number of phyla, whose relative abundance $\geq 0.01$ (b) in each reactor. Reactors A, B, C, and D are soil microcosms, which received anaerobically digested sludge with $2 \mathrm{mg}$ AgNPs/g TS, $30 \mathrm{mg}$ AgNPs/g TS, $2 \mathrm{mg}$ $\mathrm{CuONPs} / \mathrm{g} \mathrm{TS}$, and $30 \mathrm{mg} \mathrm{CuONPs} / \mathrm{g} \mathrm{TS}$, respectively.

Figure 5-5 Beta-Euclidean diversity, representing the bacterial composition difference between each reactor to all other reactors. Reactors A, B, C, and D are soil microcosms, which received anaerobically digested sludge with $2 \mathrm{mg}$ AgNPs/g TS, $30 \mathrm{mg}$ AgNPs/g TS, $2 \mathrm{mg} \mathrm{CuONPs} / \mathrm{g}$ TS, and $30 \mathrm{mg} \mathrm{CuONPs} / \mathrm{g} \mathrm{TS}$, respectively 164

Figure 5-6 Average relative abundance at the phylum level in each soil microcosm. Most abundance phyla $\geq 90 \%$ of the observed OTUs (a), and next most abundant bacterial phyla $\leq 10 \%$ of the observed OTUs (b). Reactors A, B, C, and D are soil microcosms, which received anaerobically digested sludge with $2 \mathrm{mg} \mathrm{AgNPs/g} \mathrm{TS,} 30 \mathrm{mg} \mathrm{AgNPs/g} \mathrm{TS,} 2 \mathrm{mg} \mathrm{CuONPs/g} \mathrm{TS}$, and $30 \mathrm{mg}$ CuONPs /g TS, respectively. 165

Figure 5-7 OTUs of phyla over time. Reactors A, B, C, and D are soil microcosms, which received anaerobically digested sludge with $2 \mathrm{mg}$ AgNPs/g TS, $30 \mathrm{mg} \mathrm{AgNPs} / \mathrm{g}$ TS, 2 mg CuONPs/g TS, and $30 \mathrm{mg} \mathrm{CuONPs} / \mathrm{g} \mathrm{TS}$, respectively.

Figure 5-7 (Continued) 168

Figure 5-8 The relative abundance of the top 18 genera (each genus with $\geq 1 \%$ relative in all soil reactors), and others representing 61 genera with a relative abundance of $0.1-1 \%$. Reactors $\mathrm{A}, \mathrm{B}$, $\mathrm{C}$, and D are soil microcosms, which received anaerobically digested sludge with $2 \mathrm{mg} \mathrm{AgNPs/g}$ TS, 30 mg AgNPs/g TS, 2 mg CuONPs/g TS, and 30 mg CuONPs/g TS, respectively.

Figure 5-9 The average relative abundance of genera that decreased as a result of AgNPs and CuONPs presence; genera $\geq 1$ (a) and genera 0.1-1 (b). Reactors A, B, C, and D are soil 
microcosms, which received anaerobically digested sludge with $2 \mathrm{mg} \mathrm{AgNPs} / \mathrm{g}$ TS, $30 \mathrm{mg} \mathrm{AgNPs/g}$ $\mathrm{TS}, 2 \mathrm{mg} \mathrm{CuONPs} / \mathrm{g} \mathrm{TS}$, and $30 \mathrm{mg} \mathrm{CuONPs} / \mathrm{g} \mathrm{TS}$, respectively 170

Figure 5-10 The average relative abundance of genera whose population increased as a result of the presence of AgNPs and CuONPs compared to the control. Reactors A, B, C, and D are soil microcosms, which received anaerobically digested sludge with $2 \mathrm{mg} \mathrm{AgNPs} / \mathrm{g}$ TS, $30 \mathrm{mg} \mathrm{AgNPs/g}$ TS, $2 \mathrm{mg} \mathrm{CuONPs/g} \mathrm{TS,} \mathrm{and} 30 \mathrm{mg} \mathrm{CuONPs} / \mathrm{g}$ TS, respectively. 171

Figure 5-11 Distribution of the genera Lysobacter (a), Xanthomonas (b), Bacillus (c) and Herbaspirillum (d) over time. Reactors A, B, C, and D are soil microcosms, which received anaerobically digested sludge with $2 \mathrm{mg}$ AgNPs/g TS, $30 \mathrm{mg}$ AgNPs/g TS, 2 mg CuONPs/g TS, and $30 \mathrm{mg}$ CuONPs /g TS, respectively.

Figure 6-1 TEM image with its EDS profile (elemental composition) of AgNPs suspended in water (a) and sludge (b).

Figure 6-2 TEM images show ferric chloride treatment effect on AgNPs suspended in water (a) and sludge (b), 1 and 2 refer to different scales. 226

Figure 6-3 TEM images show alum treatment effect on AgNPs suspended in water (a) and sludge (b), 1 and 2 refer to different scales. 226

Figure 6-4 TEM images show polymer treatment effect on AgNPs suspended in water (a) and sludge (b), 1 and 2 refer to different scales. 227

Figure 6-5 Soil TOC concentration (a) and $\mathrm{pH}(\mathrm{b})$ over time. 229

Figure 6-6 HPC, represented by CFU (a) and the percentage of live bacterial cells (b) in soil reactors over time. .231

Figure 6-7 The overall average relative abundance of bacterial phyla in all the soil reactors.....232

Figure 6-8 Relative change in CCE for the five phyla assessed in controls 1 and 2 over time. ..233

Figure 6-9 Relative change in CCE for Acidobacteria (a), Actinobacteria (b), Bacteroidetes (c), Firmicutes (d), Proteobacteria (e), in soil reactors over time 236

Figure 7-1 TEM image of AgNPs suspended in water with its EDS profile. 267 
Figure 7-2 TEM images show lime treatment effect on AgNPs suspended in nanopure water, with EDS profile. (a) and (b) refer to different scales.

Figure 7-3 TEM images show lime treatment effect on AgNPs suspended in sludge, with EDS profile. (a) and (b) refer to different scales.

Figure 7-4 Total organic carbon concentration (a) and $\mathrm{pH}$ (b), over time. Control 1 is the soil reactor that received untreated sludge with no nanoparticles, control 2 is the soil reactor that received untreated sludge with $2 \mathrm{mg} \mathrm{AgNPs/g} \mathrm{TS} \mathrm{sludge,} \mathrm{and} \mathrm{lime} \mathrm{is} \mathrm{the} \mathrm{soil} \mathrm{reactor} \mathrm{that} \mathrm{received} \mathrm{lime}$ treated sludge with $2 \mathrm{mg}$ AgNPs/g TS sludge. .272

Figure 7-5 HPC, represented by CFU (a) and the percentage of live cells in soil reactors (b) over time. Control 1 is the soil reactor that received untreated sludge with no nanoparticles, control 2 is the soil reactor that received untreated sludge with $2 \mathrm{mg} \mathrm{AgNPs/g}$ TS sludge, and lime is the soil reactor that received lime treated sludge with $2 \mathrm{mg} \mathrm{AgNPs/g} \mathrm{TS} \mathrm{sludge.}$

Figure 7-6 Relative change in CCE for Acidobacteria (a), Actinobacteria (b), Bacteroidetes (c), Firmicutes (d) and Proteobacteria (e). Control 1 is the soil reactor that received untreated sludge with no nanoparticles, control 2 is the soil reactor that received untreated sludge with $2 \mathrm{mg} \mathrm{AgNPs} / \mathrm{g}$ TS sludge, and lime is the soil reactor that received lime treated sludge with $2 \mathrm{mg}$ AgNPs/g TS sludge. 277 


\section{List of Tables}

Table 2-1 Studied phyla and their population and important action in the soil.

Table 3-1 AgNPs and CuONPs characteristics.

Table 3-2 Characteristics of raw sludge samples.

Table 3-3 Soil sample characteristics.

Table 3-4 Phyla primer pairs used in qPCR assay.

Table 3-5 Copy number of 16S rRNA in each bacterial genome (Větrovský \& Baldrian, 2013). 62

Table 3-6 Coagulation-flocculation experiment.

Table 4-1 AgNPs and CuONPs characteristics.

Table 4-2 Characterization of the three types of raw wastewater sludge

Table 4-3 BMP reactors characterizations and conditions. .84

Table 4-4 Biogas yield, tCOD, sCOD, TS, and VS reduction in the BMP reactors with and without AgNPs and CuONPs.

Table 4-5 Overall diversity of the microbial population in the BMP reactors (all time points combined). .96

Table 4-6 Relative abundance of bacterial genera involved in the different functional stages of the anaerobic digestion process (control had no nanoparticles and reactors $\mathrm{A}, \mathrm{B}$, and $\mathrm{C}$ had 2, 10, and $30 \mathrm{mg} \mathrm{AgNPs} / \mathrm{g} \mathrm{TS}$, respectively, and reactors D, E, and F had 2, 10, and $30 \mathrm{mg} \mathrm{CuONPs} / \mathrm{g}$ TS, respectively). 106

Table 5-1 AgNPs and CuONPs characteristics.

Table 5-2 Characterization of the three types of raw wastewater sludge. 148

Table 5-3 MinION sequencing statistics and Shannon-Wiener index for the soil microcosms (all time points combined) 
Table 5-4 PGPR and their relative abundance in each soil reactor.

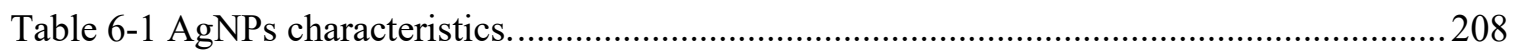

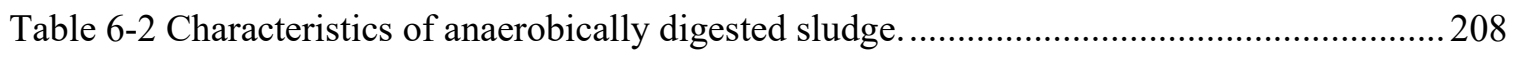

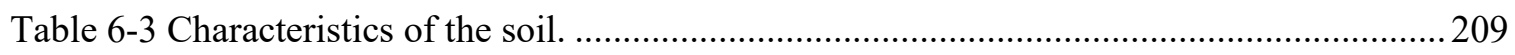

Table 6-4 Concentrations of conditioners and final $\mathrm{pH}$ values in water and sludge reactors......212

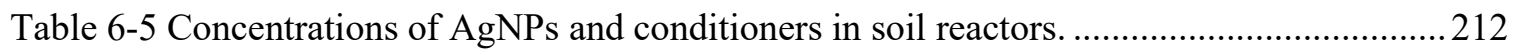

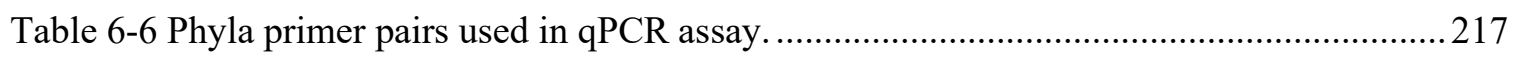

Table 6-7 Copy number of 16S rRNA in each bacterial genome (Větrovský \& Baldrian, 2013).

Table 6-8 Total, particulate, and dissolved silver concentrations in conditioned and control nanopure water and sludge samples measured by SP-ICP-MS and ICP-MS analysis................. 224

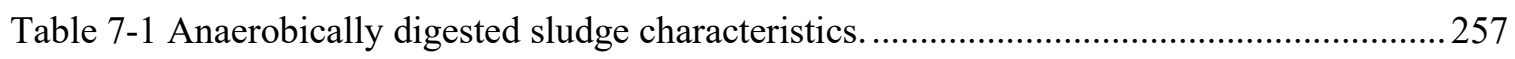

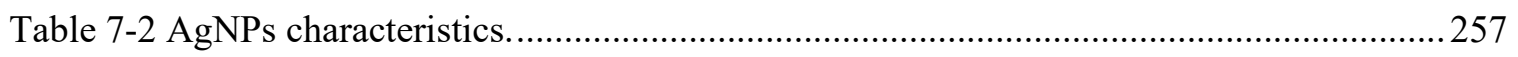

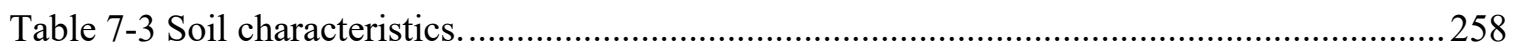

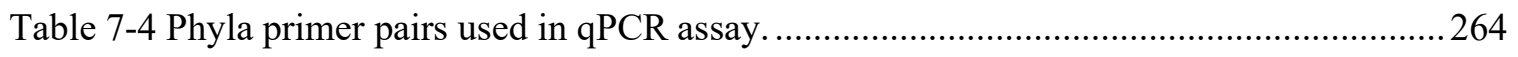

Table 7-5 Copy number of 16S rRNA in each bacterial genome (Větrovský \& Baldrian, 2013). 


\section{List of Equations}

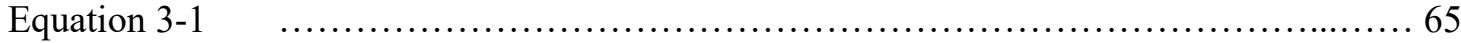

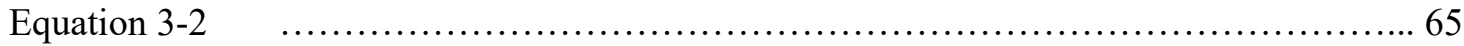

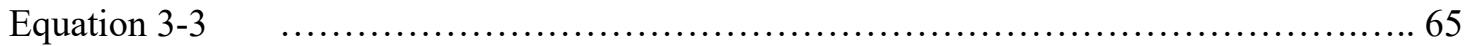

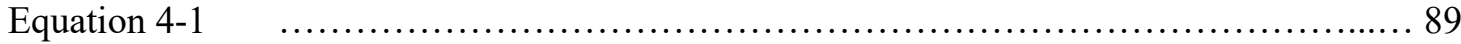

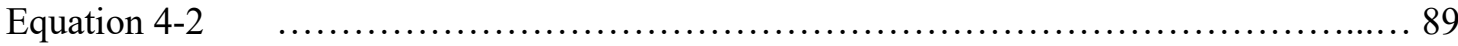

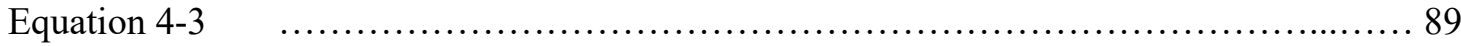

Equation $5-1 \quad$............................................................. 156

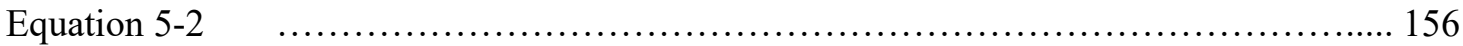

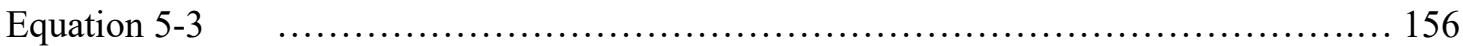




\section{Nomenclature}

\begin{tabular}{|c|c|}
\hline AgNPs & Silver nanoparticles \\
\hline ANOVA & Analysis of variance \\
\hline BMP & Biochemical methane potential \\
\hline $\mathrm{CCE}$ & Calculated CFU (colony forming unit) equivalent \\
\hline CEC & Cation exchange capacity \\
\hline $\mathrm{CFU}$ & Colony forming unit \\
\hline COD & Chemical oxygen demand \\
\hline $\mathrm{Cq}$ & Quantitation cycle \\
\hline CST & Capillary section time \\
\hline CuNPs & Copper nanoparticles \\
\hline CuONPs & Copper oxide nanoparticles \\
\hline $\mathrm{CV}$ & Coefficient of variance \\
\hline DL & Detective level \\
\hline DNA & Deoxyribonucleic acid \\
\hline EDS & Energy dispersive X-ray spectroscopy \\
\hline ENPs & Engineer nanoparticles \\
\hline EPS & Extracellular polymeric substances \\
\hline FOV & Field of view \\
\hline HPC & Heterotrophic plate count \\
\hline ICP-MS & Inductively coupled plasma mass spectrometry \\
\hline $\mathrm{MC}$ & Moisture content \\
\hline NPs & nanoparticles \\
\hline OTUs & Operational taxonomic units \\
\hline PBS & Phosphate buffered saline \\
\hline PGPR & Plant growth promoting rhizobacteria \\
\hline PVP & Polyvinylpyrrolidone \\
\hline qPCR & Quantitative polymerase chain reaction \\
\hline ROS & Reactive oxygen species \\
\hline rRNA & Ribosomal ribonucleic acid \\
\hline sCOD & Soluble Chemical oxygen demand \\
\hline
\end{tabular}




\section{Nomenclature (continued)}

$\begin{array}{ll}\text { SEM } & \text { Scanning electron microscopy } \\ \text { SP-ICP-MS } & \text { Single particle inductively coupled plasma mass spectrometry } \\ \text { tCOD } & \text { Total Chemical oxygen demand } \\ \text { TEM } & \text { Transmission electron microscopy } \\ \text { TN } & \text { Total nitrogen } \\ \text { TOC } & \text { Total organic carbon } \\ \text { TP } & \text { Total phosphorus } \\ \text { TS } & \text { Total solids } \\ \text { TSA } & \text { Tryptic soy agar } \\ \text { tWAS } & \text { Thickened waste activated sludge } \\ \text { VFAs } & \text { Volatile fatty acids } \\ \text { VS } & \text { Volatile solid }\end{array}$




\section{Chapter 1: Introduction}

\subsection{Overview}

Nanotechnology helps develop, improve, and innovate many daily consumer products through the use of engineering nanoparticles. Nanoparticles are entities with at least one dimension sized 1 to $100 \mathrm{~nm}$. They are specifically manufactured and designed by humans for many devices and material-related purposes. The small size of the nanoparticles enables them to have a large surface area to volume ratio, and thus, they have unique physicochemical properties. Thus, nanotechnology offers a variety of advantages, guarantees, risks, and wonders, which have grown significantly over the last few decades. It is utilized in many industries, including electronics, catalysis, cosmetics, textiles, medicine and pharmacy (Morris \& Willis, 2007; Rai et al., 2009; Mihranyan et al., 2012; de Francisco \& García-Estepa, 2018). However, recent studies have indicated that extensive use of nanoparticles leads to their presence in aqueous and terrestrial environments (Benn \& Westerhoff, 2008; Wiesner et al., 2009; Brar et al., 2010), and eventual interaction with ecosystem components such as organisms (Blaser et al., 2008). Although the expected concentration of released nanoparticles is very low, long-term exposure and accumulation is still a serious concern, as many studies have identified the toxic impact on organisms and environmental ecosystems. Therefore, it is crucial to understand the behavior of released nanoparticles, their transformation and the effects on ecosystems and living organisms. Due to their different antibacterial capabilities, silver nanoparticles (AgNPs) and copper oxide nanoparticles (CuONPs) are used in diverse applications. They are known to enhance the reactivity of materials and have been used in 
gas sensing, optoelectronics, catalysis, solar cells, pigments and paints, and antibacterial activity. However, the potential negative effects of both AgNPs and CuONPs are reported in the literature, and their rapidly increasing use has raised concerns about the quantities that end up in the environment.

There are several ecological and human health concerns of AgNPs and CuONPs since they have antibacterial effects. AgNPs and CuONPs can negatively affect other organisms, as has been reported in several studies (Dinesh et al., 2012; Zhang et al., 2016). However, most of these studies used nanoparticles spiked in pure cultures and did not consider the change in their characteristics due to various processes they undergo before reaching their destination.

AgNPs and CuONPs are released into the environment from many products, including textiles during laundering (Geranio et al., 2009; Benn \& Westerhoff, 2008), paints used in outdoor facades (Kaegi et al., 2010) and personal care and cosmetic products (Zhang et al., 2015). The majority of released nanoparticles are discharged into wastewater sewer systems and transported to wastewater treatment plants, which represent one of the main pathways for nanoparticles to be released into aquatic and terrestrial ecosystems (Brar et al., 2010; Geranio et al., 2009; Li et al., 2013). Wastewater treatment and sludge stabilization involve complex processes, and nanoparticles can interact with the organisms, organic matter and inorganic matter.

The main goals of wastewater treatment are to remove organic matter, nitrogen, and phosphorus. Nanoparticles go through several physical, chemical, and biological processes during wastewater treatment, and they can be removed by aggregation, settling, 
precipitation, and biosorption (Ganesh et al., 2010; Chen et al., 2012; Li et al., 2013). Wastewater sludge is a by-product of wastewater treatment, and it must be stabilized before disposal through different biological and chemical processes. The effect of AgNPs and CuONPs on biological wastewater treatment performance has been studied extensively (Chen et al., 2012; Ma et al., 2013; Kaegi et al., 2011). These studies showed the majority of discharged AgNPs and CuONPs were removed through bioadsorption and settling, which means nanoparticles will be mainly in sludge. Treated sludge is widely used as fertilizer, and nanoparticles are ultimately released into terrestrial ecosystems, where they are likely to interact with soil environments, including biological systems (Blaser et al., 2008).

Sludge stabilization is an essential process to eliminate contaminants and protect the environment and human health. Wastewater sludge is usually anaerobically digested, dewatered to 20 to $50 \%$ solids content, and further stabilized through the addition of lime and heat treatment or composting, before application on agricultural land (Metcalf \& Eddy, 2003). This process can potentially affect the behavior and interaction of AgNPs and CuONPs. In addition, it is critical to examine the potential impact of AgNPs and CuONPs on biological and chemical sludge treatment processes, as they are known to have negative effects on microorganisms and interact with other chemicals. As sludge is frequently applied to soil, its treatment is an important aspect of controlling the release of nanoparticles into the environment; thus, understanding their behavior, impact, and removal is very important. There is minimal data in the literature regarding the fate and impact of AgNPs or CuONPs during sludge treatment processes such as anaerobic 
digestion, lime stabilization, and chemical conditioning, and more importantly their impact on soil after land application of treated sludge.

Land application of biosolids is a sludge disposal option, which is beneficial to the soil. For example, from the estimated 780,000 dry tonnes of biosolids generated from 125 individual municipal wastewater treatment plants in ten Canadian provinces, approximately $53.4 \%$ is applied to soil as fertilizer (WRMD, 2017). Thus, land application of sludge is a major route of nanoparticles entering terrestrial ecosystems (Blaser et al., 2008; Mueller \& Nowack, 2008; Gottschalk et al., 2009). Several studies have shown the toxicity of $\mathrm{AgNPs}$ or $\mathrm{CuONPs}$ to soil microorganisms, and this is a concern since they play such an important role in maintaining healthy soil attributes such as organic matter decomposition and nutrient uptake and circulation. However, most available studies were conducted when AgNPs or CuONPs were directly spiked into the soil, which can result in different findings compared to real situations; thus, it is crucial to consider the impact of sludge treatment processes on $\mathrm{AgNPs}$ and CuONPs before land application.

\subsection{Objective of the study and research methodology}

The goal of this study was to investigate the transformation and effects of AgNPs and CuONPs during sludge chemical and biological treatment, and after application to soil. It is expected that assessing the impacts of AgNPs and CuONPs presence on sludge treatment and soil quality will result in new findings and contributions regarding the health and environmental impacts of nanoparticles. This was largely achieved by exploring the bacterial responses during different sludge treatments and after the application of treated sludge on soil. During the study, agricultural soil was treated with sludge that was subjected 
to chemical conditioning, lime stabilization, or anaerobic digestion. The objective of the study was met through the following activities:

1. An extensive literature review was conducted prior to the study and before each experiment, to scan the related work, to summarize the main findings and to build a strong knowledge base.

2. Experiments included controlled bench-scale sludge treatment and application to soil.

3. Several advanced analytical tools and techniques were incorporated to better examine and demonstrate nanoparticle behavior and impact, including:

- High-technology electron microscopy to visualize and image sludge samples below the nanometer scale; and,

- Molecular methods such as DNA isolation and quantification, Live/Dead viability staining, qPCR, and next-generation sequencing using MinIon.

4. Collecting, studying, and analyzing results, comparing them with other similar works and defining the main conclusions and recommendations.

\subsection{Thesis outline}

This thesis is constructed in a paper-based format, and it consists of a total of eight chapters as follow:

Chapter $\mathbf{1}$ is a brief general introduction to the research topics, including research objectives, methodology, and approach. The chapter is prepared for thesis purpose only.

Chapter $\mathbf{2}$ is a summary review of the literature that presents the relevant theoretical background information, as well as the previous works most related to the research. This chapter is prepared for the thesis purpose only. 
Chapter 3 provides a general description of the materials used and the experimental methods applied throughout the research. The chapter is prepared for thesis purposes only.

Chapters 4 to 7 are the primary research chapters of the thesis and are being prepared for submission as individual journal articles. My role and contribution were to conduct the experimental work (including preparing, running, and monitoring), analyze the data, and write the papers. I am the first author of the four papers, and the co-authors are my supervisor Professor Banu Ormeci, co-supervisor Professor Maria DeRosa, Rich Kibbee, who is a Research Associate, and a team from Environment and Climate Change Canada. I received training on using microbiology testing techniques and tools, such as DNA isolation and quantification, qPCR and Live/Dead viability staining, from Rich Kibbee, who also helped to develop and validate several testing techniques, including nextgeneration sequencing with MinIon which was used to analyze the DNA samples for bacterial population analysis in Chapters 4 and 5 .

Chapter 4 evaluated the impact and toxicity of AgNPs and CuONPs during sludge anaerobic digestion by studying the change in the overall sludge bacterial population and diversity during the process.

Chapter 5 studied the impact and toxicity of AgNPs and CuONPs on soil biological activity after land application of anaerobically digested sludge. This study investigated the change in the soil bacterial population and diversity over 105 days after applying the sludge.

Chapter 6 investigated the behavior and transformation of AgNPs during sludge chemical conditioning, as well as their impact after applying the treated sludge to soil. This study 
consisted of two stages. The sludge was first chemically conditioned, and AgNPs transformation was studied. Then treated sludge was applied to the soil, and the impact of AgNPs on soil biological activity was assessed by monitoring the soil characteristics over 105 days.

Chapter 7 investigated the behavior and transformation of AgNPs during sludge lime stabilization, and their impact after biosolids land application. This study also had two stages. First, sludge lime stabilization was performed, and AgNPs transformation was studied. Second, the stabilized sludge was applied to the soil, and the AgNPs impact on soil biological activity was examined by monitoring the soil over 105 days.

Chapter 8 provided the overall conclusions, significant findings and the final recommendation. The chapter is prepared for thesis purposes only.

Note: Since this thesis is in a paper-based format, it is important to note that some of the information in the introduction and materials and methods of the papers might be repeated. 


\section{References}

Benn, T. M., \& Westerhoff, P. (2008). Nanoparticle silver released into water from commercially available sock fabrics. Environmental Science \& Technology, 42(11), 4133-4139.

Blaser, S. A., Martin, S., Matthew, M., \& Konrad, H. (2008). Estimation of cumulative aquatic exposure and risk due to silver: contribution of nano-functionalized plastics and textiles. Science of The Total Environment, 390(2), 396-409.

Brar, S. K., Verma, M., Tyagi, R. D., \& Surampalli, R. Y. (2010). Engineered nanoparticles in wastewater and wastewater sludge-Evidence and impacts. Waste Management, 30(3), 504520.

Chen, Y., Wang, D., Zhu, X., Zheng, X., \& Feng, L. (2012). Long-term effects of copper nanoparticles on wastewater biological nutrient removal and $\mathrm{N} 2 \mathrm{O}$ generation in the activated sludge process. Environmental Science \& Technology, 46(22), 12452-12458.

de Francisco, E. V., \& García-Estepa, R. M. (2018). Nanotechnology in the agrofood industry. Journal of food engineering, 238, 1-11.

Dinesh, R., Anandaraj, M., Srinivasan, V., \& Hamza, S. (2012). Engineered nanoparticles in the soil and their potential implications to microbial activity. Geoderma, 173, 19-27.

Ganesh, R., Smeraldi, J., Hosseini, T., Khatib, L., Olson, B. H., \& Rosso, D. (2010). Evaluation of nanocopper removal and toxicity in municipal wastewaters. Environmental science \& technology, 44(20), 7808-7813.

Geranio, L., Manfred, H., \& Bernd, N. (2009). The behavior of silver nanotextiles during washing. Environmental Science \& Technology, 43(21), 8113-8118.

Gottschalk, F., Tobias, S., Roland W., S., \& Bernd, N. (2009). Modeled environmental concentrations of engineered nanomaterials ( $\mathrm{TiO} 2, \mathrm{ZnO}, \mathrm{Ag}, \mathrm{CNT}$, fullerenes) for different regions. Environmental Science \& Technology, 43(24), 9216-9222.

Kaegi, R., Sinnet, B., Zuleeg, S., Hagendorfer, H., Mueller, E., Vonbank, R., . . B Burkhardt, M. (2010). Release of silver nanoparticles from outdoor facades. Environmental pollution, 158(9), 2900-2905. 
Kaegi, R., Voegelin, A., Sinnet, B., Zuleeg, S., Hagendorfer, H., Burkhardt, M., \& Siegrist, H. (2011). Behavior of metallic silver nanoparticles in a pilot wastewater treatment plant. Environmental Science \& Technology, 45(9), 3902-3908.

Li, L., Hartmann, G., Döblinger, M., \& Schuster, M. (2013). uantification of nanoscale silver particles removal and release from municipal wastewater treatment plants in Germany. Environmental science \& technology, 47(13), 7317-7323.

Ma, R., Levard, C., Judy, J. D., Unrine, J. M., Durenkamp, M., Martin, B., . . Lowry, G. V. (2013). Fate of zinc oxide and silver nanoparticles in a pilot wastewater treatment plant and in processed biosolids. Environmental Science \& Technology, 48(1), 104-112.

Metcalf, L., \& Eddy, H. P. (2003). Waste Water Engineering: Treatment and Reuse (4 ed.). New York: McGraw-Hill.

Mihranyan, A., Ferraz, N., \& Strømme, M. (2012). Current status and future prospects of nanotechnology in cosmetics. Progress in materials science, 57(5), 875-910.

Morris, J., \& Willis, J. (2007). US Environmental Protection Agency nanotechnology white paper. Washington, DC: US Environmental Protection Agency.

Mueller, N. C., \& Nowack, B. (2008). Exposure modeling of engineered nanoparticles in the environment. Environmental Science and Technology, 42(12), 4447-4453.

Rai, M., Yadav, A., \& Gade, A. (2009). Silver nanoparticles as a new generation of antimicrobials. Biotechnology advances, 27(1), 76-83.

Wiesner, M. R., Lowry, G. V., Jones, K. L., Hochella, M. F., Giulio, R. T., Casman, E., \& Bernhardt, E. S. (2009). Decreasing uncertainties in assessing environmental exposure, risk, and ecological implications of nanomaterials. Environmental science and technology, $43,6458-6462$.

WRMD, E. C. (2017). Study of The Typical Management and Disposal Practices of Wastewater Treatment Sludge in Canada and Impacts on Municipal Solid Waste Landfills. Retrieved from https://buyandsell.gc.ca/procurement-data/award-notice/PW-16-00752877-001 
Zhang, W., Liu, X., Bao, S., Xiao, B., \& Fang, T. (2016). Evaluation of nano-specific toxicity of zinc oxide, copper oxide, and silver nanoparticles through toxic ratio. Journal of Nanoparticle Research, 18(12), 372.

Zhang, Y., Leu, Y.-R., Aitken, R. J., \& Riediker, M. (2015). Inventory of Engineered NanoparticleContaining ConsumerProducts Available in the Singapore Retail Market and Likelihood of Release into the Aquatic Environment. International Journal of Environmental Research and Public Health, 12(8), 8717-8743. 


\section{Chapter 2: Literature Review}

\subsection{Nanoparticles}

Nanoparticles are substances with at least one dimension between 1 to $100 \mathrm{~nm}$ in size. This unique size gives them a very large surface area to volume ratio and results in different electronic properties than conventional bulk counterparts. These properties make nanoparticles beneficial for many industrial functions, most importantly, antibacterial use. The growing interest in nanotechnology has led to increased worldwide production and application of nanoparticles in various industrial and commercial sectors, including the food, cosmetics, biomedical, pharmaceutical, electronics and energy industries (Morris \& Willis, 2007; Rai et al., 2009; Mihranyan et al., 2012). The study and development of nanoparticles began in the late twentieth century, after the invention of the atomic force microscope that could view fractions of a nanometer (Brar et al., 2010), and its use has expanded since then. In 2004, the estimated production of nanoparticles was 2,000 tons/year, and this was expected to increase to 58,000 tons/year from 2011 to 2020 (Nowack \& Bucheli, 2007). In 2009, there were 1,015 commercial products used by people that were manufactured with nanoparticles (Kim et al., 2012).

Nanoparticles can be released into the environment after the use of commercial and industrial products that were manufactured with nanoparticles (Wiesner et al., 2009; Brar et al., 2010; Benn \& Westerhoff, 2008). They have been detected in wastewater at significant concentrations, and sewer systems are considered the primary source for the release of nanoparticles into the environment (Brar et al., 2010; Geranio et al., 2009; Li et al., 2013). Recent research suggests that most nanoparticles discharged into wastewater are 
concentrated in sludge remaining after wastewater is passed through different wastewater treatment processes (Shafer et al., 1998, Kaegi et al., 2011, Li et al., 2016). The nanoparticles are eventually released into terrestrial ecosystems, and upon release, they can interact with soil environments, including the biological systems (Blaser et al., 2008). There is limited knowledge about the effects and transformation of nanoparticles after they are introduced into a sewer system, and during wastewater treatment, which involves a range of physical, chemical, and biological processes. Also, there is a significant knowledge gap in the literature about their effects on the environment and health, and their chronic toxicity after long-term exposure.

\subsection{Silver nanoparticles}

Silver nanoparticles (AgNPs) are commonly used as antibacterial agents and are thus incorporated into a wide range of products and applications. Many antimicrobial coatings on items, such as keyboards, wound care dressings, and biomedical devices, contain AgNPs that continuously release a low level of silver ions to protect against potentially harmful microorganisms (Lansdown, 2004; Cao \& Liu, 2010). In addition, AgNPs have unique optical, electrical, diagnostic, conductive and thermal properties, which are useful for applications such as photovoltaics, biological and chemical sensors and conductive inks (El-Nour et al., 2010; Larguinho \& Baptista, 2012; Babu et al., 2015).

The worldwide production of silver nanoparticles was estimated to be 452 ton/year in 2010 which resulted in $>200$ ton/year emission to wastewater and $>150$ ton/year emission to soil (Keller et al., 2013). Textile production is thought to be the most significant source of AgNPs in the environment, mainly due to the discharge of laundry water into the sewer 
system (Mueller \& Nowack, 2008). AgNPs can easily bleed out of the textiles in just a few wash cycles (Benn \& Westerhoff, 2008; Geranio et al., 2009). A recent study found that 34 to $85 \%$ of silver released from textiles is in the form of nanoparticles (Lorenz et al., 2012). These AgNPs will likely end up in wastewater treatment plants (Blaser et al., 2008;

Gottschalk et al., 2009). A significant fraction entering wastewater are accumulated in biosolids (sludge) at the end of treatment, which raises concerns about the effect of AgNPs on the soil after land application of biosolids (Shafer et al., 1998).

\subsection{Copper oxide nanoparticles}

Copper oxide nanoparticles (CuONPs) are used in many different industrial applications due to their distinctive properties. The small size $(\leq 100 \mathrm{~nm})$ gives them catalytic and antibacterial/fungal functions that are not present in commercial copper (particle size $>100$ $\mathrm{nm}$ ), and hence they behave differently than their bulk counterparts. The large catalytic surface area gives CuONPs very strong catalytic activity; they can achieve a higher reaction yield in a shorter time when used as a reagent in organic and organometallic synthesis (Dhas et al., 1998). In addition, their small size and larger surface area to volume ratio can result in strong antibacterial/fungal activity that enables CuONPs to interact with microbial membranes and release their metallic ions in solution (Ramyadevi et al., 2012). Gabbay et al. (2006) showed that different fabric types treated with different concentrations of CuONPs resulted in 99.5 to $99.9 \%$ reductions in the numbers of several pathogens. For example, Polyester treated with 10\% CuONPs reduced Escherichia coli (gram-negative bacteria) and HIV-1 (virus) by more than $99.9 \%$ after an exposure time of $1 \mathrm{~h}$ and $20 \mathrm{~min}$, respectively (Gabbay et al., 2006). The worldwide production of copper oxide nanoparticles was estimated to be 200 ton/year in 2010 that results in $>37$ ton/year 
emission to wastewater and $>36$ ton/year emission to soil (Keller et al., 2013). Most of the CuONPs produced are used in applications in medicine, cosmetics, antibacterial activities, gas sensors, catalysts, electronics, optics, coatings, pigments and paints (Chowdhuri et al., 2004; Gabbay et al., 2006; Battez et al., 2010; US Research Nanomaterials, 2017). Part of CuONPs from these applications are eventually released to the wastewater stream through usage and inappropriate disposal (Keller et al., 2013).

\subsection{Public health and environmental impact of AgNPs and CuONPs}

Nanoparticle toxicity has been the focus of many studies. AgNPs are known for their antibacterial activity on even antibiotic resistant-bacteria (Lansdown, 2004), and their potential toxic effects have been observed in various aquatic and terrestrial organisms, including plants, algae, fungi, fish, earthworms and bacteria (Colman et al., 2013; Cunningham et al., 2013; Panacek et al., 2009; Shoults-Wilson et al., 2011; Masrahi et al., 2014). In addition, the toxicity of CuONPs has been reported in plants, algae, mussel, fish, and bacteria (Anjum et al., 2015; Aruoja et al., 2009; Hu et al., 2014; Song et al., 2015; Baek \& AnYoun-Joo, 2011).

Furthermore, AgNPs have been found to cause toxic effects in human cells (Kawata et al., 2009; Asharani et al., 2010; Liu et al., 2011; Juling et al., 2017), and the capability of CuONPs to have harmful effects on humans and the environment has been shown in many studies (Li et al., 2007; Yang et al., 2010; Rotoli et al., 2012; Jang et al., 2016). However, these studies were done with human cells and other organisms from pure cultures and exposed directly to the nanoparticles; thus, the findings may not necessarily represent reallife situations. For example, several studies have shown that AgNPs and CuONPs toxicity 
is a dose-dependent matter (Li et al., 2007; Siddiqui et al., 2013; Jang et al., 2016; Juling et al., 2017). Even though these nanoparticles are used in a diverse range of consumer goods, there is minor exposure to the broader population, since the amounts released are relatively small. Thus, the main concern is likely the toxicity to humans who are in direct and continuous contact with nanoparticles, such as workers in the nanotechnology industry or patients exposed through medical material and devices. However, exposure to low concentrations must be considered as the expanding use of AgNPs in these products can cause many problems due to increased accumulation after repeated exposures over long periods, and the potential risk of bacterial resistance. Consumers are exposed to nanoparticles in a variety of ways. Although it is generally agreed that absorption of nanoparticles through human skin is minimal (Wijnhoven et al., 2009), there are explicit indications of nanoparticle deposition through inhalation by exposure and vulnerability to spray and aerosol products and cosmetic powders (Lorenz et al., 2011). Regardless, the unintentional uptake must be considered, and the use of consumer products and medical devices (Asharani et al., 2010) are believed to be the main cause of human exposure to AgNPs. A study done by Johnston et al. (2010) showed that AgNPs could be taken up by the barriers of the gastrointestinal tract (GIT), skin, and lungs, enter the bloodstream and be distributed throughout the body. However, as several studies have shown, uptake by tissues is size-dependent (Lankveld et al., 2010), and it is unclear whether AgNPs uptake, distribution and accumulation are caused by silver nanoparticles or silver ions. In addition, the interaction and transformation of AgNPs in the environment before reaching the cell should be considered when studying their impacts. 


\subsubsection{Toxicity mechanisms}

There are several reasons for the toxicity of AgNPs and CuONPs, including the dissolution and release of metal ions (Feng et al., 2000; Xiu et al., 2012; Jo et al., 2012; Song et al., 2015 ) and their very small size which enables them to easily penetrate the cell wall. Once inside a cell, there is a possibility of interaction with the cell constituents. Several studies identified the potential mechanisms of AgNPs and CuONPs toxicity:

\section{Dissolution of AgNPs and CuONPs}

Dissolution and release of nanoparticles ions that interact with biomolecules within the cell membrane (e.g., sulfur-containing proteins) can lead to further damage and cell death (Lansdown, 2004; Bondarenko et al., 2012; Wang et al., 2013; Adam et al., 2015).

\section{Attachment to the cell surface}

The very large surface area to volume ratio allows AgNPs and CuONPs to make strong connections with a microorganism's surface, enabling them to accumulate and alter the membrane structure (Lansdown, 2004). AgNPs can inhibit the respiration process of a bacterial cell by binding to the cell wall and membrane (Klasen, 2000).

\section{Penetration into the cell}

Some nanoparticles such as AgNPs get attached to the cell membrane and can easily penetrate to the inside of the cell. This is likely due to the interaction of AgNPs with sulfurcontaining proteins in the cell membrane. Also, AgNPs interact with the protein inside the cell and the phosphorus-containing compounds, such as DNA, causing further damage and cell death (Rai et al., 2009). Asharani et al. (2010) investigated the impact of AgNPs on red human blood cells and observed various types of cytotoxicity, including significant cell 
lysis, cell membrane damage and changes in cell morphology, after they were exposed to $100 \mu \mathrm{g} / \mathrm{mL}$ of AgNPs for one hour. There were no toxic effects when the cells were exposed to silver ions under the same conditions. This indicated that toxicity was only due to the AgNPs damaging the DNA, as was explained by the authors.

Silver and copper ions also induce redox reactions, which can form reactive oxygen species (ROS) due to the interaction of metal ions with oxygen, causing oxidative stress, DNA damage and cell death (Jomova \& Valko, 2011; Xu et al., 2012). As well, Choi \& Hu (2008) observed that the inhibition of nitrifying bacteria by AgNPs correlated with intercellular ROS concentration. One of the ROS that copper ions can create when being in contact with cell membrane are hydroxyl free radicals, which are very toxic to the lipid membranes of cells and can destroy the membrane structures through oxidation. Subsequently, the intracellular materials (such as $\mathrm{K}^{+}$, potassium ions) seeps out of the cells through the damaged membrane until they can no longer sustain fundamental biochemical processes and succumb to death and cell lysis (Wei et al., 2010). Copper ions $\left(\mathrm{Cu}^{2+}\right)$ interact with the cell components and, according to Fenton reaction, are reduced to $\mathrm{Cu}^{+}$, which is responsible for cell deactivation (Meghana et al., 2012). Another study by Ganesh et al. (2010) showed that adding copper ions can cause the respiration rate to decrease by $55 \%$ when applying both coliform and ammonia-oxidizing bacteria in activated sludge, while no significant inhibition was observed when the same amount of copper oxide nanoparticles was added.

Despite the findings of many studies regarding the toxicity of AgNPs and CuONPs on cells of different types of organisms, proper analysis and prediction need to be conducted according to many factors, which make it very difficult to consider all the impacts. For 
example, Song et al. (2015) observed that aggregation of CuONPs helps them remain in nanostructured form, that is, highly reactive and toxic. As well, manufacturing methods can affect the antibacterial properties of AgNPs. Samberg et al. (2011) observed different antibacterial performances of AgNPs due to how the AgNPs suspension is prepared. They found that synthesizing AgNPs after base reduction, coating them with formaldehyde and washing them in a phosphate buffer made them much less toxic to all the studied bacteria strains, compared to unwashed AgNPs. It is believed that washing decreases the formaldehyde content.

\subsection{Transformation of AgNPs and CuONPs in the environment}

The high surface to volume ratio of nanoparticles makes them highly dynamic and active in environmental systems. How the nanoparticles transform can affect their state, transport, and toxic properties. For example, AgNPs may become sulfidized in the environment, which can change their aggregation state, surface chemistry charge, as well as their ability to release toxic silver ion (Levard et al., 2011), therefore, affecting their behavior and toxicity. The most common AgNPs and CuONPs transformation mechanisms are:

\subsubsection{Chemical transformation}

Oxidation and reduction are the main chemical transformations that AgNPs and CuONPs may undergo in both aquatic and terrestrial environments. Releasing silver ion is accomplished by oxidation of $\operatorname{Ag}(0)$ to $\operatorname{Ag}(\mathrm{I})$. Oxidation usually happens in water and aerated soils, while reduction occurs in oxygen-deficient environments such as groundwater and carbon-rich sediments. In addition, nanoparticles' behavior can change under redox conditions. Sunlight exposure is an example that causes the chemical 
transformation of nanoparticles and can affect their coatings, oxidation state, and generation of ROS (Lowry et al., 2012). Cheng et al. (2011) observed that the degradation of gum Arabic coating used on AgNPs resulted in aggregation and precipitation. AgNPs and CuONPs are believed to have a strong affinity to react with inorganic and organic sulfide compounds in water, soil, and air before being in contact with cells, which reduces their toxicity. Moreover, the presence of organic matter in the environment changes the surface chemistry of both AgNPs and CuONPs affecting their stability, dissolution, and aggregation.

\subsubsection{Physical transformation}

Several factors can induce the aggregation of nanoparticles with themselves or with other particles in the surrounding environment. Aggregation reduces the effectiveness of the very large surface area to volume ratio that in turn affects the presence of the nanoparticles. The concentration and size distribution of the nanoparticles can change, and thus affect their properties, including stability, transportation, settling and uptake by organisms and toxicity. In addition, it can decrease the available surface area, and hence reduce the reactivity of the nanoparticles (Hotze et al., 2010). As a result, the toxicity decreases, especially if the surface area is the main parameter that induces reaction such as dissolution or formation of ROS (Lowry et al., 2012). On the other hand, aggregation may stimulate the persistence of the nanoparticles by preventing dissolution and degradation.

\subsection{Parameters affecting the behavior and impact of nanoparticles}

The chemical complexity of AgNPs, CuONPs and suspensions makes it difficult to analyze their behavior (Adams \& Kramer, 1999; Liu \& Hurt, 2010; Chappell et al., 2011). The 
stability and dissolution of engineered nanoparticles (ENPs) depend on many factors related to both the characteristics of nanoparticles and the surrounding environment. Even though AgNPs and CuONPs are known for their antibacterial activities, accurate analyses and correlations are not always obtained since most studies consider the characteristics of nanoparticles suspended in nanopure water when investigating their environmental and health impacts. This can cause significant errors because the characteristics of nanoparticles in different environments can vary greatly. Kittler et al. (2010) and Lee et al. (2012) showed that AgNPs behavior in deionized water is very different from when they are suspended in biological media. Thus, it is critical to have sufficient information about nanoparticles under particular exposure conditions to correlate any observed effects to their properties and dosage.

For example, the transformation of AgNPs from one chemical form to another strongly impacts silver toxicity (Reidy et al., 2013 ). In addition, the characteristics of nanoparticles suspended in water are usually different than their characteristics in other solutions, such as buffer, seawater, microorganism culture media and wastewater. Thus, it is crucial to characterize the nanoparticles in the solution of interest, to account for all types of impact.

AgNPs and CuONPs characteristics, such as the coating and surface nanoparticle charge, can also change their behavior and impact. Various coatings are used to stabilize the AgNPs against agglomeration (Chappell et al., 2011), dissolution of nanoparticles (Yang et al., 2011) and to modify their biological activity (Yang et al., 2011; Austin et al., 2011; Chappell et al., 2011). These studies showed that the AgNPs toxicity levels may change when using different types of coatings, and this could also cause variable surface charges (Suresh et al., 2012), which in turn affect the behavior and interaction of AgNPs with the 
surrounding environment. The surface charge was also found to be a factor affecting AgNPs toxicity (El Badawy et al., 2010).

Nanoparticle size and shape are important factors when studying AgNPs and CuONPs, and several studies reported that nanoparticle size is one of the main factors that influence AgNPs and CuONPs toxicity (Johnston et al., 2010; Pal et al., 2007; Powers et al., 2011). Generally, smaller nanoparticles are proven to be more toxic (Morones et al., 2005). For example, Choi \& $\mathrm{Hu}$ (2008) found a relationship between AgNPs size and the concentration of the intracellular ROS and observed higher ROS concentration when using $\leq 5 \mathrm{~nm}$ AgNPs. However, nanoparticles are of various sizes and shapes in the environment, and it is important to consider all the impacts when studying their interaction and toxicity. The fact that CuONPs toxicity depends on size is based on several studies, including Kumaran et al. (2014) who showed that $14 \mathrm{~nm} \mathrm{CuONPs}$ at $100 \mu \mathrm{g} / \mathrm{mL}$ for $24 \mathrm{~h}$ did not have significant toxic impact on a human breast cancer cell compared to the control, even though ROS gene expression was higher in these cells. Another study by Siddiqui et al. (2013) demonstrated that exposure of human hepato carcinoma cells to $22 \mathrm{~nm}$ CuONPs, at a concentration range of 2 to $50 \mu \mathrm{g} / \mathrm{mL}$ for $24 \mathrm{~h}$, caused toxicity due to the generation of ROS. Another study by Pal et al. (2007) demonstrated that the interaction of AgNPs with E. coli depended on the AgNPs shape. They showed that the truncated triangular AgNPs with a $\{111\}$ lattice plane as the basal plane, displayed the strongest biocidal action when compared to spherical and rod-shaped silver nanoparticles.

Several properties of the surrounding environment can affect the behavior of AgNPs and CuONPs, including the ionic strength (Lee et al., 2012; Yang et al., 2011; Liu et al., 2010), pH (Adeleye et al., 2014; Qu et al., 2010), extracellular polymeric substances (Adeleye et 
al., 2014), dissolved oxygen concentration, and organic matter concentration. A study by Yang et al. (2011) found that higher toxicity in all types of AgNPs can occur when a low ionic strength medium is presented. In addition, Liu \& Hurt (2010) investigated silver ion release kinetics and found that the release of silver ions increased from 0 to $37^{\circ} \mathrm{C}$ and decreased at high $\mathrm{pH}$ levels or in the presence of humic or fulvic acid. They found that dissolved oxygen is critical in AgNPs dissolution, even at low $\mathrm{pH}$ levels. The dissolution rate of AgNPs can be decreased by oxygen striping, high $\mathrm{pH}$ levels, high concentrations of organic matter (i.e. humic or fulvic acids), different coatings such as stabilizing citrate and temperature reduction (Liu \& Hurt, 2010). In addition, Qu et al. (2010) found that the antibacterial activity of AgNPs decreased at acidic $\mathrm{pH}$ levels $\leq 2$ and increased at temperatures of $37^{\circ} \mathrm{C}$ or higher, due to the changes in the colloidal properties of AgNPs that causes them to aggregate and become less toxic. Adeleye et al. (2014) showed the combined effect of $\mathrm{pH}$, ionic strength and extracellular polymeric substances on CuONPs stability and dissolution.

High organic matter concentrations can stabilize AgNPs and CuONPs in any biological medium, including water, wastewater, sludge, and soil. However, the effects of organic matter can be modified by several factors, including the presence of polymer. Chappell et al. (2011) demonstrated that through organic matter such as humus can stabilize AgNPs, high levels of the polymer can increase AgNPs dissolution and release silver ions. In addition, a study by Khan et al. (2011) found that the measured zeta potential was close to zero, and the AgNPs were unstable and agglomerating. In the presence of extracellular polymeric substances (EPS), the agglomeration was reduced, and the zeta potential was $30 \mathrm{mV}$. Khan et al. (2011) also found that adsorption of EPS on AgNPs depends on the 
$\mathrm{pH}$, salt and ESP concentrations, and showed that $15 \%$ of the silver was released after dispersion in a growth medium contained ESP. Thus, the presence of EPS at high concentrations can increase the stability of AgNPs and reduce agglomeration.

\subsection{Mass flow of silver and copper into wastewater, sludge, and soil}

The emission of nanoparticles into the environment can occur throughout the entire life cycle of products containing nanoparticles, including during the waste phase (Wiesner \& Plata, 2012). AgNPs can be released from different materials, including textiles (Benn \& Westerhoff, 2008; Geranio et al., 2009) and paint (Kaegi et al., 2010). Keller et al. (2013) found that paints and pigments, coatings and cosmetics make up $42 \%$ of the nanoparticles released into the environment. These three industries are responsible for 82 to $87 \%$ and 89 to $97 \%$ of the total discharged into soil and water, respectively (Keller et al., 2013). Kaegi et al. (2010) showed that over $30 \%\left(0.5 \mathrm{mg} / \mathrm{m}^{2}\right)$ of the original mass of AgNPs in the paint could be discharged into the atmosphere after only one year and of this $80 \%$ was discharged during the first eight runoff events. The discharged silver particles were generally less than $15 \mathrm{~nm}$ in size, and most of this silver was changed into less lethal forms, such as $\mathrm{Ag}_{2} \mathrm{~S}$ (Kaegi et al., 2010).

Wastewater is considered one of the main pathways for many types of nanoparticles to reach soil and water systems, including AgNPs and CuONPs. Keller et al. (2013) showed that of the total nanoparticles passed through wastewater treatment plants, approximately 3 to $25 \%$ would end up in water bodies via the wastewater effluent, and 44 to $47 \%$ would be released into the soil via biosolids. Textiles are the primary sources for AgNPs and CuONPs in the water system (Blaser et al., 2008), and studies confirmed the release of 
AgNPs from the fabric after several washing cycles (Benn \& Westerhoff, 2008; Geranio et al., 2009). Geranio et al. (2009) also found that just one wash released from 1 to $45 \%$ of silver depending on the type of fabric and the manufacturing method. Since textiles and other products with AgNPs or CuONPs are increasingly used, the release of silver and copper into wastewater systems is a serious concern and growing issue. In wastewater treatment plants, although most nanoparticles will be removed from the liquid stream and concentrated in sludge, a significant amount will escape in the wastewater effluent. For example, $\mathrm{Li}$ et al. (2016) reported that although $96.4 \%$ of AgNPs are removed from wastewater during treatment, the AgNPs discharged with the wastewater effluent can reach $33 \mathrm{~kg} /$ year in Germany. Also, a study of mass balance by Kaegi et al. (2011) showed that of the initial added AgNPs, $5 \%$ (7.2 g) was released from wastewater treatment plants with the effluent, approximately $85 \%(110 \mathrm{~g})$ was in sludge and 5\% (7.8 g) remained in the treatment plant at the end of 43 days. They stated that due to the physical and chemical transformations of silver nanoparticles, only after the fourth day of the testing, the amounts in sludge, effluent and the treatment plant were $90 \%, 2.5 \%$ and $7 \%$, respectively (Kaegi et al., 2011).

The concentration of AgNPs or CuONPs in sludge raises concerns about their subsequent activity and impact on soil ecosystems after land application. While soil amendment with sludge is the main way AgNPs enter the terrestrial environment, the total silver and copper concentrations in sludge are highly variable. According to a survey conducted by the Environment Protection Agency (EPA), 74 wastewater treatment facilities had concentrations in the range of 1.94 to $856 \mathrm{mg} / \mathrm{kg}$ sludge for silver and 115 to $2580 \mathrm{mg} / \mathrm{kg}$ sludge for copper (EPA, 2009). While the EPA regulates the application of sewage sludge 
to agricultural lands based on the annual loading rate of specific heavy metals, such as arsenic, cadmium, mercury, and copper, it does not regulate the silver applied to agricultural lands. According to Ontario regulations, copper concentration should not exceed $1700 \mathrm{mg} / \mathrm{kg}$ TS of sludge applied to soil and the maximum annual loading rate should not exceed 760 ton/ha/5 years (CCME, 2010; OMAFRA, 2019). Also, according to US EPA, maximum copper concentration in sludge and annual loading rate to soil should not exceed $1500 \mathrm{mg} / \mathrm{kg}$ TS of sludge and $75 \mathrm{~kg} / \mathrm{ha} /$ year, respectively (EPA, 2018).

\subsection{Behavior and transformation of nanoparticle during wastewater and sludge treatment}

After the release of AgNPs or CuONPs into the environment, structural and surface chemistry changes may be observed, and there are many potential transformations, including stability, dissolution, and agglomeration. Understanding the dynamics of the exposure concentration, exposure time (short or long term) and the changes in physicochemical properties is essential to determine the behavior and ultimate status of these nanoparticles.

The changes in the physicochemical properties of AgNPs start in the sewer system, particularly when sulfide concentrations are high (Nielsen et al., 2006), and AgNPs can be retained and accumulate in biofilms in the sewer system (Fabrega et al., 2009). Thus, the biofilms in sewer systems can be temporary storage basins for AgNPs during dry seasons and a source of AgNPs during rainy seasons, which can cause elevated levels of AgNPs in both wastewater and sludge. In a wastewater treatment plant, AgNPs go through physical,

chemical, and biological treatment processes designed to remove organic matter and 
nutrients. AgNPs can be removed from the aqueous phase by mechanisms such as aggregation, precipitation, settling, and interaction with biomass (biosorption), which is important in the removal of AgNPs (Parka et al., 2013). Although the dissolution of AgNPs has been observed, association with biosolids is reportedly the primary removal mechanism from wastewater (Shafer et al., 1998; Kaegi et al., 2011). More than 94\% of AgNPs entering a wastewater treatment plant are associated with sludge solids (Shafer et al., 1998), and more than $85 \%$ will end up in sludge (Kaegi et al., 2011). In addition to their association with biomass and solids, AgNPs are also transformed into different species, such as ionic silver $\left(\mathrm{Ag}^{+}\right)$and silver oxide $\left(\mathrm{Ag}_{2} \mathrm{O}\right)$ (Shafer et al., 1998; Kaegi et al., 2011). Sulfidation of AgNPs can occur in the presence of sulfur compounds in the aqueous phase, in both aerobic and anaerobic conditions. The interaction of AgNPs with sulfur, and the subsequent production of silver sulfide results in a significant reduction in the toxicity of AgNPs due to the lower solubility of silver sulfide, and limits short-term environmental impacts (Levard et al., 2012). AgNPs interact strongly with sulfur to produce silver sulfide $\left(\mathrm{Ag}_{2} \mathrm{~S}\right)$, both when they are discharged into the sewer system and during wastewater treatment (Kim et al., 2010; Kaegi et al., 2013).

Lorenz et al. (2012) reported that AgNPs could transform into silver chloride (AgCl) due to the bleach used for washing textiles. The interaction of AgNPs with $\mathrm{Cl}^{-}$can depend on the $\mathrm{Ag} / \mathrm{Cl}$ ratio in the solution and with low $\mathrm{Ag} / \mathrm{Cl}$ ratios increase dissolution ( $\mathrm{Li}$ et al., 2010). Small amounts of $\mathrm{Cl}^{-}$interact with $\mathrm{Ag}^{+}$to form $\mathrm{AgCl}$, which precipitates and reduces the toxicity effect of the $\mathrm{Ag}^{+}$. High levels of $\mathrm{Cl}^{-}$in the solution can form bioavailable ionic compounds, such as $\mathrm{AgCl}^{2-}, \mathrm{AgCl}_{3}{ }^{2-}$, and $\mathrm{AgCl}_{4}{ }^{3-}$, and increase the toxicity for organisms. 
CuONPs also undergo various transformations once they are in wastewater, including aggregation, dissolution, and settling. Conway et al. (2015) showed that the presence of $\mathrm{Cl}^{-}$ , $\mathrm{SO}_{4}{ }^{2-}, \mathrm{PO}_{4}{ }^{3-}$ and $\mathrm{HCO}_{3}{ }^{-}$in water could cause these ions to react with free copper, and thereby form copper complexes that lead to the precipitation of ionic copper. As well, the presence of natural organic matter can change the surface charge of CuONPs and affect their agglomeration behavior. However, the impact of organic matter is governed by the presence of other ions such as $\mathrm{Ca}^{2+}$, that can be rapidly adsorbed onto the surface of the organic matter and reduce their activity on CuONPs (Son et al., 2015). Generally, CuONPs and AgNPs are removed from wastewater through primary and secondary treatments, and are concentrated in sludge (Kaegi et al., 2011).

\subsection{Potential applications of nanomaterials in wastewater treatment}

Different applications of nanoparticles in wastewater treatment have been discussed in several studies. For examples, Suopajärvi et al. (2013) showed that dicarboxylic acid nanocellulose was able to flocculate wastewater very efficiently compared to commercial polymer, which resulted in lower residual turbidity and COD in the settled suspension. Another study by Lakshmanan and Rajarao (2014) showed a 95\% reduction of sludge water content in less than 5 min when using magnetic nanoparticles as flocculants for sedimentation of sludge, while only $30 \%$ reduction was observed after 60 min when using either protein or chemical coagulant. Also, AgNPs have been incorporated in the antifouling membranes to prevent attachment of bacteria and the formation of biofilm due to their high antimicrobial activities (Qu et al., 2013). Metal-based nanomaterials have been tested for removing a variety of heavy metals such as arsenic, lead, mercury, copper, 
cadmium, chromium, nickel and have shown a great potential to outcompete activated carbon (Sharma et al., 2009).

\subsection{Sludge treatment}

Sludge treatment includes different types of crucial processes utilized to stabilize and dewater sludge. Some of the main sludge treatment processes are anaerobic digestion, aerobic digestion, chemical stabilization, heat stabilization, conditioning, dewatering, and incineration. The stabilization and dewatering processes qualify the sludge for land application, and at this stage, it is called biosolids (EPA, 2009; CCME, 2010).

\subsubsection{Anaerobic digestion of sludge}

Anaerobic digestion is the most commonly used sludge stabilization method at wastewater treatment plants, and it involves using microorganisms to convert organic matter into carbon dioxide and methane in the absence of oxygen. There are two types of anaerobic digestion: mesophilic, in which the system operates at moderate temperatures of 30 to $38^{\circ} \mathrm{C}$, and thermophilic, where the system operates at high temperatures of 50 to $57^{\circ} \mathrm{C}$. The majority of current anaerobic digestion systems are mesophilic, though they can also be used in two stages such as mesophilic-thermophilic digestion.

Anaerobic digestion of sludge is comprised of four main stages: hydrolysis, fermentation, acetogenesis, and methanogenesis that activate a series of physical, chemical, and biochemical reactions (Metcalf \& Eddy, 2003). Several operational and environmental factors need to be considered for the process to be successful, including solid and hydraulic retention times, temperature, $\mathrm{pH}$, the concentration of inhibitory substances, nutrients and 
trace elements (Metcalf \& Eddy, 2003). When studying AgNPs and CuONPs during anaerobic digestion, it is also important to consider the physical, chemical, and biochemical reactions, as well as the operational parameters. Relatively few studies have investigated the behavior and impact of metal nanoparticles during anaerobic digestion of sludge. OteroGonzález et al. (2014) studied the effect of low concentrations of CuONPs $(1.4 \mathrm{mg} \mathrm{Cu} / \mathrm{L})$ on high-rate anaerobic digestion and found that most CuONPs were associated with sludge solids and 20 to $32 \%$ escaped in the effluent. They also reported that CuONPs inhibited the anaerobic digestion process.

\subsubsection{Sludge conditioning}

Chemical conditioning is used to improve the solid-liquid separation and prepare sludge for dewatering. It involves using chemicals to help destabilize the colloidal particles, which results in agglomeration of small particles into larger settable flocs. Some of the chemicals used are inorganic coagulants such as alum, sulfuric acid, ferrous sulfate and ferric chloride (Metcalf \& Eddy, 2003), as well as organic (synthetic) polymers which are most frequently used. Chemical conditioning works by coagulation of solid particles and release of mainly free water, and it can help to reduce the initial sludge moisture content from $90-99 \%$ to 65 $85 \%$, depending on the type of sludge and dewatering device used (Turovskiy \& Mathai, 2006). Adding chemicals either lowers or raises the sludge $\mathrm{pH}$ to the point that small particles coagulate into larger ones that can easily be separated from the water. In addition, chemical conditioners can change the surface charge and chemistry of the particles, causing them to destabilize. The amount and kind of conditioning chemicals used depend on the sludge type, which can be primary, secondary, and treated sludge, daily changes in incoming wastewater characteristics, and operational conditions at the treatment plant. 
It is important to consider sludge conditioning when studying the effect of nanoparticles. Many studies have shown that a large percentage of AgNPs entering a wastewater treatment plant will be associated with sludge and biosolids. Studying the effect of chemical conditioning is crucial, as it changes the surface chemistry of the nanoparticles and can have a major impact on their behavior. There have been very few literature studies that regard the behavior and impact of nanoparticles during and after chemical coagulation/flocculation processes. Studies mainly investigated the fate of AgNPs during sludge anaerobic digestion and post processing, such as composting and incineration (Lombi et al., 2013; Ma et al. ,2013; Impellitteri et al., 2013). The impact of the conditioning process has not yet been investigated.

\subsubsection{Ferric chloride and alum}

Sludge conditioning using ferric chloride $\left(\mathrm{FeCl}_{3}\right)$ and aluminum sulfate $\left(\mathrm{Al}_{2}\left(\mathrm{SO}_{4}\right)_{3} \bullet(14-\right.$ 18) $\mathrm{H}_{2} \mathrm{O}$ ) is achieved through a series of hydrolysis, adsorption, and precipitation reactions. They work through surface charge neutralization of particles; ferric chloride dissociates into ferric and chloride ions, and aluminum sulfate dissociates into aluminum and sulfate ions. Ferric and aluminum ions react with water to produce ferric and aluminum hydroxide species, and if they are deposited on the surfaces of sludge particles, they can lead to charge neutralization (Dentel, 2001). This is due to the positive or neutral charge that ferric and aluminum hydroxide have at $\mathrm{pH}$ values below 8 . Dentel (2001) also reported that these types of adsorption and precipitation reactions occur in a patchwork manner on particle surfaces. 


\subsubsection{Synthetic polymers}

Polymers (organic polyelectrolytes) have many advantages over inorganic metal coagulants, including ease of handling, a smaller feed system, and the quantities used are many times less than those with inorganic coagulants. Polymers are long chains of repeated large organic molecules, and they are typically classified based on their charge to anionic, non-ionic and cationic. Polyelectrolytes function according to their surface charge density, molecular weight, and two main conditioning mechanisms: the bridge model and the patchwise model. The bridge model involves adsorption of polyelectrolyte segments on particle surfaces, which then get tangled and hold the particles together. The patchwise model also involves adsorption of polyelectrolyte segments on particle surfaces, which then create negative and positive charge zones that pull the particles together by electrostatic attraction through the alignment of the negatively and positively charged areas. Bratby (2006) stated that the adsorption mechanisms depend on two important factors: the chemical characteristics of the polyelectrolytes and the adsorbent surface. It has been suggested that some of the causes of adsorption could be cation exchange (cationic polyelectrolyte adsorption on clay particles), electrostatic linkages, hydrogen bonding, and ionic bonding (adsorption of anionic polyelectrolyte to clay particles). Using polyelectrolytes during sludge conditioning can also affect the surface charge and other characteristics of nanoparticles in sludge, and thereby cause changes in the behavior and fate of nanoparticles. Some polymers are used for surface coating of nanoparticles to make them more resistant to aggregation. However, the polymers used for surface coating are not the same as the water-soluble polymers applied for water and wastewater treatment. A study by Kvitek et al. (2008) investigated the stabilization capability of various polymers 
and surfactants on AgNPs and found that treatment of AgNPs with polymer polyvinylpyrrolidone (PVP) 360 made them more resistant to aggregation. This was due to the strong bonding of PVP 360 to AgNPs by a nitrogen atom in the polymer molecular. Therefore, differences in the molecular composition of polymers and the availability of functional groups in the environment can affect the reactions and stability of nanoparticles. CuONPs were also found to be significantly affected by the presence of EPS, which include biopolymers produced by bacteria in environments such as water, wastewater and soil samples (Miao et al., 2015). Effect of synthetic polymers on CuONPs during wastewater treatment has not been studied previously.

\subsubsection{Sludge lime stabilization}

Lime stabilization is accomplished through the addition of lime until reaching a $\mathrm{pH}$ of 12 or higher, and it is maintained for two hours (Metcalf \& Eddy, 2003). Lime works by raising the sludge $\mathrm{pH}$ level and increasing its porosity. This is to ensure the deactivation of pathogens and other microorganisms and prevent the production of odorous gases by combining high $\mathrm{pH}$ and high temperature. Lime can also cause the conversion of hydrogen sulfide to sulfide and bisulfate ions that are not volatile at elevated $\mathrm{pH}$ levels and hence reduces the sludge odor (Turovskiy \& Mathai, 2006). In addition, the increase in pH level affects the solubility of minerals, volatilization of ammonia, and precipitation of phosphorus. Sludge lime treatment commonly results in increasing the volume of wastewater sludge and thus increase its disposal cost. In addition to chemical stabilization, lime may be used for sludge conditioning after ferric and alum treatment (Turovskiy \& Mathai, 2006). There are two main lime products used in the sludge conditioning/stabilization process: calcium oxide, known as quicklime $(\mathrm{CaO})$ and calcium 
hydroxide, known as slacked or hydrated lime $\left(\mathrm{Ca}(\mathrm{OH})_{2}\right)$. Calcium oxide is usually used for dewatered sludge, while calcium hydroxide is used for liquid sludge. Generally, calcium oxide is preferred, because it is added as a powder, and this can effectively dewater the sludge as the water interacts strongly with calcium. Also, the elevated $\mathrm{pH}$ level and heat act strongly to deactivate pathogens (Vesilind et al., 1986). There are very few studies in the literature that investigated the behavior of nanoparticles during the lime treatment of sludge. One study carried out by Ma et al. (2013) showed that $\mathrm{Ag}_{2} \mathrm{~S}$ is the dominant silver compound found after lime/heat treatment of sludge.

\subsection{Land application of sludge}

\subsubsection{Impact of nanoparticles after land application of sludge}

Large volumes of biosolids are produced daily from wastewater treatment plants, and they are typically land applied. For example, approximately 390 million liters per day of wastewater processed in the city of Ottawa produces about 39 dry tonnes per day of biosolids that are used as soil fertilizer on agricultural lands (City of Ottawa, 2014). However, the application of biosolids on land may also provide a pathway for various contaminants, including AgNPs and CuONPs, to enter the soil. In the regulations for biosolids land application, silver is commonly considered a secondary contaminant. It is usually referred to as total silver without distinguishing dissolved silver $\left(\mathrm{Ag}^{+}\right)$and particulate silver such as AgNPs (EPA, 2018). There are currently no limits on nanoparticle concentrations in sludge in North America.

Studies have shown that AgNPs and CuONPs can affect soil microbial activity, and this is a concern because soil organisms are responsible for organic matter decomposition and 
nutrient cycling. Physicochemical properties of soil are important to consider when evaluating the toxicity of nanoparticles to soil microorganisms (Shoults-Wilson et al., 2011). For example, high levels of organic matter and humic and fulvic acids can reduce the toxic effect of AgNPs and CuONPs (Dinesh et al., 2012). In addition, when assessing the potential risk of nanoparticles, it is important to consider both the exposure time and the type of effect. The risk assessment must also consider the toxicity of transformed nanoparticles, not just those that are pristine or freshly made. AgNPs and CuONPs are not stable under most environmental conditions and will likely transform once introduced into an ecosystem (Chen et al., 2012). For example, AgNPs are highly likely to react with sulfur, chloride, and organic matter (Kramer et al., 2002). The reaction of AgNPs with other chemicals can also alter their toxicity, such as the case with sulfur where relatively insoluble $\mathrm{Ag}_{2} \mathrm{~S}$ is formed with less toxicity (Levard et al., 2012). Recent studies suggest that there are significant concentrations of $\mathrm{Ag}_{2} \mathrm{~S}$ in most types of sludges (Kaegi et al., 2013; Kim et al., 2010).

\subsubsection{Effect of AgNPs and CuONPs on soil microorganisms}

Soil microorganisms interact strongly with a wide range of biota in terrestrial ecosystems. Plants, for example, are colonized by many microorganisms that live in different parts. Rhizosphere microorganisms provide many functions that benefit plants, such as nutrient uptake, protection against pathogens, and enhanced abiotic stress tolerance, and they also promote plant development (Berendsen et al., 2012). However, some rhizosphere microorganisms can cause disease and impede plant growth. Thus, soil microorganisms are critical to plant health, and it is very important to study the factors that affect them. Several studies investigated the role of AgNPs in soil, and their negative effect on microbial 
biomass, diversity, and plant health. For example, Kumar et al. (2011) showed the high toxicity of AgNPs in soil microbial communities, particularly Bradyrhizobium canariense bacteria, which is associated with plants. Another study by Colman et al. (2013) found that microbial biomass was $35 \%$ lower in mesocosms that were amended with $0.14 \mathrm{mg}$ AgNPs/kg soil compared to those with no AgNPs.

\subsubsection{Influence of soil organic matter on AgNPs and CuONPs}

Soil organic matter is one of the most important constituents of soil, as it is essential for plant growth. Soil organic matter contains approximately $56 \%$ of the organic carbon that is a source of energy and nutrients for soil microorganisms (Edwards et al., 1992). In addition, organic carbon such as humus is involved in the stability of aggregates and nutrients, and necessary for the water holding capacity of the soil. Some organic carbon compounds, such as polysaccharides, help hold mineral particles together as microflocs. The studies mentioned in the previous section highlight the toxic effects of AgNPs and CuONPs on soil microorganisms. Other nanoparticles, such as fullerene C60, were less toxic to soil respiration and microbial biomass, as demonstrated in a study done by Tong et al. (2007). They used two different concentrations: $1.0 \mu \mathrm{g}$ C60/g soil in aqueous suspension (nC60) and $1000 \mu \mathrm{g} \mathrm{C60/g} \mathrm{soil} \mathrm{in} \mathrm{granular} \mathrm{form,} \mathrm{and} \mathrm{incubated} \mathrm{the} \mathrm{soils} \mathrm{for}$ $180 \mathrm{~d}$. The soil was a silty clay loam with $4 \%$ organic matter and nearly neutral $\mathrm{pH}$ level (6.9). They found no significant inhibition in the activity of dehydrogenase (an enzyme that indicates oxidative microbial activity in soils), or in the activities of enzymes involved in nitrogen, phosphorus and carbon cycles in the soil. This suggests that C60 did not cause significant stress to soil microbial activity. They also linked the low C60 toxicity to the strong binding of C60 in soil organic matter and the reduced mobility of nanoparticles in 
the soil matrix due to adsorption of soil organic matter. Natural colloidal and soil organic matters can cause aggregation of AgNPs and CuONPs into larger particles and hence reduce their antimicrobial activity.

\subsubsection{Microorganisms selected for this study}

Soil is an ecosystem containing a wide variety of microorganisms that are important to maintaining life on earth. In Chapters four and five, the overall bacterial population was analyzed and mainly taking in consideration the phyla and genus levels. In Chapters six and seven, five different phyla were chosen for assessment: Acidobacteria, Actinobacteria, Bacteroidetes, Firmicutes and Proteobacteria based on their high concentration in sludge/soil mixture; together they make up $84 \%$ of the total phyla in soil (Siles, 2014). In addition to their concentration, they also perform different activities in the soil, as illustrated in Table 2-1. Fierer et al. (2007) showed a relationship between the availability of carbon and the relative abundance of the five selected phyla in the soil and found an increase in the relative abundance of $\beta$-Proteobacteria and Bacteroidetes with high sucrose amendment rates. They also identified a decrease in the relative abundance of Acidobacteria with high levels of sucrose amendment. The other bacterial phyla ( $\alpha$ Proteobacteria, Firmicutes, and Actinobacteria) did not show any significant change in the carbon levels in the soil.

Based on a $16 \mathrm{~S}$ rRNA gene analysis, Acidobacteria phylum is considered the most abundant and ubiquitous bacteria in soil (Janssen, 2006). However, these bacteria are rarely cultured, and consequently are a poorly studied phylum (Philip et al., 1998). Many Acidobacteria are considered acidophilic organisms, due to their ability to function in 
highly acidic environments and their efficient and effective mechanisms to release protons that ensure their intracellular environment remains at a neutral $\mathrm{pH}$. Acidobacteria is a metabolically and genetically diverse group compared to other phyla, such as Proteobacteria, based on their phylogenetic diversity and ecological distribution pattern (Hugenholtz et al., 1998). Acidobacteria is the most frequently detected phylum in molecular ecological surveys of soil types, which indicates their importance in soil systems (Hugenholtz et al., 1998).

Actinobacteria are the phylum of gram-positive bacteria. They are important and contribute to soil and plant health by decomposing dead organic matters, so it is readily available for plants. They perform much like fungi, and their colonies often grow extensive mycelia. In addition to decomposing organic matter, some soil Actinobacteria, such as Frankia, live symbiotically with plant roots and help with nitrogen-fixing (Servin et al., 2008).

Bacteroidetes, although found in the rhizosphere and bulk soil in relatively low concentrations, are highly diverse (Fierer et al., 2007). They are gram-negative bacteria with a wide range of genera, including anaerobic bacteria Bacteroides and aerobic bacteria Flavobacteria (Thomas et al., 2011). Some Bacteroidetes function in the digestive systems of humans and animals, and they can also be pathogenic (Thomas et al., 2011). The Bacteroidetes phylum is also known for its ability to degrade polymeric organic matter.

Firmicutes are less abundant in soil compared to other phyla, such as Proteobacteria and Acidobacteria (Janssen, 2006). Although most Firmicutes are gram-positive bacteria, some are gram-negative, and many produce endospores that are resistant to desiccation and can survive extreme conditions. Firmicutes make up approximately $1.8 \%$ of the total bacterial 
abundance in soil (Janssen, 2006). Their relative abundance may be underestimated due to their strong cell walls, and spores that make it difficult to lyse and release their DNA for quantification (Janssen, 2006). The common Firmicutes classes in the soil are Bacilli and Clostridia. Firmicutes are very important because some, such as the Bacillus polymyxa strain, are responsible for nitrogen fixation and the decomposition of cellulose (Górska et al., 2000). Proteobacteria are one of the main phyla of gram-negative bacteria, and they contain a wide range of pathogens, including Escherichia coli, Salmonella, Vibrio cholerae, and Helicobacter pylori (Madigan \& Martinko, 2005). Proteobacteria is the most dominant bacterial phylum in most soil types, though they are more abundant in rhizosphere soils than bulk soils (Fierer et al., 2007). The common classes of this phylum in soil are $\alpha$-Proteobacteria, $\beta$-Proteobacteria, $\gamma$-Proteobacteria and $\delta$-Proteobacteria, of which $\alpha$-Proteobacteria can thrive at very low nutrient levels. Overall, Proteobacteria include nitrogen-fixing bacteria that are very important for agriculture soil. For example, $\beta$-Proteobacteria is the dominant autotrophic ammonia oxidizers in soil.

Table 2-1 Studied phyla and their population and important action in the soil.

\begin{tabular}{|l|c|c|c|}
\hline \multicolumn{1}{|c|}{ Phylum } & $\begin{array}{c}\text { Relative } \\
\text { abundance in soil } \\
\text { (\%) }\end{array}$ & Action in soil & Reference \\
\hline Acidobacteria & $20 \%$ & Biological phosphorus uptake. & (Janssen, 2006) \\
\hline Actinobacteria & $13 \%$ & $\begin{array}{c}\text { Carbon cycling and nutrient } \\
\text { transformation in soils. } \\
\text { Phosphorus accumulation, } \\
\text { production of bioactive } \\
\text { compounds. }\end{array}$ & $\begin{array}{l}\text { (Janssen, 2006; } \\
\text { Kurtböke, 2012) }\end{array}$ \\
\hline Bacteroidetes & $5 \%$ & $\begin{array}{c}\text { Phosphorus accumulation, } \\
\text { degradation of complex organic } \\
\text { matter, i.e., proteins and } \\
\text { carbohydrates. }\end{array}$ & $\begin{array}{c}\text { (Thomas et al., 2011; } \\
\text { Janssen, 2006) }\end{array}$ \\
\hline Proteobacteria & $39 \%$ & Nitrogen fixation. & (Janssen, 2006) \\
\hline Firmicutes & $1.8 \%$ & $\begin{array}{c}\text { Nitrogen fixation and } \\
\text { degradation of cellulose. }\end{array}$ & $\begin{array}{c}\text { (Janssen, 2006; Górska } \\
\text { et al., 2000) }\end{array}$ \\
\hline
\end{tabular}




\subsection{Summary}

The antibacterial activity of AgNPs and CuONPs has attracted substantial interest from industries, and many used them in daily consumer products. This has resulted in their release into the environment, mainly through wastewater, which has become a matter of concern. Several studies have concluded that AgNPs and CuONPs have toxic effects on organisms due to their antibacterial activity. The interaction of AgNPs and CuONPs with their surrounding environments can influence their properties and affect their stability, aggregation, and dissolution, and change their toxicity. The physicochemical characteristics of nanoparticles can also influence their behavior, and size, surface area, shape, zeta potential, surface coating, agglomeration, and dissolution rate are particularly important for determining their biological interactions and impacts. In wastewater treatment plants, AgNPs and CuONPs are subjected to various chemical and biological processes which impact their physicochemical characteristics, and several studies have concluded that a significant portion of the nanoparticles accumulates in sludge solids, raising concerns about their effect and toxicity after land application of biosolids. Although several studies have examined the toxic effects of AgNPs and CuONPs on soil organisms, they used pristine nanoparticles in these studies. There is a knowledge gap in the literature on the fate, transformation and impact of AgNPs and CuONPs during biological and chemical wastewater and sludge treatment processes and after land application of biosolids using nanoparticles that already went through wastewater treatment under realistic conditions. 


\section{References}

Adam, N., Vakurov, A., Knapen, D., \& Blust, R. (2015). The chronic toxicity of CuO nanoparticles and copper salt to Daphnia magna. Journal of Hazardous Materials, 283, 416-422.

Adams, N. W., \& Kramer, J. R. (1999). Silver speciation in wastewater effluent, surface waters, and pore waters. Environmental Toxicology \& Chemistry , 18(12), 2667-2673.

Adeleye, A. S., Conway, J. R., Perez, T., Rutten, P., \& Keller, A. A. (2014). Influence of extracellular polymeric substances on the long-term fate, dissolution, and speciation of copper-based nanoparticles. Environmental Science \& Technology, 48(21 ), 12561-12568.

Anjum, N. A., Adam, V., Kizek, R., Duarte, A. C., Pereira, E., Iqbal, M., . . Ahmad, I. (2015). Nanoscale copper in the soil-plant system-toxicity and underlying potential mechanisms. Environmental Research, 138, 306-325.

Aruoja, V., Dubourguier, H.-C., Kasemets, K., \& Kahru, A. (2009). Toxicity of nanoparticles of $\mathrm{CuO}, \mathrm{ZnO}$ and $\mathrm{TiO} 2$ to microalgae Pseudokirchneriella subcapitata. Science of The Total Environment, 407(4), 1461-1468.

Asharani, P. V., Sethu, S., Vadukumpully, S., Zhong, S., Lim, C. T., Hande, M. P., \& Valiyaveettil, S. (2010). Investigations on the structural damage in human erythrocytes exposed to silver, gold, and platinum nanoparticles. Advanced Functional Materials, 20(8), 1233-1242.

Austin, L. A., Kang, B., Yen, C.-W., \& El-Sayed, M. A. (2011). Nuclear targeted silver nanospheres perturb the cancer cell cycle differently than those of nanogold. Bioconjugate Chemistry, 22(11), 2324-2331.

Babu, S., Claville, M. O., \& Ghebreyessus, K. (2015). Rapid synthesis of highly stable silver nanoparticles and its application for colourimetric sensing of cysteine. Journal of Experimental Nanoscience, 10(16), 1242-1255.

Baek, Y.-W., \& AnYoun-Joo. (2011). Microbial toxicity of metal oxide nanoparticles (CuO, NiO, $\mathrm{ZnO}$, and $\mathrm{Sb} 2 \mathrm{O} 3$ ) to Escherichia coli, Bacillus subtilis, and Streptococcus aureus. Science of The Total Environment, 409(8), 1603-1608. 
Battez, A. V., González, R., Blanco, D., Asedegbega, E., \& Osorio, A. (2010). Friction reduction properties of a $\mathrm{CuO}$ nanolubricant used as lubricant for a NiCrBSi coating. Wear, 268(12), 325-328.

Benn, T. M., \& Westerhoff, P. (2008). Nanoparticle silver released into water from commercially available sock fabrics. Environmental Science \& Technology, 42(11), 4133-4139.

Berendsen, R. L., Pieterse, C. M., \& Bakker, P. A. (2012). The rhizosphere microbiome and plant health. Trends in Plant Science, 17(8), 478-486.

Blaser, S. A., Martin, S., Matthew, M., \& Konrad, H. (2008). Estimation of cumulative aquatic exposure and risk due to silver: contribution of nano-functionalized plastics and textiles. Science of The Total Environment, 390(2), 396-409.

Bondarenko, O., Ivask, A., Käkinen, A., \& Kahru, A. (2012). Sub-toxic effects of CuO nanoparticles on bacteria: kinetics, role of $\mathrm{Cu}$ ions and possible mechanisms of action. Environmental pollution, 169, 81-89.

Brar, S. K., Verma, M., Tyagi, R. D., \& Surampalli, R. Y. (2010). Engineered nanoparticles in wastewater and wastewater sludge-Evidence and impacts. Waste Management, 30(3), 504520.

Bratby, J. (2006). Coagulation and flocculation in water and wastewater treatment (2 ed.). London: IWA.

Cao, H., \& Liu, X. (2010). Silver nanoparticles-modified films versus biomedical device-associated infections. Wiley Interdisciplinary Reviews: Nanomedicine and Nanobiotechnology, 2(6), 670-684.

CCME, C. C. (2010). A Review of the Current Canadian Legislative Framework for Wastewater Biosolids. PN 1446, ISBN 978-1-896997-95-7 PDF.

CCME, C. C. (2012). Guidance document for the beneficial use of municipal biosolids, municipal sludge and treated septage. PN 1473, ISBN 978-1-896997-85-8 PDF.

Chappell, M. A., Miller, L. F., George, A. J., Pettway, B. A., Price, C. L., Porter, B. E., . . Steevens, J. A. (2011). Simultaneous dispersion-dissolution behavior of concentrated silver 
nanoparticle suspensions in the presence of model organic solutes. Chemosphere, 84(8), 1108-1116.

Chen, Y., Wang, D., Zhu, X., Zheng, X., \& Feng, L. (2012). Long-term effects of copper nanoparticles on wastewater biological nutrient removal and $\mathrm{N}_{2} \mathrm{O}$ generation in the activated sludge process. Environmental Science \& Technology, 46(22), 12452-12458.

Cheng, Y., Yin, L., Lin, S., Wiesner, M., Bernhardt, E., \& Liu, J. (2011). Toxicity reduction of polymer-stabilized silver nanoparticles by sunlight. The Journal of Physical Chemistry, 115(11), 4425-4432.

Choi, O., \& Hu, Z. (2008). Size dependent and reactive oxygen species related nanosilver toxicity to nitrifying bacteria. Environmental Science \& Technology, 42(12), 4583-4588.

Chowdhuri, A., Gupta, V., Sreenivas, K., Kumar, R., Mozumdar, S., \& Patanjali, P. K. (2004). Response speed of $\mathrm{SnO} 2$-based $\mathrm{H} 2 \mathrm{~S}$ gas sensors with $\mathrm{CuO}$ nanoparticles. Applied Physics Letters, 84(7), 1180-1182.

City of Ottawa, C. o. (2014). City of Ottawa annual report. Ottawa, Canada: Citty of Ottawa.

Colman, B. P., Arnaout, C. L., Anciaux, S., Gunsch, C. K., Hochella Jr, M. F., Kim, B., . . al., e. (2013). Low Concentrations of Silver Nanoparticles in Biosolids Cause Adverse Ecosystem Responses under Realistic Field Scenario. PLOS One, 8(2), e57189.

Conway, J. R., Adeleye, A. S., Gardea-Torresdey, J., \& Keller, A. A. (2015). Aggregation, dissolution, and transformation of copper nanoparticles in natural waters. Environmental Science \& Technology, 49(5), 2749-2756.

Cunningham, S., Brennan-Fournet, M. E., Deirdre, L., Byrnes, L., \& Joshi, L. (2013). Effect of nanoparticle stabilization and physicochemical properties on exposure outcome: acute toxicity of silver nanoparticle preparations in zebrafish (Danio rerio). Environmental Science \& Technology, 47(8), 3883-3892.

Dentel, S. (2001). conditioning. In L. Spinosa, \& P. A. Vesilind (Eds.), Sludge into biosolids: processing, disposal, utilization (pp. 278-313). London: IWA.

Dhas, N. A., Raj, C. P., \& Gedanken, A. (1998). Synthesis, characterization, and properties of metallic copper nanoparticles. Chemistry of Materials, 10(5), 1446-1452. 
Dinesh, R., Anandaraj, M., Srinivasan, V., \& Hamza, S. (2012). Engineered nanoparticles in the soil and their potential implications to microbial activity. Geoderma, 173, 19-27.

Edwards, J. H., Thurlow, D. L., \& Ruf, M. E. (1992). Tillage and crop rotation effects on fertility status of a Hapludult soil. Soil Science Society of America Journal, 56(5), 1577-1582.

El Badawy, A. M., Silva, R. G., Morris, B., Scheckel, K. G., Suidan, M. T., \& Tolaymat, T. M. (2010). Surface charge-dependent toxicity of silver nanoparticles. Environmental Science \& Technology, 45(1), 283-287.

El-Nour, K. M., Eftaiha, A., Al-Warthan, A., \& Ammar, R. A. (2010). Synthesis and applications of silver nanoparticles. Arabian Journal of Chemistry, 3(3), 135-140.

EPA, U. E. (2018). Standards for the use or disposal of sewage sludge. Washington, DC, US: EPA40 CFR 503.

EPA, U. S. (2009). Targeted National Sewage Sludge Survey Sampling and Analysis Technical Report. Washington, DC: US Environmental Protection Agency Office of Water.

Fabrega, J., Renshaw, J. C., \& Lead, J. R. (2009). Interactions of silver nanoparticles with Pseudomonas putida biofilms. Environmental Science \& Technology, 43(23), 9004-9009.

Feng, Q. L., Wu, J., Chen, G., Cui, F., Kim, T., \& Kim, J. (2000). A mechanistic study of the antibacterial effect of silver ions on Escherichia coli and Staphylococcus aureus. Journal of Biomedical Materials Research, 52(4), 662-668.

Fierer, N., Jackson, J. A., Vilgalys, R., \& Jackson, R. B. (2005). Assessment of soil microbial community structure by use of taxon-specific quantitative PCR assays. Appl. Environ. Microbiol., 71(7), 4117-4120.

Gabbay, J., Borkow, G., Mishal, J., Magen, E., Zatcoff, R., \& Shemer-Avni, Y. (2006). Copper oxide impregnated textiles with potent biocidal activities. Journal of Industrial Textiles, $35(4), 323-335$.

Ganesh, R., Smeraldi, J., Hosseini, T., Khatib, L., Olson, B. H., \& Rosso, D. (2010). Evaluation of nanocopper removal and toxicity in municipal wastewaters. Environmental Science \& Technology, 44(20), 7808-7813. 
Geranio, L., Manfred, H., \& Bernd, N. (2009). The behavior of silver nanotextiles during washing. Environmental Science \& Technology, 43(21), 8113-8118.

Górska, E., Tudek, B., \& Russel, S. (2000). Degradation of cellulose by nitrogen-fixing strain of Bacillus polymyxa. Acta Microbiologica Polonica, 50(2), 129-137.

Gottschalk, F., Tobias, S., Roland W., S., \& Bernd, N. (2009). Modeled environmental concentrations of engineered nanomaterials ( $\mathrm{TiO} 2, \mathrm{ZnO}, \mathrm{Ag}, \mathrm{CNT}$, fullerenes) for different regions. Environmental Science \& Technology, 43(24), 9216-9222.

Hotze, E. M., Bottero, J.-Y., \& Wiesner, M. R. (2010). Theoretical framework for nanoparticle reactivity as a function of aggregation state. Langmuir , 26(13), 11170-11175.

Hu, W., Culloty, S., Darmody, G., Lynch, S., Davenport, J., Ramirez-Garcia, S., . . Sheehan, D. (2014). Toxicity of copper oxide nanoparticles in the blue mussel, Mytilus edulis: a redox proteomic investigation. Chemosphere, 108, 289-299.

Hugenholtz, P., Goebel, B. M., \& Pace, N. R. (1998). Impact of culture-independent studies on the emerging phylogenetic view of bacterial diversity. Journal of Bacteriology, 180(18), 47654774.

Impellitteri, C. A., Harmon, S., Silva, R. G., Miller, B. W., Scheckel, K. G., Luxton, T. P., . . . Panguluri, S. (2013). Transformation of silver nanoparticles in fresh, aged, and incinerated biosolids. Water Research, 47(12), 3878-3886.

Jang, S.-W., Oh, M.-S., Yang, S. I., \& Cho, E.-M. (2016). Gene expression profiles of human neuroblastoma cells exposed to $\mathrm{CuO}$ nanoparticles and $\mathrm{Cu}$ ions. BioChip Journal, 10(2), 140-149.

Janssen, P. H. (2006). Identifying the dominant soil bacterial taxa in libraries of $16 \mathrm{~S}$ rRNA and 16S rRNA genes. Applied and Environmental Microbiology, 72(3), 1719-1728.

Jo, H. J., Choi, J. W., Lee, S. H., \& Hong, S. W. (2012). Acute toxicity of Ag and CuO nanoparticle suspensions against Daphnia magna: the importance of their dissolved fraction varying with preparation methods. Journal of Hazardous Materials , 227, 301-308.

Johnston, H. J., Hutchison, G., Christensen, F. M., Peters, S., Hankin, S., \& Stone, V. (2010). A review of the in vivo and in vitro toxicity of silver and gold particulates: particle attributes 
and biological mechanisms responsible for the observed toxicity. Critical Reviews in Toxicology , 40(4), 328-346.

Jomova, K., \& Valko, M. (2011). Advances in metal-induced oxidative stress and human disease. Toxicology, 283(2-3), 65-87.

Juling, S., Niedzwiecka, A., Böhmert, L., Lichtenstein, D., Selve, S., Braeuning, A., . . Lampen, A. (2017). Protein corona analysis of silver nanoparticles links to their cellular effects. Journal of Proteome Research, 16(11), 4020-4034.

Kaegi, R., Sinnet, B., Zuleeg, S., Hagendorfer, H., Mueller, E., Vonbank, R., . . Burkhardt, M. (2010). Release of silver nanoparticles from outdoor facades. Environmental Pollution, 158(9), 2900-2905.

Kaegi, R., Voegelin, A., Ort, C., Sinnet, B., Thalmann, B., Krismer, J., . . Mueller, E. (2013). Fate and transformation of silver nanoparticles in urban wastewater systems. Water Research, 47(12), 3866-3877.

Kaegi, R., Voegelin, A., Sinnet, B., Zuleeg, S., Hagendorfer, H., Burkhardt, M., \& Siegrist, H. (2011). Behavior of metallic silver nanoparticles in a pilot wastewater treatment plant. Environmental Science \& Technology, 45(9), 3902-3908.

Kawata, K., Osawa, M., \& Okabe, S. (2009). In vitro toxicity of silver nanoparticles at noncytotoxic doses to HepG2 human hepatoma cells. Environmental Science \& Technology, 43(15), 6046-6051.

Keller, A. A., McFerran., S., Lazareva, A., \& Suh, S. (2013). Global life cycle releases of engineered nanomaterials. Journal of Nanoparticle Research, 15(6), 1692.

Khan, S. S., Mukherjee, A., \& Chandrasekaran, N. (2011). Impact of exopolysaccharides on the stability of silver nanoparticles in water. Water Research, 45(16), 5184-5190.

Kim, B., Murayama, M., Colman, B. P., \& Hochella, M. F. (2012). Characterization and environmental implications of nano-and larger TiO 2 particles in sewage sludge, and soils amended with sewage sludge. Journal of Environmental Monitoring, 14(4), 1128-1136. 
Kim, B., Park, C.-S., Murayama, M., \& Hochella Jr., M. F. (2010). Discovery and characterization of silver sulfide nanoparticles in final sewage sludge products. Environmental Science \& Technology , 44(19), 7509-7514.

Kittler, S., Greulich, C., Diendorf, J., Koller, M., \& Epple, M. (2010). Toxicity of silver nanoparticles increases during storage because of slow dissolution under release of silver ions. Chemistry of Materials, 22(16), 4548-4554.

Klasen, H. J. (2000). A historical review of the use of silver in the treatment of burns. Burns, 26(2), $131-138$

Kumaran, R. S., Choi, Y.-K., Kim, H. J., \& Kim, K. J. (2014). Quantitation of oxidative stress gene expression in MCF-7 human cell lines treated with water-dispersible $\mathrm{CuO}$ nanoparticles. Applied Biochemistry and Biotechnology, 173(3), 731-740.

Kurtböke, D. I. (2012). Biodiscovery from rare actinomycetes: an eco-taxonomical perspective. Applied Microbiology and Biotechnology, 93(5), 1843-1852.

Kvitek, L., Panáček, A., Soukupova, J., Kolář, M., Večeřová, R., Prucek, R., . . Zbořil, R. (2008). Effect of surfactants and polymers on stability and antibacterial activity of silver nanoparticles (NPs). The Journal of Physical Chemistry C, 112(15), 5825-5834.

Lankveld, D. P., Oomen, A. G., Krystek, P., Neigh, A. T.-d., Noorlander, C., Van Eijkeren, J., .. . De Jong, W. (2010). The kinetics of the tissue distribution of silver nanoparticles of different sizes. Biomaterials, 31(32), 8350-8361.

Lansdown, A. B. (2004). A review of the use of silver in wound care: facts and fallacies. British Journal of Nursing, 13(1), S6-S19.

Larguinho, M., \& Baptista, P. V. (2012). Gold and silver nanoparticles for clinical diagnosticsfrom genomics to proteomics. Journal of Proteomics, 75(10), 2811-2823.

Lee, Y.-J., Kim, J., Oh, J., Bae, S., Lee, S., Hong, I. S., \& Kim, S.-H. (2012). Ion-release kinetics and ecotoxicity effects of silver nanoparticles. Environmental Toxicology and Chemistry, $31(1), 155-159$. 
Levard, C., Reinsch, B. C., Michel, F. M., Oumahi, C., Lowry, G. V., \& Brown Jr., G. E. (2011). Sulfidation processes of PVP-coated silver nanoparticles in aqueous solution: impact on dissolution rate. Environmental Science \& Technology, 45(12), 5260-5266.

Li, F., Zhou, X., Zhu, J., Ma, J., Huang, X., \& Wong, S. T. (2007). High content image analysis for human $\mathrm{H} 4$ neuroglioma cells exposed to $\mathrm{CuO}$ nanoparticles. BMC Biotechnology, 7(1), 66.

Li, L., Hartmann, G., Döblinger, M., \& Schuster, M. (2013). uantification of nanoscale silver particles removal and release from municipal wastewater treatment plants in Germany. Environmental Science \& Technology, 47(13), 7317-7323.

Li, L., Stoiber, M., Wimmer, A., Xu, Z., Lindenblatt, C., Helmreich, B., \& Schuster, M. (2016). To what extent can full-scale wastewater treatment plant effluent influence the occurrence of silver based nanoparticles in surface waters? Environmental Science \& Technology.

Li, X., Lenhart, J. J., \& Walker, H. W. (2010). Dissolution-accompanied aggregation kinetics of silver nanoparticles. Langmuir, 26(22), 16690-16698.

Liu, J., \& Hurt, R. H. (2010). Ion release kinetics and particle persistence in aqueous nano-silver colloids. Environmental Science \& Technology, 44(6), 2169-2175.

Liu, J., Sonshine, D. A., Shervani, S., \& Hurt, R. H. (2010). Controlled release of biologically active silver from nanosilver surfaces. ACS Nano , 4(11), 6903-6913.

Liu, P., Guan, R., Ye, X., Jiang, J., Liu, M., Huang, G., \& Chen, X. (2011). Toxicity of nano-and micro-sized silver particles in human hepatocyte cell line L02. Journal of Physics: Conference Series, 304(1), 012036.

Lorenz, C., Hagendorfer, H., Goetz, N. v., Kaegi, R., Gehrig, R., Ulrich, A., . . Hungerbühler, K. (2011). Nanosized aerosols from consumer sprays: experimental analysis and exposure modeling for four commercial products. Journal of Nanoparticle Research, 8(13), 33773391.

Lowry, G. V., Gregory, K. B., Apte, S. C., \& Lead, J. R. (2012). Transformations of nanomaterials in the environment. Environmental Science and Technology, 46, 6893-6899. 
Ma, R., Levard, C., Judy, J. D., Unrine, J. M., Durenkamp, M., Martin, B., . . Lowry, G. V. (2013). Fate of zinc oxide and silver nanoparticles in a pilot wastewater treatment plant and in processed biosolids. Environmental Science \& Technology, 48(1), 104-112.

Madigan, M., \& Martinko, J. (2005). Brock Biology of Microorganisms (11 ed.). NJ: Prentice hall: Upper Saddle River.

Masrahi, A., VandeVoort, A. R., \& Arai, Y. (2014). Effects of silver nanoparticle on soilnitrification processes. Archives of Environmental Contamination and Toxicology, 66(4), 504-513.

Metcalf, L., \& Eddy, H. P. (2003). Waste Water Engineering: Treatment and Reuse (4 ed.). New York: McGraw-Hill.

Miao, L., Wang, C., Hou, J., Wang, P., Ao, Y., Li, Y., . . Xu, Y. (2015). Enhanced stability and dissolution of $\mathrm{CuO}$ nanoparticles by extracellular polymeric substances in aqueous environment. Journal of Nanoparticle Research, 17(10), 1-12.

Mihranyan, A., Ferraz, N., \& Strømme, M. (2012). Current status and future prospects of nanotechnology in cosmetics. Progress in Materials Science, 57(5), 875-910.

Morones, J. R., Elechiguerra, J. L., Holt, A. H., Holt, K., Kouri, J. B., Ramírez, J. T., \& Yacaman, M. J. (2005). The bactericidal effect of silver nanoparticles. Nanotechnology , 16(10), 2346.

Morris, J., \& Willis, J. (2007). US Environmental Protection Agency nanotechnology white paper. Washington, DC: US Environmental Protection Agency.

Mueller, N. C., \& Nowack, B. (2008). Exposure modeling of engineered nanoparticles in the environment. Environmental Science and Technology, 42(12), 4447-4453.

Nielsen, A. H., Vollertsen, J., Jensen, H. S., Madsen, H. I., \& Hvitved-Jacobsen, T. (2006). Aerobic and anaerobic transformations of sulfide in a sewer system-field study and model simulations. Proceedings of the Water Environment Federation, 9, 3654-3670.

Nowack, B., \& Bucheli, a. T. (2007). Occurrence, behavior and effects of nanoparticles in the environment. Environmental Pollution, 150(1), 5-22. 
OMAFRA, O. M. (2019, May 14). Information on Analysis Requirements for Non-Agricultural Source Materials. Retrieved July 19, 2019, from http://www.omafra.gov.on.ca/english/nm/nasm/labs.htm

Pal, S., Tak, Y. K., \& Song, J. M. (2007). Does the antibacterial activity of silver nanoparticles depend on the shape of the nanoparticle? A study of the gram-negative bacterium Escherichia coli. Applied and Environmental Microbiology, 73(6), 1712-1720.

Panacek, A., Kolar, M., Vecerova, R., Prucek, R., Soukupova, J., Krystof, V., . . Kvitek, L. (2009). Antifungal activity of silver nanoparticles against Candida spp. Biomaterials, 30(31), 6333-6340.

Philip, H., Christian, P., Karen L., H., \& Norman R., P. (1998). Novel division level bacterial diversity in a Yellowstone hot spring. Bacteriol Journal, 180(2), 366-376.

Powers, C. M., Badireddy, A. R., Ryde, I. T., Seidler, F. J., \& Slotkin, T. A. (2011). Silver nanoparticles compromise neurodevelopment in PC12 cells: critical contributions of silver ion, particle size, coating, and composition. Environmental Health Perspectives, 119(1), 37.

Qu, F., Xu, H., Wei, H., La, W., Xiong, Y., Xu, F., . . Wang, Y. A. (2010). Effects of pH and temperature on antibacterial activity of silver nanoparticles. Biomedical Engineering and Informatics (pp. 2033-2037). IEEE.

Rai, M., Yadav, A., \& Gade, A. (2009). Silver nanoparticles as a new generation of antimicrobials. Biotechnology Advances, 27(1), 76-83.

Ramyadevi, J., Jeyasubramanian, K., Marikani, A., Rajakumar, G., \& Rahuman, A. A. (2012). Synthesis and antimicrobial activity of copper nanoparticles. Materials Letters, 71, 114116.

Reidy, B., Haase, A., Luch, A., Dawson, K., \& Lynch, I. (2013). Mechanisms of silver nanoparticle release, transformation and toxicity: a critical review of current knowledge and recommendations for future studies and applications. Materials, 6(6), 2295-2350. 
Rotoli, B. M., Bussolat, O., Costa, A. L., Blosi, M., Cristo, L. D., Zanello, P. P., . . Bergamaschi, E. (2012). Comparative effects of metal oxide nanoparticles on human airway epithelial cells and macrophages. Journal of Nanoparticle Research, 14(9), 1069.

Samberg, M. E., Orndorff, P. E., \& Monteiro-Riviere, N. A. (2011). Antibacterial efficacy of silver nanoparticles of different sizes, surface conditions and synthesis methods. Nanotoxicology, $5(2), 244-253$.

Shafer, M. M., Joel T., O., \& David E., A. (1998). Removal, partitioning, and fate of silver and other metals in wastewater treatment plants and effluent-receiving streams. Environmental Toxicology and Chemistry, 17(4), 630-641.

Shoults-Wilson, W. A., Reinsch, B. C., Tsyusko, O. V., Bertsch, P. M., Lowry, G. V., \& Unrine, J. M. (2011). Role of particle size and soil type in toxicity of silver nanoparticles to earthworms. Soil Science Society of America Journal, 75(2), 365-377.

Siddiqui, M. A., Alhadlaq, H. A., Ahmad, J., Al-Khedhairy, A. A., Musarrat, J., \& Ahamed, M. (2013). Copper oxide nanoparticles induced mitochondria mediated apoptosis in human hepatocarcinoma cells. PloS one 8, no. 8 (2013): e69534, 8(8), e69534.

Song, L., Vijver, M. G., Peijnenburg, W. J., Galloway, T. S., \& Tyler, C. R. (2015). A comparative analysis on the in vivo toxicity of copper nanoparticles in three species of freshwater fish. Chemosphere, 139, 181-189.

Suresh, A. K., Pelletier, D. A., Wang, W., Morrell-Falvey, J. L., Gu, B., \& Doktycz, M. J. (2012). Cytotoxicity induced by engineered silver nanocrystallites is dependent on surface coatings and cell types. Langmuir, 28(5), 2727-2735.

Thomas, F., Hehemann, J., Rebuffet, E., Czjzek, M., \& Michel, G. (2011). Environmental and gut bacteroidetes: the food connection. In Human health and disease in a microbial world (p. 96).

Tong, Z., Bischoff, M., Nies, L., Applegate, B., \& Turco, R. F. (2007). Impact of fullerene (C60) on a soil microbial community. Environmental Science \& Technology , 41(8), 2985-2991.

Turovskiy, I. S., \& Mathai, P. K. (2006). Wastewater Sludge Processing . New Jersey: John Wiley and Sons. 
US Research Nanomaterials, I. (2017). US Research Nanomaterials Inc. Retrieved December 5, 2017, from https://www.us-nano.com/inc/sdetail/602

Vesilind, P. A., Hartman, G. C., \& Skene, E. T. (1986). Sludge management and disposal for the practicing engineer (1st ed.). Chelsea, Michigan: Lewis Publishers, Inc.

Wang, D., \& Chen, Y. (2016). Critical review of the influences of nanoparticles on biological wastewater treatment and sludge digestion. Critical Reviews in Biotechnology, 35(5), 816828.

Wang, Z., Bussche, A. V., Kabadi, P. K., Kane, A. B., \& Hurt, R. H. (2013). Biological and environmental transformations of copper-based nanomaterials. ACS nano, 7(10), 87158727.

Wei, Y., Chen, S., Kowalczyk, B., Huda, S., Gray, T. P., \& Grzybowski, B. A. (2010). Synthesis of stable, low-dispersity copper nanoparticles and nanorods and their antifungal and catalytic properties. The Journal of Physical Chemistry, 114(37), 15612-15616.

Wiesner, M. R., \& Plata, L. D. (2012). Environmental, health and safety issues: Incinerator filters nanoparticles. Nature Nanotechnology, 7(8), 487.

Wiesner, M. R., Lowry, G. V., Jones, K. L., Hochella, M. F., Giulio, R. T., Casman, E., \& Bernhardt, E. S. (2009). Decreasing uncertainties in assessing environmental exposure, risk, and ecological implications of nanomaterials. Environmental Science \& Technology, 43, 6458-6462.

Xiu, Z.-m., Zhang, Q.-b., Puppala, H. L., Colvin, V. L., \& Alvarez, P. J. (2012). Negligible particlespecific antibacterial activity of silver nanoparticles. Nano Letters, 8(12), 4271-4275.

Xu, L., Li, X., Takemura, T., Hanagata, N., Wu, G., \& Chou, L. L. (2012). Genotoxicity and molecular response of silver nanoparticle (NP)-based hydrogel. Journal of Nanobiotechnology, 10(1), 16.

Yang, X., Gondikas, A. P., Marinakos, S. M., Auffan, M., Liu, J., Hsu-Kim, H., \& Meyer, J. N. (2011). Mechanism of silver nanoparticle toxicity is dependent on dissolved silver and surface coating in Caenorhabditis elegans. Environmental Science \& Technology , 46(2), $1119-1127$. 
Yang, Z., Liu, Z., Allaker, R., Reip, P., Oxford, J., Ahmad, Z., \& Reng, G. (2010). A review of nanoparticle functionality and toxicity on the central nervous system. Interface Focus, 7 , S411-S422. 


\section{Chapter 3: Materials and Methods}

\subsection{Silver and copper oxide nanoparticles}

Two different nanoparticles were used in this research: silver nanoparticles (AgNPs) and copper oxide nanoparticles (CuONPs). A stock suspension of pristine AgNPs in water with a purity of $99.9 \%$ was purchased from nanoComposix Inc. (San Diego, CA, USA). The particle size distribution of AgNPs was examined and confirmed by transmission electron microscopy (TEM) analysis. AgNPs suspension stock solution was maintained at $4^{\circ} \mathrm{C}$ until used. A stock solution of CuONPs with $99.95 \%$ purity was purchased from the US Research Nanomaterials Inc (Houston, USA) and stored at $4^{\circ} \mathrm{C}$ until use. Both AgNPs and CuONPs are insoluble in water. Table 3-1 shows the characteristics of AgNPs and CuONPs.

Table 3-1 AgNPs and CuONPs characteristics.

\begin{tabular}{|l|l|l|}
\hline Parameter & AgNPs & CuONPs \\
\hline Diameter (TEM) & $23.1 \pm 6.9 \mathrm{~nm}$ & $25-55 \mathrm{~nm}$ \\
\hline Morphology & Nearly spherical & Nearly spherical \\
\hline pH of the solution & 6.3 & $7-9$ \\
\hline Zeta potential & $-30.6 \mathrm{mV}$ & - \\
\hline Particle surface coating & polyvinylpyrrolidone (PVP) & None \\
\hline Solvent & Milli-Q water & Milli-Q water \\
\hline
\end{tabular}

\subsection{Sludge samples}

Three different types of wastewater sludges were used in this research; anaerobically digested sludge, primary sludge and thickened waste activated sludge. Sludge samples were collected from the Robert O. Pickard Environmental Center (ROPEC), a wastewater treatment plant located in Ottawa, Canada. It receives approximately $390 \mathrm{ML} / \mathrm{d}$ of 
wastewater (City of Ottawa, 2014) from residential and commercial sources in the Ottawa region. Sludge samples were collected and used on the day of each experiment or stored at $4 \pm 0.2^{\circ} \mathrm{C}$ until use for up to four days and they were used directly without filtration. Anaerobically digested sludge, primary sludge and thickened waste activated sludge (tWAS) were collected from the mesophilic anaerobic digesters, the primary clarifier and the thickener of the waste activated sludge, respectively. An example of the sludge properties used in this study is provided in Table 3-2.

Table 3-2 Characteristics of raw sludge samples.

\begin{tabular}{|l|l|l|l|}
\hline Properties & $\begin{array}{l}\text { Anaerobically } \\
\text { digested sludge }\end{array}$ & Primary sludge & tWAS sludge \\
\hline Total solids (TS) $(\mathrm{g} / \mathrm{kg})$ & $19.2 \pm 1.3$ & $42.7 \pm 7.2$ & $52.7 \pm 1.6$ \\
\hline Volatile solids (VS) $(\mathrm{g} / \mathrm{kg})$ & $14.9 \pm 2.1$ & $28.7 \pm 3.5$ & $38.6 \pm 1.5$ \\
\hline $\begin{array}{l}\text { Total chemical oxygen demand } \\
\text { (tCOD) }(\mathrm{g} / \mathrm{kg})\end{array}$ & $19.8 \pm 1.1$ & $52.0 \pm 8.3$ & $73.9 \pm 2.1$ \\
\hline $\begin{array}{l}\text { Soluble oxygen demand (sCOD) } \\
\text { (g/kg) }\end{array}$ & $8.4 \pm 0.2$ & $2.0 \pm 1.1$ & $8.2 \pm 0.1$ \\
\hline $\mathrm{pH}$ & $7.4 \pm 0.1$ & $7.6 \pm 0.1$ & $7.0 \pm 0.1$ \\
\hline
\end{tabular}

Values are the mean of three replicate measurements \pm standard deviation.

\subsection{Soil samples}

For this research, loamy topsoil without additives such as compost or chemical fertilizer was obtained from Artistic Landscape Designs Ltd. Soil samples were sieved through a 2 mm sieve, air dried, and stored at $4^{\circ} \mathrm{C}$ until use. The soil was analyzed for total organic carbon (TOC), total nitrogen (TN) and total phosphorus (TP) using $0.01 \mathrm{M}$ calcium chloride as an extraction reagent (Houba et al., 2000). The method involved mixing the soil sample into a volume of extraction reagent at a 1:10 (W: V) (soil: extraction reagent ratio), shaking the mixture for two hours at $20{ }^{\circ} \mathrm{C}$ then centrifuging for $12 \mathrm{~min}$ at $1800 \times \mathrm{g}$. The 
analyses were done when required according to methods described in Section 3.8. The reported soil $\mathrm{pH}$ was measured by suspending equal amounts of water and soil at a 1:1 ratio (1 g soil: $1 \mathrm{~mL}$ water) (Gregorich \& Carter, 2007), and the cation exchange capacity (CEC) of the soil was measured according to the method reported by Rebecca (2004). Table 3-3 shows the soil characteristics.

Table 3-3 Soil sample characteristics.

\begin{tabular}{|l|l|}
\hline TOC & $4248 \pm 12 \mathrm{mg} / \mathrm{kg}$ \\
\hline TN & $184 \pm 7 \mathrm{mg} / \mathrm{kg}$ \\
\hline TP & $13 \pm 2 \mathrm{mg} / \mathrm{kg}$ \\
\hline pH & $6.6 \pm 0.7$ \\
\hline CEC & $30.8 \pm 2 . \mathrm{meq} / 100 \mathrm{~g}$ \\
\hline
\end{tabular}

Values are the mean of three replicate measurements \pm standard deviation.

\subsection{Conditioning and treatment chemicals}

Ferric chloride $\left(\mathrm{FeCl}_{3}\right)$ and aluminum sulfate $\left(\mathrm{Al}_{2}\left(\mathrm{SO}_{4}\right)_{3} \bullet(14-18) \mathrm{H}_{2} \mathrm{O}\right)$ were used as inorganic conditioning agents. Ferric chloride was purchased from Fisher Scientific as a powder, and a stock solution was prepared at a concentration of $40 \mathrm{mg} / \mathrm{mL}(4 \%)$. Aluminum sulfate was purchased from Anachemia Canada Inc. in granular form, and a stock solution was prepared at a concentration of $170 \mathrm{mg} / \mathrm{mL}$ (17\%). FloPolymer CA 4600 (SNF Canada) was used in this study as the organic coagulant to condition the anaerobically digested sludge. CA 4600 is a dry cationic polyacrylamide polymer that has high charge density and high molecular weight. A stock solution of the polymer was prepared at a concentration of $2 \mathrm{mg} / \mathrm{mL}(0.2 \%)$ by fast mixing for $1 \mathrm{~h}$ with a magnetic stirrer followed by slow mixing for $1 \mathrm{~h}$ and then resting for $1 \mathrm{~h}$ before use. Quicklime $(\mathrm{CaO})$, with a molecular weight of $56.1 \mathrm{~g} / \mathrm{mol}$, was purchased from Sigma-Aldrich for the study. Lime 
slurry was prepared by mixing powdered lime with water at a concentration of $150 \mathrm{mg} / \mathrm{mL}$ $(15 \%)$. The slurry was then mixed with anaerobically digested sludge at a concentration that maintained $\mathrm{pH}$ above 12 for $2 \mathrm{~h}$.

\subsection{Phosphate buffered saline}

Phosphate buffered saline (PBS) was used to wash the soil and sludge samples before DNA extraction. The PBS was prepared by adding $1.25 \mathrm{~mL}$ phosphate buffer stock solution and $5 \mathrm{~mL}$ magnesium chloride solution to $1 \mathrm{~L}$ deionized water. Phosphate buffer stock solution was prepared by dissolving $34.0 \mathrm{~g}$ potassium dihydrogen phosphate $\left(\mathrm{KH}_{2} \mathrm{PO}_{4}\right)$ in $500 \mathrm{~mL}$ deionized water, adjusting the $\mathrm{pH}$ of the solution to $7.2 \pm 0.5$ with $1 \mathrm{~N}$ sodium hydroxide $(\mathrm{NaOH})$ and completing the volume of the solution to $1 \mathrm{~L}$. Magnesium chloride solution was prepared by dissolving $81.1 \mathrm{~g} \mathrm{MgCl}_{2} \cdot 6 \mathrm{H}_{2} \mathrm{O}$ in $1 \mathrm{~L}$ deionized water.

\subsection{Quantification and characterization of nanoparticles}

Size and shape distribution of AgNPs were examined using two main visualization methods: transmission electron microscopy (TEM) and scanning electron microscopy (SEM). These are powerful techniques that can be used to study the aggregation, size, structure and shape of the nanoparticles (Mavrocordatos et al., 2004). In addition, these methods were accompanied by energy-dispersive x-ray spectroscopy (EDS) that provides chemical information such as the elemental composition of a specific part from a sample (Baalousha et al., 2011). Concentrations of the nanoparticles were assessed using a single particle inductively coupled plasma mass spectrometry (SP-ICP-MS) analytical tool. ICPMS provides the elemental composition and concentration of colloidal solutions (Beermann et al., 2007), and, if it is combined with more advanced analytical procedures 
such as operating in a single-particle counting mode (SP-ICP-MS), it can distinguish between the dissolved and particulate portions of an element (Pace et al., 2011). Thus, it can measure both the concentration of the nanoparticles and the total concentration of the element and has the ability to differentiate the solid nanoparticles from those that are dissolved.

\subsubsection{Transmission electron microscopy}

Particle size, shape and morphology were examined using TEM. The samples were prepared by placing approximately $5 \mu \mathrm{L}$ of the conditioned sludge onto a $3.05 \mu \mathrm{m}$ support copper grid film (copper grid film is coated with formvar/carbon, with a 200 mesh, and hole size of approximately $97 \mathrm{~nm}$ from Ted Pella Inc.). After preparation, the samples were taken for analysis to the nano imaging facility (NIF) at Carleton University. TEM is an analytical tool that provides visual characteristics of nanoparticles below the nm range. TEM also provides information on the aggregation, size, structure, shape, and dispersion of nanoparticles (Mavrocordatos et al., 2004). TEM operates by sending an electron beam from a source that enters the very thin sample and interacts with the sample through scattering as it passes through the sample. The electrons that are not absorbed are then focused by an objective lens and amplified by a magnifying lens to produce an image.

\subsubsection{Energy-dispersive $x$-ray spectroscopy}

In addition to morphological analyses, the elemental composition of the samples was analyzed twice using EDS along with TEM and SEM. When combined with these imaging tools, EDS can provide the elemental composition of an area as small as a few nanometers in diameter. EDS makes use of the x-ray produced from a sample subjected to an electron 
beam to characterize the elemental composition of the sample through its energy spectrum display. The results are shown as a plot of the emitted x-rays versus their energy in $\mathrm{keV}$. The generated plot has many peaks, and each peak corresponds to a specific element.

\subsubsection{Single particle inductively coupled plasma mass spectrometry}

The concentration of total and dissolved silver was measured using SP-ICP-MS. Samples were prepared by filtering (volume of sample) through $0.45 \mu \mathrm{m}$ nylon filter before injection into the SP-ICP-MS instrument. ICP-MS is a powerful tool that can determine the metal and non-metal elemental composition of a liquid sample at very low concentration; as low as parts per trillion if non-interfered low background isotopes are used (Thomas, 2013). Unlike ICP-MS, SP-ICP-MS can distinguish between the particulate and dissolved portions of a metal (Pace et al., 2011; Laborda et al., 2014). SP-ICP-MS works through the injection of a liquid sample to the neutralizer that converts the sample into an aerosol with argon, and the aerosol is then introduced into the plasma where ions are created (EspinosaOrtiz \& Vaca-Mier, 2013).

\subsection{Microbiological analyses}

This research investigated the bacterial population and diversity change in sludge and soil after exposure to AgNPs or CuONPs. This was accomplished using two molecular biology techniques, quantitative polymerase chain reaction (qPCR) and next-generation sequencing. In addition, Live/Dead staining and heterotrophic bacteria counts were investigated as part of the analyses. 


\subsubsection{DNA extraction and quantification}

Total genomic DNA was extracted using the DNeasy PowerSoil Pro DNA Isolation Kit from MO BIO Laboratories Inc. (QIAGEN Online Shop). In most cases, the samples were prewashed before the exaction according to a method described by Kibbee \& Ormeci (2017). The DNA extraction was done according to the instruction manual of the isolation kit, except that all incubation periods were doubled from five to ten minutes. The concentration of extracted DNA was measured using Qubit ${ }^{\circledR}$ dsDNA HS Assay Kits with the Qubit 3.0 Fluorometer from Molecular Probes, Life Technology, Eugene, OR. The DNA samples were stored at $-20^{\circ} \mathrm{C}$ until they were used for bacterial population and diversity analysis.

\subsubsection{Bacterial population analysis}

Bacterial population and diversity were investigated by DNA analysis using either qPCR or next-generation sequences. The bacterial population and diversity methods are explained in the materials and methods section of each chapter.

\subsubsection{Studied phyla in soil}

In Chapter 4 and 5, overall phyla presented and detected were analyzed. In Chapters 6 and 7, the five most prevalent bacterial phyla found in soil and sludge were selected for investigation: Acidobacteria, Actinobacteria, Bacteroidetes, Firmicutes and Proteobacteria. These phyla were chosen because they are the most relevant bacteria in soil environments pertaining to plant health and overall soil health. Individual qPCR protocols were developed using primers which were selected based on the latest published studies to 
target the major phylogenetic diversity for each selected phylum. The primers were purchased from Life Technologies (Burlington, ON). Primers references and properties are shown in Table 3-4. DNA was extracted from each soil reactor in duplicate and used as template DNA in each phylum specific qPCR.

\subsubsection{2 qPCR protocols}

qPCR was performed to assess the relative abundance of the five studied phyla in the soil samples. The qPCR runs were conducted in 96-well plates using the CFX96 Real-Time PCR Detection System from BioRad. The qPCR reaction volume of $25 \mu \mathrm{L}$ consisted of 12.5 $\mu \mathrm{L}$ qPCR buffer, $0.5 \mu \mathrm{L}(200 \mathrm{nM})$ forward primer, $0.5 \mu \mathrm{L}(200 \mathrm{nM})$ reverse primer, a volume of DNA template equilibrated to $30 \mathrm{ng}$ concentration, and the required water to attain $25 \mu \mathrm{L}$, final reaction volume. Each plate contained three replicate reactions for each DNA sample and three replicate reactions for appropriate standards. The qPCR protocol was the same for all the studied phyla except for the annealing temperature, which was different for each, as shown in Table 3-4. The qPCR protocol entailed an initial denaturation step at $95^{\circ} \mathrm{C}$ for $5 \mathrm{~min}, 40$ cycles at $95^{\circ} \mathrm{C}$ for $10 \mathrm{~s}$, annealing for $20 \mathrm{~s}$, an extension at $72^{\circ} \mathrm{C}$ for $15 \mathrm{~s}$, and a melt curve from 65 to $95^{\circ} \mathrm{C}$ with a $0.5^{\circ} \mathrm{C}$ increase every 5

s. The qPCR master mix was SsoFast EvaGreen Supermix from Bio-Rad Laboratories Ltd, Canada and it contained dNTPs, Sso7d fusion polymerase, $\mathrm{MgCl}_{2}$, EvaGreen dye, and stabilizers. Preliminary experiments were conducted to optimize the annealing temperature for each phylum protocol. The annealing temperature was determined using the extracted soil DNA as a template in a thermal gradient qPCR reaction, with different annealing temperature ranges for each phylum. Melt curve analysis of the PCR products was 
performed after each assay to confirm that the fluorescence signal originated from specific PCR products, rather than excess primer-dimers or non-specific amplification.

Table 3-4 Phyla primer pairs used in qPCR assay.

\begin{tabular}{|c|c|c|c|c|c|}
\hline Phylum & $\begin{array}{l}\text { Amplicon } \\
\text { size (bp) }\end{array}$ & $\begin{array}{l}\text { Primer } \\
\text { name }\end{array}$ & Primer sequence $\left(5^{\prime} \rightarrow 3^{\prime}\right)$ & $\begin{array}{l}\text { Annealing } \\
\text { temperature } \\
\left({ }^{\circ} \mathrm{C}\right)\end{array}$ & References \\
\hline \multirow{2}{*}{ Acidobacteria } & \multirow{2}{*}{500} & fAcid31 & GATCCTGGCTCAGAATC & \multirow{2}{*}{55.9} & \multirow{2}{*}{$\begin{array}{l}\text { (Fierer et al., } \\
2005 \text { ) }\end{array}$} \\
\hline & & rEub518 & ATTACCGCGGCTGCTGG & & \\
\hline \multirow{2}{*}{ Actinobacteria } & \multirow{2}{*}{166} & fActi & GRDACYGCCGGGGTYAACT & \multirow{2}{*}{57.2} & \multirow{2}{*}{$\begin{array}{l}\text { (Pfeiffer et al., } \\
\text { 2014) }\end{array}$} \\
\hline & & rActi & TCWGCGATTACTAGCGAC & & \\
\hline \multirow{2}{*}{ Bacteroidetes } & \multirow{2}{*}{181} & fBdet & GCACGGGTGMGTAACRCGTACCCT & \multirow{2}{*}{61} & \multirow{2}{*}{$\begin{array}{l}\text { (Pfeiffer et al., } \\
\text { 2014) }\end{array}$} \\
\hline & & rBdet & GTRTCTCAGTDCCARTGTGGG & & \\
\hline \multirow{2}{*}{ Firmicutes } & \multirow{2}{*}{156} & fFirm & CAGCAGTAGGGAATCTTC & \multirow{2}{*}{55.3} & \multirow{2}{*}{$\begin{array}{l}\text { (Pfeiffer et al., } \\
\text { 2014) }\end{array}$} \\
\hline & & rFirm & ACCTACGTATTACCGCGG & & \\
\hline \multirow{2}{*}{ Proteobacteria } & \multirow{2}{*}{140} & $767 \mathrm{fProt}$ & AAGCGTGGGGAGCAAACA & \multirow{2}{*}{54.8} & \multirow{2}{*}{$\begin{array}{l}\text { (Morales \& } \\
\text { Holben, 2009) }\end{array}$} \\
\hline & & 907rProt & CCGTCAATTCMTTTRAGTTT & & \\
\hline \multirow{2}{*}{$\begin{array}{l}16 \mathrm{~S} \\
\text { (any bacteria) }\end{array}$} & \multirow{2}{*}{180} & $338 \mathrm{~F}$ & ACT CCT ACG GGA GGC AGC AG & \multirow{2}{*}{61.9} & \multirow{2}{*}{$\begin{array}{l}\text { (Shakya et al., } \\
\text { 2013) }\end{array}$} \\
\hline & & $518 \mathrm{R}$ & ATT ACC GCG GCT GCT GG & & \\
\hline
\end{tabular}

\subsubsection{3 qPCR results analysis}

Universal 16S ribosomal RNA primers were used to amplify a $\sim 180$ bp sequence which was used to calculate the baseline $16 \mathrm{~S}$ gene copy number which was compared to each of the five phyla specific calculated gene copy number. The data generated relative population trends for each phylum over time. The data analysis was based on three main fundamental assumptions:

1. $\mathrm{Cq}$ is equivalent to $3.33 \log _{10}$ (target concentration).

Many studies have used a control curve to relate the number (concentration) of DNA in the qPCR product to the corresponding Cq. Svec et al. (2015) have mentioned that constructing a control curve with $\log _{10}$ of DNA number versus Cq (resulted from the qPCR run of serial concentrations of DNA) will be linear with a slope of 3.33 when the qPCR efficiency is $100 \%$ (Svec et al., 2015). 
2. Every bacterium has $1 \sim$ fg of DNA.

The concentration of DNA template that was used in the qPCR reaction is $30 \mathrm{ng}$. The qPCR analysis in this study relies on a relative estimation of the bacterial population. If it is assumed that every bacterium has $1 \sim \mathrm{fg}$ of DNA, then $30 \mathrm{ng}$ of DNA is equal to $3 \times 10^{7}$ bacteria. This is a relative estimation because the soil extracted DNA belongs to many other living things besides bacteria such as plants and eukaryotes.

3. Every phylum has a specific copy number of $16 \mathrm{~S}$ rRNA.

Based on the recent study by (Větrovský \& Baldrian, 2013), each phylum has a specific copy number of $16 \mathrm{~S}$ rRNA as illustrated in Table 3-5.

Table 3-5 Copy number of 16S rRNA in each bacterial genome (Větrovský \& Baldrian, 2013).

\begin{tabular}{|l|l|l|}
\hline Phylum & 16S rRNA/genome & $\begin{array}{l}\text { Mean 16S } \\
\text { rRNA/genome }\end{array}$ \\
\hline Acidobacteria & 1 & 1 \\
\hline Actinobacteria & $3.3 \pm 1.7$ & 3.3 \\
\hline Bacteroidetes & $3.5 \pm 1.5$ & 3.5 \\
\hline Firmicutes & $5.8 \pm 2.8$ & 5.8 \\
\hline Proteobacteria & $3.96 \pm 1.7$ & 4.0 \\
\hline
\end{tabular}

\subsubsection{High-throughput sequencing}

DNA libraries were prepared following the 16S Barcoding Kit (SQK-RAB204) protocol and used to generate the $16 \mathrm{~S}$ bacterial community profiles. Each library, consisting of uniquely barcoded time points, was run separately in a MIN 107 MinION $^{\mathrm{TM}}$ flowcell. The basecalled data from each run was used in the EPI2ME Fastq $16 \mathrm{~S}$ workflow, which generated BLAST data that was directly used for diversity and community analyses. The MinION $^{\mathrm{TM}}$, flowcell and kits were purchased from Oxford Nanopore Technologies, New York, USA, and the library preparation and sequencing were done according to the 
MinION 1D 16S Barcoding Kit (SQK-RAB204) protocol. PCR was used to amplify the DNA samples by using specific $16 \mathrm{~S}$ primers (27F and 1492R) with barcodes, and 5' tags that facilitate ligase-free attachment of Rapid Sequencing Adapters. The PCR products were cleaned, concentrated with Agencourt AMPure XP beads and quantified with the Qubit $^{\circledR}$ dsDNA HS Assay Kit using the Qubit 3.0 Fluorometer (Molecular Probes, Life Technology, Eugene, OR). The PCR products for each library were then pooled at the required ratio, a rapid sequencing adapter attached, and then introduced and attached to loading beads which were then loaded into the MinION flow cell. The MinION protocol uses software named MinKNOW which allowed for real-time basecall monitoring; each library sequencing run was stopped at $\sim 150,000$ reads to maintain consistent read depths for all soil sample libraries.

\subsection{DNA extraction, quantification and quality control}

Genomic DNA was extracted from sludge or soil and quantified according to the procedures provided in Section 3.7.1. The purity of the samples was measured with the NanoDrop, by assessing the 260/280 $\mathrm{nm}$ and $260 / 230 \mathrm{~nm}$ ratios, and congruence of concentrations with Qubit values. In addition, the genomic DNA was assessed using gel electrophoresis to ensure fragment sizes were between 3 and $10 \mathrm{Kbps}$ which is required for optimal MinION sequencing. The genomic DNA extracts were run on a $0.8 \%$ agarose gel at $7 \mathrm{~V} / \mathrm{cm}^{2}$. The agarose gel was prepared in TAE buffer. $6 \mathrm{X}$ gel loading dye and GeneRuler $1 \mathrm{~Kb}$ ready-to-use DNA Ladder from Thermo Fisher were used. 


\subsection{Metagenomic analysis}

The MinKNOW generated Fastq files were BLASTed against the NCBI 16S bacterial database using Oxford Nanopore's cloud-based analysis platform EPI2ME with the $16 \mathrm{~S}$ Fastq workflow. Each read was classified based on \% coverage and identity. Results were transcribed into excel datasheets where they were sorted into different taxonomy classifications (mainly phylum and genus levels) for every time point and each soil reactor. A 3\% sequence dissimilarity cut-off value, commonly used to estimate the species level of phylotype diversity, was universally applied. During the study, biodiversity indices were estimated for the top detected phyla with a relative abundance of $\geq 0.01 \%$. Alpha biodiversity (Shannon-Wiener index, richness, evenness) and Beta-Euclidean biodiversity were estimated and analyzed for bacteria at the phylum level. The relative abundance was estimated and compared for each soil reactor at both phylum and genus levels. The cutoff value for biodiversity analysis at the genus level was $\geq 1 \%$ relative abundance. Also, several interesting genera were discussed, including those with a relative abundance of $\leq$ $1 \%$. These genera were selected based on their function in the anaerobic digestion process

or the soil. Shannon-Wiener index, used to characterize the phyla diversity in a community, was determined by calculating the proportion of a phylum to the other phyla in a community, then multiplying it by the natural logarithm of this proportion. The resulting values are summed across the phyla and multiplied by -1 (Equation 3-1) (Magurran, 1988). Phyla evenness was calculated according to Equation 3-2 (Magurran, 1988; Mulder et al., 2004). Phyla evenness is between 0 and 1, with 1 being complete evenness. Phyla richness (N) is the total number of phyla in each sample or sample set after the cut-off. 
$H=-\sum_{i=1}^{N} p i \ln p i$

$E=\frac{H}{H \max }=H / \ln N$

\section{Equation 3-1}

\section{Equation 3-2}

Where $\quad H$ : Shannon-Wiener index

$N$ : total number of phyla

$p i$ : proportion of phylum $i$ to all other phyla

$E$ : phyla evenness

Beta-Euclidean diversity was calculated according to Equation 3-3 (Edgar, 2018).

Beta - Euclidean $=\operatorname{sqrt}\left(\sum_{1}^{N}(n k i-n k j)^{\wedge} 2\right)$

\section{Equation 3-3}

Where $\quad N$ : total number of phyla

$n k i$ : count of OTU $k$ in sample $i$

$n k j$ : count of OTU $k$ in sample $j$

\subsubsection{Live/Dead bacterial viability staining assay}

The distribution of live and dead bacterial cells in sludge and soil samples was investigated by staining with fluorescence dyes and observed with a Nikon Eclipse Ti-E Microscope equipped with Intensilight C-HGFI fluorescent illuminator and GFP-1, Cy3, and GFPHQ filters from Nikon, Montreal QC. The sludge and soil samples were stained as per manufacturer's instructions as described in the BacLight ${ }^{\circledR}$ Live/Dead viability assay kit L13152 literature (Molecular Probes, Eugene OR). The staining reagent was prepared by dissolving the SYTO 9 stain and propidium iodide in $5 \mathrm{~mL}$ of sterile deionized water. SYTO 9 stain is a green fluorescent nucleic acid stain that identifies the live cells and propidium iodide is a red fluorescent nucleic acid stain that penetrates the cells with damaged membranes and thereby indicates dead cells. This staining kit is designed for pure bacterial culture, therefore during this study, the sludge and soil samples were prewashed, 
before staining, to isolate the bacteria from sludge/soil particles and to remove a significant number of interfering compounds within the sample. The prewash was done by mixing 0.1 $\mathrm{mL}$ of sludge or $0.1 \mathrm{~g}$ of soil in a $0.9 \mathrm{~mL}$ of PBS solution with $0.1 \%$ Tween 80 , and the mixture was vortexed for $30 \mathrm{~s}$ at a high speed and settled for $30 \mathrm{~min}$, and the supernatant was used for the staining assay. The samples were prepared for Live/Dead analysis through transforming and mixing an equal volume of both the sample supernatant and the staining reagent into a $1.5 \mathrm{~mL}$ tube, vortex the mixture and incubate it in the dark for $15 \mathrm{~min}$. Then, a $17 \mu \mathrm{L}$ of the mixture was trapped between a slide and a $22 \mathrm{~mm}$ square coverslip. The samples were then assessed using a Nikon Eclipse Ti (Nikon, Montreal) inverted fluorescence microscope. Three random fields of views (FOV) were chosen on the slide where images were taken. Three images were taken at each FOV using 3 UV filters, GFP1, Cy-3 and GFPHQ to capture live, dead and a combination of live and dead microbial cells respectively. The three sets of images were assessed, and the most representative was chosen to report. The images were captured with the Q Imaging Retina Exi Fast 1394 camera (Nikon, Montreal QC) and the image analysis was performed with NIS-Elements software (Nikon, Montreal QC).

\subsubsection{Heterotrophic plate count}

The change in the viability of heterotrophic bacteria in the soil samples was investigated every 15 days using the spread plate method. Tryptic soy agar (TSA) was used as a growth media. TSA medium was prepared by dissolving $30 \mathrm{~g}$ of the powdered TSA in water, sterilized by autoclaving and letting it cool to approximately $50^{\circ} \mathrm{C}$ before using. A volume of $22 \mathrm{~mL}$ was then poured into sterile $100 \mathrm{~mm}$ diameter Petri plates and stored at $4^{\circ} \mathrm{C}$ for a maximum of one week before use. $0.1 \mathrm{~g}$ of soil sample was suspended in $0.9 \mathrm{~mL}$ PBS 
(with $0.1 \%$ Tween 80 ) and vortexed for $30 \mathrm{~s}$. Serial dilutions were made and three $0.1 \mathrm{~mL}$ replicates of 10E-04, 10E-05 and 10E-06 dilutions were inoculated onto TSA plates and incubated in the dark at $34 \pm 1^{\circ} \mathrm{C}$. Colony forming units (CFU) were calculated after $24 \mathrm{~h}$ of incubation.

\subsection{Chemical analyses}

All chemical analyses were conducted according to Standard Methods (APHA et al., 2017). The sludge or soil samples were taken from the reactors and three replications of each test were performed. The average values and standard deviations were calculated.

\subsubsection{Chemical oxygen demand}

Chemical oxygen demand (COD) was analyzed using the HACH Method 8000 from $\mathrm{HACH}$, London, ON. It was done by heating the sample for two hours with sulfuric acid and potassium dichromate, a strong oxidizing agent. All the oxidizable organic compounds react and reduce dichromate ions $\left(\mathrm{Cr}_{2} \mathrm{O}_{7}^{2-}\right)$ to green hexaaquachromium ions $\left(\mathrm{Cr}^{3+}\right)$. The amount of $\mathrm{Cr}^{6+}$ remaining is determined when the low concentration range of 3-150 mg/L is used, and when high concentration range of $20-1500 \mathrm{mg} / \mathrm{L}$ is used, the amount of $\mathrm{Cr}^{6+}$ produced is determined. COD is measured at a wavelength of $420 \mathrm{~nm}$ for the low range and $620 \mathrm{~nm}$ for the high range test kit.

\subsubsection{Total nitrogen}

Samples were analyzed for TN according to $\mathrm{HACH}$ method 10072, from $\mathrm{HACH}$, London, ON. persulfate digestion method and a HACH DR 2800 spectrophotometer were used to measure TN in the sample. All forms of nitrogen in the sample will be converted to nitrate 
by alkaline persulfate digestion according to this method. Nitrate reacts with chromotropic acid under strongly acidic conditions to form a yellow complex with an absorbance maximum near $420 \mathrm{~nm}$.

\subsubsection{Total phosphorus}

Samples were analyzed for TP according to HACH Method 10127, from HACH, London, ON. The analysis was done using molybdovanadate method with acid persulfate digestion and a HACH DR 2800 spectrophotometer. According to this method, organic and condensed phosphates are converted to reactive orthophosphate by heating with acid and persulfate. The measurement wavelength is $880 \mathrm{~nm}$.

\subsubsection{Total organic carbon}

TOC analysis was conducted using TOC-VCPH/CPN from Shimadzu and TOC-control V software. Each TOC measurement run included a blank and a set of standard solution concentrations used to construct a calibration curve. Three replicates were taken for each TOC measurement. Soil samples were prepared by suspending $1 \mathrm{~g}$ of soil in $10 \mathrm{~mL}$ of $\mathrm{CaCl}_{2}$ solution $(1: 10 \mathrm{w} / \mathrm{v}$ ratio, $0.01 \mathrm{M})$. The suspension was put in a shaker for $2 \mathrm{~h}$ then centrifuged at $1,800 \times \mathrm{g}$ for $12 \mathrm{~min}$. The supernatant was taken for the TOC analysis.

\subsection{5 $\mathrm{pH}$}

Soil samples were prepared for $\mathrm{pH}$ analysis by suspending $5 \mathrm{~g}$ of soil in $5 \mathrm{~mL}$ of water (1g: $1 \mathrm{~mL}$ ratio), and the suspension was vortexed for $30 \mathrm{~s}$ and taking the $\mathrm{pH}$ measurement using an Orion-Thermo Orion Star тм A326 pH/DO portable meter. The $\mathrm{pH}$ for sludge was measured directly. 


\subsubsection{Total solids and volatile solids for sludge}

The percentage of TS and VS were measured according to Part 2540 Standard Methods (APHA et al., 2017). TS were determined by drying $5 \mathrm{~mL}$ wastewater sludge suspension in the oven at $105^{\circ} \mathrm{C}$ for $24 \mathrm{~h}$, and the difference in the weight of the sample before and after drying was the TS per sample volume multiplied by 100 . VS was determined by placing the $\mathrm{TS}$ in the furnace at $550^{\circ} \mathrm{C}$ for $30 \mathrm{~min}$ and VS was calculated from the difference between the TS before and after the furnace and multiplying by 100 .

\subsubsection{Total solids and moisture content for soil}

The TS and MC were determined by drying $5 \mathrm{~g}$ of the soil sample in the oven at $105^{\circ} \mathrm{C}$ for $24 \mathrm{~h}$. The TS\% was represented by the sample weight after drying divided by sample weight before drying and multiplying by 100 . $\mathrm{MC}$ was calculated by taking the difference in the sample weight before and after drying, dividing it by the weight of the dried sample and multiplying by 100 . MC was maintained at $45 \%$ for all soil reactors by taking three measurements every 2-3 d. If the moisture was to be increased, sterile Milli-Q water was applied to the reactor.

\subsubsection{Cation exchange capacity}

CEC is an essential property of soil that determines the concentration of negatively charged sites on soil colloids that can adsorb exchangeable cations. The soil was analyzed for CEC at the beginning of each experiment using a standard method used by the Natural Resources Conservation Service (Rebecca, 2004). The analysis method involved using $1 \mathrm{M}$ ammonium acetate $\left(\mathrm{NH}_{4} \mathrm{OAc}\right)$ at $\mathrm{pH} 7$ (neutral $\mathrm{NH}_{4} \mathrm{OAc}$ ). 


\subsection{Experimental design}

\subsubsection{Anaerobic digestion of sludge}

Anaerobic digestion of wastewater sludge was conducted using batch mesophilic biochemical methane potential (BMP) tests in $125 \mathrm{~mL}$ Erlenmeyer flasks sealed with rubber stoppers. The BMP assays were done by mixing three types of sludge at a specific ratio. Nitrogen sparging was applied to the batch reactors when the three types of sludge were mixed, to prevent exposure to oxygen. The flasks were sealed after adding equal amounts of $\mathrm{NaHCO}_{3}$ and $\mathrm{KHCO}_{3}$ to achieve alkalinity of $4000 \mathrm{mg} / \mathrm{L}\left(\right.$ as $\left.\mathrm{CaCO}_{3}\right)$. The BMP reactors were kept in the dark at $34 \pm 1{ }^{\circ} \mathrm{C}$ on the shaker at $100 \mathrm{rpm}$ in a temperature-

controlled incubator. Several periodic sample analyses were done, including total and volatile solids, COD, Live/Dead cells and DNA extraction throughout the digestion period, which was $40 \mathrm{~d}$.

\subsubsection{Chemical conditioning}

Chemical conditioning involved fast mixing for 1 min and slow mixing for $30 \mathrm{~min}$. The $\mathrm{pH}$ was recorded after chemical addition, and for ferric chloride and alum, was adjusted to the desired value using $1 \mathrm{M} \mathrm{NaOH}$ (sodium hydroxide). Chemical doses were chosen based on preliminary coagulation-flocculation experiments that were carried out to find the optimum chemical dose. This was done using a jar tester, where four different doses of each studied chemical were examined (Table 3-6). The evaluation of dewaterability was based on the capillary suction time test (CST) of the conditioned sludge and the turbidity of the supernatant. Three measurements of CST were taken for the conditioned sludge at the end of the experiments by placing $10 \mathrm{~mL}$ aliquots of the conditioned sludge in the CST 
reservoirs, Triton Electronics type 31, Multi-CST device. The turbidity of the supernatant was measured after settling for 30 min using the HACH 2100AN turbidimeter. Thus, the optimal dose was chosen based on the results obtained (Table 3-6) and from literature (Metcalf \& Eddy, 2003; Krishnamurthy \& Viraraghavan, 2005; Murray \& Ormeci, 2009). Table 3-6 Coagulation-flocculation experiment.

\begin{tabular}{|c|l|l|l|l|}
\hline Chemical & $\begin{array}{c}\text { Chemical/sludge } \\
\mathbf{( g / g )}\end{array}$ & \multicolumn{1}{|c|}{$\mathbf{p H}$} & \multicolumn{1}{|c|}{ CST (s) } & \multicolumn{1}{c|}{$\begin{array}{c}\text { Turbidity } \\
\mathbf{( N T U )}\end{array}$} \\
\hline \multirow{4}{*}{ FeCl $3_{3}$} & 0.4 & $6.7 \pm 0.1$ & $331 \pm 13$ & $272 \pm 7$ \\
\cline { 2 - 5 } & 0.8 & $6.21 \pm 0.02$ & $119.1 \pm 7$ & $198 \pm 4$ \\
\cline { 2 - 5 } & 0.1 & $6.0 \pm 0.03$ & $41.8 \pm 5$ & $64 \pm 2$ \\
\cline { 2 - 5 } Alum & 1.3 & $5.6 \pm 0.1$ & $97 \pm 5$ & $66 \pm 2$ \\
\hline \multirow{5}{*}{ Polymer } & 0.18 & $6.14 \pm 0.13$ & $436.3 \pm 16$ & $280 \pm 5$ \\
\cline { 2 - 5 } & 0.36 & $4.98 \pm 0.08$ & $194.2 \pm 10$ & $170 \pm 4$ \\
\cline { 2 - 5 } & 0.7 & $3.2 \pm 0.08$ & $64 \pm 5$ & $62 \pm 3$ \\
\cline { 2 - 5 } & 1.4 & $1.8 \pm 0.03$ & $120 \pm 11$ & $73 \pm 3$ \\
\cline { 2 - 5 } & 0.0026 & $7.5 \pm 0.1$ & $191.2 \pm 14$ & $124 \pm 8$ \\
\cline { 2 - 5 } & 0.0052 & $7.5 \pm 0.1$ & $17.1 \pm 4$ & $60 \pm 4$ \\
\cline { 2 - 5 } & 0.01 & $7.5 \pm 0.1$ & $11.8 \pm 2$ & $52 \pm 3$ \\
\hline
\end{tabular}

Data ( $\mathrm{pH}, \mathrm{CST}$, and turbidity) are the average of three replicate measurements \pm standard deviation.

\subsubsection{Lime stabilization of sludge}

Lime stabilization was performed according to methods described by Metcalf \& Eddy (2003) and Clint et al. (2007), that involved adding lime slurry to the sludge while stirring it continuously, and measuring the $\mathrm{pH}$ until a $\mathrm{pH}$ of 12 or higher had been reached and maintained for two hours. The lime slurry was prepared by mixing powdered quicklime with water at a concentration of $150 \mathrm{mg} / \mathrm{mL}(15 \%)$.

\subsubsection{Land application of sludge}

Glass beakers were filled with dry soil and used as soil reactors. The sludge/soil ratio was $0.01 \mathrm{~g} / \mathrm{g}$ (dry weight), and it was selected based on the biosolids land application guidelines at a rate of 8 ton/ha every 5 years (OME \& OMAFRA, 1996). Other studies were also 
considered in choosing the sludge application ratio (Colman et al., 2013; Whitley et al., 2013). During this research, sludge was applied to the soil in liquid form after treatment. The soil reactors were incubated with day/night cycles of $8 / 16 \mathrm{~h}$ at $22 \pm 1{ }^{\circ} \mathrm{C}$. Soil reactors were started with $60 \%$ moisture content and after were maintained at $45 \%$ for all soil reactors by monitoring the soil every three days. Chemical and biological analyses were conducted every 15 days to examine the soil activity during the incubation time for 105 days.

\subsection{Statistical methods}

All results are reported as the average of three replicates \pm standard deviation. Comparisons and significant differences between different soil or sludge reactors over time were determined using a one-way analysis of variation (ANOVA) at 95\% confidence level. Significant differences are represented by $p$-values $<0.05$, and if there are significant differences between two reactors, the Post Hoc Tukey test was used to separate the means

further, using the same significance level (Currell \& Dowman, 2005). In addition, the coefficient of variance $(\mathrm{CV})$ was estimated for each data set. Significant differences are those with a $\mathrm{CV}>0.05$ (i.e. level of confidence $\geq 95 \%$ ). 


\section{References}

APHA, A. P., American water works Association, A., \& Water environment federation, W. (2017). Standard methods for the examination of water and wastewater (23 ed.). (E. Rice, R. Baird, \& A. Eaton, Eds.) Washington, DC, USA: APHA, American Public Health Association; American water works Association, AWWA; Water environment federation, WEF.

Baalousha, M., Stolpe, B., \& Lead, J. (2011). Flow field-flow fractionation for the analysis and characterization of natural colloids and manufactured nanoparticles in environmental systems: a critical review. Journal of Chromatography A, 1218(27), 4078-4103.

Beermann, B., Carrillo-Nava, E., Scheffer, A., Buscher, W., Jawalekar, A. M., Seela, F., \& Hinz, H.-J. (2007). Association temperature governs structure and apparent thermodynamics of DNA-gold nanoparticles. Biophysical Chemistry, 126(1-3), 124-131.

City of Ottawa, C. o. (2014). Let's explore...Wastewater treatment at the Robert O. Pickard Environmental Centre (ROPEC). Ottawa, Canada: City of Ottawa annual report.

Clint, W., Chen, W.-Y., Shammas, N. K., \& Wang, L. K. (2007). Lime Stabilization. In N. K. Shammas, \& L. K. Wang (Eds.), Handbook of Environmental Engineering: Biosolids Treatment Processes (pp. 207-241). Totowa, N: The Humana Press Inc.

Colman, B. P., Arnaout, C. L., Anciaux, S., Gunsch, C. K., Hochella Jr, M. F., Kim, B., . . al., e. (2013). Low Concentrations of Silver Nanoparticles in Biosolids Cause Adverse Ecosystem Responses under Realistic Field Scenario. PLOS One, 8(2), e57189.

Currell, G., \& Dowman, A. (2005). Essential mathematics and statistics for science. Chichester;Hoboken, NJ: Wiley \& Sons.

Edgar, R. (2018). Usearch V11, Euclidean distance metric. Retrieved from drive5 Bioinformatics software and services: https://drive5.com/usearch/manual/euclidean_distance.html

Espinosa-Ortiz, E. J., \& Vaca-Mier, M. (2013). Physical and chemical analysis of nanoparticles. In P. Lens, J. Virkutyte, V. Jegatheesan, S. Al-Abed, \& S.-H. Kim (Eds.), Nanotechnology for water and wastewater treatment (pp. 83-125). London: IWA Publishing . 
Fierer, N., Jackson, J. A., Vilgalys, R., \& Jackson, R. B. (2005). Assessment of soil microbial community structure by use of taxon-specific quantitative PCR assays. Appl. Environ. Microbiol., 71(7), 4117-4120.

Gregorich, E. G., \& Carter, M. R. (2007). Soil sampling and methods of analysis (2nd ed.). CRC Press.

Houba, V. J., Temminghoff, E. J., Gaikhorst, G. A., \& Vark, W. V. (2000). Soil analysis procedures using 0.01 M calcium chloride as extraction reagent. Communications in Soil Science and Plant Analysis, 31(9-10), 1299-1396.

Krishnamurthy, S., \& Viraraghavan, T. (2005). Chemical conditioning for dewatering municipal wastewater sludges. Energy Sources, 1-2(27), 113-122.

Laborda, F., Bolea, E., \& Jiménez-Lamana, J. (2014). Single particle inductively coupled plasma mass spectrometry: a powerful tool for nanoanalysis. Analytical Chemistry, 86(5), 22702278.

Magurran, A. E. (1988). Ecological diversity and its measurement. Princeton university press.

Mavrocordatos, D., Pronk, W., \& Boiler, M. (2004). Analysis of environmental particles by atomic force microscopy, scanning and transmission electron microscopy. Water Science and Technology, 50(12), 9-18.

Metcalf, L., \& Eddy, H. P. (2003). Waste Water Engineering: Treatment and Reuse (4 ed.). New York: McGraw-Hill.

Morales, S. E., \& Holben, W. E. (2009). Empirical testing of 16S rRNA gene PCR primer pairs reveals variance in target specificity and efficacy not suggested by in silico analysis. Applied and Environmental Microbiology, 75(9), 2677-2683.

Mulder, C., Bazeley-White, E., D., P.G., H. A., Scherer-Lorenzen, M., \& Schmid, B. (2004). Species evenness and productivity in experimental plant communities. Oikos, 107(1), 5063.

Murray, A., \& Ormeci, B. (2009). Determination of Optimum Polymer Dose for Single and Dual Polymer Conditioning of Wastewater Sludge. Journal of Residuals Science \& Technology, $6(2)$. 
OME, O. M., \& OMAFRA, O. M. (1996). Guidlines for The Utilization of Biosolid and Other Waste on Agricultural lands. Ontario, Canada: Ontario.

Pace, H. E., Rogers, N. J., Jarolimek, C., Coleman, V. A., Higgins, C. P., \& Ranville, J. F. (2011). Determining transport efficiency for the purpose of counting and sizing nanoparticles via single particle inductively coupled plasma mass spectrometry. Analytical Chemistry, 83(24), 9361-9369.

Pfeiffer, S., Milica, P., Birgit, M., Kathrin, L., Evelyn, H., Paul, L., . . Angela, S. (2014). Improved group-specific primers based on the full SILVA 16S rRNA gene reference database. Environmental Microbiology, 16(8), 2389-2407.

Rebecca, B. (2004). Soil survey laboratory method manual. Soil Survey Laboratory Investigations Report.

Shakya, M., Christopher, Q., James H., C., Zamin K., Y., Christopher W., S., \& Mircea, P. (2013). Comparative metagenomic and rRNA microbial diversity characterization using archaeal and bacterial synthetic communities. Environmental Microbiology, 15(6), 1882-1899.

Svec, D., Ales, T., Vendula, N., Michael W., P., \& Mikael, K. (2015). How good is a PCR efficiency estimate: Recommendations for precise and robust qPCR efficiency assessments. Biomolecular Detection and Quantification, 3, 9-16.

Thomas, R. (2013). Practical Guide to ICP-MS (3rd ed.). New York: Marcel Dekker, Inc.

Větrovský, T., \& Baldrian, P. (2013). The variability of the 16S rRNA gene in bacterial genomes and its consequences for bacterial community analyses. PloS One, 8(2), 57923.

Whitley, A. R., Levard, C., Oostveen, E., Bertsch, P. M., Matocha, C. J., Kammer, F. v., \& Unrine, J. M. (2013). Behavior of Ag nanoparticles in soil: effects of particle surface coating, aging and sewage sludge amendment. Environmental Pollution, 182, 141-149. 


\title{
Chapter 4: Impact of AgNPs and CuONPs on The Anaerobic Digestion of Sludge and The Structure of Bacterial Community
}

\begin{abstract}
Commercial applications of silver nanoparticles (AgNPs) and copper oxide nanoparticles (CuONPs) have ultimately led to their release into the environment, and wastewater sludge has been identified as one of the major paths for the release of nanoparticles. Thus, concerns regarding the fate and toxicity of AgNPs and CuONPs have been growing due to their antimicrobial activity and toxicity. This study investigates the impact of different concentrations of AgNPs and CuONPs on bacterial communities and process performance during anaerobic digestion of sludge. Anaerobic digestion was carried out by conducting batch mesophilic biochemical methane potential (BMP) tests to treat mixed primary and secondary sludge. There were seven BMP reactors; the control (no nanoparticles), three reactors containing 2, 10, and $30 \mathrm{mg} \mathrm{AgNPs/g} \mathrm{TS}$, and three reactors containing 2, 10, and $30 \mathrm{mg} \mathrm{CuONPs} / \mathrm{g}$ TS. All reactors were incubated at $35 \pm 1{ }^{\circ} \mathrm{C}$ in anaerobic conditions and sampled every day for biogas generation and 6 times for chemical oxygen demand (COD), $\mathrm{pH}$, total solids (TS), volatile solids (VS), Live/dead cells, and DNA concentration throughout $37 \mathrm{~d}$ period. Bacterial abundance and diversity were investigated using highthroughput sequencing. The metagenomic analyses identified the same three dominant phyla in each BMP reactor: Proteobacteria, Firmicutes, and Bacteroidetes, all of which represented $>98 \%$ of the total observed phyla operational taxonomic units (OUTs). The results also showed that AgNPs and CuONPs at 2, 10 and $30 \mathrm{mg} / \mathrm{g}$ TS sludge had no significant impact on the overall bacterial community structure at phylum and genus levels
\end{abstract}


over time. AgNPs and CuONPs also did not significantly impact the biogas generation over time, except for sludge reactor that had higher concentration of CuONPs (30 mg NPs/g TS), which showed significant reduction in biogas generation over time.

Keywords: silver nanoparticles, copper oxide nanoparticles, sludge, anaerobic digestion, biogas generation, bacterial communities, phyla, genera

\subsection{Introduction}

Anaerobic digestion is a widely used and environmentally friendly process to treat wastewater sludge by converting biodegradable organic matter into energy (Metcalf \& Eddy, 2003; Taricska et al., 2007). The process involves a series of complex microbial reactions that stabilize sludge by removing organic matter and pathogens, and also produce biogas. Successful anaerobic digestion depends on the characteristics of different microbial communities inside the reactor, as well as their critical functions. Many factors can potentially affect the performance of anaerobic digestion, including the presence of toxic elements. Nanoparticle contamination and the impact on the anaerobic process are of particular interest in today's research.

Extensive use of nanoparticles in various industrial activities has led to their presence in the environment, and this can potentially have negative impacts. Both AgNPs and CuONPs are widely known for their antimicrobial activity and have been commonly used as preservatives in many consumer products, as well as for medicinal purposes (Lansdown, 2004; Babu et al., 2015; Battez et al., 2010; US Research Nanomaterials, 2017). The broad usage of AgNPs and CuONPs has resulted in their release into the waste stream, mainly 
through wastewater (Gottschalk et al., 2009; Keller et al., 2013). Several recent studies found that the majority of nanoparticles that enter wastewater are retained in wastewater sludge (Shafer et al., 1998, Kaegi et al., 2011, Li et al., 2016).

One of the main uses of AgNPs and CuONPs is to inhibit microbial growth, and there is a significant concern that the presence of these nanoparticles could adversely affect the efficiency of biological systems. Several studies in the literature show the toxic impact of AgNPs and CuONPs on different organisms, including humans (Jo et al., 2012; Jang et al., 2016; Juling et al., 2017). Nanoparticles have also been examined and shown to affect different wastewater treatment processes. For example, Liang et al. (2010) investigated the impact of AgNPs on a modified Ludzack-Ettinger activated sludge system and observed a $46.5 \%$ decrease in denitrification when the system was exposed to an acute load of AgNPs (concentration of $0.75 \mathrm{mg} / \mathrm{L}$ for $12 \mathrm{~h}$ ). Also, Sun et al. (2013) showed that the presence of AgNPs at $1 \mathrm{mg} / \mathrm{L}$ for $24 \mathrm{~h}$ during the activated sludge process impacted some microbial species but did not decrease heterotrophic plate count (HPC) before settling. After settling, HPC decreased by one log with no observed changes in microbial diversity. Another study by Qiu et al. (2016) found no significant effect of AgNPs on organic matter removal, nitrification and denitrification during sequencing batch reactor; however, increased the production of extracellular polymeric substances due to cell self-protection was observed. As well, Zhang et al. (2017) studied the impact of CuONPs on simulated waste-activated sludge system, and reported that the presence of 1,10 , and $50 \mathrm{mg} \mathrm{CuONPs} / \mathrm{L}$ decreased the COD removal from $78.7 \%$ to $77.0 \%, 52.1 \%$, and $39.2 \%$, respectively, and increased effluent ammonium $\left(\mathrm{NH}_{4}-\mathrm{N}\right)$ concentration from $14.9 \mathrm{mg} / \mathrm{L}$ to $18.0,25.1$, and $30.8 \mathrm{mg} / \mathrm{L}$, respectively. 
Previous studies that investigated the behavior and impact of AgNPs and CuONPs during anaerobic digestion suggested a decrease in biological activity (Wang et al., 2016; LunadelRisco et al., 2011; Rasool \& Lee, 2016). For example, Rasool and Lee (2016) observed decreases of 51.8 and $33.6 \%$ in sulfate and COD removal efficiencies, respectively at 50 to $200 \mathrm{mg} / \mathrm{L}$ concentration of AgNPs and found no impact on sulfate and COD removal efficiencies when using $\leq 10 \mathrm{mg} / \mathrm{L}$ AgNPs. Another study by Gonzalez-Estrella et al. (2013) examined the inhibitory impact of several different nanoparticles on methanogenic activity. They found that CuONPs inhibited the acetoclastic methanogens $($ IC50 $=223$ mg/L) with no significant impact on hydrogen utilizing methanogens, while AgNPs also had no significant impact. Similar findings by Ünşar et al. (2016) showed a 5.8 to $84 \%$ increase in inhibition of anaerobic digestion when using 5-1000 mg/g TS of CuONPs, and only a $12.1 \%$ decrease in methane production due to AgNPs when using the same concentration range.

Although several studies stated that nanoparticles could lower the volume of methane collected (Wang et al., 2016; Luna-delRisco et al., 2011; Ünşar et al., 2016), the impact on microbial population and diversity remains unclear, and whether this impact is universal or applicable to specific situations is still largely unexplored. For example, several factors could contribute to the impact of nanoparticles, including the origin of nanoparticles (i.e., pristine or modified), their concentration, sludge characteristics, and environmental factors (e.g., temperature, oxygen concentration, $\mathrm{pH}$ ).

This study investigated the impact of AgNPs and CuONPs on anaerobic digestion of sludge and the structure of the bacterial community. A comparative community analysis was performed to examine the bacterial population and diversity of a control sample and six 
other BMP reactors with different concentrations of AgNPs and CuONPs. The objectives of the study were to examine the shift in bacterial communities during anaerobic sludge digestion and identify the dominant bacteria involved. This was achieved by operating seven BMP reactors for 37 days under controlled mesophilic environmental conditions where periodic chemical and biological assessments were performed, including metagenomic analysis. Metagenomic data were generated by high-throughput sequencing of the 16S rRNA gene using the MinION to explore bacterial populations and diversity. Bacterial community composition was analyzed and studied by comparing the sludge reactors with the nanoparticles to the control reactor and each other. Analysis of several bacteria of interest, including their potential functions during the process was also discussed.

\subsection{Materials and methods}

\subsubsection{Silver and copper oxide nanoparticles}

The AgNPs and CuONPs used in this study were purchased as a suspension in nanopure water. The stock suspension of pristine AgNPs in water with a purity of $99.9 \%$ was acquired from nanoComposix Inc. (San Diego, CA, USA). The AgNPs particle size distribution was examined and confirmed by transmission electron microscopy (TEM) analysis, and the AgNPs suspension stock solution was maintained at $4 \pm 0.2^{\circ} \mathrm{C}$. The stock solution of CuONPs with $99.95 \%$ purity was purchased from US Research Nanomaterials Inc. (Houston, USA) and maintained at $4 \pm 0.2^{\circ} \mathrm{C}$. Both nanoparticles suspensions were stable and insoluble in nanopure water. The properties of both nanoparticles are shown in Table 4-1. 
Table 4-1 AgNPs and CuONPs characteristics.

\begin{tabular}{|l|l|l|}
\hline Characteristics & AgNPs & CuONPs \\
\hline Diameter (TEM) & $23.1 \pm 6.9 \mathrm{~nm}$ & $25-55 \mathrm{~nm}$ \\
\hline pH of the solution & 6.3 & $7-9$ \\
\hline Morphology & Nearly spherical & Nearly spherical \\
\hline Solvent & Milli-Q water & Milli-Q water \\
\hline Particle surface coating & $\begin{array}{l}\text { Polyvinylpyrrolidone } \\
\text { (PVP) }\end{array}$ & None \\
\hline
\end{tabular}

\subsubsection{Wastewater sludge samples}

Three different types of wastewater sludge were collected at the Robert O. Pickard Environmental Center (ROPEC) wastewater treatment plant in Ottawa (ON, Canada). Primary sludge was obtained from the effluent line of the primary clarifier, and thickened waste activated sludge (tWAS) was collected from the effluent line of the thickener centrifuge that receives waste activated sludge. Anaerobically digested sludge was collected from the effluent line of the anaerobic digester and was used as an inoculum. ROPEC is the main wastewater treatment facility in Ottawa and processes approximately 390 million litres of wastewater per day. ROPEC has preliminary treatment, primary treatment, and uses aerobic activated sludge process for biological treatment. Ferric chloride is added in the aeration tank to remove phosphorus, and mixture of primary and thickened secondary sludge is then treated by anaerobic digestion. The wastewater sludge characteristics are given in Table 4-2. All wastewater sludge samples were collected in autoclave sterilized containers and were used either on the day of collection or stored at $4^{\circ} \mathrm{C}$ for a maximum of four days before using. 
Table 4-2 Characterization of the three types of raw wastewater sludge.

\begin{tabular}{|l|l|l|l|}
\hline \multirow{2}{*}{ Parameters } & \multicolumn{2}{|l|}{ Substrate } & \multirow{2}{*}{$\begin{array}{l}\text { Anaerobically digested } \\
\text { sludge (inoculum) }\end{array}$} \\
\cline { 2 - 3 } & Primary sludge & tWAS & $7.3 \pm 0.02$ \\
\hline $\mathrm{pH}$ & $7.6 \pm 0.1$ & $7.0 \pm 0.1$ & $19.0 \pm 0.2$ \\
\hline $\mathrm{TS}(\mathrm{g} / \mathrm{kg})$ & $42.7 \pm 7.2$ & $52.7 \pm 1.6$ & $15.6 \pm 0.1$ \\
\hline VS $(\mathrm{g} / \mathrm{kg})$ & $28.7 \pm 3.5$ & $38.6 \pm 1.5$ & $19.8 \pm 1.1$ \\
\hline $\begin{array}{l}\text { Total chemical oxygen } \\
\text { demand (tCOD) }(\mathrm{g} / \mathrm{kg})\end{array}$ & $52.0 \pm 8.3$ & $73.9 \pm 2.1$ & $8.4 \pm 0.2$ \\
\hline $\begin{array}{l}\text { Soluble chemical oxygen } \\
\text { demand (sCOD) }(\mathrm{g} / \mathrm{kg})\end{array}$ & $2.0 \pm 1.1$ & $8.2 \pm 0.1$ & \\
\hline
\end{tabular}

Values are the mean of three replicate measurements \pm standard deviation.

\subsubsection{Experimental design}

Anaerobic digestion of the sludge was conducted using batch BMP tests conducted in 125 $\mathrm{mL}$ Erlenmeyer flasks sealed with rubber stoppers. There were seven main BMP reactors, of which one reactor was the control, which had no nanoparticles, three reactors had different concentrations of AgNPs (2, 10, and $30 \mathrm{mg} \mathrm{AgNPs/g} \mathrm{TS})$, and three reactors had different concentrations of CuONPs $(2,10$, and $30 \mathrm{mg} \mathrm{CuONPs/g} \mathrm{TS})$. The BMP tests were done in three replicates. The lower nanoparticle concentration used represents the higher range of the literature predicted or environmental occurrence concentrations in the wastewater sludge (CCME, 2010; EPA, 2009; Blaser et al., 2008; Gottschalk et al., 2013), while the medium and high concentrations were investigated to be able to observe the potential impact of accumulated nanoparticles, and were chosen based on previous studies as well (Wang \& Chen, 2016). Table 4-3 shows the properties of each reactor. Each Erlenmeyer flask had $15 \mathrm{~mL}$ of anaerobically digested sludge (inoculum), $35 \mathrm{~mL}$ of primary sludge and $35 \mathrm{~mL}$ of tWAS, to make up a final sludge mixture volume of $85 \mathrm{~mL}$, and equal amounts of $\mathrm{NaHCO}_{3}$ and $\mathrm{KHCO}_{3}$ were added to achieve alkalinity of 4000-5000 
$\mathrm{mg} / \mathrm{L}$ (as $\mathrm{CaCO}_{3}$ ). Then, increasing concentrations of AgNPs and CuONPs were added to the BMP reactors (except the control), as described in Table 4-3. The BMP reactors were mixed continuously with nitrogen sparging during the preparation process to provide effective mixing and prevent exposure to air. In addition, to achieve a substrate to inoculum ratio $(\mathrm{S} / \mathrm{I})$ of 1.5 , the anaerobically digested sludge was used as inoculum after being centrifuged at $1800 \mathrm{rpm}$ for $10 \mathrm{~min}$ to concentrate it and increase its VS\% to acquire the $\mathrm{S} / \mathrm{I}$ of 1.5. The reactors were kept in darkness at $34 \pm 1^{\circ} \mathrm{C}$ on the shaker at $100 \mathrm{rpm}$ in a temperature-controlled incubator for 37 days. Several sludge characteristics and microbiological tests were carried out periodically, including biogas collection that was measured by inserting a syringe connected to a manometer, TS, VS, COD (both total and soluble), Live/Dead bacterial viability staining, and DNA extractions throughout experiments time. 
Table 4-3 BMP reactors characterizations and conditions.

\begin{tabular}{|c|c|c|c|c|c|}
\hline Reactors & $\begin{array}{c}\text { NPs } \\
\text { concentration } \\
\text { (mg NPs/g TS } \\
\text { of sludge) }\end{array}$ & $\begin{array}{l}\text { Sludge initially fed } \\
\text { to the reactor }\end{array}$ & $\begin{array}{c}\text { Reactor } \\
\text { volume } \\
(\mathrm{mL})\end{array}$ & $\begin{array}{c}\text { Reactor } \\
\text { temperature } \\
\left({ }^{\circ} \mathrm{C}\right)\end{array}$ & $\begin{array}{c}\text { Duration of } \\
\text { experiments } \\
\text { (days) }\end{array}$ \\
\hline Control & Zero & \multirow{7}{*}{$\begin{array}{c}\text { Waste activated } \\
\text { sludge }(40 \%)+ \\
\text { primary sludge }(40 \%) \\
+ \text { anaerobically } \\
\text { digested sludge }(20 \%)\end{array}$} & \multirow[t]{7}{*}{85} & \multirow[t]{7}{*}{$34 \pm 1$} & \multirow[t]{7}{*}{37} \\
\hline $\begin{array}{l}\text { Reactor A } \\
\text { (AgNPs) }\end{array}$ & 2 & & & & \\
\hline $\begin{array}{l}\text { Reactor B } \\
\text { (AgNPs) }\end{array}$ & 10 & & & & \\
\hline $\begin{array}{l}\text { Reactor C } \\
\text { (AgNPs) }\end{array}$ & 30 & & & & \\
\hline $\begin{array}{l}\text { Reactor D } \\
\text { (CuONPs) }\end{array}$ & 2 & & & & \\
\hline $\begin{array}{l}\text { Reactor E } \\
\text { (CuONPs) }\end{array}$ & 10 & & & & \\
\hline $\begin{array}{l}\text { Reactor F } \\
(\mathrm{CuONPs})\end{array}$ & 30 & & & & \\
\hline
\end{tabular}

\subsubsection{Chemical analyses}

The individual sludge types were initially characterized followed by chemical analysis of each reactor at $0,4,9,13,17,26$, and 37 days. Three replicates were done for each chemical analysis and their means and standard deviations are reported. All chemical analyses were carried out according to Standard Methods (APHA et al., 2017) and conducted by withdrawing a small volume of sludge from each reactor at each time point. COD measurements were also done according to Standard Methods (APHA et al., 2017), using the HACH Method 8000, 2007. COD was measured at a wavelength of $620 \mathrm{~nm}$ (high range) with $\mathrm{HACH}$ DR2800 portable spectrophotometer. Soluble COD measurements were performed after centrifugation of the sludge samples at $1800 \mathrm{rpm}$ for $20 \mathrm{~min}$, then filtering the supernatant through a $0.45 \mu \mathrm{m}$ syringe filter (Glassco Laboratory Equipment). TS and VS were determined based on Standard Methods, Part 2540 (APHA et al., 2017). The $\mathrm{pH}$ was monitored by taking measurements using an Orion-Thermo Orion Star TM A326 pH/DO portable meter. 


\subsubsection{Biological analyses}

Biological analyses were carried out on day $0,4,9,17,26$ and 37, as a result there were six time point measurements for each reactor over the duration of the experiments.

\subsubsection{Live/Dead bacterial viability staining assay}

Live/Dead bacterial viability staining was used to distinguish between viable and nonviable microbial cells within the microbial population. Sludge samples were stained as per instructions described in the BacLight ${ }^{\circledR}$ Live/Dead viability assay kit L13152 literature (Molecular Probes, Eugene OR). The staining reagent was prepared by dissolving the SYTO 9 and propidium iodide stains in $5 \mathrm{~mL}$ of sterile deionized water. The sludge samples were prepared for staining by mixing $0.1 \mathrm{~mL}$ of sludge with $0.9 \mathrm{~mL}$ of phosphate buffered saline (PBS) and vortexing for $30 \mathrm{~s}$. The mixture was left to settle for $1 \mathrm{~h}$, and the supernatant was used for staining. An equal amount of both the supernatant and the staining reagent were aliquoted into a $1.5 \mathrm{~mL}$ tube, mixed using a vortex and incubated at room temperature in the dark for $15 \mathrm{~min}$. After the incubation, $17 \mu \mathrm{L}$ of the mixture was trapped between a slide and a $22 \mathrm{~mm}$ square coverslip; the samples were then assessed using a Nikon Eclipse Ti (Nikon, Montreal) inverted fluorescence microscope. Three tests were done for each sludge sample, and three random fields of views (FOV) were chosen on the slide where images were taken. Three images were taken at each FOV using 3 UV filters, GFP-1, Cy-3 and GFPHQ to capture live, dead, and a combination of live and dead cells, respectively. The three sets of images were assessed, and the most representative was chosen to report. The images were captured with the Q-Imaging Retina Exi Fast 1394 
camera (Nikon, Montreal QC) and the image analysis was performed with NIS-Elements software (Nikon, Montreal QC).

\subsubsection{DNA extraction and quantification}

Genomic DNA was extracted using DNeasy PowerSoil DNA Isolation Kit from MO BIO Laboratories Inc. (QIAGEN Online Shop). To help reduce DNA extraction and downstream qPCR inhibitors and remove extracellular DNA all sludge samples were prewashed according to the procedure described by Kibbee and Ormeci (2017) before the extraction. The prewash step was performed by centrifuging a $0.5 \mathrm{~mL}$ of the sludge for 5 min at $8000 \times g$, discarding the supernatant, adding $1 \mathrm{~mL}$ of PBS to the pellet, vortexing for $15 \mathrm{~s}$, centrifuge for $5 \mathrm{~min}$ at $8000 \times g$, and discarding the supernatant, then the remaining pellet is ready for DNA extraction. Three replicate DNA extractions were done for each sludge sample according to manufacturers' instruction, with the exception of the cell lysis and inhibition removal steps where the incubation times were doubled from five to ten minutes. The concentration of extracted DNA was measured using the Qubit ${ }^{\circledR}$ dsDNA HS Assay Kits with the Qubit 3.0 Fluorometer from Molecular Probes (Life Technology, Eugene, OR). The DNA purity was assessed by measuring the absorbance ratios at, 260/280 and 260/230 nm using the NanoDrop 1000 Spectrophotometer (Thermo Scientific, Wilmington, USA). The genomic DNA was also assessed using gel electrophoresis to ensure fragment sizes were between 3 and $10 \mathrm{Kbps}$, as required for optimal MinION sequencing. Genomic DNA extracts were run on a $0.8 \%$ agarose gel at $7 \mathrm{~V} / \mathrm{cm}^{2}$; a TAE buffer was used to prepare the agarose gel. $6 \mathrm{X}$ gel loading dye and GeneRuler $1 \mathrm{~Kb}$ ready- 
to-use DNA Ladder from Thermo Fisher were used to visualize the genomic DNA size. The DNA samples were stored at $-20^{\circ} \mathrm{C}$ until use.

\subsubsection{Bacterial population analysis}

Bacterial diversity and population profiles of the sludge samples were analyzed using the $16 \mathrm{~S}$ rRNA BLAST results generated from the MinION sequencing runs and EPI2ME workflows.

\subsection{High-throughput sequencing}

In brief, seven genomic DNA libraries, prepared according to the MinION 1D 16S Barcoding Kit (SQK-RAB204) protocol, were used to generate the $16 \mathrm{~S}$ bacterial community profiles. Each library consisted of 6 uniquely barcoded time points, run separately in a MIN 107 MinION $^{\mathrm{TM}}$ flowcell. The basecalled data from each run was then used in the EPI2ME Fastq 16S workflow. This workflow generated BLAST data which was used directly for the diversity and community analyses. The MinION ${ }^{\mathrm{TM}}$, flowcell, and kits were purchased from Oxford Nanopore Technologies, New York, USA, and the library preparation and sequencing were done according to the MinION 1D 16S Barcoding Kit (SQK-RAB204) protocol. The DNA samples were amplified by PCR using specific 16S

primers (27F and 1492R) with barcodes and 5' tags that facilitate ligase-free attachment of Rapid Sequencing Adapters. The PCR products were cleaned and concentrated with Agencourt AMPure XP beads and quantified with the Qubit dsDNA HS Assay Kit using the Qubit 3.0 Fluorometer (Molecular Probes, Life Technology, Eugene, OR). The PCR products for each library were then pooled at the required ratio, Rapid sequencing adapter 
attached, loading beads added, and then loaded into the MinION flow cell. Using the MinKNOW software with real-time basecall monitoring, the sequencing was stopped at $\sim 150,000$ reads to maintain a consistent read depth between all reactor samples.

\subsubsection{Metagenomic analysis}

The MinKNOW generated Fastq files were BLASTed against the NCBI 16S bacterial database using Oxford Nanopore's cloud-based analysis platform EPI2ME with the 16S Fastq workflow. Each read was classified based on \% coverage and identity. Results were transcribed into excel datasheets where they were sorted into different taxonomy classifications (mainly phylum and genus levels) for every time point and BMP reactor. A $3 \%$ sequence dissimilarity cut-off value, commonly used to estimate the species level of phylotype diversity, was universally applied. During the study, biodiversity was estimated for the top detected phyla with a relative abundance of $\geq 0.01 \%$. Alpha biodiversity (Shannon-Wiener index, evenness, and richness) and Beta-Euclidean biodiversity were estimated and analyzed for bacteria at the phylum level. Also, the relative abundance was estimated and compared for each BMP reactor at both phylum and genus levels. The cutoff value for biodiversity analysis at the genus level was $\geq 1 \%$ relative abundance. Several interesting genera were discussed, including those with a relative abundance of $\leq 1 \%$. These genera were selected based on their function in the anaerobic digestion process, as cited in the literature, and are shown in Table S-1. Shannon-Wiener index, used to characterize the phyla diversity in a community, was calculated by finding the percentage of a phylum to the other phyla in a community, then multiplying it by the natural logarithm of this proportion. The resulting values are summed across the phyla and multiplied by -1 (Equation 4-1) (Magurran, 1988). Evenness was calculated according to Equation 4-2 
(Magurran, 1988; Mulder et al., 2004). Evenness is between 0 and 1, with 1 being complete evenness. Richness $(\mathrm{N})$ is the total number of phyla after the cut-off.

$H=-\sum_{i=1}^{N} p i \ln p i \quad$ Equation 4-1

$E=\frac{H}{H \max }=H / \ln N \quad$ Equation 4-2

Where $\quad H$ : Shannon-Wiener index

$N$ : total number of phyla

$p i$ : proportion of phylum $i$ to all other phyla

$E$ : phyla evenness

Beta-Euclidean diversity was calculated according to Equation 4-3 (Edgar, 2018).

Beta - Euclidean $=\operatorname{sqrt}\left(\sum_{1}^{N}(n k i-n k j)^{\wedge} 2\right)$

Equation 4-3

Where $\quad N$ : total number of phyla

$n k i$ : count of OTU $k$ in sample $i$

$n k j$ : count of OTU $k$ in sample $j$

\subsubsection{Statistical analyses}

A one-way analysis of variation (ANOVA) at $95 \%$ confidence level was used to study and evaluate significant differences among the sludge reactors over time; the difference was considered significant if $p$-values were $<0.05$. If there were significant differences between two reactors, the Post Hoc Tukey test was used to further separate mean values, using the same significance level (Currell \& Dowman, 2005). Three replicates were done for each analysis, and the results are reported with the average \pm standard deviations. To compare values within the same group, the coefficient of variance (CV) was applied to compare individual data set, if the $\mathrm{CV}$ is $\leq 0.05$ then the data is not significantly different (i.e., the confidence is $\geq 95 \%$ ). 


\subsection{Results and discussion}

\subsubsection{Chemical performance of the anaerobic digestion reactors}

The progression of anaerobic digestion can be examined by monitoring the change in the chemical parameters of the sludge, including total and soluble COD, TS and VS, $\mathrm{pH}$ and biogas generation. The concentrations of tCOD and SCOD (Figures S-2 and S-3) steadily decreased over time with no significant variations between the control and the other reactors (ANOVA analysis, p-values > 0.05) (Table 4-4). Also, as shown in Table 4-4, there were no major differences in the final removal efficiencies of tCOD between the control and other reactors, while sCOD removals were lower in the CuONPs reactors compared to the control and the AgNPs reactors.

The change in TS and VS was also investigated (Figures S-4 and S-5), and the results demonstrated that both TS and VS decreased over time, but at a lower percentage relative to COD. As shown in Table 4-4, the removal of each TS and, particularly, VS was lower in the reactors that had higher concentrations of AgNPs and in the reactors that had different concentrations of CuONPs, which is similar to what was observed for the sCOD. However, each TS and VS for all reactors followed similar trends over time with no significant differences when the reactors compared to the control, which suggests that the presence of AgNPs and CuONPs at different concentrations did not significantly affect their removal. ANOVA analysis showed p-values between control and other reactors were higher than 0.05 for both TS and VS over time. Similar findings were acquired in the

literature (Hou et al., 2012), such that AgNPs did not significantly impact the removal of COD during sequencing batch reactor processes of wastewater. 
Biogas generation was monitored throughout the experiment, as shown in Figure 4-1. All reactors, except $\mathrm{F}$, showed similar performance regarding the generation of biogas, with no significant variations among the reactors over time. This agrees with the results from previous studies (Yang et al., 2012; Sakarya et al., 2015) that found the presence of AgNPs (at concentrations of $13.2 \mathrm{~g}$ silver/kg biomass COD and 1, $10 \mathrm{mg} / \mathrm{g}$ TS, respectively) had no significant impact on methane production. Comparing the biogas generation for the control with the other reactors over time using T-test analysis showed that $\mathrm{p}$-values were $0.22,0.19,0.06,0.80,0.89$ and 0.006 for reactors A, B, C, D, E, and F, respectively. Biogas yield was also estimated for each reactor and the analyses showed a decrease in the reactors with higher concentrations of AgNPs and CuONPs (reactors C and F). The inhibitory impact of CuONPs on biogas generation seems to be higher than that for AgNPs, which was confirmed by several studies. For example, Luna-delRisco et al. (2011) demonstrated that the presence of $\mathrm{CuONPs}$ at $10.7 \mathrm{mg} \mathrm{Cu} / \mathrm{L}$ decreased methane production by $50 \%$ during anaerobic digestion of cattle manure for $14 \mathrm{~d}$ at $35^{\circ} \mathrm{C}$. Another study conducted by Otero-González et al. (2014) found that long term exposure (126-150 d) to CuONPs at 1.4 $\mathrm{mg} \mathrm{Cu} / \mathrm{L}$ during anaerobic digestion of synthetic wastewater at $30 \pm 2{ }^{\circ} \mathrm{C}$ also reduced methane production by more than $50 \%$ due to severe toxicity that reduced more than $85 \%$ of the acetoclastic methanogenic activity. In addition, similar findings were observed by several other researchers, including Gonzalez-Estrella et al. (2013), Ünşar et al. (2016), as was mentioned in the introduction. In general, as shown in Table 4-4, AgNPs and CuONPs did not affect tCOD removals, but impacted and decreased the biogas generation, which was supported by the decreased removal rate of the SCOD, TS, and VS in the reactors that had AgNPs and CuONPs. However, these impacts were not statistically significant, except 
for decreasing the biogas formation caused by a higher CuONPs concentration $(30 \mathrm{mg}$ NPs/g TS of sludge).
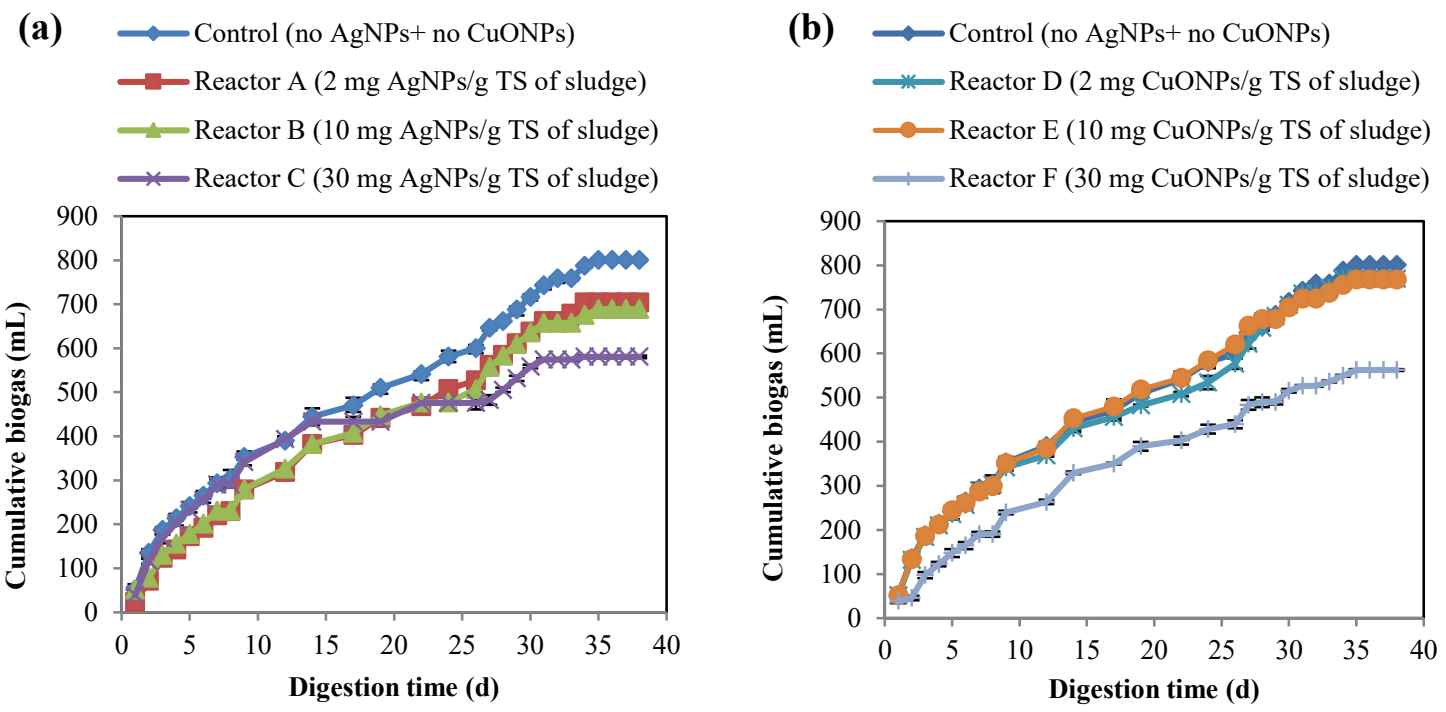

Figure 4-1 Cumulative biogas production over time for BMP reactors that had AgNPs (a) and $\mathrm{CuONPs}(\mathrm{b})$ with the control. The result showed that reactor $\mathrm{F}$ is significantly different from the control (p-value $=0.006)$.

Table 4-4 Biogas yield, tCOD, sCOD, TS, and VS reduction in the BMP reactors with and without AgNPs and CuONPs.

\begin{tabular}{|c|c|c|c|c|c|c|}
\hline Reactor & $\begin{array}{c}\text { Final Biogas } \\
\text { volume } \\
\text { generated } \\
(\mathrm{mL})\end{array}$ & $\begin{array}{l}\text { Biogas yield } \\
\text { (L biogas/kg } \\
\text { tCOD } \\
\text { removed) }\end{array}$ & $\begin{array}{c}\text { tCOD } \\
\text { reduction } \\
\%\end{array}$ & $\begin{array}{c}\text { sCOD } \\
\text { reduction } \\
\%\end{array}$ & $\begin{array}{c}\text { TS reduction } \\
\%\end{array}$ & $\begin{array}{c}\text { VS reduction } \\
\%\end{array}$ \\
\hline Control & $800.5 \pm 3$ & $278.2 \pm 13.4$ & $56.4 \pm 4.2$ & $71.1 \pm 5.2$ & $32.5 \pm 6.7$ & $50.0 \pm 3.8$ \\
\hline $\begin{array}{l}\text { Reactor A ( } 2 \mathrm{mg} \\
\text { AgNPs/g TS) }\end{array}$ & $704.0 \pm 4$ & $281.2 \pm 26.4$ & $50.8 \pm 2.7$ & $70.3 \pm 7.1$ & $31.9 \pm 2.5$ & $50.1 \pm 1.3$ \\
\hline $\begin{array}{l}\text { Reactor B (10 mg } \\
\text { AgNPs/g TS) }\end{array}$ & $689.0 \pm 5$ & $320.0 \pm 17.3$ & $43.9 \pm 2.2$ & $70.0 \pm 5.5$ & $29.6 \pm 1.7$ & $46.2 \pm 2.1$ \\
\hline $\begin{array}{l}\text { Reactor C ( } 30 \mathrm{mg} \\
\text { AgNPs/g TS) }\end{array}$ & $580.5 \pm 3$ & $229.2 \pm 15.6$ & $51.3 \pm 1.7$ & $70.0 \pm 3.4$ & $19.4 \pm 1.4$ & $49.8 \pm 1.8$ \\
\hline $\begin{array}{l}\text { Reactor D (2 mg } \\
\text { CuONPs/g TS })\end{array}$ & $769.0 \pm 2$ & $284.1 \pm 23.2$ & $53.1 \pm 2.1$ & $59.0 \pm 6.4$ & $28.2 \pm 3.4$ & $43.6 \pm 2.4$ \\
\hline $\begin{array}{l}\text { Reactor E (10 mg } \\
\text { CuONPs/g TS) }\end{array}$ & $768.0 \pm 3$ & $298.2 \pm 18.2$ & $52.1 \pm 3.1$ & $59.5 \pm 7.1$ & $27.3 \pm 5.1$ & $41.2 \pm 4.1$ \\
\hline $\begin{array}{l}\text { Reactor F (30 mg } \\
\text { CuONPs/g TS) }\end{array}$ & $562.5 \pm 2$ & $225.1 \pm 17.2$ & $50.8 \pm 3.2$ & $61.9 \pm 6.1$ & $26.3 \pm 2$ & $41.9 \pm 3.4$ \\
\hline
\end{tabular}

Data represent the mean \pm standard deviation of replicate measurements. 


\subsubsection{Impact of AgNPs and CuONPs on cell viability}

In addition to the bacterial structure analyses that are discussed in the upcoming sections, general effects of AgNPs and CuONPs on the overall sludge heterogeneous microorganisms were investigated through Live/Dead bacterial viability assay. Viable cells, those with intact membranes, will stain and fluoresce green, while cells with compromised membranes are considered non-viable, and will stain and fluoresce red under the microscope. Results of live cells percentage in sludge reactors over time are shown in Figure 4-2, and a representative Live/Dead cells image at each time point for each reactor is shown in Figures S-6 to S-12. Results showed a similar trend for all the control and other sludge reactors over time with reactors $\mathrm{E}$ and $\mathrm{F}$ having the lower live cell percentage. However, the results did not show significant statistical differences between the control and other reactors. Overall, the results showed that AgNPs had no significant impact on the live cells while CuONPs decreased the percentage of the live cells with higher concentration supporting the decrease in the biogas production with the higher concentration of CuONPs. As seen in Figure 4-2, the percentage of the live cells was decreasing in all the reactors until after day 17 , when the percentage of the live cells started to recover and increase. This was supported by DNA concentrations over time (Figure S1). This is likely due to the compensatory effect. For example, prolonged exposure to AgNPs can alter the bacterial cell membrane lipids (Hachicho et al., 2014), and cause physiological changes in bacteria that can result in antibiotic resistance. Kaweeteerawat et al. (2017) reported how AgNPs inhibit Escherichia coli and cause oxidative stress, which eventually leads to increased bacterial resistance to antibiotics due to increased stress tolerance. 

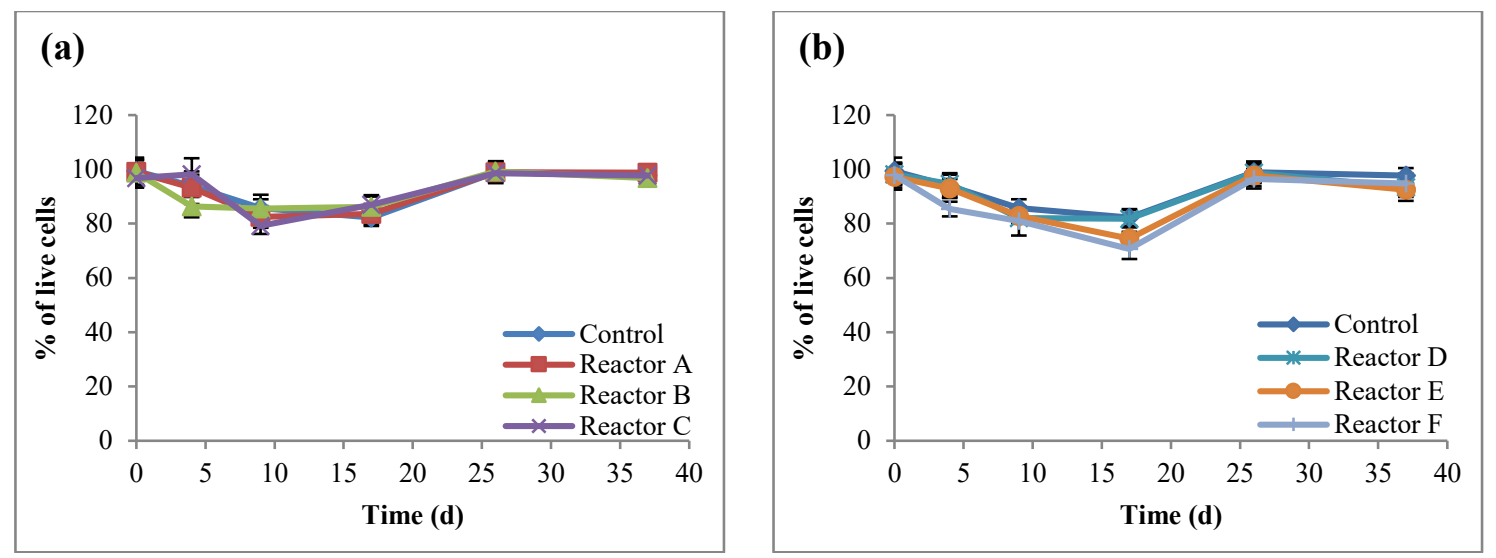

Figure 4-2 Percent of live cells (based on Live/Dead bacterial viability staining of the sludge) for the control and reactors $A, B$, and $C(a)$, and reactors $D, E$, and $F(b)$ (the control had no nanoparticles and reactors $\mathrm{A}, \mathrm{B}$, and $\mathrm{C}$ had 2, 10, and $30 \mathrm{mg} \mathrm{AgNPs} / \mathrm{g}$ TS, respectively, and reactors D, E, and $\mathrm{F}$ had 2, 10, and $30 \mathrm{mg} \mathrm{CuONPs/g} \mathrm{TS}$, respectively).

\subsubsection{Bacterial community structure in the presence of AgNPs and CuONPs}

The relative abundance and taxonomic distribution of the bacterial communities in each DNA extraction (DNA extraction concentrations are shown in Figure S-1) were mainly analyzed at the phylum and genus levels, and the results indicate that each sludge reactor had highly diverse bacterial communities. The number of species represented by unique OTUs (operational taxonomic units) tallied from all time points, were 2157, 2192, 2148, 2117, 2151, 1925 and 2045, for the control and reactors A, B, C, D, E and F, respectively. When comparing these values, it is evident that AgNPs and CuONPs at high concentrations caused a decrease in the overall species diversity in the anaerobically digested sludge. Bacterial Alpha and Beta-Euclidean biodiversity were estimated at the phylum level. Alpha diversity is a standard measure of the phyla/genera/species diversity in a community, while Beta-Euclidean diversity is a measure used to compare the composition between two communities. Alpha diversity is represented by the Shannon-Wiener index (characterizes the composition and commonness of species in a community), evenness (measures how 
close in number each species in a community is), and richness (is the number of unique OTUs or phyla/genera/species in a community). Table 4-5 shows the relationship between the concentration of nanoparticles and the number of phyla and Shannon-Wiener index in all sludge BMP reactors. Interestingly, the results showed that both the estimated ShannonWiener index and the different phyla presented were, in most cases, slightly higher in the reactors with higher concentrations of nanoparticles, suggesting that their presence may select for phyla not affected by the nanoparticles. Similar observations were reported by Huang et al. (2014) who showed an increase in the number of Shannon-Wiener index with higher doses of tetracycline. They and other researchers have stated that the antimicrobial properties of nanoparticles adversely impact the growth of the dominant bacterial species (most abundant bacteria) in the sludge allowing other species of low abundance to thrive resulting in increasing the Shannon-Wiener index and the number of different phyla presented (Huang et al., 2014; Li et al., 2011; Czárán et al., 2002). Another explanation is that these species could be nanoparticle resistant bacteria. For example, Huang et al. (2019) showed that the presence of CuONPs at $20 \mathrm{mg} / \mathrm{g}$ total suspended solids (TSS) during anaerobic digestion increased the abundance of antibiotic resistant genes (ARGs) with no significant impact on the resistance mechanisms and types. As shown in Figure 4-3, the Shannon-Wiener index for each reactor over time was also assessed, and the results showed slightly different trends among all the reactors with no significant differences (ANOVA, p-values $>0.05$ ). Figure 4-3 shows that the bacterial diversity represented by the ShannonWiener index was low for each reactor on day 0 , particularly in reactors $\mathrm{C}, \mathrm{E}$, and $\mathrm{F}$ which were lower than the control by $26.0,18.5$ and $3.5 \%$, respectively. This may be due to the toxicity of higher concentrations of AgNPs $(30 \mathrm{mg} / \mathrm{g})$ and CuONPs $(10$ and $30 \mathrm{mg} / \mathrm{g})$ in 
those three reactors. The Shannon-Wiener index increased significantly for all reactors in the first 4-9 days and then stabilized for the duration of the experiment (ANOVA test, pvalue $=0.99)$

Table 4-5 Overall diversity of the microbial population in the BMP reactors (all time points combined).

\begin{tabular}{|c|c|c|c|c|c|c|}
\hline Reactor & $\begin{array}{l}\text { Nanoparticles } \\
\text { concentration } \\
\text { (mg NPs/g TS } \\
\text { of sludge) }\end{array}$ & $\begin{array}{l}\text { Overall } \\
\text { number of } \\
\text { sequences }\end{array}$ & $\begin{array}{l}\text { Classified } \\
(\%)\end{array}$ & $\begin{array}{l}\text { Total } \\
\text { number of } \\
\text { observed } \\
\text { phyla }\end{array}$ & $\begin{array}{l}\text { Average } \\
\text { process } \\
\text { accuracy } \\
(\%)\end{array}$ & $\begin{array}{l}\text { Shannon- } \\
\text { Wiener } \\
\text { index } \\
\text { (phyla) }\end{array}$ \\
\hline Control & None & 148,735 & 83 & 24 & 83 & 1.15 \\
\hline $\begin{array}{l}\text { Reactor A } \\
\text { (AgNPs) }\end{array}$ & 2 & 147,632 & 76 & 27 & 82 & 1.12 \\
\hline $\begin{array}{l}\text { Reactor B } \\
\text { (AgNPs) }\end{array}$ & 10 & 149,972 & 87 & 27 & 83 & 1.16 \\
\hline $\begin{array}{l}\text { Reactor C } \\
\text { (AgNPs) }\end{array}$ & 30 & 143,490 & 87 & 27 & 83 & 1.14 \\
\hline $\begin{array}{l}\text { Reactor D } \\
\text { (CuONPs) }\end{array}$ & 2 & 141,444 & 89 & 27 & 83 & 1.14 \\
\hline $\begin{array}{l}\text { Reactor E } \\
\text { (CuONPs) }\end{array}$ & 10 & 136,469 & 86 & 29 & 83 & 1.17 \\
\hline $\begin{array}{l}\text { Reactor F } \\
\text { (CuONPs) }\end{array}$ & 30 & 148,759 & 85 & 23 & 83 & 1.17 \\
\hline
\end{tabular}
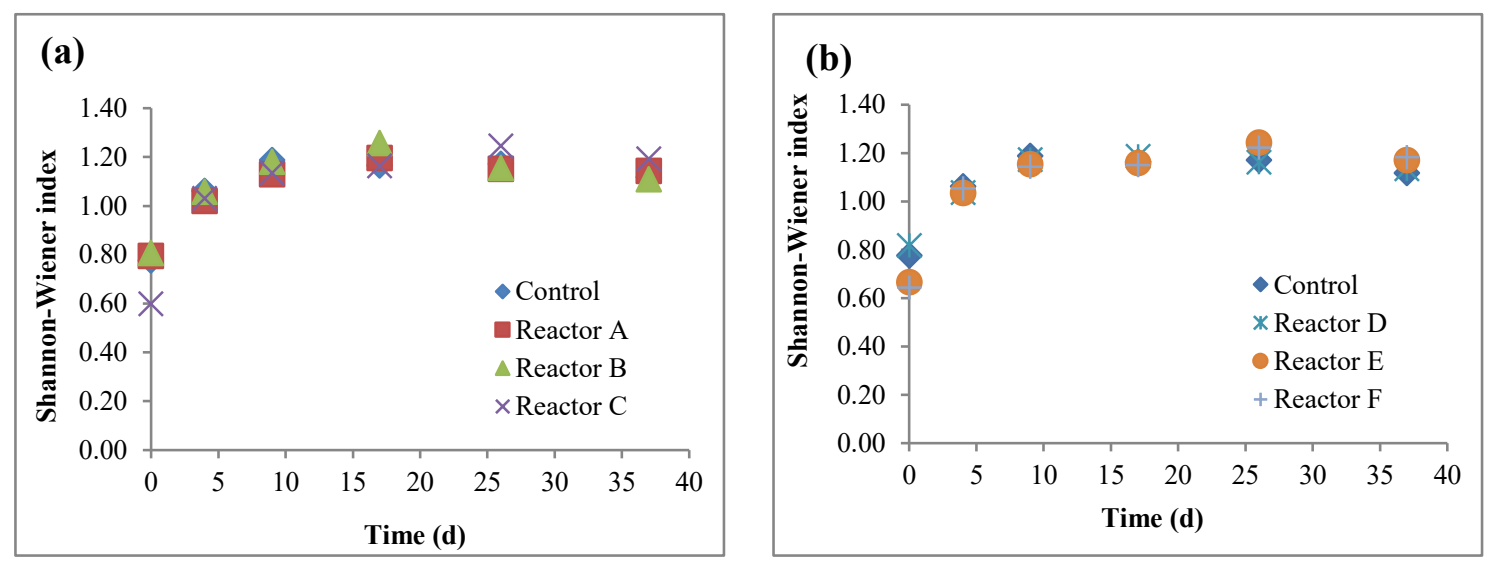

Figure 4-3 Shannon-Wiener index for the control and reactors $A, B$, and $C$ (a), and reactors

$D$, E, and $F$ (b) (the control had no nanoparticles and reactors $A$, B, and $C$ had 2, 10, and 30 mg AgNPs/g TS, respectively, and reactors D, E, and F had 2, 10, and $30 \mathrm{mg} \mathrm{CuONPs/g} \mathrm{TS,}$ respectively).

Phyla evenness and richness were also used to characterize the phyla population and diversity; the evenness is the relative abundance of phyla, and the richness represents the 
number of phyla in a community. As only phyla with $\geq 0.01 \%$ relative abundance were considered, the phyla number was 13 in each reactor, as shown in Figure 4-4(b). The evenness index was low and similar for all sludge reactors $(\mathrm{CV}=4.09 \%)$. Since the evenness index determines the diversity of a bacterial community, the results in Figure 44(a) indicate that the reactors with AgNPs and CuONPs had slightly lower diversity compared to the control.
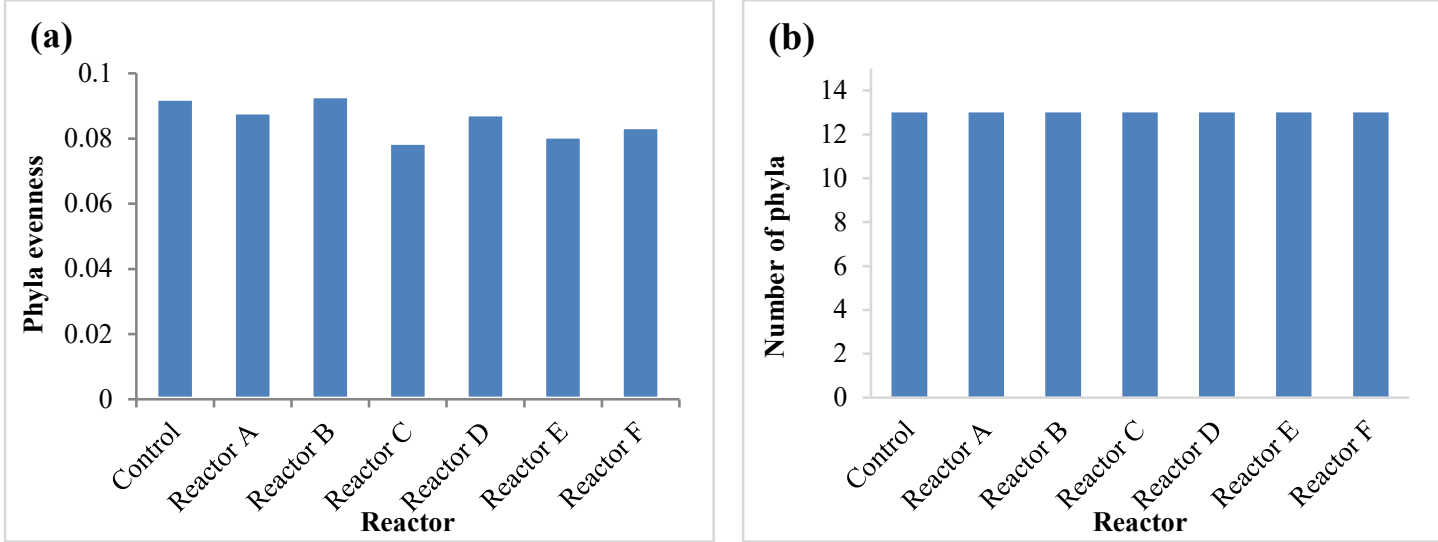

Figure 4-4 Overall phyla evenness (a) and phyla richness (represented by the number of the phyla whose relative abundance $\geq 0.01 \%$ ) (b) in the control and each reactor (reactors $A, B$, and $\mathrm{C}$ had 2, 10, and $30 \mathrm{mg}$ AgNPs/g TS, respectively, and reactors $\mathrm{D}$, E, and F had 2, 10, and $30 \mathrm{mg} \mathrm{CuONPs/g} \mathrm{TS}$, respectively).

As shown in Figure 4-5, Beta-Euclidean diversity for the phyla was also estimated by comparing the control to each other reactor, and hence examine the similarity between the control and each other reactor. The difference in the bacterial composition between the control and other reactors was higher with higher AgNPs or CuONPs concentrations, indicating that the presence of these nanoparticles can potentially affect the bacterial diversity in the anaerobically digested sludge. Thus, all studied indices showed a similar outcome: high concentrations of AgNPs and CuONPs can inhibit diversity of the detected 
phyla, including at the start of the experiment as indicated in Table 4-5 and Figures 4-3, 44 and $4-5$.

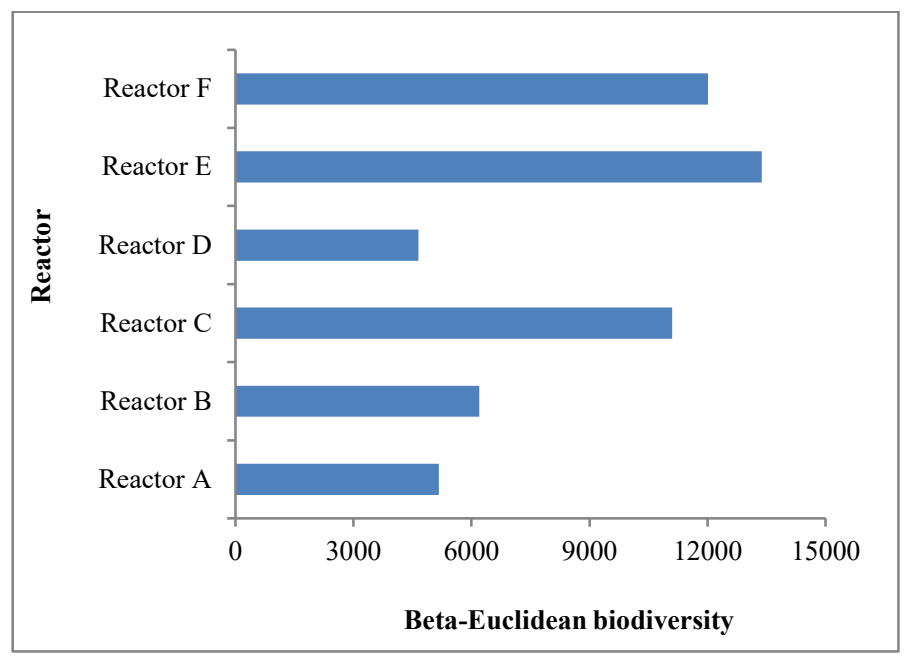

Figure 4-5 Beta-Euclidean diversity, representing the bacterial composition difference between the control and other reactors (reactors $\mathrm{A}, \mathrm{B}$, and $\mathrm{C}$ had 2, 10, and $30 \mathrm{mg} \mathrm{AgNPs} / \mathrm{g}$ TS, respectively, and reactors $\mathrm{D}, \mathrm{E}$, and $\mathrm{F}$ had 2,10 , and $30 \mathrm{mg} \mathrm{CuONPs/g} \mathrm{TS}$, respectively).

\subsubsection{Abundance and diversity of bacteria at the phylum level}

There were three dominant phyla in all of the sludge BMP reactors consisting of Proteobacteria, Firmicutes, and Bacteroidetes as shown in Figure 4-6(a). These results are consistent with other bacterial population studies measuring the abundance of bacterial phyla in anaerobically digested sludge (Kirkegaard et al., 2017; Venkiteshwaran et al., 2015; Guo et al., 2015; Yang et al., 2014). The results showed that each of the three dominant phyla had high OTUs (between 20000 to 50000 reads) with a total relative abundance of $>98 \%$, with similar trends over time in all of the sludge reactors with no significant difference between them ( $p$-value $>0.05$ ), concluding that neither AgNPs nor CuONPs seem to significantly affect their occurrence. The next most abundant group of phyla, which comprised of $\sim 2 \%$ of the total population included: Actinobacteria, Spirochaetes, Lentisphaerae, Verrucomicrobia, Planctomycetes, Chloroflexi, Fusobacteria, 
Fibrobacteres, Acidobacteria, and Synergistetes. When comparing the structure of these phyla in the control and the other sludge reactors, slight differences in their relative abundance were observed, which were due to the effect of the nanoparticles; however, these differences were not statistically significant ( $\mathrm{p}$-value $>0.05$ ) over time. Reactor B followed by the control reactor had the highest number of observed phyla OTUs among the reactors which may be due to the decrease in the diversity seen in reactors with nanoparticles as shown in Figure 4-6(b). This is in alignment with the species OTUs results discussed in Section 4.3.3, where the presence of AgNPs and CuONPs gave rise to a higher Shannon-Wiener index and increase in the overall number of phyla but resulted in lower observed unique OTUs at the species level within each phylum.
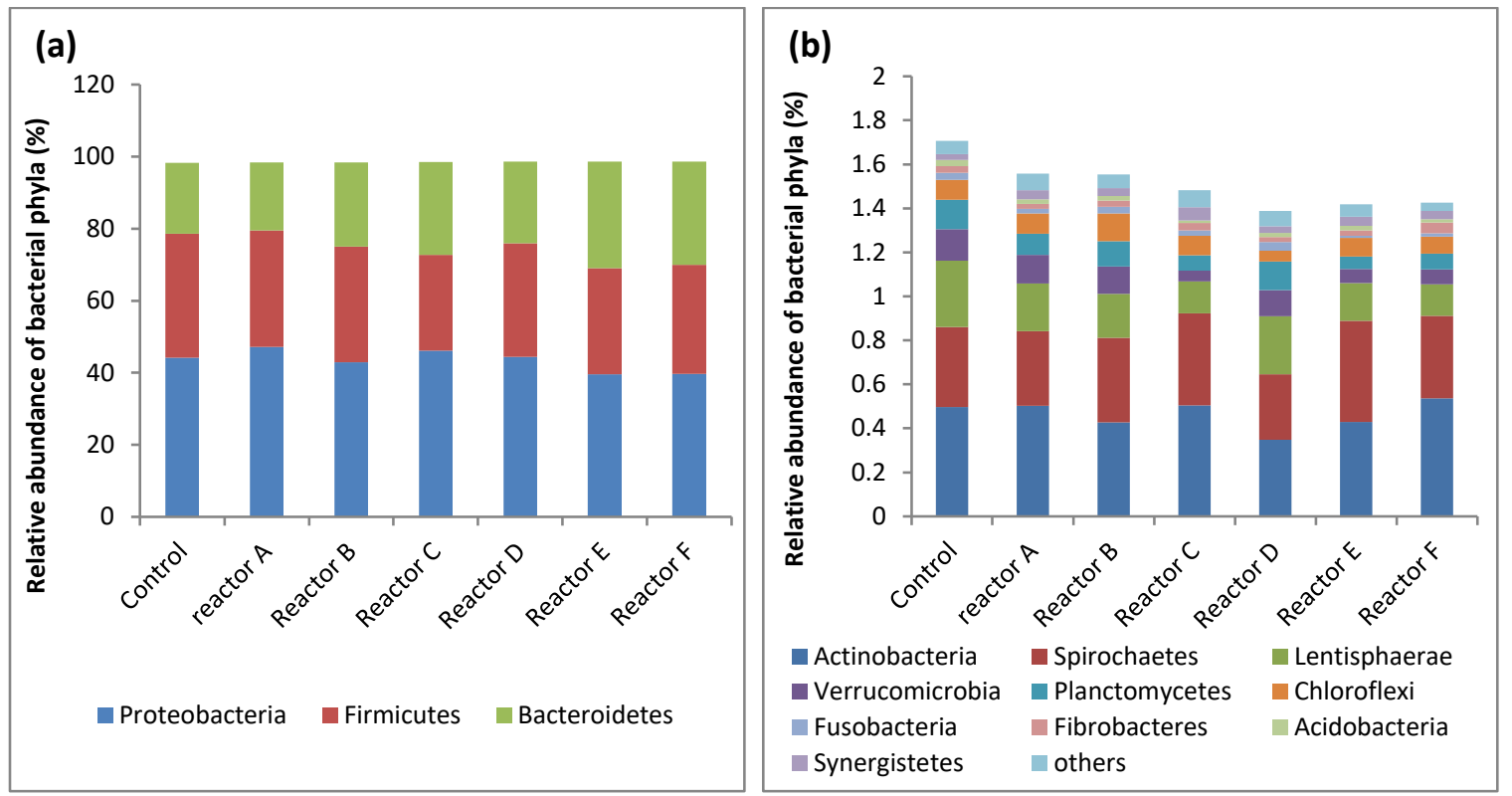

Figure 4-6 Average relative abundance at the phylum level in each sludge BMP reactor. The most abundance phyla $\geq 98 \%$ of the observed OTUs (a), and the next most abundant bacterial phyla $\leq \mathbf{2 \%}$ of the observed OTUs (b). Phyla with $\leq \mathbf{0 . 0 1 \%}$ relative abundance were not included (reactors $A, B$, and $C$ had 2, 10 and $30 \mathrm{mg} \mathrm{AgNPs/g} \mathrm{TS,} \mathrm{respectively,} \mathrm{and} \mathrm{reactors}$ D, E, and F had 2, 10 and $30 \mathrm{mg}$ CuONPs/g TS, respectively). 
The metagenomic analysis showed that the phyla impacted most, among those studied, were Bacteroidetes, Lentisphaerae, Verrucomicrobia, and Planctomycetes. The OTUs of Bacteroidetes, one of the top three most abundant phyla, increased over time in all the BMP reactors, as shown in Figure 4-7(a1). Bacteroidetes also had higher OTUs in reactors with higher concentrations of both AgNPs and CuONPs, as seen in Figure 4-7(a2). Lentisphaerae and Verrucomicrobia, members of the next most abundant group of phyla, showed a slight increase of OTUs over time in all the BMP reactors, with a lower increase in reactors C, E, and F, as seen in Figure 4-7(b1 and c1). However, their OTUs decreased with lower concentrations of both AgNPs and CuONPs, as seen in Figure 4-7(b2 and c2). Planctomycete increased in the control and reactors A, B, and D and decreased in reactors C, E, and F, as seen in Figure 4-7(d1,2) 

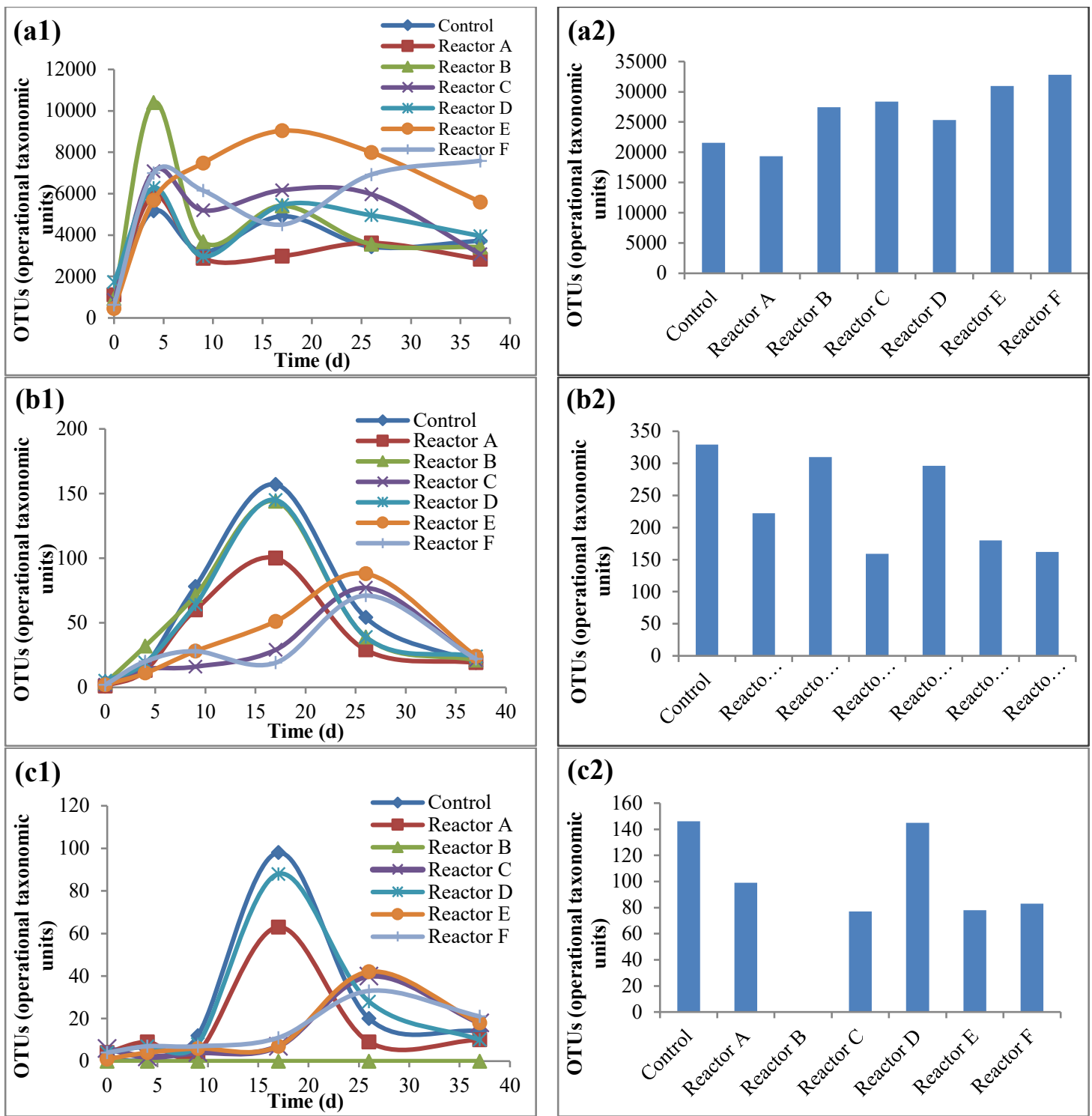

(c2)
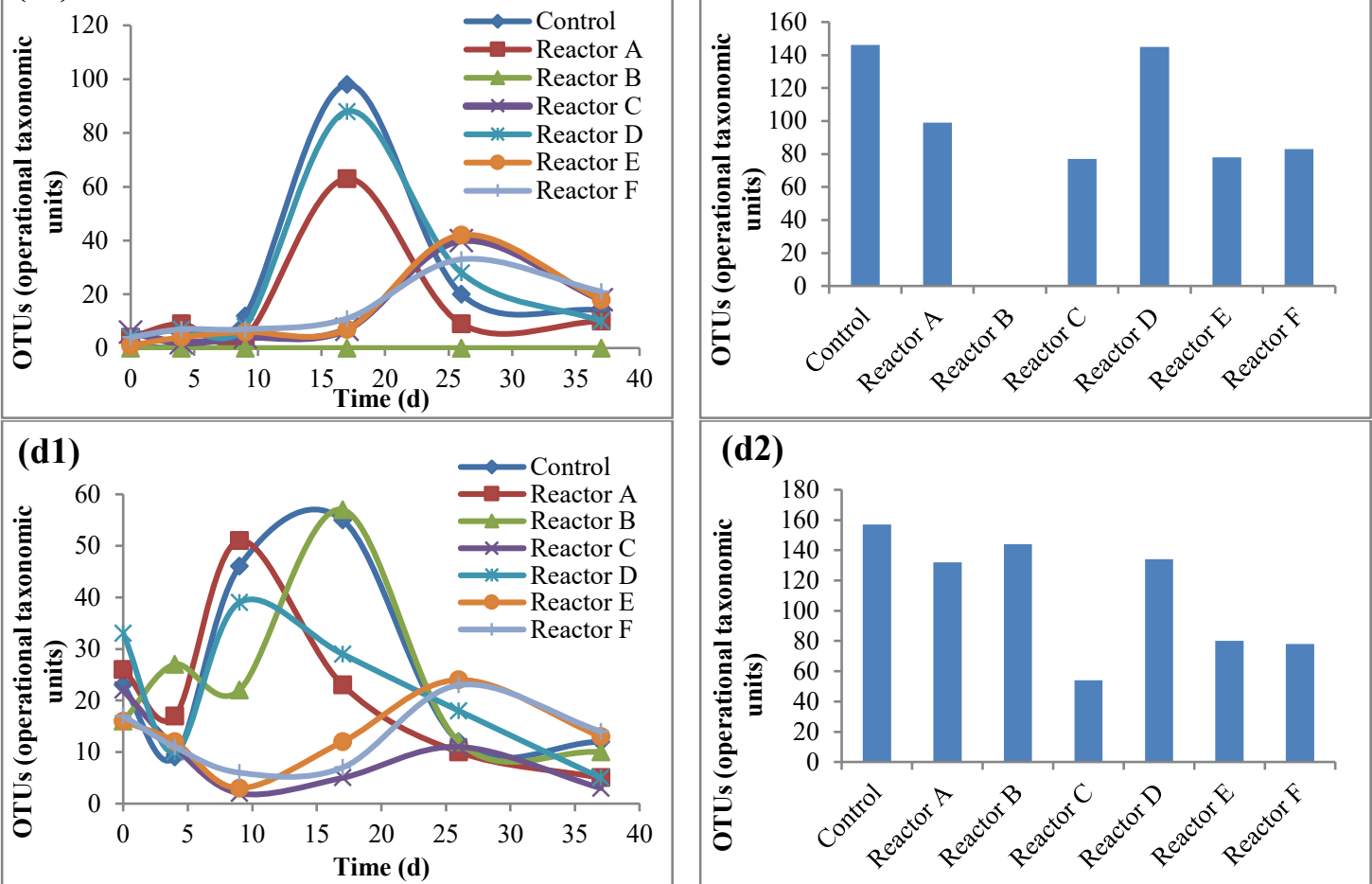

(d2)

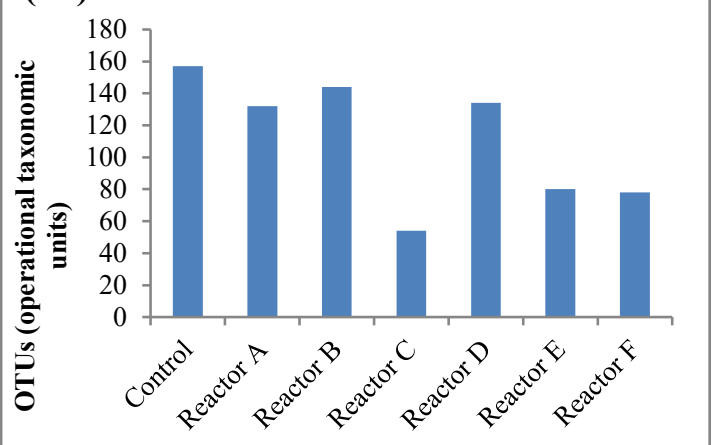

Figure 4-7 OTU prevalence over time (1), and total OTUs (2) of Bacteroidetes (a),

Lentisphaerae (b), Verrucomicrobia (c), and Planctomycete (d) for each reactor (reactors A, $\mathrm{B}$, and $\mathrm{C}$ had 2, 10, and $30 \mathrm{mg} \mathrm{AgNPs/g} \mathrm{TS}$, respectively, and reactors D, E, and F had 2, 10, and $30 \mathrm{mg} \mathrm{CuONPs/g} \mathrm{TS,} \mathrm{respectively).}$ 


\subsubsection{Abundance and diversity of bacteria at the genus level}

Several genera are discussed in this study and were chosen based on their role in anaerobic digestion of sludge. The metagenomic analysis showed that the total number of OTUs representing different genera detected throughout the experiment was $504,515,517,501$, 461,454 and 476 for the control and reactors A, B, C, D, E, and F, respectively. Thus, it is clear that the genus number was lower in reactor $\mathrm{C}$, the reactor which had the highest concentration of AgNPs, as well as and all the reactors with CuONPs (reactors D, E, and F) when compared to the control, with no statistically significant differences ( $p$-value $>$ 0.05). The top 21 genera (Figure 4-8) represent approximately $90 \%$ of the total observed OTUs; each of these genera had $\geq 1 \%$ average relative abundance in all the BMP reactors. As well, 16 genera of interest, selected from the top 75 with relative abundance between 0.1 and $1 \%$, were also analyzed in this study. Of the $37(21+16)$ studied genera, several decreased in relative abundance compared to the control, in the presence of higher concentrations of AgNPs and CuONPs; these included Novosphingobium, Syntrophus, Clostridium, Lachnoclostridium, Gracilibacter, Christensenella, Romboutsia and Syntrophobacter as shown in Figure 4-9. Conversely there were genera that seemed to thrive in the reactors with higher concentrations of AgNPs and CuONPs compared to the control which included Acidovorax, Bacteroides, Alistipes, Aeromonas, Hydrogenophaga, Sunxiuqinia, Treponema, Prolixibacter and Microbacter, as shown in Figure 4-10; other genera, that represented approximately $48-50 \%$ of the OTUs, did not show any change. In general, the shift in the bacterial community was not significant, and this supports other studies that showed no significant effect of AgNPs on the microbial community during anaerobic digestion (Doolette et al., 2013). However, it was shown in the literature that 
nanoparticle toxicity is concentration-dependent. For example, AgNPs at a concentration of $500 \mathrm{mg} / \mathrm{g}$ TSS (very high concentration) during anaerobic digestion of waste activated sludge reduced the biogas generation to $73.52 \%$ compared to the control and inhibited the population of the bacteria and archaea compared to the control (Wang et al., 2016). Also, in this study, the impact of the nanoparticles was different according to the type and the concentration of the nanoparticles used.

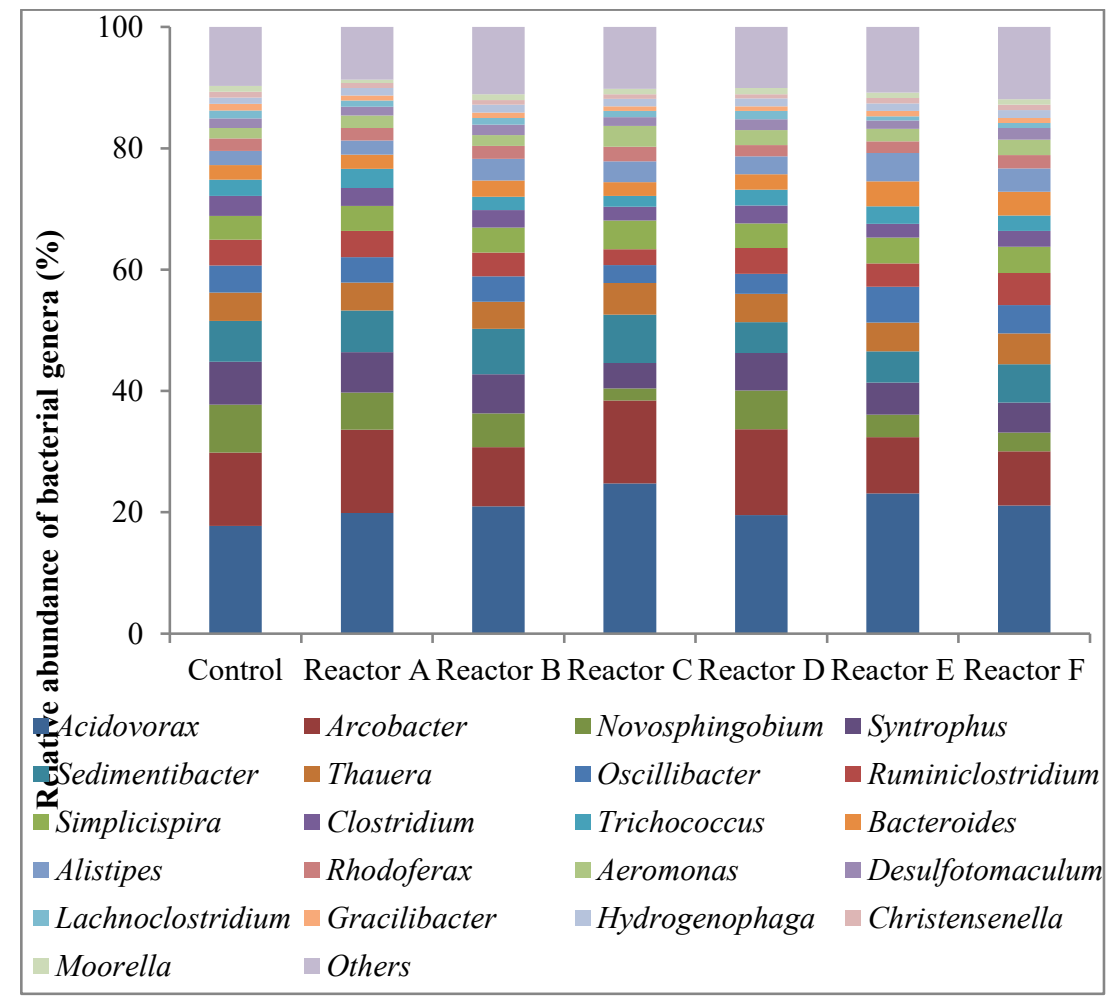

Figure 4-8 Relative abundance of the top 21 genera and those that have a relative abundance $\geq 1 \%$ (reactors $\mathrm{A}, \mathrm{B}$ and $\mathrm{C}$ had 2, 10 and $30 \mathrm{mg} \mathrm{AgNPs} / \mathrm{g}$ TS, respectively, and reactors $\mathrm{D}, \mathrm{E}$ and $\mathrm{F}$ had 2, 10 and $30 \mathrm{mg} \mathrm{CuONPs/g} \mathrm{TS}$, respectively). 


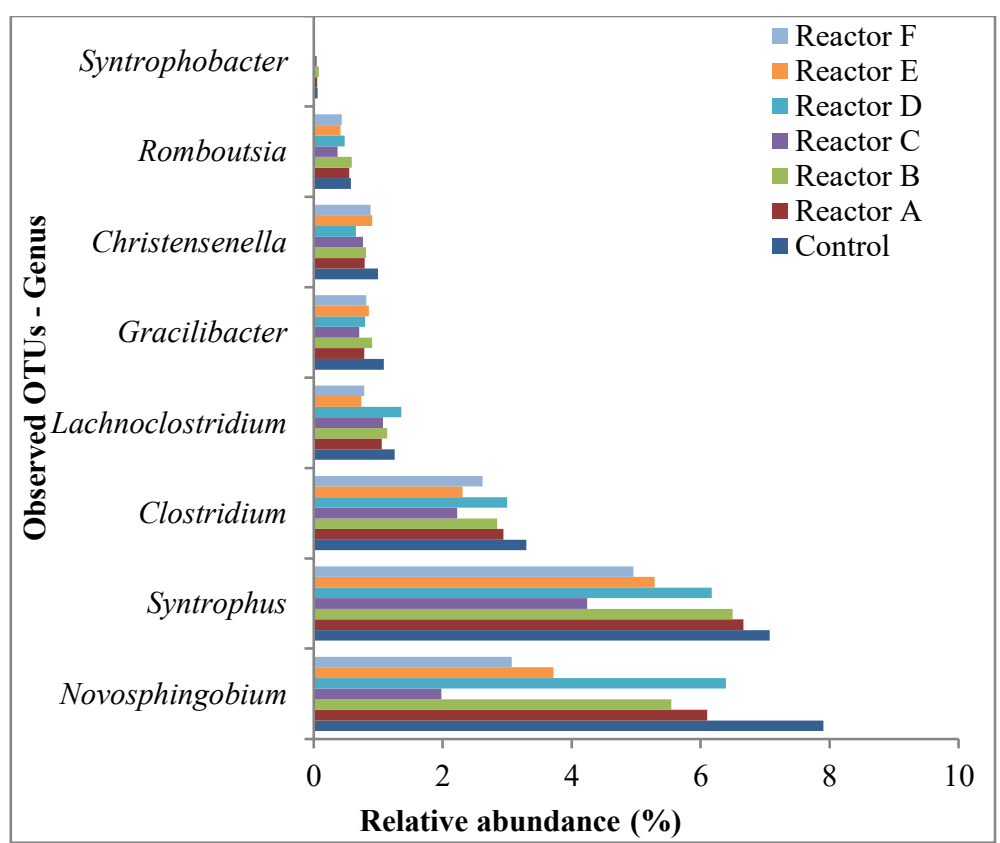

Figure 4-9 Relative abundance of genera (37 genera with relative abundance $>0.1 \%)$, that were negatively affected by the presence of the nanoparticles (reactors $\mathrm{A}, \mathrm{B}$ and $\mathrm{C}$ had 2, 10 and $30 \mathrm{mg} \mathrm{AgNPs/g} \mathrm{TS}$, respectively, and reactors D, E and F had 2, 10 and $30 \mathrm{mg} \mathrm{CuONPs/g}$ TS, respectively).

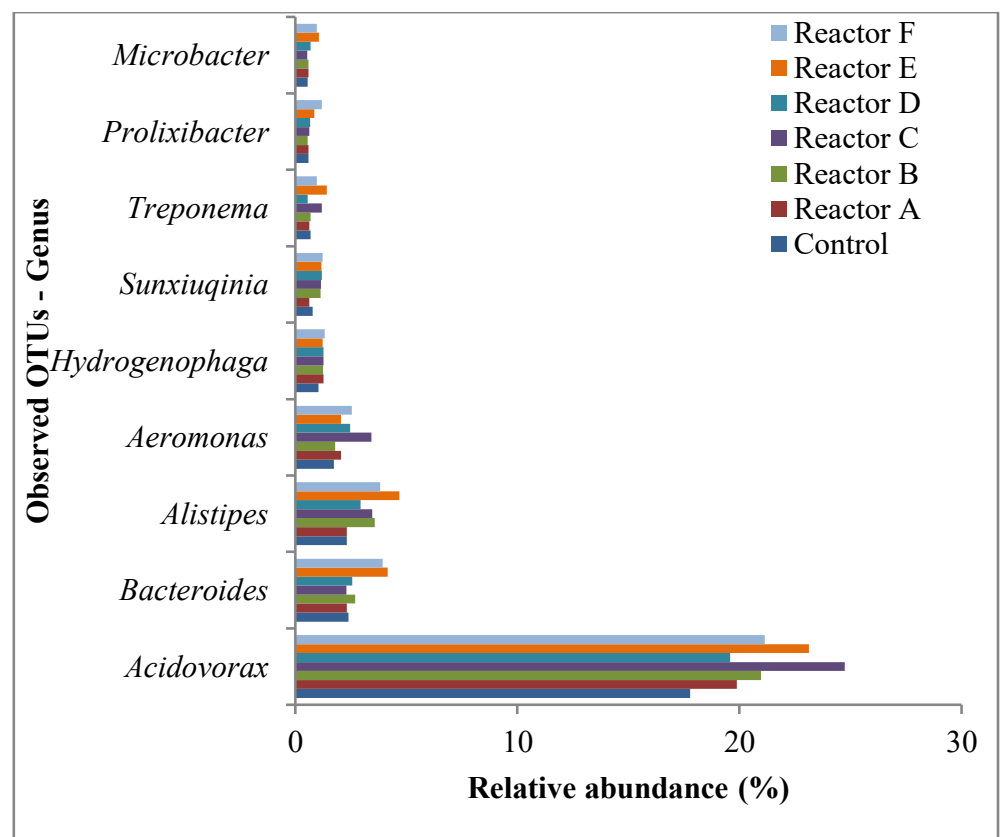

Figure 4-10 Relative abundance of genera (37 genera with relative abundance $>0.1 \%)$, that had higher observed OTUs in the presence of higher nanoparticle concentrations (reactors A, B and C had 2, 10 and $30 \mathrm{mg}$ AgNPs/g TS, respectively, and reactors D, E and F had 2, 10 and $30 \mathrm{mg} \mathrm{CuONPs/g} \mathrm{TS}$, respectively). 


\subsubsection{Key bacteria involved in the anaerobic digestion process}

At the genus level, bacterial metagenomics can provide valuable information about the functions of communities in the sludge reactor. There are four governing stages in the anaerobic digestion process: hydrolysis, fermentation, acetogenesis, and methanogenesis. The efficiency and stability of the process are entirely dependent on the diversity of bacteria that are involved in each of the four stages, and their collective synergism to achieve effective sludge reduction and methane formation. Based on a literature review (Table S1), it is clear that specific bacterial genera are involved in more than one stage of the

process. In this study, 37 bacterial genera selected from top 75 genera were studied, and their role in the sludge anaerobic digestion process was explored based on the literature, as shown in Table S-1. According to the metagenomic analysis, different bacterial genera observed are found to be involved in the processes of hydrolysis and fermentation, hydrolysis, fermentation, acetogenesis, and fermentation and acetogenesis, as well as hydrolysis, fermentation, and acetogenesis as shown in Table 4-6. 
Table 4-6 Relative abundance of bacterial genera involved in the different functional stages of the anaerobic digestion process (control had no nanoparticles and reactors $\mathrm{A}, \mathrm{B}$, and $\mathrm{C}$ had 2, 10, and $30 \mathrm{mg} \mathrm{AgNPs/g} \mathrm{TS,} \mathrm{respectively,} \mathrm{and} \mathrm{reactors} \mathrm{D,} \mathrm{E,} \mathrm{and} \mathrm{F} \mathrm{had} \mathrm{2,} \mathrm{10,} \mathrm{and} 30 \mathrm{mg}$ CuONPs/g TS, respectively).

\begin{tabular}{|c|c|c|c|c|c|c|c|c|}
\hline Function & Phylum/Genera & $\begin{array}{c}\text { Control } \\
(\%)\end{array}$ & $\begin{array}{c}\text { Reactor } \\
\text { A } \\
(\%)\end{array}$ & $\begin{array}{l}\text { Reactor } \\
\text { B } \\
(\%)\end{array}$ & $\begin{array}{c}\text { Reactor } \\
\text { C } \\
(\%)\end{array}$ & $\begin{array}{c}\text { Reactor } \\
\text { D } \\
(\%)\end{array}$ & $\begin{array}{c}\text { Reactor } \\
\text { E } \\
(\%)\end{array}$ & $\begin{array}{c}\text { Reactor } \\
\text { F } \\
(\%)\end{array}$ \\
\hline Hydrolysis & $\begin{array}{l}\text { Proteobacteria / } \\
\text { Novosphingobium. } \\
\text { Bacteroidetes / Bacteroides } \\
\text { and Sunxiuqinia. } \\
\end{array}$ & 11.1 & 9.1 & 9.4 & 5.4 & 10.2 & 9.0 & 8.2 \\
\hline Fermentation & $\begin{array}{l}\text { Firmicutes / Trichococcus, } \\
\text { Lachnoclostridium, } \\
\text { Gracilibacter, } \\
\text { Christensenella, } \\
\text { Acidaminococcus, } \\
\text { Desulfovibrio, Treponema and } \\
\text { Caldicoprobacter. } \\
\text { Bacteroidetes / Alistipes, } \\
\text { Prolixibacter and } \\
\text { Microbacter }\end{array}$ & 12.5 & 11.8 & 13.2 & 12.5 & 12.8 & 16.0 & 14.6 \\
\hline Acetogenesis & $\begin{array}{l}\text { Proteobacteria / Syntrophus. } \\
\text { Firmicutes / } \\
\text { Desulfotomaculum, Moorella, } \\
\text { Syntrophomonas, } \\
\text { Pelotomaculum } \text { and } \\
\text { Acetobacterium. }\end{array}$ & 10.7 & 9.6 & 10.6 & 8.2 & 9.8 & 8.3 & 9.6 \\
\hline $\begin{array}{l}\text { Fermentation } \\
\text { + Hydrolysis }\end{array}$ & $\begin{array}{l}\text { Proteobacteria / Acidovorax, } \\
\text { Arcobacter, Thauera, } \\
\text { Simplicispira, Rhodoferax and } \\
\text { Aeromonas. } \\
\text { Firmicutes / Oscillibacter, } \\
\text { Ruminiclostridium and } \\
\text { Faecalicatena. } \\
\text { Spirochaetes / } \\
\text { Sphaerochaeta. }\end{array}$ & 52.1 & 56.0 & 52.7 & 60.5 & 55.5 & 55.8 & 55.2 \\
\hline $\begin{array}{c}\text { Fermentation } \\
+ \\
\text { Acetogenesis }\end{array}$ & $\begin{array}{l}\text { Firmicutes / Sedimentibacter, } \\
\text { Ruminococcus, } \\
\text { Thermoanaerobacter. }\end{array}$ & 7.9 & 8.1 & 8.7 & 8.9 & 6.3 & 6.1 & 7.3 \\
\hline $\begin{array}{c}\text { Hydrolysis }+ \\
\text { Fermentation } \\
+ \\
\text { Acetogenesis }\end{array}$ & $\begin{array}{l}\text { Firmicutes / Clostridium, } \\
\text { Romboutsia, Syntrophobacter. }\end{array}$ & 3.9 & 3.5 & 3.5 & 2.6 & 3.5 & 2.7 & 3.1 \\
\hline
\end{tabular}

Table 4-6 and Figure 4-11 show that fermentation and hydrolysis were the highest occurring mechanisms during anaerobic digestion in this study, followed by acetogenesis. Fermentation bacteria, mainly Proteobacteria phylum, can assimilate amino acids, fatty acids, sugars, and alcohols, and it has been shown that this phylum is less susceptible to environmental changes than others (Ariesyady et al., 2007) which may explain the 
corresponding high OTUs observed. Firmicutes phylum included a wide range of syntrophic bacteria that play an important role in anaerobic sludge digestion, including the assimilation of different VFAs (Garcia-Peña et al., 2011). The results also showed many hydrolysis and fermentation bacteria belonging to Bacteroidetes phylum played a significant role in the anaerobic digestion process, including decomposition and conversion of organic materials into organic acids, $\mathrm{CO}_{2}$ and $\mathrm{H}_{2}$ (Traversi et al., 2012, Yang et al., 2014). 


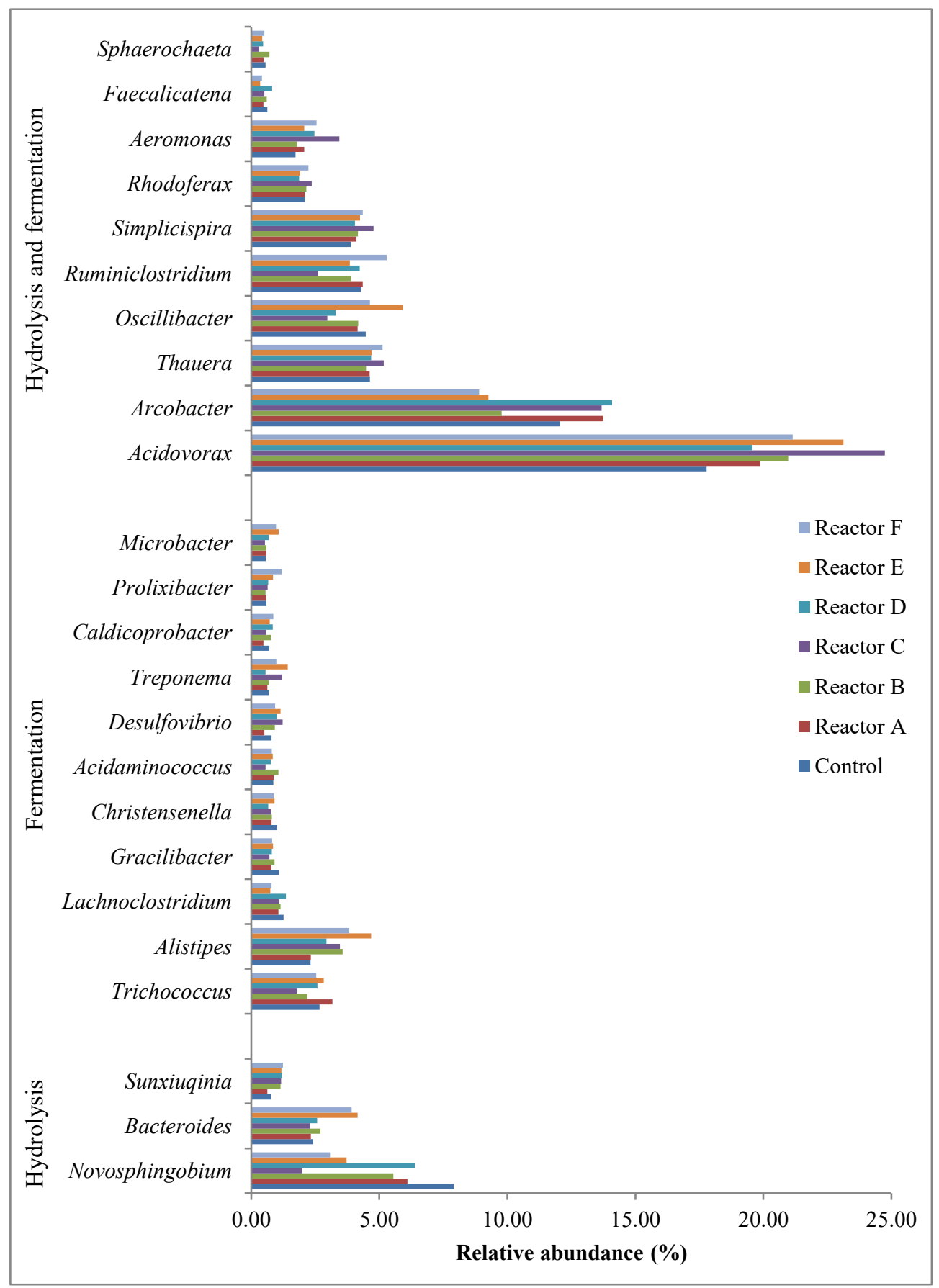

Figure 4-11 Relative abundance of bacterial genera involved in hydrolysis, fermentation, and hydrolysis and fermentation (reactors $A, B$ and $C$ had 2, 10, and $30 \mathrm{mg} \mathrm{AgNPs/g} \mathrm{TS}$, respectively, and reactors $\mathrm{D}, \mathrm{E}$, and $\mathrm{F}$ had 2,10 , and $30 \mathrm{mg} \mathrm{CuONPs} / \mathrm{g} \mathrm{TS}$, respectively). 


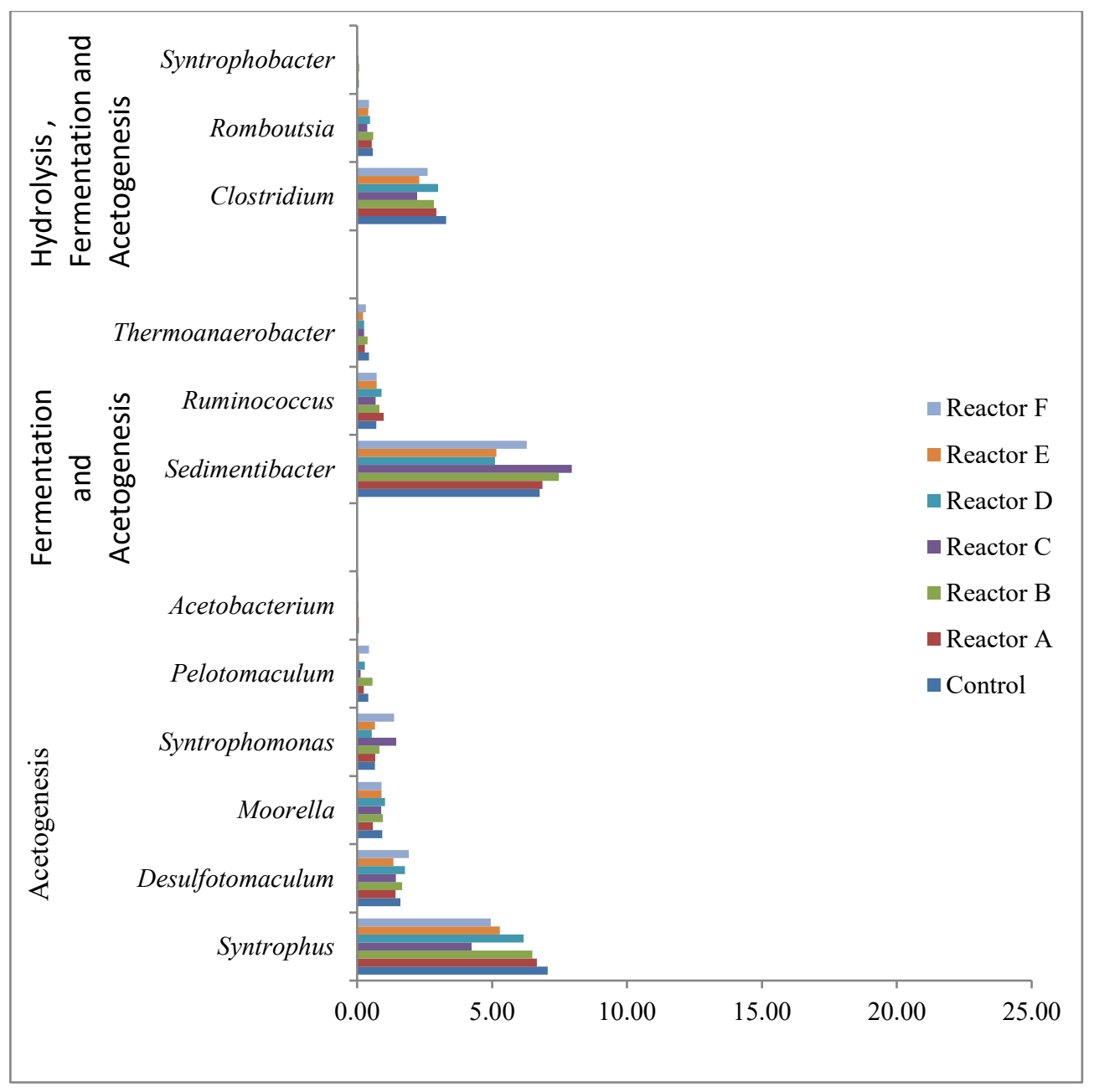

Figure 4-12 Relative abundance of bacterial genera involved in acetogenesis, fermentation and acetogenesis, and hydrolysis, fermentation, and acetogenesis (reactors $\mathrm{A}, \mathrm{B}$, and $\mathrm{C}$ had 2, 10, and $30 \mathrm{mg} \mathrm{AgNPs/g} \mathrm{TS,} \mathrm{respectively,} \mathrm{and} \mathrm{reactors} \mathrm{D,} \mathrm{E,} \mathrm{and} \mathrm{F} \mathrm{had} \mathrm{2,} \mathrm{10,} \mathrm{and} 30 \mathrm{mg}$ CuONPs/g TS, respectively).

In general, the results showed that the bacterial genera responsible for hydrolysis and fermentation had a higher relative abundance in sludge reactors containing AgNPs or CuONPs compared to the control, as indicated in Table 4-6. For example, the genus Acidovorax was the highest occurring taxa in all reactors with lower OTUs in the control reactor. In addition, the genus Arcobacter was found to be the second most prevalent genus in all sludge reactors, with higher OTUs observed in some reactors that had AgNPs or CuONPs compared to the control. These two genera belong to the phylum Proteobacteria 
and are facultative anaerobic bacteria that utilize nitrate or acetate as electron acceptors to grow under anaerobic conditions and can assimilate organic matter, fatty acids, sugars and proteins/amino acids (Schulze et al., 1999; Vandamme et al., 1992; Roalkvam et al., 2015). As shown in Figure 4-11, there were several genera involved in each function of the anaerobic digestion process that appear not to be affected by the presence of AgNPs and CuONPs. The acetogenesis stage of the process was attributed to the least abundant genera assessed $(0.1-1 \%$ relative abundance) which appeared to be lower in abundance in the presence of higher levels of AgNPs and CuONPs as most acetogenesis associated genera showed lower levels of observed OTUs, as shown in Figure 4-12. For example, the relative abundance of Syntrophus, Sedimentibacter, and Clostridium genera were lower in the presence of higher concentrations of AgNPs or/and CuONPs. These genera represent a broad class of syntrophic bacteria that have an essential role in the anaerobic decomposition of organic matter to methane and carbon dioxide, along with fermentative and methanogenic organisms (McInerney et al., 2007).

\subsubsection{Metagenomic analysis of select genera}

Prevalence of genera OTUs over time followed similar trends overall with no significant differences among all sludge reactors. However, several genera showed different trends which slightly varied from that of the control, including Acidovorax, Sedimentibacter, Syntrophus, Clostridium, Syntrophomonas, Sunxiuqinia, Prolixibacter, Acidaminococcus, Microbacter, and Geobacter, as shown in Figure 4-13. Sedimentibacter baseline OTUs were similar at the onset of the experiment but steadily increased over time, with the greatest observed OTUs in the AgNPs reactors. The increase in Sedimentibacter OTU percentages at day 37 was $326,327.2,374.8,381.5,251.3,257.6$ and $283.2 \%$ for the control 
and reactors A, B, C, D, E, and F, respectively. Syntrophus and Clostridium showed that higher concentrations of AgNPs and CuONPs might be responsible for lower OTUs for both. For example, Syntrophus showed a 27\% higher observed OTUs in reactor C compared to $62 \%$ higher in the control reactor over the 37 days and Clostridium showed a 5\% lower observed OTUs in control, while reactors $\mathrm{C}$ and $\mathrm{F}$ showed reduced observed OTUs of 62 and 40.5\%, respectively. The genera Syntrophomonas and Sunxiuqinia showed higher observed OTUs over time in all reactors with higher OTU percentage prevalence in the reactors with AgNPs and CuONPs. Syntrophomonas observed OTUs increased by 17,000 and $16,600 \%$ in reactors $\mathrm{C}$ and $\mathrm{F}$, respectively, while the control increased by $6,800 \%$. The increase in Sunxiuqinia OTUs was 1,825 and $2,350 \%$ for reactors $\mathrm{C}$ and F, respectively, compared to $925 \%$ for the control. Prolixibacter and Microbacter genera showed the highest variation in OTUs in the CuONPs reactors. The increase of Prolixibacter OTUs in reactor F was 5,550\% compared to $400 \%$ in the control and was $228.6 \%$ compared to $25 \%$ in the control for Microbacter. Acidaminococcus and Geobacter genera also showed similar trends over time in all reactors where higher concentrations of AgNPs and CuONPs seemed to decrease the prevalence of OTUs as shown in Figure 4-13. Acidaminococcus OTUs increased by 12.5 and $27 \%$ in reactors $\mathrm{C}$ and $\mathrm{F}$, respectively, compared to the control increase of $30 \%$. The OTUs percentage increase of Geobacter was 88 and $1,060 \%$ in reactors $\mathrm{C}$ and $\mathrm{F}$, respectively compared to $76.5 \%$ in control. Although there were, in some cases, marked differences in baseline OTUs over time, all sludge reactors showed similar trends after a certain amount of time. This indicates that AgNPs and $\mathrm{CuONPs}$ at the three tested concentrations did not have a significant impact on the population of these genera over time. 

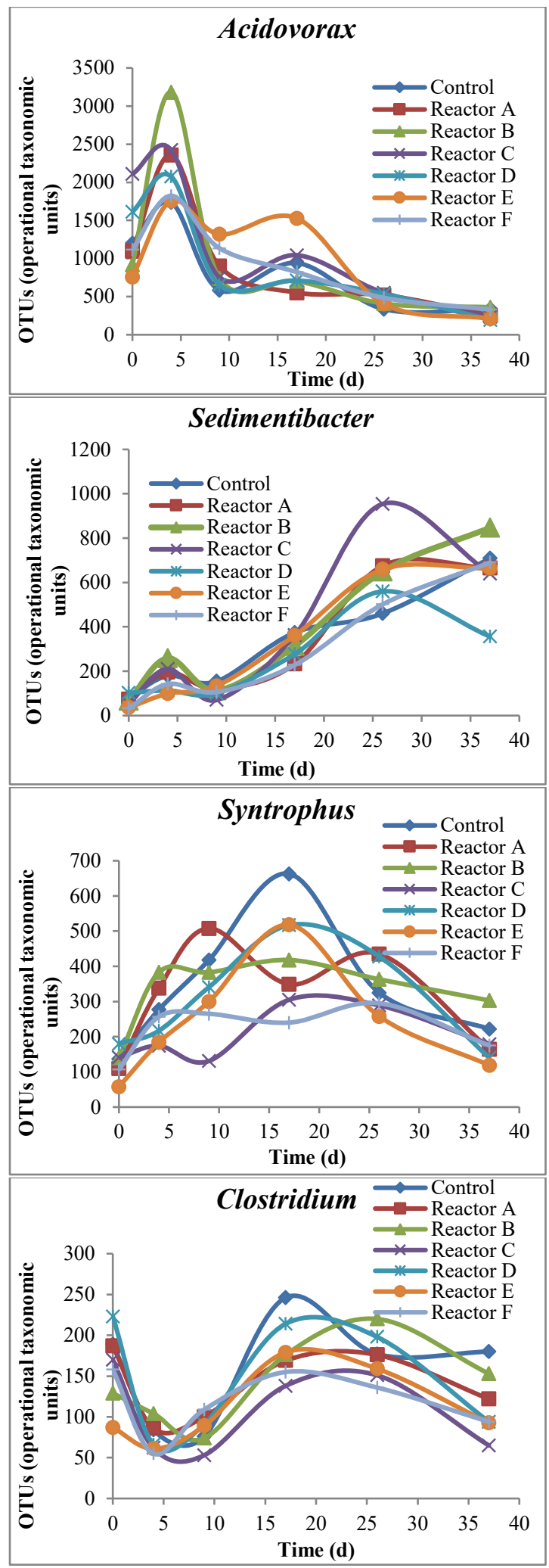
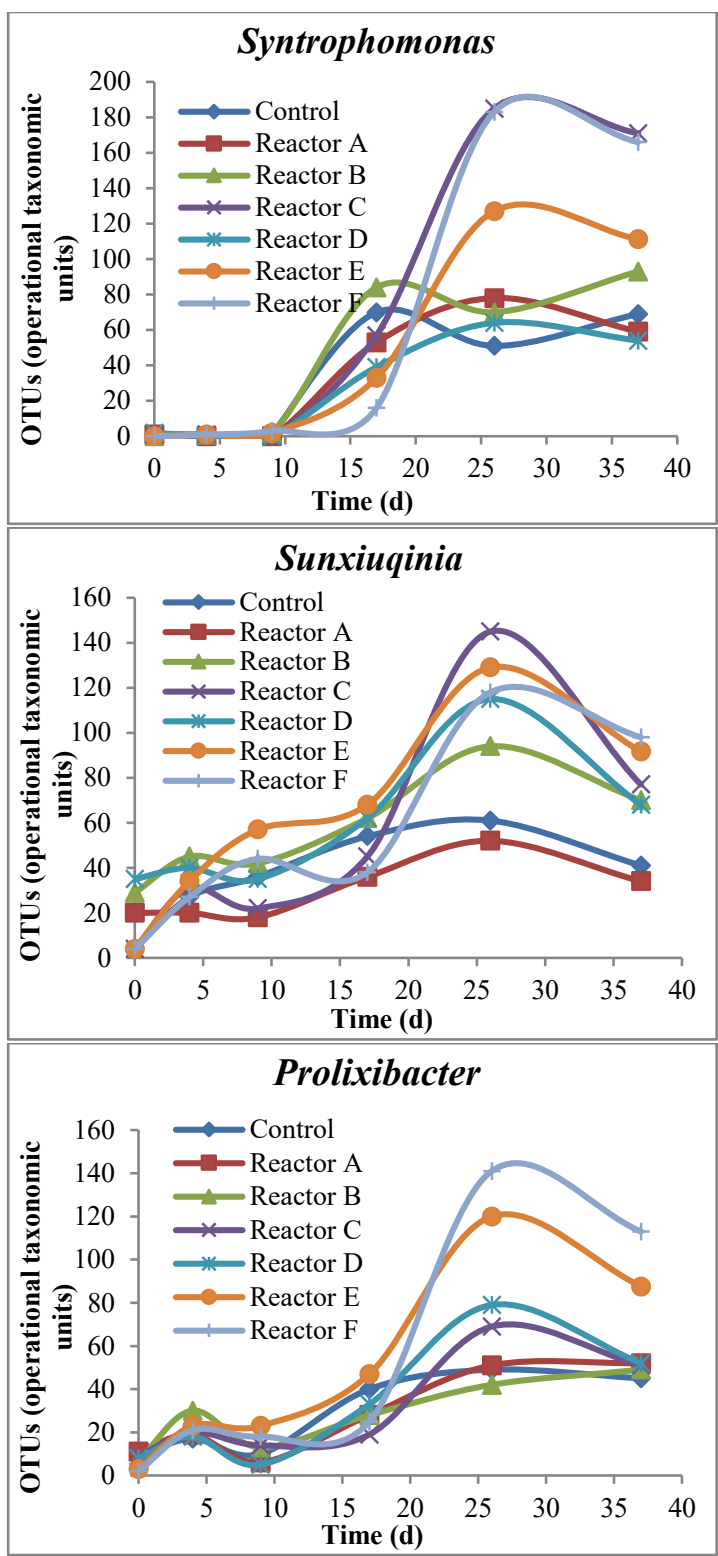

Acidaminococcus

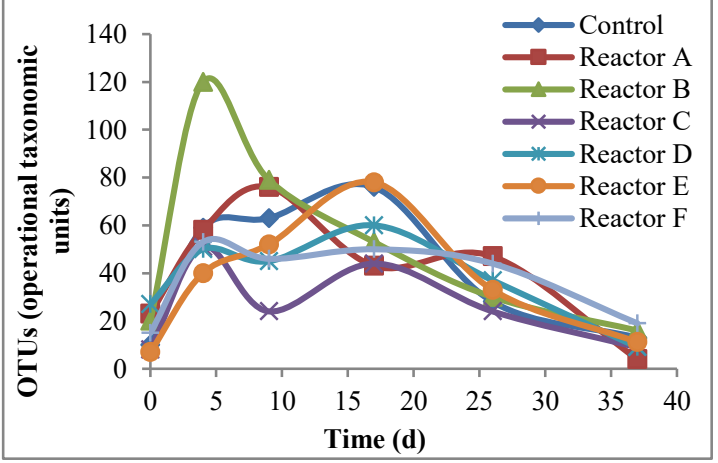

Figure 4-13 Trends of select genera over time for each BMP sludge reactor (reactors A, B, and $\mathrm{C}$ had 2, 10, and $30 \mathrm{mg} \mathrm{AgNPs/g} \mathrm{TS}$, respectively, and reactors D, E, and F had 2, 10, and $30 \mathrm{mg} \mathrm{CuONPs} / \mathrm{g} \mathrm{TS}$, respectively). 

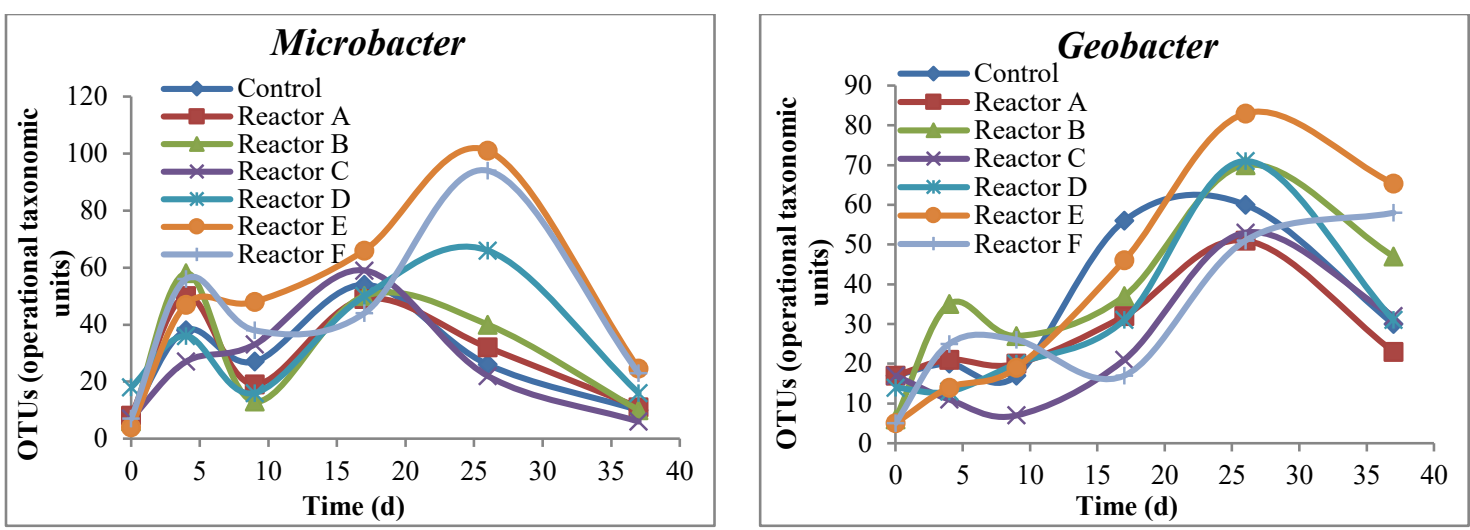

Figure 4-13 (Continued).

\subsubsection{Discussion of the relationship between bacterial function and chemical performance of the reactors}

The metagenomic sequencing results (Table 4-6) showed that the relative abundance of the bacteria responsible for hydrolysis and fermentation was the highest and increased with higher $\mathrm{AgNP}$ and $\mathrm{CuONP}$ concentrations, whereas the relative abundance of acetogenic bacteria was least and decreased with higher nanoparticle concentrations. This is likely due to the different characteristics of bacteria that were involved in these stages, for example, hydrolytic and fermentative bacteria are phylogenetically diverse, grow rapidly, and have lower sensitivity to environmental changes, while acetogenic bacteria live in syntrophy with methanogens and are more susceptible to environmental changes (Venkiteshwaran et al., 2016). Comparing the biogas generation from the reactors, and although not statistically significant, the results indicated slight differences between the control and the other sludge reactors that had AgNPs and CuONPs, except the reactor that had the highest concentration of $\mathrm{CuONPs}$ (reactor F) (Figure 4-1). This was supported by the metagenomic analyses that showed no significant impact due to the presence of AgNPs and CuONPs. These findings agree with several studies that show the low inhibitory impact of AgNPs, while there was some inhibition due to CuONPs on methanogens (Gonzalez-Estrella et al., 2013, Ünşar et 
al., 2016). Sulfidation of AgNPs and CuONPs under anaerobic conditions may also be an explanation for low inhibition of methanogenesis. The interaction of AgNPs and CuONPs with sulfur, and the subsequent production and precipitation of silver and copper sulfide, results in a significant reduction in their toxicity due to the lower solubility of silver and copper sulfide, which have limited short-term environmental impacts (Kim et al., 2010; Levard et al., 2012; Kaegi et al., 2013; Lombi et al., 2013; Gonzalez-Estrella et al., 2015). Another factor that could interfere with the antimicrobial activity of AgNPs and CuONPs is the exposure of the microbial community to high COD concentrations during the digestion process which can diminish the antimicrobial activity and favour the growth and further acclimatization of the bacteria that were not inhibited, as well as limit the growth of some bacterial species adapted to lower COD concentrations (Jang et al., 2014). In addition, several studies have demonstrated that AgNPs or CuONPs toxicity is due to the release of their ions by oxidation (Bondarenko et al., 2012; Xiu et al., 2012; Yang, 2012a) which suggest that oxygen must be present for nanoparticles to become toxic. For example, AgNPs toxicity can be controlled by managing the availability of oxygen (Xiu et al. 2012). However, other studies have shown that the toxicity could be due to the properties of the nanoparticles themselves, including their very high surface area to volume ratio that enable them to easily bind to the bacterial cell wall and alter the membrane structure or penetrate the inside of the cell membrane and make further damage, including interaction with sulfur and phosphorus-containing compounds, such as proteins and DNA (Klasen, 2000; Lansdown, 2004). Asharani et al. (2010) investigated the impact of AgNPs on red human blood cells and observed various types of cytotoxicity that were mainly due to the effect of 
AgNPs damaging DNA and no toxic effects were observed when the cells were exposed to silver ions under the same conditions.

\subsection{Conclusions}

In this study, the impact of three different concentrations $(2,10$, and $30 \mathrm{mg} / \mathrm{g}$ TS) of each AgNPs and CuONPs were explored on the anaerobic digestion performance of sludge as well as the microbial community by a comprehensive evaluation of bacterial community structures, including their diversity, abundance, and functions using high-throughput sequencing. Overall, the observed bacterial phyla OTUs were highly diverse in all BMP reactors, and the numbers of phyla detected were between 23 and 29 with 454 to 517 different genera observed over the incubation time. The diversity was attributed to the complex mix of constituents in the anaerobic sludge that originated from domestic wastewater. Based on the experimental results, the following conclusions were drawn:

1. There were no significant impacts of AgNPs at 2, 10, and $30 \mathrm{mg} \mathrm{NPs} / \mathrm{g}$ TS of sludge and $\mathrm{CuONPs}$ at 2 and $10 \mathrm{mg} \mathrm{NPs} / \mathrm{g}$ TS of sludge on the biogas generation over time. However, CuONPs at $30 \mathrm{mg} / \mathrm{g}$ TS of sludge did significantly reduced the biogas generation over time.

2. Hydrolysis and fermentation stages had bacteria with the highest abundance, while acetogenic bacteria had the lowest abundance and AgNPs and CuONPs impacted and reduced their relative abundance. However, the impacts were not statistically significant.

3. The metagenomic analysis showed that Bacteroidetes, Firmicutes, and Proteobacteria combined, represent $98 \%$ of the total observe phyla OTUs, thus these were the most 
abundant phyla observed. These phyla showed similar prevalence trends in all sludge reactors over time with no significant difference between them ( $p$-value $>0.05)$, indicating that AgNPs and CuONPs did not significantly affect their occurrence.

4. The live/dead viability assay also showed that AgNPs and CuONPs did not have a significant impact on the percentage of the live cells in the sludge samples over time.

The study showed that sludge anaerobic digestion process could be impacted in different degrees depending on the nanoparticle type and the concentration presented. However, the impact was not significant except for BMP sludge reactor that had a high concentration of CuONPs (30 mg/g TS sludge) (reactor F), indicating that, overall, CuONPs showed a higher effect on the process than the AgNPs. This study revealed bacterial community structures and their potential functions and analysed the key microorganisms involved in hydrolysis, fermentation, and acetogenesis. Therefore, the study can provide insights into the potential impact of AgNPs and CuONPs during anaerobic digestion of sludge. For future studies, it is important to investigate the impact of AgNPs and CuONPs on the archaea community structure during the anaerobic digestion of the sludge as they are very essential for the methanogenesis stage (the final stage of the anaerobic digestion). 


\section{References}

Abbott, S. L., Cheung, W. K., \& Janda, J. M. (2003). The genus Aeromonas: biochemical characteristics, atypical reactions, and phenotypic identification schemes. Journal of Clinical Microbiology, 41(6), 2348-2357.

APHA, A. P., American water works Association, A., \& Water environment federation, W. (2017). Standard methods for the examination of water and wastewater (23 ed.). (E. Rice, R. Baird, \& A. Eaton, Eds.) Washington, DC, USA: APHA, American Public Health Association; American water works Association, AWWA; Water environment federation, WEF.

Ariesyady, H. D., Ito, T., \& Okabe, S. (2007). Functional bacterial and archaeal community structures of major trophic groups in a full-scale anaerobic sludge digester. Water Research, 41(7), 1554-1568.

Asharani, P. V., Sethu, S., Vadukumpully, S., Zhong, S., Lim, C. T., Hande, M. P., \& Valiyaveettil, S. (2010). Investigations on the structural damage in human erythrocytes exposed to silver, gold, and platinum nanoparticles. Advanced Functional Materials, 20(8), 1233-1242.

Aullo, T., Ranchou-Peyruse, A., Ollivier, B., \& Magot, M. (2013). Desulfotomaculum spp. and related gram-positive sulfate-reducing bacteria in deep subsurface environments. Frontiers in Microbiology, 4, 362.

Babu, S., Claville, M. O., \& Ghebreyessus, K. (2015). Rapid synthesis of highly stable silver nanoparticles and its application for colourimetric sensing of cysteine. Journal of Experimental Nanoscience, 10(16), 1242-1255.

Balch, W. E., Schoberth, S., Tanner, R. S., \& Wolfe, R. S. (1977). Acetobacterium, a new genus of hydrogen-oxidizing, carbon dioxide-reducing, anaerobic bacteria. International Journal of Systematic and Evolutionary Microbiology, 27(4), 355-361.

Battez, A. V., González, R., Blanco, D., Asedegbega, E., \& Osorio, A. (2010). Friction reduction properties of a $\mathrm{CuO}$ nanolubricant used as lubricant for a NiCrBSi coating. Wear, 268(12), 325-328. 
Ben David, Y., Dassa, B., Borovok, I., Lamed, R., Koropatkin, N. M., Martens, E. C., \& White, B. A. (2015). Ruminococcal cellulosome systems from rumen to human. Environmental Microbiology, 17(9), 3407-3426.

Blaser, S. A., Martin, S., Matthew, M., \& Konrad, H. (2008). Estimation of cumulative aquatic exposure and risk due to silver: contribution of nano-functionalized plastics and textiles. Science of The Total Environment, 390(2), 396-409.

Bondarenko, O., Ivask, A., Käkinen, A., \& Kahru, A. (2012). Sub-toxic effects of CuO nanoparticles on bacteria: kinetics, role of $\mathrm{Cu}$ ions and possible mechanisms of action. Environmental Pollution, 169, 81-89.

Bouanane-Darenfed, A., Hania, W. B., Cayol, J.-L., Ollivier, B., \& Fardeau, M.-L. (2015). Reclassification of Acetomicrobium faecale as Caldicoprobacter faecalis comb. nov. International Journal of Systematic and Evolutionary Microbiology, 65(10), 3286-3288.

Breitenstein, A., Wiegel, J., Haertig, C., Weiss, N., Andreesen, J. R., \& Lechner, U. (2002). Reclassification of Clostridium hydroxybenzoicum as Sedimentibacter hydroxybenzoicus gen. nov., comb. nov., and description of Sedimentibacter saalensis sp. nov. International Journal of Systematic and Evolutionary Microbiology, 52(3), 801-807.

Caccavo, F., Lonergan, D. J., Lovley, D. R., Davis, M., Stolz, J. F., \& McInerney, M. J. (1994). Geobacter sulfurreducens sp. nov., a hydrogen-and acetate-oxidizing dissimilatory metalreducing microorganism. Applied and Environmental Microbiology, 60(1), 3752-3759.

CCME, A. (2010). Review of the Current Canadian Legislative Framework for Wastewater Biosolids. Canadian Council of Ministers of the Environment (CCMC).

Chen, S., Liu, X., \& Don, X. (2005). Syntrophobacter sulfatireducens sp. nov., a novel syntrophic, propionate-oxidizing bacterium isolated from UASB reactors. International Journal of Systematic and Evolutionary Microbiology, 55(3), 1319-1324.

Chung, B. S., Ryu, S. H., Park, M., Jeon, Y., Chung, Y. R., \& Jeon, C. O. (2007). Hydrogenophaga caeni sp. nov., isolated from activated sludge. International Journal of Systematic and Evolutionary Microbiology, 57(5), 1126-1130. 
Currell, G., \& Dowman, A. (2005). Essential mathematics and statistics for science. Chichester;Hoboken, NJ: Wiley \& Sons.

Czárán, T. L., Hoekstra, R. F., \& Pagie, L. (2002). Chemical warfare between microbes promotes biodiversity. Proceedings of the National Academy of Sciences, 99(2), 786-790.

Doolette, C. L., McLaughlin, M. J., Kirby, J. K., Batstone, D. J., Harris, H. H., Ge, H., \& Cornelis, G. (2013). Transformation of PVP coated silver nanoparticles in a simulated wastewater treatment process and the effect on microbial communities. Chemistry Central Journal, $7(1), 46$.

Edgar, R. (2018). Usearch V11, Euclidean distance metric. Retrieved from drive5 Bioinformatics software and services: https://drive5.com/usearch/manual/euclidean_distance.html

Elshahed, M. S., \& McInerney, M. J. (2001). Benzoate Fermentation by the Anaerobic BacteriumSyntrophus aciditrophicus in the Absence of Hydrogen-Using Microorganisms. Applied and Environmental Microbiology, 67(12), 5520-5525.

EPA, U. S. (2009). Targeted National Sewage Sludge Survey Sampling and Analysis Technical Report. United State : EPA.

Euzéby, J. P. (1997). List of Bacterial Names with Standing in Nomenclature: a folder available on the Internet. Journal of Systematic and Evolutionary Microbiology, 47(2), 590-592.

Finneran, K. T., Johnsen, C. V., \& Lovley, D. R. (2003). Rhodoferax ferrireducens sp. nov., a psychrotolerant, facultatively anaerobic bacterium that oxidizes acetate with the reduction of Fe (III). International Journal of Systematic and Evolutionary Microbiology, 53(3), 669673.

Fosses, A., Maté, M., Franche, N., Liu, N., Denis, Y., Borne, R., . . Perret, S. (2017). A sevengene cluster in Ruminiclostridium cellulolyticum is essential for signalization, uptake and catabolism of the degradation products of cellulose hydrolysis. Biotechnology for Biofuels, $10(1), 250$.

Garcia-Peña, E. I., Parameswaran, P., Kang, D. W., Canul-Chan, M., \& Krajmalnik-Brown, R. (2011). Anaerobic digestion and co-digestion processes of vegetable and fruit residues: process and microbial ecology. Bioresource Technology, 102(20), 9447-9455. 
Garrity, G., Brenner, D. K., Staley, J., \& Krieg, N. (2005). The Proteobacteria, Part C: The Alpha, Beta-, Delta-, and Epsilonproteobacteria. Bergey's manual of systematic bacteriology (pp. 647-657). New York: Springer.

Gerritsen, J., Hornung, B., Staneva, I., Ritarl, J., Paulin, L., Rijkers, G., . . Smidt, H. (2015). Comparative genomics and functional analysis of the genus Romboutsia provides insight into adaptation to an intestinal lifestyle. In The genus Romboutsia. Genomic and functional characterisation of novel bacteria dedicated to life in the intestinal tract (pp. 188-220). Wageningen University.

Ginige, M. P., Keller, J., \& Blackall, L. L. (2005). Investigation of an acetate-fed denitrifying microbial community by stable isotope probing, full-cycle rRNA analysis, and fluorescent in situ hybridization-microautoradiography. Applied and Environmental Microbiology, 71(12), 8683-8691.

Gonzalez-Estrella, J., Puyol, D., Sierra-Alvarez, R., \& Field, J. A. (2015). Role of biogenic sulfide in attenuating zinc oxide and copper nanoparticle toxicity to acetoclastic methanogenesis. Journal of Hazardous Materials, 283, 755-763.

Gonzalez-Estrella, J., Sierra-Alvarez, R., \& Field, J. A. (2013). Toxicity assessment of inorganic nanoparticles to acetoclastic and hydrogenotrophic methanogenic activity in anaerobic granular sludge. Journal of Hazardous Materials, 260, 278-285.

Gottschalk, F., Sun, T., \& Nowack, B. (2013). Environmental concentrations of engineered nanomaterials: review of modeling and analytical studies. Environmental Pollution, 181, 287-300.

Grabovich, M., Gavrish, E., Kuever, J., Lysenko, A. M., Podkopaeva, D., \& Dubinina, G. (2006). Proposal of Giesbergeria voronezhensis gen. nov., sp. nov. and G. kuznetsovii sp. nov. and reclassification of [Aquaspirillum] anulus,[A.] sinuosum and [A.] giesbergeri as Giesbergeria anulus comb. nov., G. sinuosa comb. nov. and G. giesbergeri comb. nov. International Journal of Systematic and Evolutionary Microbiology, 56(3), 569-576.

Guo, J., Peng, Y., Ni, B.-J., Han, X., Fan, L., \& Yuan, Z. (2015). Dissecting microbial community structure and methane-producing pathways of a full-scale anaerobic reactor digesting activated sludge from wastewater treatment by metagenomic sequencing. Microbial Cell Factories, 14(1), 33. 
Hatamoto, M., Imachi, H., Fukayo, S., Ohashi, A., \& Harada, H. (2007). Syntrophomonas palmitatica sp. nov., an anaerobic, syntrophic, long-chain fatty-acid-oxidizing bacterium isolated from methanogenic sludge. International Journal of Systematic and Evolutionary Microbiology, 57(9), 2137-2142.

Holmes, D. E., Nevin, K. P., Woodard, T. L., Peacock, A. D., \& Lovley, D. R. (2007). Prolixibacter bellariivorans gen. nov., sp. nov., a sugar-fermenting, psychrotolerant anaerobe of the phylum Bacteroidetes, isolated from a marine-sediment fuel cell. International Journal of Systematic and Evolutionary Microbiology, 57(4), 701-707.

Huang, H., Chen, Y., Yang, S., \& Zheng, X. (2019). CuO and ZnO nanoparticles drive the propagation of antibiotic resistance genes during sludge anaerobic digestion: possible role of stimulated signal transduction. Environmental Science, 6(2), 528-539.

Huang, K., Tang, J., Zhang, X.-X., Xu, K., \& Ren, H. (2014). A comprehensive insight into tetracycline resistant bacteria and antibiotic resistance genes in activated sludge using nextgeneration sequencing. International Journal of Molecular Sciences, 15(6), 10083-10100.

Iino, T., Mori, K., Tanaka, K., Suzuki, K.-i., \& Harayama, S. (2007). Oscillibacter valericigenes gen. nov., sp. nov., a valerate-producing anaerobic bacterium isolated from the alimentary canal of a Japanese corbicula clam. International Journal of Systematic and Evolutionary Microbiology, 57(8), 1840-1845.

Islam, M. A., Zengler, K., Edwards, E. A., Mahadevan, R., \& Stephanopoulos, G. (2015). Investigating Moorella thermoacetica metabolism with a genome-scale constraint-based metabolic model. Integrative Biology, 7(8), 869-882.

Jang, H. M., Kim, J. H., Ha, J. H., \& Park, J. M. (2014). Bacterial and methanogenic archaeal communities during the single-stage anaerobic digestion of high-strength food wastewater. Bioresource technology, 165 , 174-182.

Jang, S.-W., Oh, M.-S., Yang, S. I., \& Cho, E.-M. (2016). Gene expression profiles of human neuroblastoma cells exposed to $\mathrm{CuO}$ nanoparticles and $\mathrm{Cu}$ ions. BioChip Journal, 10(2), $140-149$. 
Jo, H. J., Choi, J. W., Lee, S. H., \& Hong, S. W. (2012). Acute toxicity of Ag and CuO nanoparticle suspensions against Daphnia magna: the importance of their dissolved fraction varying with preparation methods. Journal of Hazardous Materials, 227, 301-308.

Juling, S., Niedzwiecka, A., Böhmert, L., Lichtenstein, D., Selve, S., Braeuning, A., . . Lampen, A. (2017). Protein corona analysis of silver nanoparticles links to their cellular effects. Journal of Proteome Research, 16(11), 4020-4034.

Kaegi, R., Voegelin, A., Ort, C., Sinnet, B., Thalmann, B., Krismer, J., . . Mueller, E. (2013). Fate and transformation of silver nanoparticles in urban wastewater systems. Water Research, 47(12), 3866-3877.

Kaegi, R., Voegelin, A., Sinnet, B., Zuleeg, S., Hagendorfer, H., Burkhardt, M., \& Siegrist, H. (2011). Behavior of metallic silver nanoparticles in a pilot wastewater treatment plant. Environmental Science \& Technology, 45(9), 3902-3908.

Keller, A. A., McFerran., S., Lazareva, A., \& Suh, S. (2013). Global life cycle releases of engineered nanomaterials. Journal of Nanoparticle Research, 15(6), 1692.

Kibbee, R. J., \& Örmeci, B. (2017). Development of a sensitive and false-positive free PMA-qPCR viability assay to quantify VBNC Escherichia coli and evaluate disinfection performance in wastewater effluent. Journal of Microbiological Methods, 132, 139-147.

Kim, B., Park, C.-S., Murayama, M., \& Hochella Jr., M. F. (2010). Discovery and characterization of silver sulfide nanoparticles in final sewage sludge products. Environmental Science \& Technology, 44(19), 7509-7514.

Kirkegaard, R. H., McIlroy, S. J., Kristensen, J. M., Nierychlo, M., Karst, S. M., Dueholm, M. S., ... Nielsen, P. H. (2017). Identifying the abundant and active microorganisms common to full scale anaerobic digesters. Biorxiv, 104620.

Klasen, H. J. (2000). A historical review of the use of silver in the treatment of burns. Burns, 26(2), 131-138.

Lansdown, A. B. (2004). A review of the use of silver in wound care: facts and fallacies. British Journal of Nursing, 13(1), S6-S19. 
Lee, Y.-J., Romanek, C. S., Mills, G. L., Davis, R. C., Whitman, W. B., \& Wiegel, J. (2006). Gracilibacter thermotolerans gen. nov., sp. nov., an anaerobic, thermotolerant bacterium from a constructed wetland receiving acid sulfate water. International Journal of Systematic and Evolutionary Microbiology, 56(9), 2089-2093.

Levard, C., Hotze, E. M., Lowry, G. V., \& Brown Jr., G. E. (2012). Environmental transformations of silver nanoparticles: impact on stability and toxicity. Environmental Science \& Technology, 46(13), 6900-6914.

Li, A., Chu, Y., Wang, X., Ren, L., Yu, J., Liu, X., . . Li, S. (2013). A pyrosequencing-based metagenomic study of methane-producing microbial community in solid-state biogas reactor. Biotechnology for Biofuels, 6(1), 3.

Li, D., Qi, R., Yang, M., Zhang, Y., \& Yu, T. (2011). Bacterial community characteristics under long-term antibiotic selection pressures. Water Research, 45(18), 6063-6073.

Li, L., Stoiber, M., Wimmer, A., Xu, Z., Lindenblatt, C., Helmreich, B., \& Schuster, M. (2016). To what extent can full-scale wastewater treatment plant effluent influence the occurrence of silver based nanoparticles in surface waters? Environmental Science \& Technology.

Liang, Z., Das, A., \& Hu, Z. (2010). lombi. Water Research, 44(18), 5432-5438.

Lombi, E., Donner, E., Taher, S., Tavakkoli, E., Jämting, Å. K., McClure, S., . . Vasilev, K. (2013). Transformation of four silver/silver chloride nanoparticles during anaerobic treatment of wastewater and post-processing of sewage sludge. Environmental Pollution, 176, 193-197.

Luna-delRisco, M., Orupõld, K., \& Dubourguier, H.-C. (2011). machi. Journal of Hazardous Materials , 189(1-2), 603-608.

Imachi, H. S., Ohashi, A., Harada, H., Hanada, S., Kamagata, Y., \& Sekiguchi, Y. (2007). Pelotomaculum propionicicum sp. nov., an anaerobic, mesophilic, obligately syntrophic, propionate-oxidizing bacterium. International Journal of Systematic and Evolutionary Microbiology, 57(7), 1487-1492.

Magurran, A. E. (1988). Ecological diversity and its measurement. Princeton university press.

McInerney, M. J., Rohlin, L., Mouttaki, H., Kim, U., Krupp, R. S., Rios-Hernandez, L., . . . Gunsalus, R. (2007). The genome of Syntrophus aciditrophicus: life at the thermodynamic 
limit of microbial growth. Proceedings of the National Academy of Sciences, 104(18), $7600-7605$.

Metcalf, L., \& Eddy, H. P. (2003). Waste Water Engineering: Treatment and Reuse (4 ed.). New York: McGraw-Hill.

Morotomi, M., Nagai, F., \& Watanabe, Y. (2012). Description of Christensenella minuta gen. nov., sp. nov., isolated from human faeces, which forms a distinct branch in the order Clostridiales, and proposal of Christensenellaceae fam. nov. International Journal of Systematic and Evolutionary Microbiology, 62(1), 144-149.

Mulder, C., Bazeley-White, E., D., P.G., H. A., Scherer-Lorenzen, M., \& Schmid, B. (2004). Species evenness and productivity in experimental plant communities. Oikos, 107(1), 5063.

Nagai, F., Morotomi, M., Watanabe, Y., Sakon, H., \& Tanaka, R. (2010). Alistipes indistinctus sp. nov. and Odoribacter laneus sp. nov., common members of the human intestinal microbiota isolated from faeces. International journal of systematic and evolutionary microbiology, 60(2), 1296-1302.

Otero-González, L., Field, J. A., \& Sierra-Alvarez, R. (2014). Inhibition of anaerobic wastewater treatment after long-term exposure to low levels of $\mathrm{CuO}$ nanoparticles. Water Research, $58,160-168$.

Pikuta, E. V., Hoover, R. B., Bej, A. K., Marsic, D., Whitman, W. B., Krader, P. E., \& Tang, J. (2006). Trichococcus patagoniensis sp. nov., a facultative anaerobe that grows at "5 6C, isolated from penguin guano in Chilean Patagoniac. International Journal of Systematic and Evolutionary Microbiology, 56(9), 2055-2062.

Qiu, G., Wirianto, K., Sun, Y., \& Ting, Y.-P. (2016). Effect of silver nanoparticles on system performance and microbial community dynamics in a sequencing batch reactor. Journal of Cleaner Production, 130, 137-142.

Rasool, K., \& Lee, D. S. (2016). Inhibitory effects of silver nanoparticles on removal of organic pollutants and sulfate in an anaerobic biological wastewater treatment process. Journal of Nanoscience and Nanotechnology, 16(5), 4456-4463. 
Ritalahti, K. M., Justicia-Leon, S. D., Cusick, K. D., Ramos-Hernandez, N., Rubin, M., Dornbush, J., \& Löffler, F. E. (2012). Sphaerochaeta globosa gen. nov., sp. nov. and Sphaerochaeta pleomorpha sp. nov., free-living, spherical spirochaetes. International Journal of Systematic and Evolutionary Microbiology, 62(1), 210-216.

Roalkvam, I., Drønen, K., Stokke, R., Daae, F. L., Dahle, H., \& Steen, I. H. (2015). Physiological and genomic characterization of Arcobacter anaerophilus IR-1 reveals new metabolic features in Epsilonproteobacteria. Frontiers in Microbiology, 6, 987.

Sakamoto, M., Iino, T., \& Ohkuma, M. (2017). Faecalimonas umbilicata gen. nov., sp. nov., isolated from human faeces, and reclassification of Eubacterium contortum, Eubacterium fissicatena and Clostridium oroticum as Faecalicatena contorta gen. nov., comb. nov., Faecalicatena fissicatena comb. nov. . International Journal of Systematic and Evolutionary Microbiology, 67(5), 1219-1227.

Sakarya, K., Akyol, Ç., \& Demirel, B. (2015). The effect of short-term exposure of engineered nanoparticles on methane production during mesophilic anaerobic digestion of primary sludge. Water, Air, \& Soil Pollution, 226(4), 100.

Sanchez-Andrea, I., Sanz, J. L., \& Stams, A. J. (2014). Microbacter margulisiae gen. nov., sp. nov., a propionigenic bacterium isolated from sediments of an acid rock drainage pond. International Journal of Systematic and Evolutionary Microbiology, 64(12), 3936-3942.

Schulze, R., Spring, S., Amann, R., Huber, I., Ludwig, W., Schleifer, K.-H., \& Kämpfer, P. (1999). Genotypic diversity of Acidovorax strains isolated from activated sludge and description of Acidovorax defluvii sp. nov. Systematic and Applied Microbiology, 22(2), 205-214.

Shafer, M. M., Joel T., O., \& David E., A. (1998). Removal, partitioning, and fate of silver and other metals in wastewater treatment plants and effluent-receiving streams. Environmental Toxicology and Chemistry, 17(4), 630-641.

Sun, X., Sheng, Z., \& Liu, Y. (2013). Effects of silver nanoparticles on microbial community structure in activated sludge. Science of the Total Environment , 443, 828-835.

Takai, K., Abe, M., Miyazaki, M., Koide, O., Nunoura, T., Imachi, H., . . Kobayashi, T. (2013). Sunxiuqinia faeciviva sp. nov., a facultatively anaerobic organoheterotroph of the 
Bacteroidetes isolated from deep subseafloor sediment. International Journal of Systematic and Evolutionary Microbiology, 63(5), 1602-1609.

Takeuchi, M., Hamana, K., \& Hiraishi, A. (2001). Proposal of the genus Sphingomonas sensu stricto and three new genera, Sphingobium, Novosphingobium and Sphingopyxis, on the basis of phylogenetic and chemotaxonomic analyses. International Journal of Systematic and Evolutionary Microbiology, 51(4), 1405-1417.

Taricska, J. R., Long, D. A., Chen, J. P., Hung, Y.-T., \& Zou, S.-W. (2007). Anaerobic Digestion. In L. K. Wang, \& N. K. Shammas (Eds.), Handbook of environmental engineering biosolids treatment processes (pp. 135-176). Totowa, New Jersey: Humana Press Inc.

Thabet, O. B., Fardeau, M.-L., Suarez-Nuñez, C., Hamdi, M., Thomas, P., Ollivier, B., \& Alazard, D. (2007). Desulfovibrio marinus sp. nov., a moderately halophilic sulfate-reducing bacterium isolated from marine sediments in Tunisia. International Journal of Systematic and Evolutionary Microbiology, 57(9), 2167-2170.

Traversi, D., Villa, S., Lorenzi, E., Degan, R., \& Gilli, G. (2012). Application of a real-time qPCR method to measure the methanogen concentration during anaerobic digestion as an indicator of biogas production capacity. Journal of Environmental Management, 111, 173177.

Ünşar, E., Çığgın, A., Erdem, A., \& Perendeci, N. (2016). Long and short term impacts of CuO, $\mathrm{Ag}$ and $\mathrm{CeO} 2$ nanoparticles on anaerobic digestion of municipal waste activated sludge. Environmental Science: Processes \& Impacts, 18(2), 277-288.

US Research Nanomaterials, I. (2017). US Research Nanomaterials Inc. Retrieved December 5, 2017, from https://www.us-nano.com/inc/sdetail/602

Vandamme, P., Vancanneyt, M., Pot, B., Mels, L., Hoste, B., Dewettinck, D., . . Kersters, K. (1992). Polyphasic taxonomic study of the emended genus Arcobacter with Arcobacter butzleri comb. nov. and Arcobacter skirrowii sp. nov., an aerotolerant bacterium isolated from veterinary specimens. International Journal of Systematic and Evolutionary Microbiology, 42(3), 344-356.

Veldkamp, H. (1960). Isolation and characteristics ofTreponema zuelzerae nov. spec., an anaerobic, free-living spirochete. Antonie van Leeuwenhoek, 26(1), 103-125. 
Venkiteshwaran, K., Bocher, B., Maki, J., \& Zitomer, D. (2015). Relating anaerobic digestion microbial community and process function: supplementary issue: water microbiology. Microbiology Insights, 8(S2), 37-44.

Wang, D., \& Chen, Y. (2016). Critical review of the influences of nanoparticles on biological wastewater treatment and sludge digestion. Critical Reviews in Biotechnology, 35(5), 816828.

Wiegel, J., Tanner, R., \& Rainey, F. (2006). An introduction to the family Clostridiaceae. (pp. 654678). New York, NY.: The prokaryotes.

Xiu, Z.-m., Zhang, Q.-b., Puppala, H. L., Colvin, V. L., \& Alvarez, P. J. (2012). Negligible particlespecific antibacterial activity of silver nanoparticles. Nano Letters, 12(8), 4271-4275.

Xue, Y., Xu, Y., Liu, Y., Ma, Y., \& Zhou, P. (2001). Thermoanaerobacter tengcongensis sp. nov., a novel anaerobic, saccharolytic, thermophilic bacterium isolated from a hot spring in Tengcong, China. International Journal of Systematic and Evolutionary Microbiology, 51(4), 1335-1341.

Yang, Y. (2012a). Impact of metallic nanoparticles on anaerobic digestion. Doctoral dissertation, University of Missouri--Columbia.

Yang, Y., Chen, Q., Wall, J., \& Hu, Z. (2012). Potential nanosilver impact on anaerobic digestion at moderate silver concentrations. Water Research, 46, 1176-1184.

Yang, Y., Yu, K., Xia, Y., Lau, F. T., Tang, D. T., Fung, W. C., .. . Zhang, T. (2014). Metagenomic analysis of sludge from full-scale anaerobic digesters operated in municipal wastewater treatment plants. Applied Microbiology and Biotechnology, 98(12), 5709-5718.

Yutin, N., \& Galperin, M. Y. (2013). A genomic update on clostridial phylogeny: G ram-negative spore formers and other misplaced clostridia. Environmental Microbiology, 15(10), 26312641.

Zhang, D., Trzcinski, A. P., Oh, H.-S., Chew, E., Tan, S. K., Ng, W. J., \& Liu, Y. (2017). Comparison and distribution of copper oxide nanoparticles and copper ions in activated sludge reactors. Journal of Environmental Science and Health, Part A 52(6), 507-514. 


\section{Supplementary data}

\section{DNA quantification}

Bacterial community was studies based on analyses of the total genomic DNA of the sludge in each BMP reactor. Figure S-1 shows the concentration of extracted DNA before performing any analyses. The extracted DNA quantities do not seem to be different among all BMP reactors indicating that AgNPs or CuONPs didn't affect the quantity of the extracted DNA.
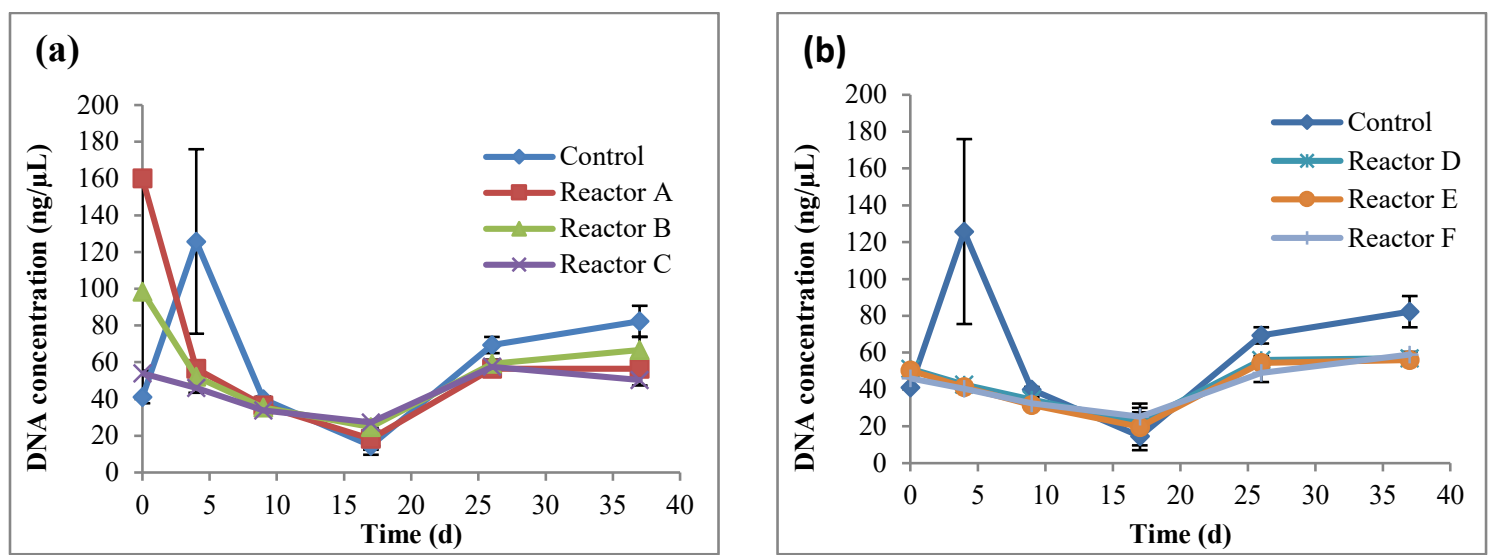

Figure S-1 Total genomic DNA over time, for the control, and reactors A, B, and C (a), and reactors $\mathrm{D}, \mathrm{E}$, and $\mathrm{F}$ (b) (the control had no nanoparticles and reactors $\mathrm{A}, \mathrm{B}$, and $\mathrm{C}$ had $\mathbf{2 , 1 0}$, and $30 \mathrm{mg} \mathrm{AgNPs} / \mathrm{g}$ TS, respectively, and reactors D, E, and F had 2, 10, and $30 \mathrm{mg} \mathrm{CuONPs/g}$ TS, respectively). 
Anaerobic digestion performance

tCOD and sCOD removal
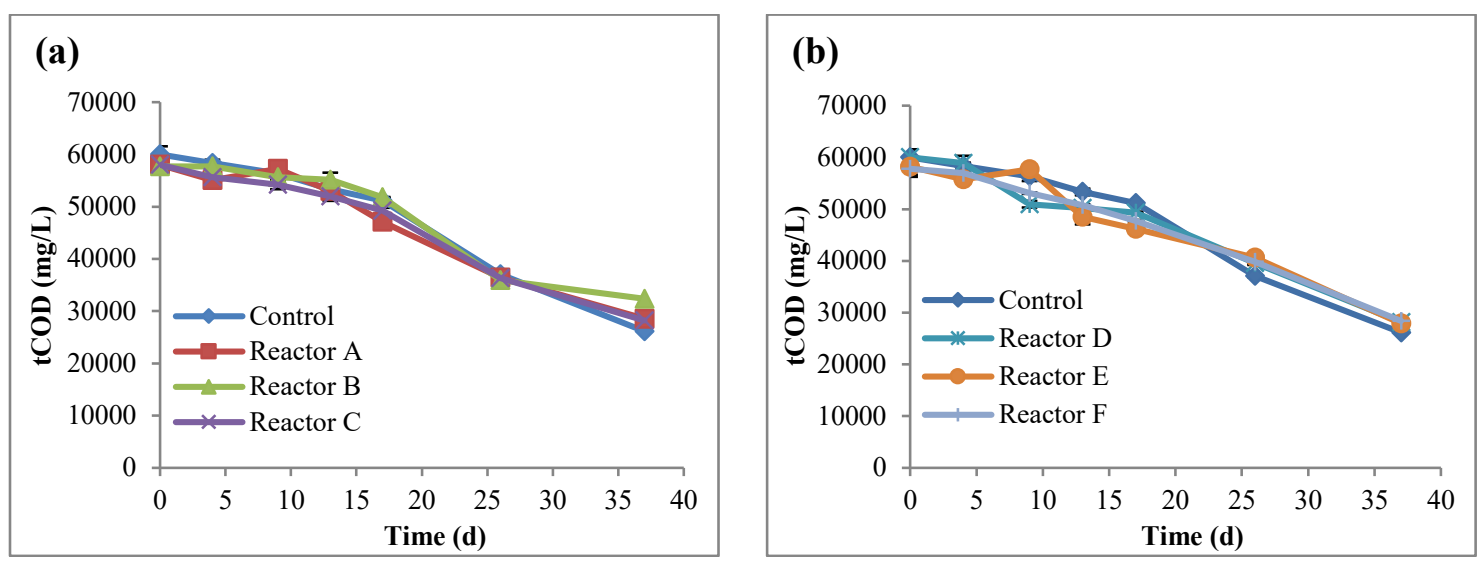

Figure S-2 Concentrations of tCOD over time, for the control and reactors $A, B$, and $C$ (a), and reactors $\mathrm{D}, \mathrm{E}$, and $\mathrm{F}$ (the control had no nanoparticles and reactors $\mathrm{A}, \mathrm{B}$, and $\mathrm{C}$ had 2 , 10, and $30 \mathrm{mg}$ AgNPs/g TS, respectively, and reactors $\mathrm{D}$, E, and F had 2, 10, and $30 \mathrm{mg}$ CuONPs/g TS, respectively).
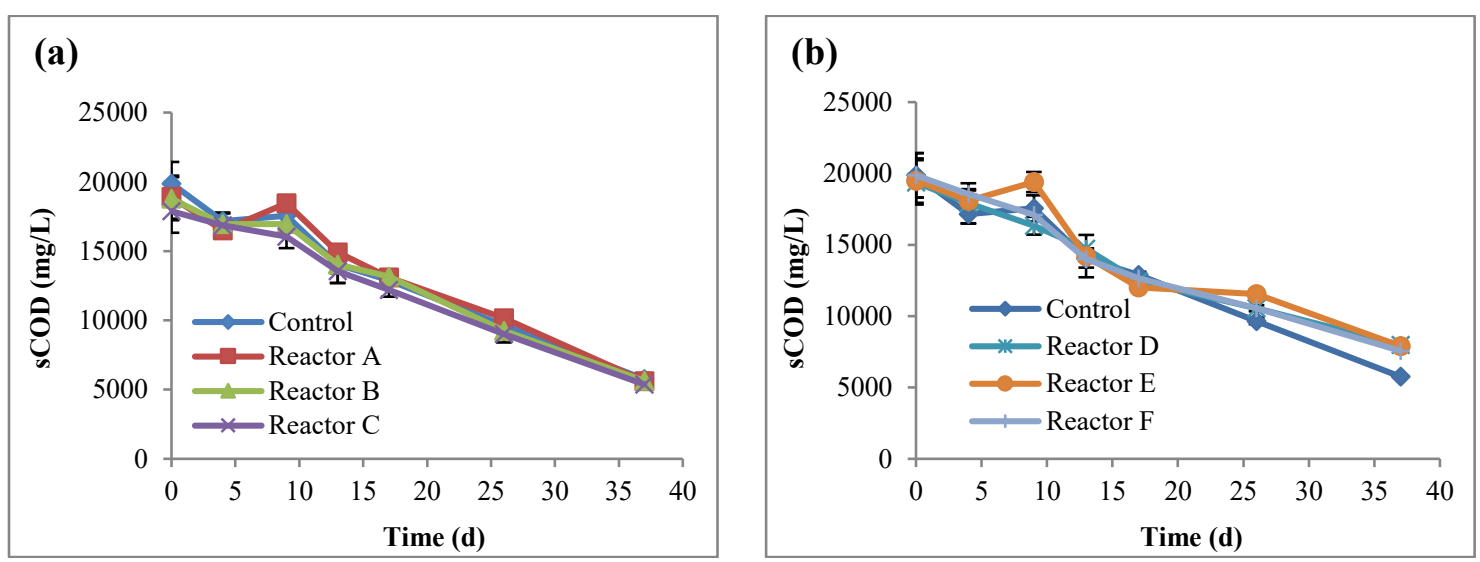

Figure S-3 Concentrations of SCOD over time, for the control and reactors $A$, $B$, and $C$ (a), and reactors $D, E$, and $F$ (b) (the control had no nanoparticles and reactors $A, B$, and $C$ had 2, 10, and $30 \mathrm{mg} \mathrm{AgNPs/g} \mathrm{TS,} \mathrm{respectively,} \mathrm{and} \mathrm{reactors} \mathrm{D,} \mathrm{E,} \mathrm{and} \mathrm{F} \mathrm{had} \mathrm{2,} \mathrm{10,} \mathrm{and} 30 \mathrm{mg}$ CuONPs/g TS, respectively). 
TS and VS removal
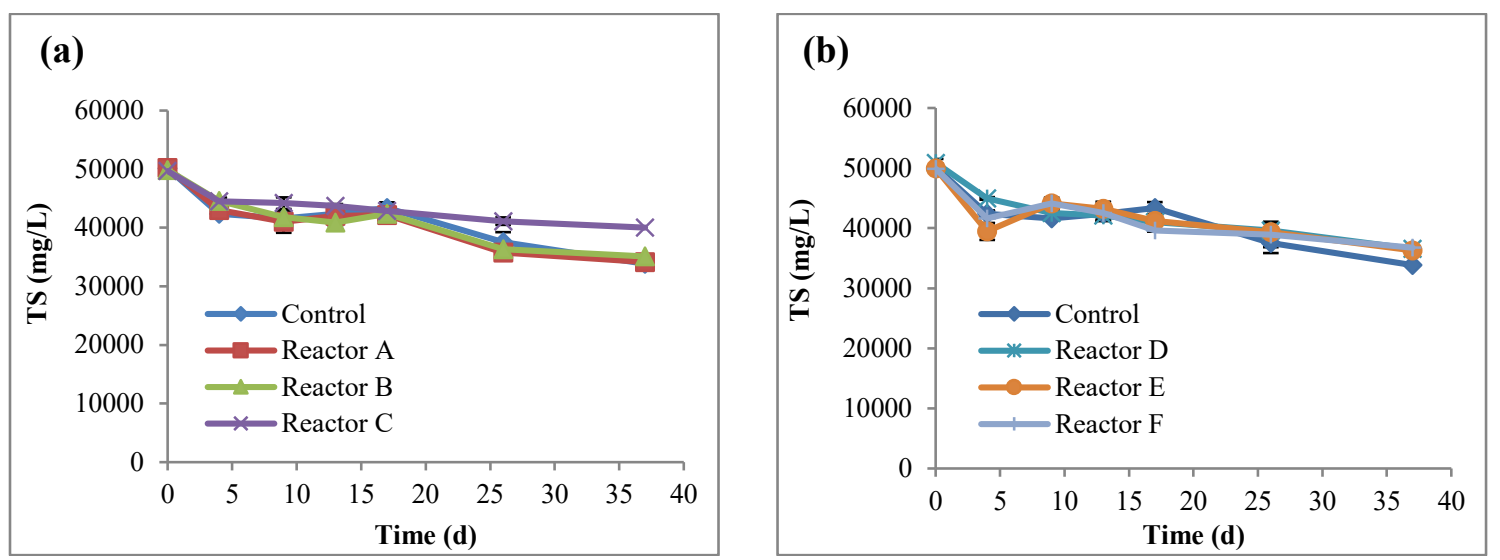

Figure S-4 Concentrations of TS over time, for the control and reactors $A, B$, and $C$ (a), and reactors $\mathrm{D}, \mathrm{E}$, and $\mathrm{F}$ (b) (the control had no nanoparticles and reactors $\mathrm{A}, \mathrm{B}$, and $\mathrm{C}$ had 2,10 , and $30 \mathrm{mg} \mathrm{AgNPs} / \mathrm{g} \mathrm{TS}$, respectively, and reactors $\mathrm{D}$, E, and $\mathrm{F}$ had 2, 10, and $30 \mathrm{mg} \mathrm{CuONPs} / \mathrm{g}$ TS, respectively).
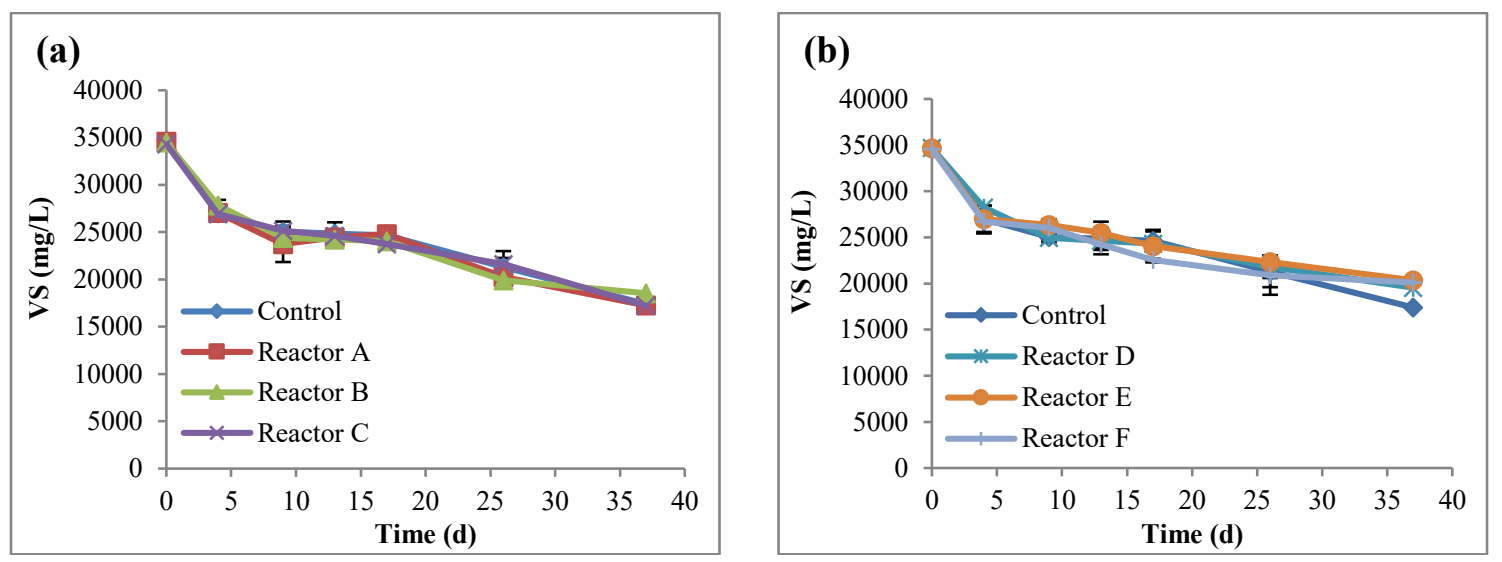

Figure S-5 Concentrations of VS over time, for the control and reactors $A, B$, and $C$ (a), and reactors $\mathrm{D}, \mathrm{E}$, and $\mathrm{F}$ (b) (the control had no nanoparticles and reactors $\mathrm{A}, \mathrm{B}$, and $\mathrm{C}$ had 2, 10, and $30 \mathrm{mg} \mathrm{AgNPs} / \mathrm{g} \mathrm{TS}$, respectively, and reactors $\mathrm{D}$, E, and $\mathrm{F}$ had 2, 10, and $30 \mathrm{mg} \mathrm{CuONPs} / \mathrm{g}$ TS, respectively). 


\section{Live/Dead images}
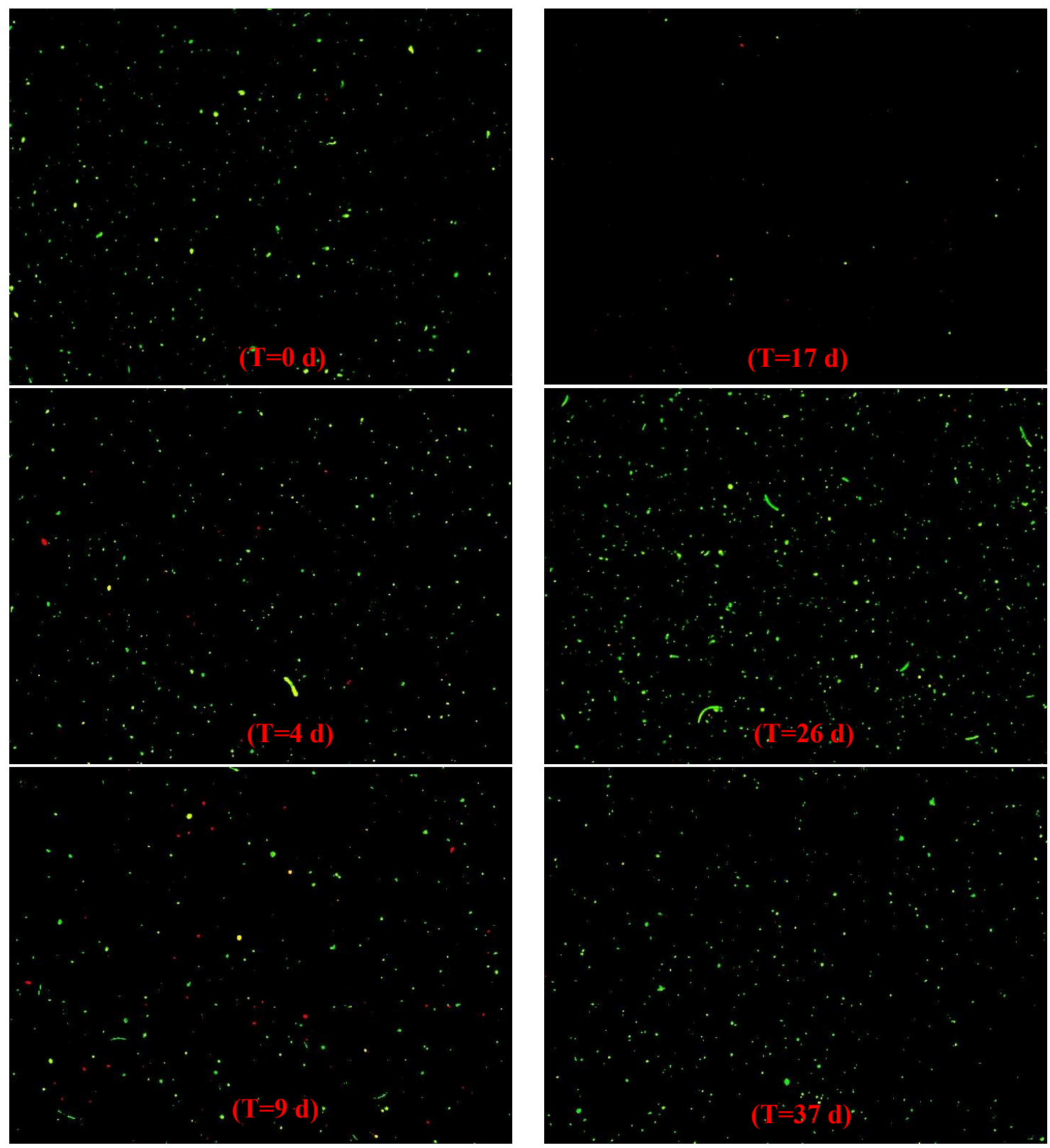

Figure S-6 Live/Dead cells images for the control over time. 

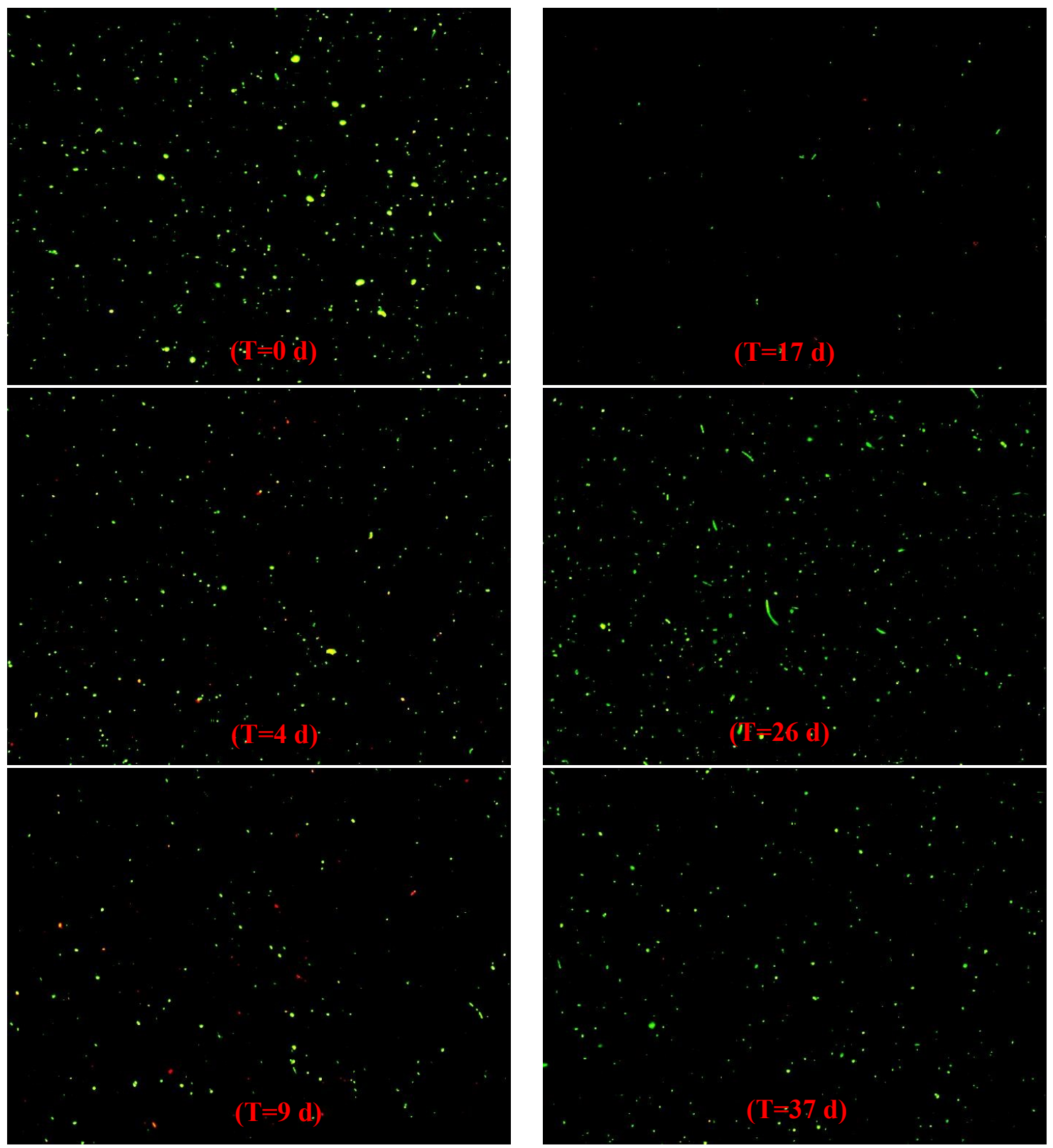

Figure S-7 Live/Dead cells images for reactor A ( 2 mg AgNPs/g TS of sludge). 

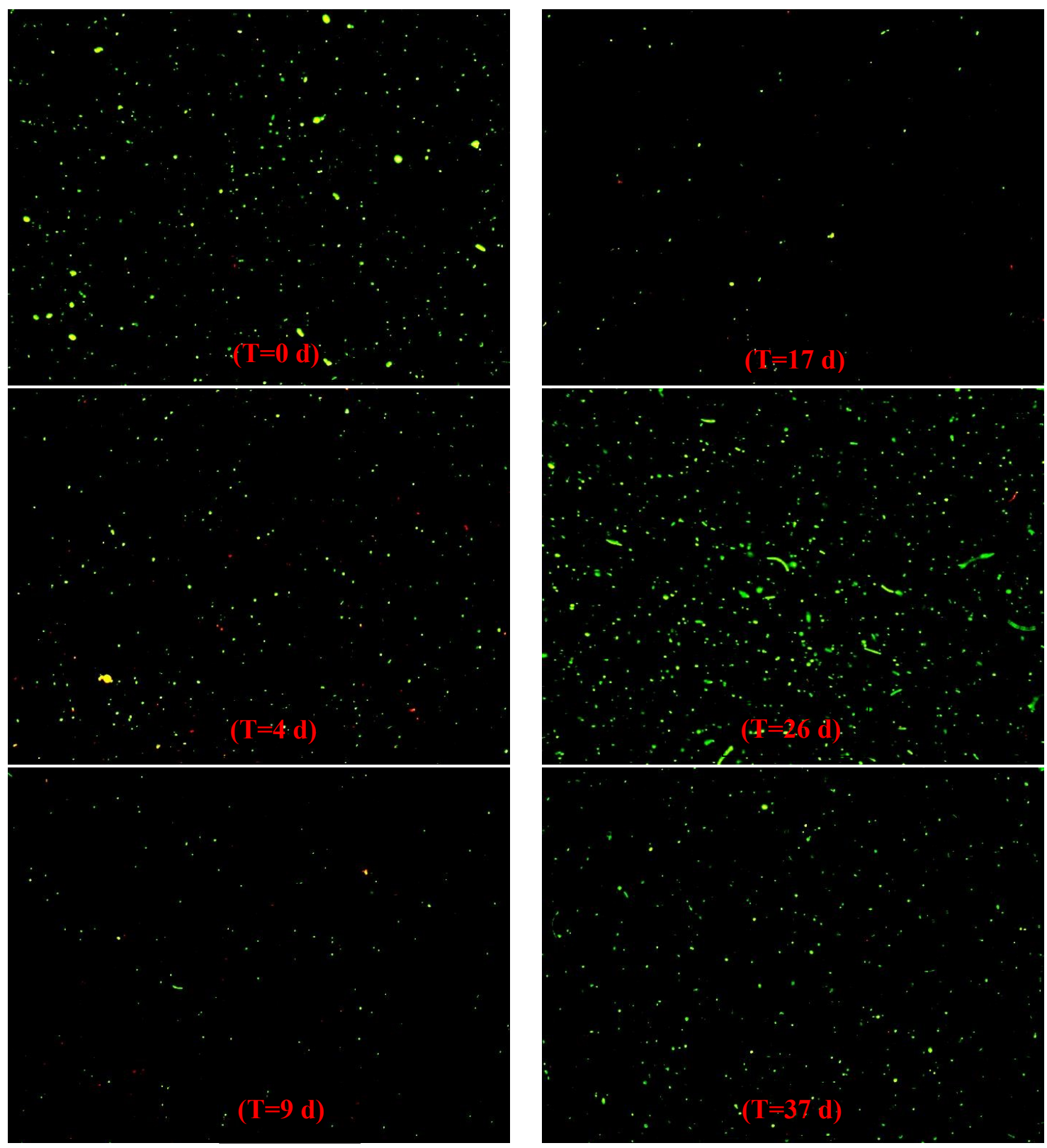

Figure S-8 Live/Dead cells images for reactor B (10 mg AgNPs/g TS of sludge). 


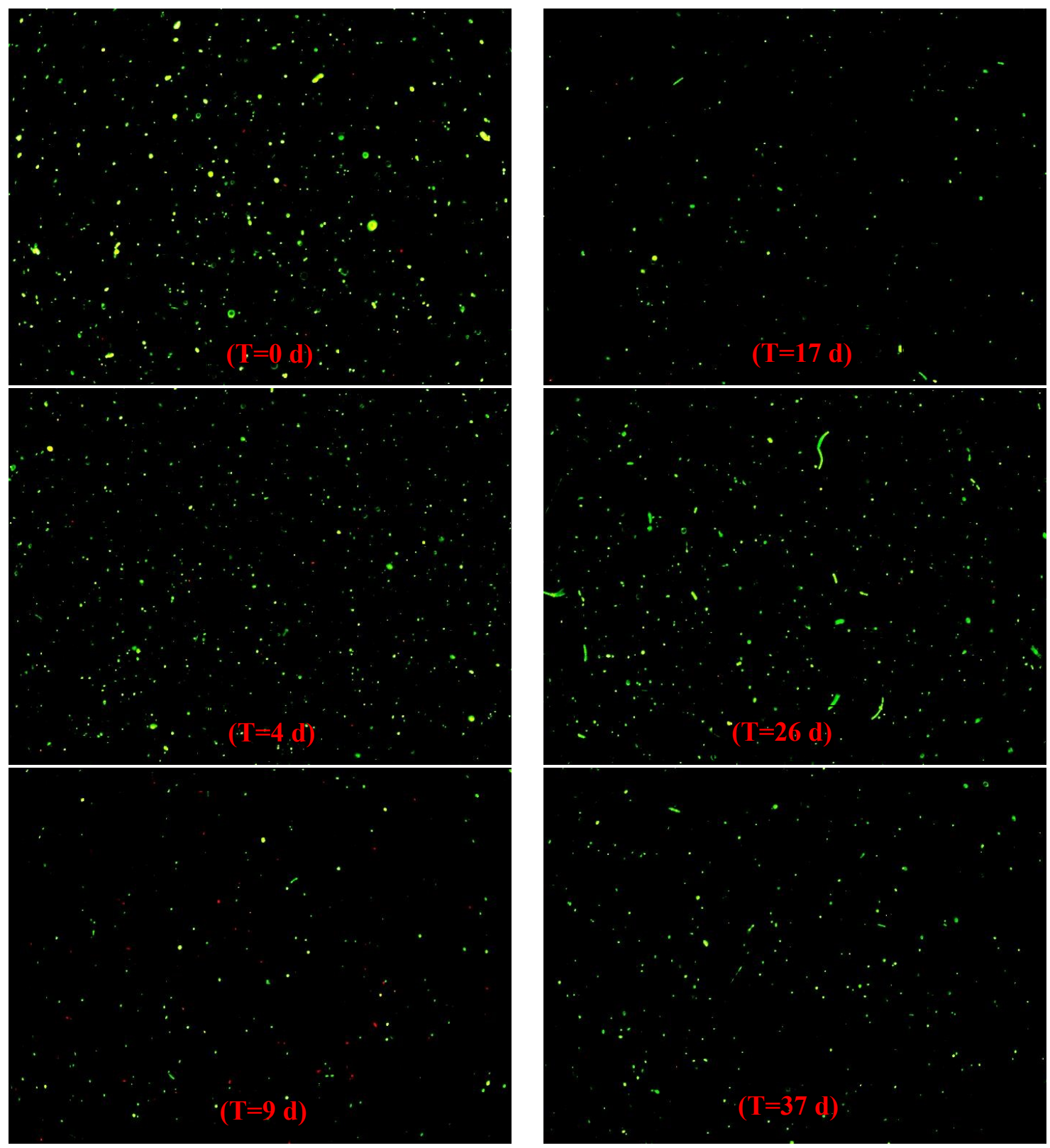

Figure S-9 Live/Dead cells images for reactor C (30 mg AgNPs/ g TS of sludge). 

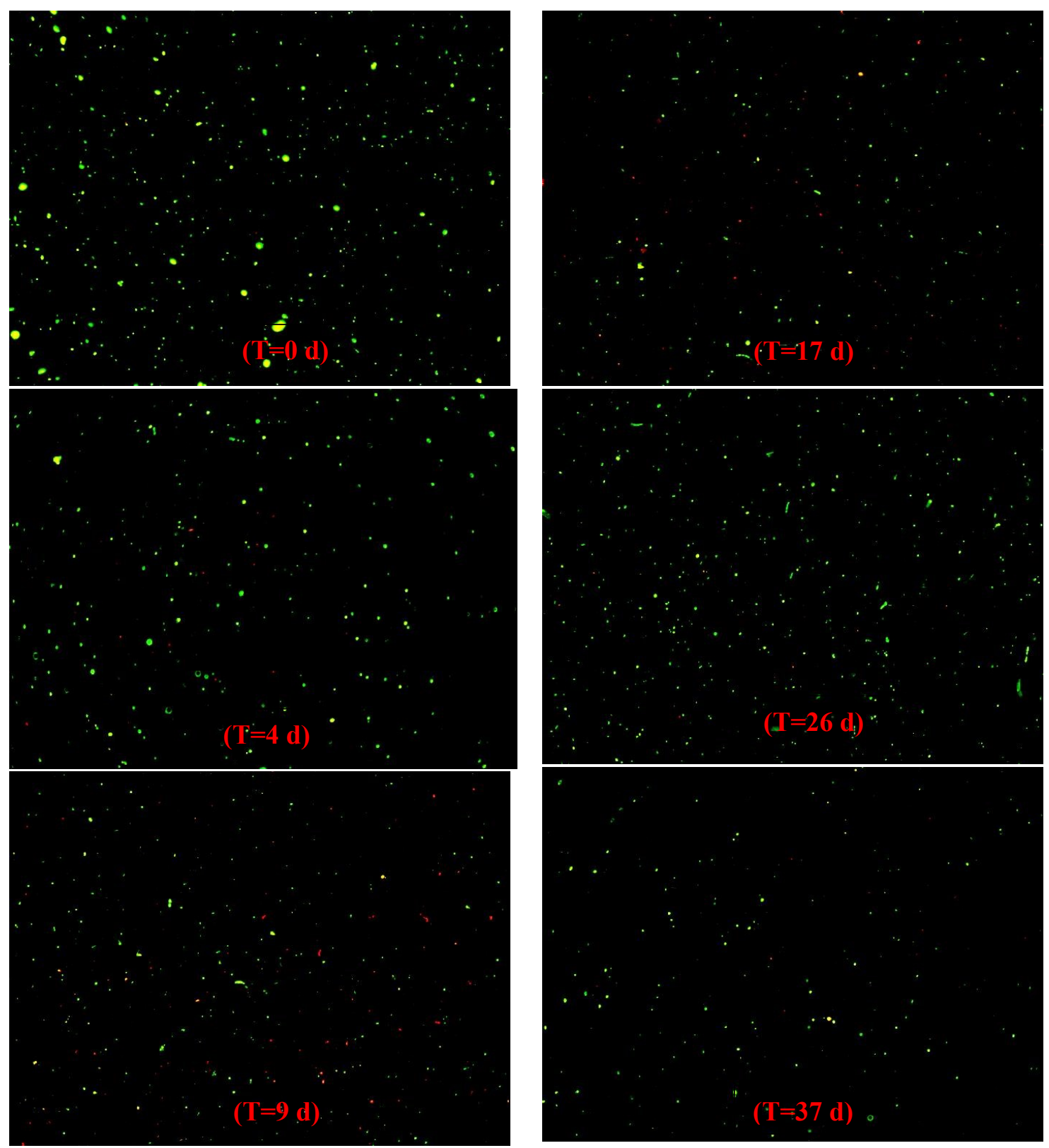

Figure S-10 Live/Dead cells images for reactor D (2 mg AgNPs/ g TS of sludge). 

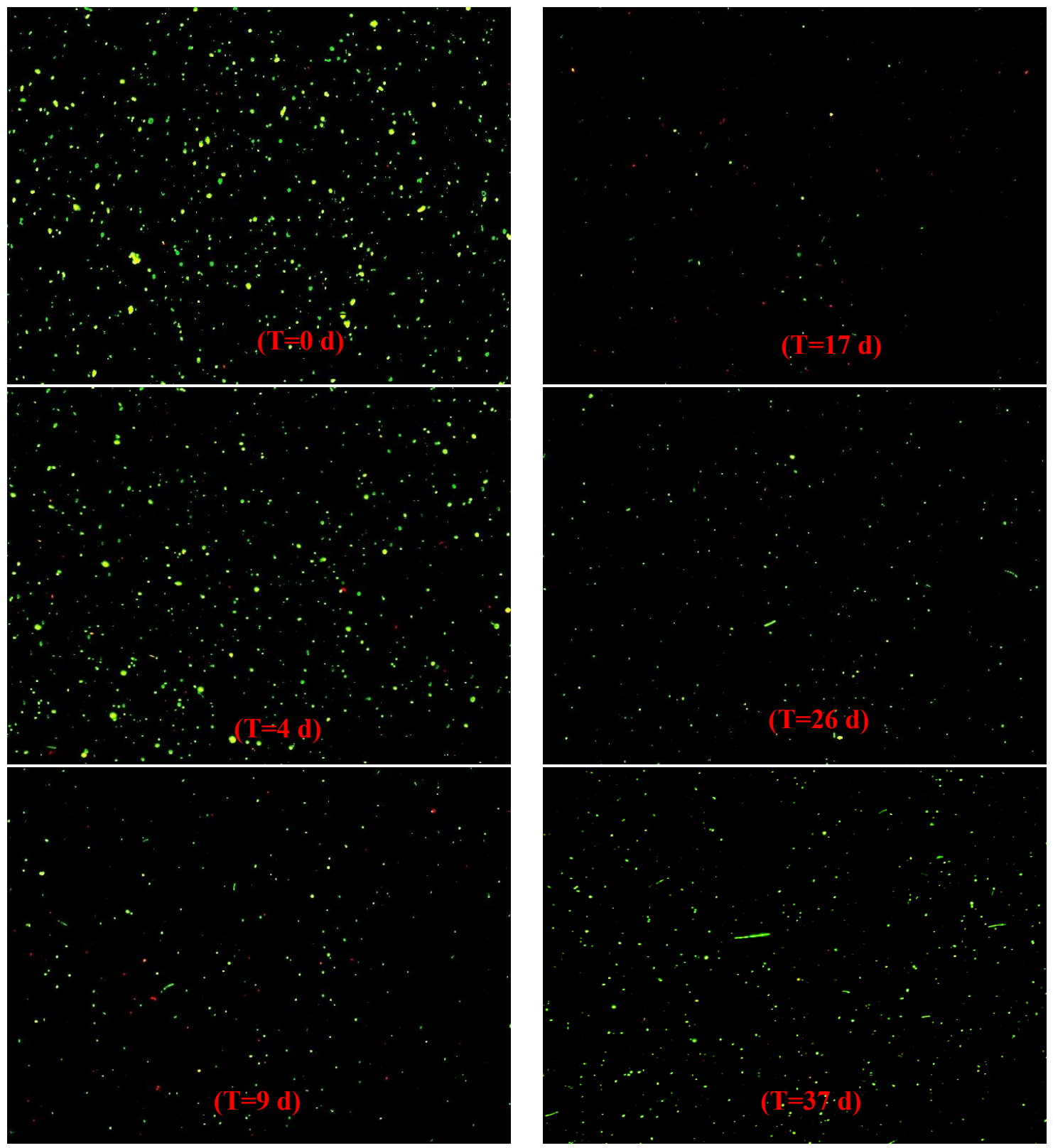

Figure S-11 Live/Dead cells images for reactor E (10 mg AgNPs/ g TS of sludge). 


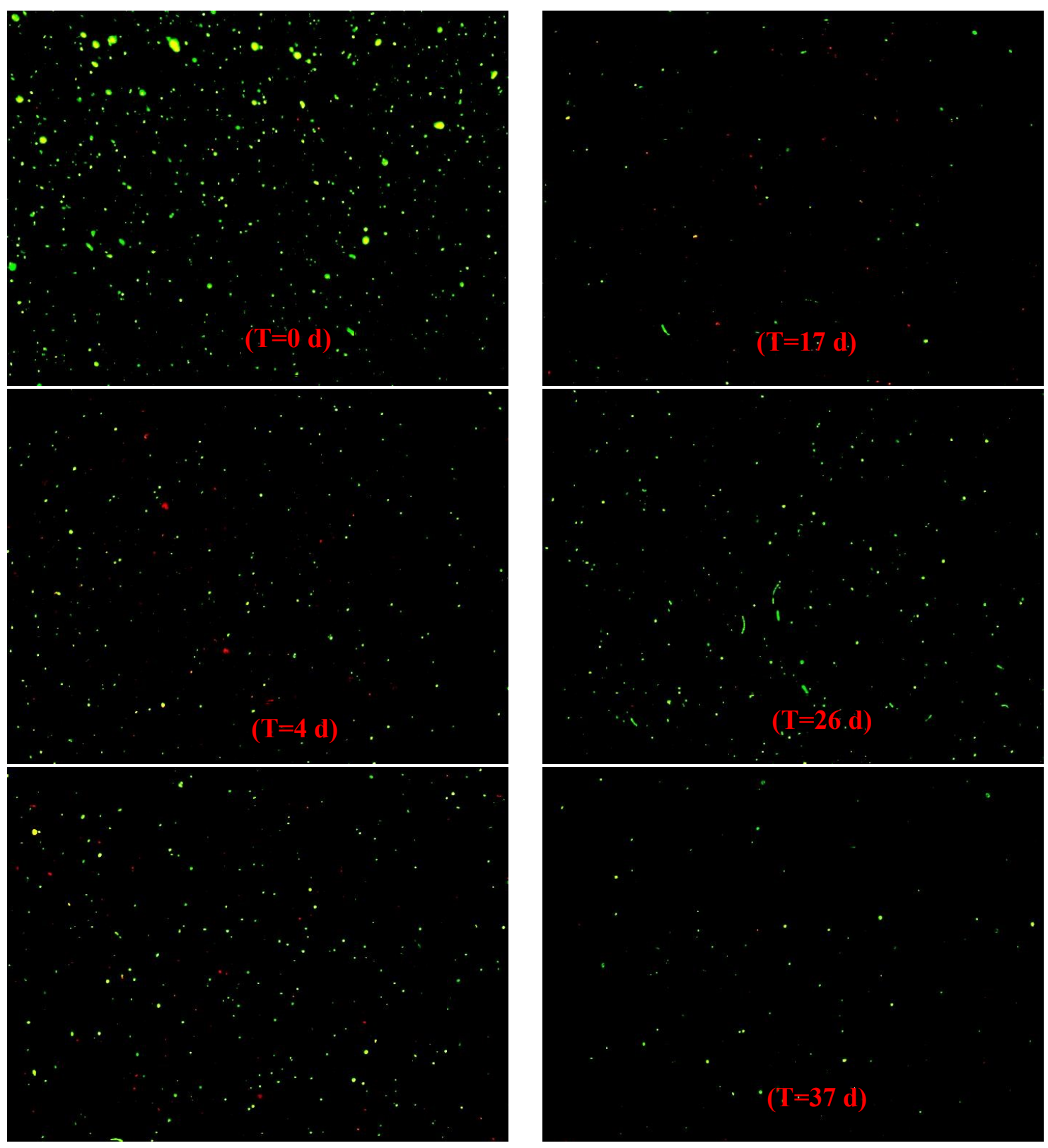

Figure S-12 Live/Dead cells images for reactor F (30 mg AgNPs/ g TS of sludge). 


\section{Genus of interest and their important role in the sludge anaerobic digestion}

\section{Table S-1 Genus of interest and their role in the sludge anaerobic digestion process}

\begin{tabular}{|c|c|c|c|c|}
\hline Genus & Specification & Function in AD & Action & Reference \\
\hline Acidovorax & $\begin{array}{l}\text { Gram-negative, motile } \\
\text { or non-motile } \\
\text { Aerobic/facultative } \\
\text { anaerobic. }\end{array}$ & $\begin{array}{l}\text { Utilize acetate for denitrification in } \\
\text { activated sludge, nitrate reduction and } \\
\text { fatty acids, sugars and proteins/amino } \\
\text { acids assimilation. }\end{array}$ & $\begin{array}{l}\text { Hydrolysis and } \\
\text { fermentation. }\end{array}$ & $\begin{array}{l}\text { (Schulze et al., } \\
\text { 1999; Ginige et } \\
\text { al., 2005) }\end{array}$ \\
\hline Arcobacter & $\begin{array}{l}\text { Gram-negative, motile, } \\
\text { aerotolerance. }\end{array}$ & $\begin{array}{l}\text { Denitrification and } \mathrm{H}_{2} \mathrm{~S} \text {-producing } \\
\text { bacteria. Assimilation of organic, amino } \\
\text { acids, fatty acids, sugars and } \\
\text { proteins/amino acids. }\end{array}$ & $\begin{array}{l}\text { Hydrolysis and } \\
\text { fermentation. }\end{array}$ & $\begin{array}{l}\text { (Vandamme et } \\
\text { al., 1992; } \\
\text { Roalkvam et al., } \\
\text { 2015) }\end{array}$ \\
\hline Novosphingobium & $\begin{array}{l}\text { Gram-negative, non- } \\
\text { motile, facultative } \\
\text { anaerobic. }\end{array}$ & $\begin{array}{l}\text { Degrading a number of organic } \\
\text { compounds, including aromatic } \\
\text { hydrocarbons, polycyclic aromatic } \\
\text { hydrocarbons (PAH) } \\
\text { polychlorophenols and carbohydrates. } \\
\text { Assimilate fatty acids, sugars and } \\
\text { proteins/amino acids. }\end{array}$ & Hydrolysis. & $\begin{array}{l}\text { (Takeuchi et al., } \\
\text { 2001) }\end{array}$ \\
\hline Syntophus & $\begin{array}{l}\text { Gram-negative, } \\
\text { obligate anaerobes. }\end{array}$ & $\begin{array}{l}\text { Fermentation and degradation of fatty } \\
\text { acids and benzoate in syntrophic } \\
\text { association with hydrogen-using } \\
\text { microorganisms. }\end{array}$ & Acetogenesis. & $\begin{array}{l}\text { (Elshahed \& } \\
\text { McInerney, } \\
\text { 2001) }\end{array}$ \\
\hline Sedimentibacter & $\begin{array}{l}\text { Gram-negative, } \\
\text { obligate anaerobes. }\end{array}$ & $\begin{array}{l}\text { Fermentation and degradation of fatty } \\
\text { acids, amino acids and pyruvate proteins. } \\
\text { Sugars are not fermented by members of } \\
\text { the genus. }\end{array}$ & $\begin{array}{l}\text { Fermentation } \\
\text { and } \\
\text { acetogenesis. }\end{array}$ & $\begin{array}{l}\text { (Breitenstein et } \\
\text { al., 2002) }\end{array}$ \\
\hline Thauera & $\begin{array}{l}\text { Gram-negative, motile. } \\
\text { Facultative anaerobes }\end{array}$ & $\begin{array}{l}\text { Denitrification. Utilizing aromatic } \\
\text { compounds, amino acids and organic } \\
\text { substrates such as sugars, acetate, lactate, } \\
\text { pyruvate and ethanol, while using nitrate, } \\
\text { nitrite or oxygen as electron acceptor. }\end{array}$ & $\begin{array}{l}\text { Hydrolysis and } \\
\text { fermentation. }\end{array}$ & $\begin{array}{l}\text { (Garrity et al., } \\
\text { 2005) }\end{array}$ \\
\hline Oscillibacter & $\begin{array}{l}\text { Gram-negative, } \\
\text { obligate anaerobes and } \\
\text { motile. }\end{array}$ & $\begin{array}{l}\text { Fermentation. } \\
\text { Assimilate sugars. }\end{array}$ & $\begin{array}{l}\text { Hydrolysis and } \\
\text { fermentation. }\end{array}$ & $\begin{array}{l}\text { (Iino et al., } \\
\text { 2007) }\end{array}$ \\
\hline Ruminiclostridium & $\begin{array}{l}\text { Gram-positive, } \\
\text { anaerobic organism }\end{array}$ & $\begin{array}{l}\text { Fermentation of sugars derived from } \\
\text { cellulose and production of extracellular } \\
\text { multi-enzymatic complexes called } \\
\text { cellulosomes, which efficiently degrade } \\
\text { the crystalline cellulose. }\end{array}$ & $\begin{array}{l}\text { Hydrolysis and } \\
\text { fermentation. }\end{array}$ & $\begin{array}{l}\text { (Fosses et al., } \\
\text { 2017) }\end{array}$ \\
\hline Simplicispira & Facultative anaerobes. & $\begin{array}{l}\text { Are able to assimilate fatty acids, sugars } \\
\text { and proteins/amino acids }\end{array}$ & $\begin{array}{l}\text { Hydrolysis and } \\
\text { fermentation. }\end{array}$ & $\begin{array}{l}\text { (Grabovich et } \\
\text { al., 2006) }\end{array}$ \\
\hline Clostridium & $\begin{array}{l}\text { Gram-positive, obligate } \\
\text { anaerobes. }\end{array}$ & $\begin{array}{l}\text { Fermentation. Production of acetic acid, } \\
\text { lactic acid and/or ethanol, propanol or } \\
\text { butanol. }\end{array}$ & $\begin{array}{l}\text { Hydrolysis, } \\
\text { fermentation } \\
\text { and } \\
\text { Acetogenesis. }\end{array}$ & $\begin{array}{l}\text { (Wiegel et al., } \\
\text { 2006) }\end{array}$ \\
\hline Trichococcus & $\begin{array}{l}\text { Gram-positive, } \\
\text { facultative anaerobes. }\end{array}$ & Assimilate fatty acids and sugar. & Fermentation. & $\begin{array}{l}\text { (Pikuta et al., } \\
2006)\end{array}$ \\
\hline Bacteroides & $\begin{array}{l}\text { Gram-negative, } \\
\text { obligate anaerobes, } \\
\text { motile or non-motile. }\end{array}$ & $\begin{array}{l}\text { Producing various acids such as lactic } \\
\text { acid, propionic acid, formic acid and } \\
\text { acetic acid from carbohydrates }\end{array}$ & Hydrolysis. & (Li et al., 2013) \\
\hline
\end{tabular}


Table S-1 (Continued).

\begin{tabular}{|c|c|c|c|c|}
\hline Alistipes & $\begin{array}{l}\text { Gram-negative, obligate } \\
\text { anaerobes, motile. }\end{array}$ & $\begin{array}{l}\text { Fermentation. Assimilation of glucose } \\
\text { to produced succinic and acetic acids as } \\
\text { end products. }\end{array}$ & Fermentation. & $\begin{array}{l}\text { (Nagai et al., } \\
2010 \text { ) }\end{array}$ \\
\hline Rhodoferax & $\begin{array}{l}\text { Gram-negative, facultative } \\
\text { anaerobes, motile. }\end{array}$ & $\begin{array}{l}\text { Assimilation of a range of different } \\
\text { carbon sources, including amino acids, } \\
\text { sugars and short chain fatty acids. }\end{array}$ & $\begin{array}{l}\text { Hydrolysis and } \\
\text { fermentation. }\end{array}$ & $\begin{array}{l}\text { (Finneran et } \\
\text { al., 2003) }\end{array}$ \\
\hline Aeromonas & $\begin{array}{l}\text { Gram-negative, facultative } \\
\text { anaerobic. }\end{array}$ & $\begin{array}{l}\text { Fermentation of organic matter and } \\
\text { sugars. }\end{array}$ & $\begin{array}{l}\text { Hydrolysis and } \\
\text { fermentation. }\end{array}$ & $\begin{array}{l}\text { (Abbott et al., } \\
2003)\end{array}$ \\
\hline Desulfotomaculum & $\begin{array}{l}\text { Gram-positive, obligate } \\
\text { anaerobes. }\end{array}$ & $\begin{array}{l}\text { Sulfate-reducing bacteria } \\
\text { utilizing sulfate }\left(\mathrm{SO}_{4}^{2-}\right) \text { as } \\
\text { terminal electron acceptor, reducing it } \\
\text { to hydrogen sulfide }\left(\mathrm{H}_{2} \mathrm{~S}\right) \text {. Nitrogen } \\
\text { fixation }\end{array}$ & Acetogenesis. & $\begin{array}{l}\text { (Aullo et al., } \\
\text { 2013) }\end{array}$ \\
\hline Lachnoclostridium & $\begin{array}{l}\text { Gram-positive, obligate } \\
\text { anaerobes. }\end{array}$ & $\begin{array}{l}\text { Fermentative. Assimilate sugars. } \\
\text { Acetate is the major end product of } \\
\text { mono- and disaccharide fermentation. }\end{array}$ & Fermentation. & $\begin{array}{l}\text { (Yutin \& } \\
\text { Galperin, } \\
\text { 2013) }\end{array}$ \\
\hline Hydrogenophaga & $\begin{array}{l}\text { Gram-negative, anaerobic, } \\
\text { facultative aerobic, motile. }\end{array}$ & $\begin{array}{l}\text { Enhanced biological phosphorus } \\
\text { removal. Denitrification, nitrate } \\
\text { reduction using the oxidation of } \mathrm{H}_{2} \text { as } \\
\text { an energy source and } \mathrm{CO}_{2} \text { as a carbon } \\
\text { source. }\end{array}$ & & $\begin{array}{l}\text { (Chung et al., } \\
\text { 2007) }\end{array}$ \\
\hline Gracilibacter & $\begin{array}{l}\text { Gram-negative, } \\
\text { obligatorily anaerobic. }\end{array}$ & $\begin{array}{l}\text { Fermentation. Acetate, lactate and } \\
\text { ethanol are the main fermentation end } \\
\text { products of glucose. }\end{array}$ & Fermentation. & $\begin{array}{l}\text { (Lee et al., } \\
\text { 2006) }\end{array}$ \\
\hline Christensenella & $\begin{array}{l}\text { Gram-negative, anaerobic, } \\
\text { nonmotile. }\end{array}$ & $\begin{array}{l}\text { Fermentation. Acetic acid and a small } \\
\text { amount of butyric acid the end products } \\
\text { of glucose fermentation. }\end{array}$ & Fermentation. & $\begin{array}{l}\text { (Morotomi et } \\
\text { al., 2012) }\end{array}$ \\
\hline Moorella & Gram-positive, anaerobic. & $\begin{array}{l}\text { Production of acetate by reducing } \\
\text { carbon dioxide. }\end{array}$ & Acetogenesis. & $\begin{array}{l}\text { (Islam et al., } \\
2015)\end{array}$ \\
\hline Acidaminococcus & Gram-negative, anaerobes. & Assimilation of amino acids. & Fermentation. & $\begin{array}{l}\text { (Euzéby, } \\
1997)\end{array}$ \\
\hline Sunxiuqinia & $\begin{array}{l}\text { Gram-negative, facultative } \\
\text { anaerobic, nonmotile. }\end{array}$ & $\begin{array}{l}\text { Assimilation of yeast extract, tryptone, } \\
\text { casein and casamino acids with } \\
\text { fermentation. }\end{array}$ & Hydrolysis. & $\begin{array}{l}\text { (Takai et al., } \\
\text { 2013) }\end{array}$ \\
\hline Desulfovibrio & $\begin{array}{l}\text { Gram-negative, } \\
\text { obligate anaerobic, motile. }\end{array}$ & Fermentation. Sulfate-reducing bacteria. & Fermentation. & $\begin{array}{l}\text { (Thabet et al., } \\
\text { 2007) }\end{array}$ \\
\hline Syntrophomonas & $\begin{array}{l}\text { Gram-negative, anaerobe, } \\
\text { non-motile. }\end{array}$ & $\begin{array}{l}\text { Fatty-acid-using bacterium, acetogen } \\
\text { that require syntrophic interaction with } \\
\mathrm{H} \text {,-utilizing bacteria. }\end{array}$ & Acetogenesis. & $\begin{array}{l}\text { (Hatamoto et } \\
\text { al., 2007) }\end{array}$ \\
\hline Ruminococcus & $\begin{array}{l}\text { Gram-positive bacterium, } \\
\text { obligate anaerobic, non- } \\
\text { motile. }\end{array}$ & $\begin{array}{l}\text { Acetate and succinate are the major end } \\
\text { products of cellulose and cellobiose } \\
\text { fermentation. }\end{array}$ & $\begin{array}{l}\text { Fermentation } \\
\text { and } \\
\text { acetogenesis. }\end{array}$ & $\begin{array}{l}\text { (Ben David et } \\
\text { al., 2015) }\end{array}$ \\
\hline Treponema & $\begin{array}{l}\text { Gram-negative, anaerobic, } \\
\text { motile. }\end{array}$ & $\begin{array}{l}\text { End products of glucose fermentation } \\
\text { are: lactic, acetic, and succinic acids, } \\
\mathrm{CO}_{2} \text {, and } \mathrm{H} \text {. }\end{array}$ & Fermentation. & $\begin{array}{l}\text { (Veldkamp } \\
1960)\end{array}$ \\
\hline Caldicoprobacter & $\begin{array}{l}\text { Gram-positive, obligate } \\
\text { anaerobic. }\end{array}$ & Fermentation. Assimilation of sugars. & Fermentation. & $\begin{array}{l}\text { (Bouanane- } \\
\text { Darenfed et } \\
\text { al., 2015) }\end{array}$ \\
\hline Faecalicatena & $\begin{array}{l}\text { Gram-positive, obligate } \\
\text { anaerobe motile. }\end{array}$ & $\begin{array}{l}\text { Assimilation of sugars. Production of } \\
\text { hydrogen sulfide. }\end{array}$ & $\begin{array}{l}\text { Hydrolysis and } \\
\text { fermentation. }\end{array}$ & $\begin{array}{l}\text { (Sakamoto et } \\
\text { al., 2017) }\end{array}$ \\
\hline Geobacter & $\begin{array}{l}\text { Gram-negative, obligate } \\
\text { anaerobe, motile. }\end{array}$ & Decomposition of organic compounds. & $\begin{array}{l}\text { Hydrolysis and } \\
\text { fermentation. }\end{array}$ & $\begin{array}{l}\text { (Caccavo et } \\
\text { al., 1994) }\end{array}$ \\
\hline Prolixibacter & $\begin{array}{l}\text { Gram-negative, facultative } \\
\text { anaerobe, non-motile. }\end{array}$ & Fermentation of sugars. & Fermentation. & $\begin{array}{l}\text { (Holmes et al., } \\
\text { 2007) }\end{array}$ \\
\hline
\end{tabular}


Table S-1 (Continued).

\begin{tabular}{|c|c|c|c|c|}
\hline Sphaerochaeta & $\begin{array}{l}\text { Gram-negative, } \\
\text { anaerobe, non-motile. }\end{array}$ & $\begin{array}{l}\text { Fermentation. Assimilation of } \\
\text { fructose, galactose, glucose, mannose, } \\
\text { raffinose, sucrose, xylose. Ethanol, } \\
\text { acetate and formate are major end } \\
\text { products. }\end{array}$ & $\begin{array}{l}\text { Hydrolysis and } \\
\text { fermentation. }\end{array}$ & $\begin{array}{l}\text { (Ritalahti et } \\
\text { al., 2012) }\end{array}$ \\
\hline Romboutsia & $\begin{array}{l}\text { Gram-positive, obligate } \\
\text { anaerobic, non-motile. }\end{array}$ & $\begin{array}{l}\text { Fermentation of sugars, including } \\
\text { glucose. Major end products are } \\
\text { acetate, formate and sometimes } \\
\text { lactate. }\end{array}$ & $\begin{array}{l}\text { Hydrolysis, } \\
\text { fermentation and } \\
\text { acetogenesis. }\end{array}$ & $\begin{array}{l}\text { (Gerritsen et } \\
\text { al., 2015) }\end{array}$ \\
\hline Microbacter & $\begin{array}{l}\text { Gram- } \\
\text { negative, anaerobic, } \\
\text { non-motile. }\end{array}$ & $\begin{array}{l}\text { utilized yeast extract and various } \\
\text { sugars as substrates and formed } \\
\text { propionate, lactate and acetate as } \\
\text { major fermentation products }\end{array}$ & Fermentation. & $\begin{array}{l}\text { (Sanchez- } \\
\text { Andrea et al. } \\
\text { 2014) }\end{array}$ \\
\hline Thermoanaerobacter & $\begin{array}{l}\text { Gram-negative, obligate } \\
\text { anaerobic. }\end{array}$ & $\begin{array}{l}\text { Assimilation of sugars and acetate is } \\
\text { the main end product from glucose } \\
\text { fermentation. }\end{array}$ & $\begin{array}{l}\text { Fermentation and } \\
\text { acetogenesis. }\end{array}$ & $\begin{array}{l}\text { (Xue et al., } \\
\text { 2001) }\end{array}$ \\
\hline Pelotomaculum & $\begin{array}{l}\text { Gram-positive, obligate } \\
\text { anaerobic, non-motile. }\end{array}$ & $\begin{array}{l}\text { Obligate syntrophic bacterium with } \\
\text { methanogens. Anaerobic degradation } \\
\text { of complex organic matter under } \\
\text { methanogenic conditions. }\end{array}$ & Acetogenesis. & $\begin{array}{l}\text { (machi et al., } \\
\text { 2007) }\end{array}$ \\
\hline Syntrophobacter & $\begin{array}{l}\text { Gram-negative, } \\
\text { anaerobic, non-motile. }\end{array}$ & $\begin{array}{l}\text { Degradation of complex organic } \\
\text { matter under methanogenic } \\
\text { conditions. The end products are } \\
\text { acetate and } \mathrm{CO}_{2} \text {. }\end{array}$ & $\begin{array}{l}\text { Hydrolysis, } \\
\text { fermentation and } \\
\text { acetogenesis. }\end{array}$ & $\begin{array}{l}\text { (Chen et al., } \\
\text { 2005) }\end{array}$ \\
\hline Acetobacterium & $\begin{array}{l}\text { Gram-positive, } \\
\text { anaerobic. }\end{array}$ & Acetogen. & Acetogenesis. & $\begin{array}{l}\text { (Balch et al., } \\
1977)\end{array}$ \\
\hline
\end{tabular}




\title{
Chapter 5: Impact and Toxicity of AgNPs and CuONPs on Soil Bacterial Community Structure after Land Application of Anaerobically Digested
} Sludge

\begin{abstract}
Silver nanoparticles (AgNPs) and copper oxide nanoparticles (CuONPs) are used extensively in many industrial antimicrobial applications, which has led to their release and presence in wastewater sludge and, subsequently, in soil due to the wide use of treated sludge (biosolids) as fertilizer. This study simulated the land application of biosolids and examined the impact of two different concentrations of AgNPs and CuONPs on bacterial composition and potential functionality. The nanoparticles were first subjected to an anaerobic digesting process with wastewater sludge, then introduced to soil in accordance with methodology consistent with biosolids land application. The experiment consisted of five soil microcosms, each with $0.01 \mathrm{~g}$ TS sludge/g soil. The control microcosm received sludge without nanoparticles, while the other four microcosms received sludge containing 2 and $30 \mathrm{mg} \mathrm{NPs} / \mathrm{g}$ TS of sludge for each of AgNPs (reactors A and B) and CuONPs (reactors C and D). All microcosms were incubated at room temperature and sampled seven times throughout a 105 days period. Metagenomic bacterial community structure was determined using high-throughput sequencing with the MinION and the EPI2ME Fastq 16S workflow from Oxford Nanopore Technologies. The metagenomic results showed the presence of the four most abundant phyla (relative abundance of $>90 \%$ ) to be: Proteobacteria, Bacteroidetes, Firmicutes and Gemmatimonadetes. Of these, Proteobacteria was most affected by the higher concentration of AgNPs and both
\end{abstract}


concentrations of CuONPs, with lower percentage abundance compared to the control at $8.4,17$, and $19 \%$ for reactors $\mathrm{B}, \mathrm{C}$ and $\mathrm{D}$, respectively. The next most abundant group of phyla consisted of 13 phyla whose total relative abundance compared to the control showed an increase of $38.4,113$, and $205 \%$ in reactors B, C and D, respectively. Investigating the population of bacterial genera representing the plant growth promoting rhizobacteria (PGPR) showed a total population decrease of $10.6,18,41.2$, and $40.3 \%$ compared to the control for reactors A, B, C and D, respectively. Thus, CuONPs exhibited a higher level of toxicity than the AgNPs at both phylum and genus taxonomic levels.

Keywords: Silver nanoparticles, copper oxide nanoparticles, anaerobically digested sludge, land application, bacterial community

\subsection{Introduction}

Silver and copper oxide nanoparticles are currently two of the most widely used in nanotechnology due to their antimicrobial properties. They are incorporated in many applications, including various consumer products, medical device coating and other industrial processes due to their unique properties that facilitate various antimicrobial applications (Colvin, 2003; Reidy et al., 2013; Khodashenas, 2016; Fernando et al., 2018; Kalińska et al., 2019). The growing interest in nanotechnology has led to increased worldwide production and application of nanoparticles, including AgNPs and CuONPs in various industrial and commercial sectors. Thus, their increasing production and use have raised considerable environmental and public health concerns (Turco et al., 2011; Reidy et al., 2013; Kasana et al., 2016; Kabir et al., 2018). Many studies investigated the toxicity of AgNPs and CuONPs on different organisms and human cells and have found their impact 
to be harmful to public health (Colvin, 2003; Handy et al., 2008; Reidy et al., 2013; Kabir et al., 2018).

One route nanoparticles enter the environment is by their release into wastewater and subsequently, sludge and biosolids. During the wastewater treatment process, a large portion of the nanoparticles are deposited into the sludge (Kiser et al., 2010; Kaegi et al., 2011; Reidy et al., 2013). Wastewater sludge is typically treated and stabilized to reduce its human pathogen load; anaerobic digestion is one major sludge stabilization methods used where different groups of microorganisms cooperate to convert a significant portion of the sludge into biogas and reduce the pathogenic content of the remaining sludge. Treated sludge is called biosolids, which frequently used on land as fertilizer.

Using anaerobically digested sludge as a soil fertilizer is widely practiced due to the many benefits it offers for the soil and plants, including, improvement in soil nutrient content, increased water holding capacity, and improvement in soil structure and texture (CCME, 2012; Larney \& Angers, 2012). Thus, biosolids land application is a practical method for sludge disposal, and biosolids are an inexpensive soil fertilizer. However, applying sludge on soil can cause environmental problems, including uploading the soil with toxic compounds and metals, including nanoparticles (Blaser et al., 2008; Gottschalk et al., 2009; Sun et al., 2016; Pandiarajan et al., 2016; Kasana et al., 2016). Due to concerns about soil contamination with AgNPs and CuONPs, several studies have been conducted to assess the toxic impact on soil biological activities (Kumar et al., 2011; Shoults-Wilson et al., 2011; Khurana et al., 2014; Schlich et al., 2016). Studies have revealed that even very low concentrations of AgNPs and CuONPs, which are typically used as antibacterial agents, 
can affect soil microbial activities (Kumar et al., 2011; He et al., 2016; Grün et al., 2018).

This is significant, due to the importance of soil organisms in organic matter decomposition and nutrient cycling.

Soil is the basis for a wide variety of organisms that collaborate and work together to maintain terrestrial ecosystems. Soil microbial communities are highly diverse and represent the fundament of soil functional processes as they play an essential role in biogeochemical processes in soil, including decomposition of organic matter and nutrient cycling (Kennedy \& Smith, 1995; Gattinger et al., 2008; Delgado-Baquerizo et al., 2016; Asadishad et al., 2018). The health of soil microbial communities is essential and critical, since many environmental functions directly depend on it, including decomposition of contaminants, protection against pathogens, purity of groundwater and plant growth development (Kibblewhite et al., 2007; Gattinger et al., 2008). Changes in microbial diversity and population could potentially affect ecosystem quality and health (Kennedy \& Smith, 1995; Alkorta et al., 2003). Soil microbial activities are also sensitive to several environmental changes, including $\mathrm{pH}$, moisture content, temperature, agricultural management and practices and metal contamination (Wang et al., 2017).

Regardless of the method used to apply nanoparticles (directly or through biosolids), reports demonstrate that AgNPs and CuONPs can be anti-microbial and can affect the diversity structure and species composition of soil bacteria including beneficial soil bacteria (Gajjar et al., 2009; Kumar et al., 2011; Colman et al., 2013; Frenk et al., 2013; Kumar et al., 2014; Xu et al., 2015; Samarajeewa et al., 2017; Grün et al., 2018). For example, He et al. (2016) reported that AgNPs in soil at concentrations of $0.1,1$ and 10 
$\mathrm{mg} / \mathrm{kg}$ decreased soil microbial metabolic activity, nitrification and the abundance of ammonia-oxidizing bacteria. Also, Samarajeewa et al. (2017) showed that AgNPs concentrations of $49,124,287,723$, and $1,815 \mathrm{mg} / \mathrm{kg}$ in soil affected microbial composition and functions. CuONPs at concentrations of 0.1 and $1 \mathrm{mg} / \mathrm{kg}$ soil significantly lowered bacterial enzyme activity and changed bacterial community composition (Frenk et al., 2013). In addition, Xu et al. (2015) reported that CuONPs at concentrations of 100, 500 , and $1000 \mathrm{mg} / \mathrm{kg}$ in soil significantly decreased soil microbial biomass carbon, total phospholipid fatty acids and enzyme activity. Therefore, studying the impact of AgNPs and $\mathrm{CuONPs}$ on soil microbial communities is very important. A number of studies have been published regarding the application of AgNPs and/or CuONPs on soil through biosolids, including Colman et al. (2013); McGee et al. (2017); Liu et al. (2017); and Asadishad et al. (2018). However, there is minimal information on their impact when present in biosolids which have had different sludge treatments before reaching the soil. Assessing the impact of nanoparticles directly applied to soil could miss information regarding the modification and behavioral changes they undergo before being applied. Thus, a more realistic approach would be to assess soil exposed to biosolids containing nanoparticles that have been through the wastewater treatment process.

Accordingly, this study investigated the impact of AgNPs and CuONPs individually, on soil biological activities after applying biosolids that have been anaerobically digested. The soil biological activity was studied by comprehensive examination and analysis of the bacterial populations comparing the diversity of the control and four soil microcosms containing two different concentrations of AgNPs and CuONPs. The objectives of this study were to examine changes in the bacterial composition of the soil, identify the 
dominant species and compare the reactors to better understand the impact of the AgNPs and $\mathrm{CuONPs}$. The identification and assessment of the bacteria potentially involved in organic matter decomposition and nutrient recycling was successfully achieved by conducting a microcosm experiment under controlled environmental conditions, with chemical and biological assessments including that for metagenomic analysis. Metagenomic data was generated by high-throughput sequencing of the 16S rRNA gene, using the MinION to explore bacterial populations and diversity. Bacterial composition was studied and analyzed by comparing the soil microcosms with nanoparticles to the control and each other.

\subsection{Materials and methods}

\subsubsection{Silver and copper oxide nanoparticles}

The stock suspensions in water of AgNPs ( $99.9 \%$ pure) and CuONPs (99.95\% pure) were purchased from nanoComposix Inc. (San Diego, CA, USA) and US Research Nanomaterials Inc. (Houston, USA), respectively and maintained at $4 \pm 0.2^{\circ} \mathrm{C}$. Both nanoparticles suspensions are stable and insoluble in milli-Q water (nanopore water). The AgNPs and CuONPs characteristics are listed in Table 5-1. 
Table 5-1 AgNPs and CuONPs characteristics.

\begin{tabular}{|l|l|l|}
\hline Characteristics & AgNPs & CuONPs \\
\hline Diameter (TEM) & $23.1 \pm 6.9 \mathrm{~nm}$ & $25-55 \mathrm{~nm}$ \\
\hline Surface area (TEM) & $21.5 \mathrm{~m}^{2} / \mathrm{g}$ & $29 \mathrm{~m}^{2} / \mathrm{g}$ \\
\hline Particle concentration & $7.2 \times 10^{13}$ particles $/ \mathrm{mL}$ & $9 \times 10^{13}$ particles $/ \mathrm{mL}$ \\
\hline $\mathrm{pH}$ of the solution & 6.3 & $7-9$ \\
\hline Particle surface coating & $\begin{array}{l}\text { polyvinylpyrrolidone } \\
\text { (PVP) }\end{array}$ & Bare \\
\hline Solvent & Milli-Q water & Milli-Q water \\
\hline Morphology & Nearly spherical & Nearly spherical \\
\hline
\end{tabular}

\subsubsection{Soil and sludge characteristics and preparation}

Topsoil from Artistic Landscape Designs Ltd., Ottawa, Ontario, Canada, was used without compost or chemical fertilizer. The soil is characterized as having a loamy texture with a $6.8 \mathrm{pH}$ and $30.78 \mathrm{meq} / 100 \mathrm{~g}$ cation exchange capacity (CEC) and contained $5840 \mathrm{mg} / \mathrm{kg}$ total organic carbon (TOC), $740 \mathrm{mg} / \mathrm{kg}$ total nitrogen $(\mathrm{TN})$ and $15 \mathrm{mg} / \mathrm{kg}$ total phosphorus (TP). Soil samples were sifted through a $2 \mathrm{~mm}$ open sieve to remove any visible roots and rocks, then air dried and stored in clean sealed bags at $4^{\circ} \mathrm{C}$ until use. Three different types of sludge; primary sludge, thickened waste activated sludge and anaerobically digested sludge, were collected from the Robert O. Pickard Environmental Center (ROPEC) wastewater treatment plant in Ottawa (ON, Canada) and used for the sludge anaerobic digestion. The characteristics of the sludge are shown in Table 5-2. 
Table 5-2 Characterization of the three types of raw wastewater sludge.

\begin{tabular}{|l|l|l|l|}
\hline \multirow{2}{*}{ Parameters } & \multicolumn{2}{|l|}{ Substrate } & $\begin{array}{l}\text { Anaerobically } \\
\text { digested sludge } \\
\text { (Inoculum) }\end{array}$ \\
\cline { 2 - 4 } & Primary sludge & tWAS & $7.3 \pm 0.02$ \\
\hline $\mathrm{pH}$ & $7.6 \pm 0.1$ & $7.0 \pm 0.1$ & $19.0 \pm 0.2$ \\
\hline Total solids $(\mathrm{TS})(\mathrm{g} / \mathrm{kg})$ & $42.7 \pm 7.2$ & $52.7 \pm 1.6$ & $15.6 \pm 0.1$ \\
\hline Volatile solids (VS) $(\mathrm{g} / \mathrm{kg})$ & $28.7 \pm 3.5$ & $38.6 \pm 1.5$ & $19.8 \pm 1.1$ \\
\hline $\begin{array}{l}\text { Total chemical oxygen demand (tCOD) } \\
\text { (g/kg) }\end{array}$ & $52.0 \pm 8.3$ & $73.9 \pm 2.1$ & $8.4 \pm 0.2$ \\
\hline $\begin{array}{l}\text { Soluble chemical oxygen demand } \\
\text { (sCOD) }(\mathrm{g} / \mathrm{kg})\end{array}$ & $2.0 \pm 1.1$ & $8.2 \pm 0.1$ & \\
\hline
\end{tabular}

Data are the mean of three replicate measurements \pm standard deviation.

\subsubsection{Experimental design}

The experiment design involved two stages; the first stage involved creating anaerobically digested sludge using mesophilic biochemical methane potential (BMP) assays. The BMP assays were carried out at a mesophilic temperature $\left(34 \pm 1^{\circ} \mathrm{C}\right)$ using an equal amount of primary sludge and thickened waste activated sludge (tWAS) as the substrate and anaerobically digested sludge as the inoculum. The BMP tests were performed using 125 mL Erlenmeyer flasks sealed with rubber stoppers. Each Erlenmeyer flask was filled with a volume of $85 \mathrm{~mL}$ sludge (a mixture of $70 \mathrm{~mL}$ substrate and $15 \mathrm{~mL}$ inoculum), equal amounts of $\mathrm{NaHCO}_{3}$ and $\mathrm{KHCO}_{3}$ were added to achieve alkalinity of $4000 \mathrm{mg} / \mathrm{L}$ (as $\mathrm{CaCO}_{3}$ ), then the nanoparticles were added. There were five BMP reactors; the control (no nanoparticles added), reactor A with $2 \mathrm{mg} \mathrm{AgNPs/g} \mathrm{TS}$; reactor B with $30 \mathrm{mg} \mathrm{AgNPs} / \mathrm{g}$ TS; reactor C with $2 \mathrm{mg}$ CuONPs/g TS and reactor D with $30 \mathrm{mg}$ CuONPs/g TS sludge. The lower nanoparticle concentration represents the higher range of that predicted in the literature and environmental occurrence concentrations in the wastewater sludge (CCME, 2010; EPA, 2009; Gottschalk et al., 2013); the high concentration was chosen to be able to observe the potential impact of accumulated nanoparticles, as well as based on previous 
studies (Wang \& Chen, 2016). The reactors were kept in darkness on the shaker at 100 $\mathrm{rpm}$ in a temperature-controlled incubator for 40 days. Several periodic analyses were done, including biogas collection, TS, VS, and COD (both total and soluble) measurements. During the reactors' preparation and the periodic analyses, nitrogen gas sparging was applied to prevent exposure to air. In addition, to achieve a substrate to inoculum ratio (S/I) of 1.5, the anaerobically digested sludge was centrifuged at $1800 \mathrm{rpm}$ for $10 \mathrm{~min}$ and the pellets were used as inoculum.

After the anaerobic digestion process, the produced sludge was applied on soil to examine the impact of AgNPs and CuONPs on soil biological activities. The biosolids land application experiment was conducted using soil microcosms that were made using clean, sterilize $100 \mathrm{~mL}$ glass beakers and each contained $80 \mathrm{~g}$ of soil. There were five unique microcosms: the control received anaerobically digested sludge without nanoparticles, reactor A received anaerobically digested sludge with $2 \mathrm{mg}$ AgNPs/g sludge; reactor B received anaerobically digested sludge with $30 \mathrm{mg} \mathrm{AgNPs/g} \mathrm{sludge;} \mathrm{reactor} \mathrm{C}$ received anaerobically digested sludge with $2 \mathrm{mg}$ CuONPs/g sludge; and reactor D received anaerobically digested sludge with $30 \mathrm{mg}$ CuONPs/g sludge. The sludge was mixed with a magnetic stirrer for $15 \mathrm{~min}$ then applied to the soil in liquid form; to ensure thorough mixing, the soil was mixed using a spatula. At the beginning of the experiment, the final soil moisture content was adjusted to $60 \%$. The sludge/soil ratio of $0.01 \mathrm{~g} / \mathrm{g}$ (dry weight) was selected based on the biosolids land application guidelines at a rate of 8 ton/ha every 5 years (OME \& OMAFR, 1996). The sludge application ratios used in other studies were also considered (Johansson et al., 1999; Colman et al., 2013; Whitley et al., 2013). The volume of sludge applied was to satisfy two conditions: $1 \%$ sludge/soil ratio and 2 or 30 
mg AgNPs or CuONPs/g sludge. Thus, the final nanoparticles/soil concentration were 20 mg AgNPs/kg soil, $300 \mathrm{mg}$ AgNPs/kg soil, $20 \mathrm{mg} \mathrm{CuONPs/kg}$ soil, and $300 \mathrm{mg}$ $\mathrm{CuONPs} / \mathrm{kg}$ soil CuONPs for reactor $\mathrm{A}, \mathrm{B}, \mathrm{C}$ and $\mathrm{D}$, respectively, which also corresponded to previous studies (Blaser et al., 2008; Kumar et al., 2012; Dimkpa et al., 2015; Samarajeewa et al., 2017). The microcosms were incubated at a constant temperature of 22 $\pm 1^{\circ} \mathrm{C}$ (room temperature), with day/night cycles of $8 / 16 \mathrm{~h}$. The microcosms run for 105 days, and to verify the soil activity, chemical and biological analyses were conducted every 15 days. A 45\% moisture content was maintained in all soil microcosms, which was monitored every three days and adjusted by the addition of sterile distilled water.

\subsubsection{Physicochemical analyses}

The chemical analyses were performed on the soil and sludge before commencing the experiment then periodic chemical analyses were done for each microcosm at seven time points: $0,15,30,45,60,75$ and 105 days. Three replicates were done for each analysis and their means and standard deviations are reported. The soil was analyzed for TOC, TN and TP using $0.01 \mathrm{M}$ calcium chloride as an extraction reagent (Houba et al., 2000). TOC was measured using TOC-VCPH/CPN from Shimadzu and analyzed using TOC-control V software, and CEC was calculated according to procedures reported by (Rebecca, 2004). COD, TN and TP were determined for sludge with HACH methods 8000, 10072 and 10127, respectively (HACH Canada). TS and VS were determined for sludge based on the Part 2540 Standard Methods (APHA et al., 2017). pH levels were measured directly for sludge, and for soil by suspending $5 \mathrm{~g}$ of soil in $5 \mathrm{~mL}$ of water $(1 \mathrm{~g}: 1 \mathrm{~mL}$ ratio), vortexing the suspension for $30 \mathrm{~s}$, then taking the $\mathrm{pH}$ reading using the Orion-thermo scientific device 
(Orion Star TM A326 pH/DO portable meter). Moisture content (MC) was determined by drying $5 \mathrm{~g}$ of the soil sample in the oven at $105^{\circ} \mathrm{C}$ for $24 \mathrm{~h}$. The difference in the sample weight before and after drying divided by the weight of the dried sample and multiplying by $100 \%$ was the MC.

\subsubsection{Biological analyses}

Biological analyses were also carried out at seven time points: 0, 15, 30, 45, 60, 75 and 105 days for each soil reactor.

\subsubsection{Live/Dead bacterial viability staining assay}

The distinction between viable and non-viable cells in the microbial population was assessed by staining with fluorescent dyes according to manufacturer instructions in the BacLight Live/Dead viability assay kit L13152 literature (Molecular Probes, Eugene OR). The soil samples were prepared for staining by suspending $0.1 \mathrm{~g}$ of soil in $0.9 \mathrm{~mL}$ of phosphate buffered saline (PBS) solution with $0.1 \%$ Tween 80 , vortexing for $30 \mathrm{~s}$ at high speed, then allowing the suspension to settle for $30 \mathrm{~min} .20 \mu \mathrm{L}$ of the supernatant was aliquoted into a $1.5 \mathrm{~mL}$ sterilize plastic tube, mixed with an equal volume of the staining reagent and incubated in darkness for $15 \mathrm{~min} .17 \mu \mathrm{L}$ of the mixture was placed between a slide and a $22 \mathrm{~mm}$ square coverslip. The samples were then evaluated using a Nikon Eclipse Ti (Nikon, Montreal) inverted fluorescence microscope. Three tests were done for each soil sample, and three random fields of views (FOV) were chosen on the slide where images were taken. Three images were taken at each FOV using 3 UV filters, GFP-1, Cy-3 and GFPHQ to capture live, dead, and a combination of live and dead microbial cells 
respectively. The three sets of images were assessed, and the most representative was chosen to report. The images were captured with the Q Imaging Retina Exi Fast 1394 camera (Nikon, Montreal QC) and the image analysis was performed with NIS-Elements software (Nikon, Montreal QC).

\subsubsection{Heterotrophic plate counts (HPC)}

Tryptic soy agar (TSA) was used as the growth medium for HPC. TSA Petri plates were prepared first, by adding $30 \mathrm{~g}$ of TSA powdered medium to $1 \mathrm{~L}$ distilled water, dissolved by boiling and autoclave sterilized, After the TSA had cooled to approximately $50^{\circ} \mathrm{C}$ a volume of $22 \mathrm{~mL}$ was poured into each sterile Petri dish $(150 \mathrm{~mm})$ and stored at $3^{\circ} \mathrm{C}$ for a maximum of one week. Soil samples were prepared by suspending $0.1 \mathrm{~g}$ of soil in $0.9 \mathrm{~mL}$ PBS (with $0.1 \%$ Tween 80 ), vortexed for $30 \mathrm{~s}$, then used to make serial dilutions. Three replications of $0.1 \mathrm{~mL} 10 \mathrm{E}+04,10 \mathrm{E}+05$ and $10 \mathrm{E}+06$ dilutions were inoculated onto the TSA plates and incubated in darkness at $34 \pm 1{ }^{\circ} \mathrm{C}$. Colony forming units (CFU) were calculated after $24 \mathrm{~h}$ of incubation. HPC was conducted using the spread plate method, according to Standard Methods, Part 9215 C (APHA et al., 2017).

\subsubsection{DNA extraction, quantification and quality control}

Genomic DNA was extracted from the soil using the PowerSoil DNA Isolation Kit from MO BIO Laboratories Inc. (QIAGEN Online Shop). Before extraction, the soil samples were prewashed to reduce inhibitors affecting DNA extraction and downstream qPCR. The prewashing was performed according to the method described by Kibbee and Ormeci (2017), with the exception that the samples were soil. $1 \mathrm{~g}$ soil was mixed with $1 \mathrm{~mL}$ PBS, 
vortexed for $15 \mathrm{~s}$ at high speed and centrifuged at $8000 \times g$ for $5 \mathrm{~min}$. The supernatant was then discarded, and the pellet used for DNA extraction. Soil genomic DNA was extracted for each sample in triplicate following the manufacturers' instruction, with the exception of the cell lysis and inhibition removal steps, for which the incubation times were doubled from five to ten minutes. The DNA concentration was measured using the Qubit dsDNA HS Assay Kits with the Qubit 3.0 Fluorometer from Molecular Probes (Life Technology, Eugene, OR). The purity of the DNA was assessed by measuring the absorbance ratios at 260/280 and 260/230 nm, and the congruence of concentrations with Qubit values using the NanoDrop 1000 Spectrophotometer (Thermo Scientific, Wilmington, USA). The genomic DNA was also assessed using gel electrophoresis to ensure fragment sizes were between 3 and $10 \mathrm{Kbps}$, as required for optimal MinION sequencing. Genomic DNA extracts were run on a $0.8 \%$ agarose gel at $7 \mathrm{~V} / \mathrm{cm}^{2}$; a TAE buffer was used to prepare the agarose gel. 6X gel loading dye and GeneRuler $1 \mathrm{~Kb}$ ready-to-use DNA Ladder from Thermo Fisher were used to visualize the genomic DNA size. The DNA samples were stored at $-20^{\circ} \mathrm{C}$ until use.

\subsubsection{Bacterial composition analysis}

The bacterial composition structure profiles of the soil samples were analyzed using the 16S rRNA BLAST results generated by the MinION sequencing runs and EPI2ME workflows. 


\subsection{High-throughput sequencing}

Five genomic DNA libraries were prepared following the 16S Barcoding Kit (SQKRAB204) protocol and used to generate the $16 \mathrm{~S}$ bacterial community profiles. Each library, consisting of seven uniquely barcoded time points, was run separately in a MIN 107 MinION $^{\mathrm{TM}}$ flowcell. The basecalled data from each run was used in the EPI2ME Fastq 16S workflow, which generated BLAST data that was directly used for diversity and community analyses. The MinION ${ }^{\mathrm{TM}}$, flowcell and kits were purchased from Oxford Nanopore Technologies, New York, USA, and the library preparation and sequencing were done according to the MinION 1D 16S Barcoding Kit (SQK-RAB204) protocol. PCR was used to amplify the DNA samples by using specific $16 \mathrm{~S}$ primers (27F and 1492R) with barcodes, and 5' tags that facilitate ligase-free attachment of Rapid Sequencing Adapters. The PCR products were cleaned, concentrated with Agencourt AMPure XP beads and quantified with the Qubit dsDNA HS Assay Kit using the Qubit 3.0 Fluorometer (Molecular Probes, Life Technology, Eugene, OR). The PCR products for each library were then pooled at the required ratio, a rapid sequencing adapter attached, and then introduced and attached to loading beads which were then loaded into the MinION flow cell. The MinION protocol uses software named MinKNOW which allowed for real-time basecall monitoring; each library sequencing run was stopped at $\sim 150,000$ reads to maintain consistent read depths for all soil sample libraries.

\subsubsection{Metagenomic analysis}

The Fastq files generated by MinKNOW were BLASTed against the NCBI 16S bacterial database, using the Oxford Nanopore's cloud-based analysis platform EPI2ME with the 
16S Fastq workflow. Each read was classified according to the percentage of coverage and identity, then the results were transcribed to Excel datasheets and sorted into different taxonomy classifications (mainly phylum and genus levels) for every time point and each soil reactor. A 3\% sequence dissimilarity cut-off value, typically used to estimate the level of phylotype species diversity, was applied universally. The biodiversity was estimated for the highest detected phyla with a relative abundance of $\geq 0.01 \%$. Alpha biodiversity (Shannon-Wiener index, evenness, and richness) and Beta-Euclidean biodiversity were estimated and analyzed for bacteria at the phylum level. The relative abundance was estimated and compared for each microcosm at both phylum and genus levels. The genus level cut off value for biodiversity analysis was $\geq 1 \%$ relative abundance. Several interesting genera were also discussed, including those with a relative abundance of $\leq 1 \%$. These genera were selected based on their important role in soil organic matter decomposition and nutrient recycling, as cited in the literature, and are shown in Table S1. Shannon-Wiener index, used to characterize the phyla diversity in a community, was determined by calculating the percentage of a phylum to the other phyla in a community, then multiplying it by the natural logarithm of this proportion. The resulting values are summed across the phyla and multiplied by -1 (Equation 5-1) (Magurran, 1988). Evenness was calculated according to Equation 5-2 (Magurran, 1988; Mulder et al., 2004). Evenness is between 0 and 1 , with 1 being complete evenness. Richness $(\mathrm{N})$ is the total number of phyla after the cut-off. 
$H=-\sum_{i=1}^{N} p i \ln p i$

$E=\frac{H}{H \max }=H / \ln N$
Equation 5-1

\section{Equation 5-2}

Where $\quad H$ : Shannon-Wiener index

$N$ : total number of phyla

$p i$ : proportion of phylum $i$ to all other phyla

$E$ : phyla evenness

Beta-Euclidean diversity was calculated according to Equation 5-3 (Edgar, 2018).

Beta - Euclidean $=\operatorname{sqrt}\left(\sum_{\mathbf{1}}^{\boldsymbol{N}}(\boldsymbol{n} \boldsymbol{k i}-\boldsymbol{n}\right.$
Where
$\begin{aligned} & N: \text { total number of phyla } \\ & n k i: \text { count of OTU } k \text { in sample } i \\ & n k j: \text { count of OTU } k \text { in sample } j\end{aligned}$

\subsubsection{Statistical analyses}

A one-way analysis of variation (ANOVA) at $95 \%$ confidence level was used to study and evaluate significant differences between the soil reactors; the difference was considered significant if $\mathrm{p}$-values were $<0.05$. Accordingly, if there were significant differences between the reactors, the Post Hoc Tukey test was used to further separate mean values, using the same significance level (Currell \& Dowman, 2005). Three replicates were done for each analysis and the results were reported with the mean \pm standard deviations. To compare values within the same group, the coefficient of variance (CV) was applied to compare individual data set, if the $\mathrm{CV}$ is $\leq 0.05$ then the data is not significantly different (i.e., the confidence is $\geq 95 \%$ ). 


\subsection{Results and discussion}

\subsubsection{Soil physicochemical properties}

Organic matter is an extremely important soil property as it is essential for plant growth and is approximately 56\% organic carbon, the source of energy and nutrients for soil microorganisms (Edwards et al., 1992). Organic matter, such as humus, helps maintain the stability of aggregates and nutrients, and is necessary for the water holding capacity of the soil. Organic carbon compounds (e.g., polysaccharides) help hold mineral particles together as microflocs. Changes in organic matter can affect the functional activity of soil. In this study, the TOC of the soil reactors was examined, and the results showed similar trends over time with no significant difference between the control and soil microcosms with AgNPs or CuONPs, shown in Figure 5-1(a). This indicates that these nanoparticles have a minimal impact on soil TOC content. Comparing the control to the other soil microcosms, the T-test $\mathrm{p}$-values were all $>0.05$, which agrees with the results of previous studies. Ben-Moshe et al. (2013) showed that CuONPs did not cause changes in the soluble organic matter (SOM) and total organic matter (TOC) in the soil extract, though they did find some changes in humic substances. In addition, Carbone et al. (2014) and Hänsch \& Emmerling (2010) found no significant effects from AgNPs on soil physicochemical parameters, including TOC and $\mathrm{pH}$. The $\mathrm{pH}$ was monitored for the soil microcosms over time, as it is one of the main factors that influence microbial activity in the soil. The $\mathrm{pH}$ was constant and showed similar trends over time in all reactors with no significant

differences (T-test, p-value $>0.05$ ) as shown in Figure 5-1(b). Even though $\mathrm{pH}$ is considered one of the key factors affecting the behavior of nanoparticles, including their 
dissolution, mobility and bioavailability, this effect still depends on the $\mathrm{pH}$ level (Oukarroum et al., 2014; Read et al., 2016). For example, Read et al. (2016) indicated that a $\mathrm{pH}$ of 7.2 did not impact the zinc nanoparticles toxicity in soil. Thus, as $\mathrm{pH}$ range is between 6.9 and 7.7 in this study, it was not a factor that altered the AgNPs and CuONPs behavior and toxicity in soil.
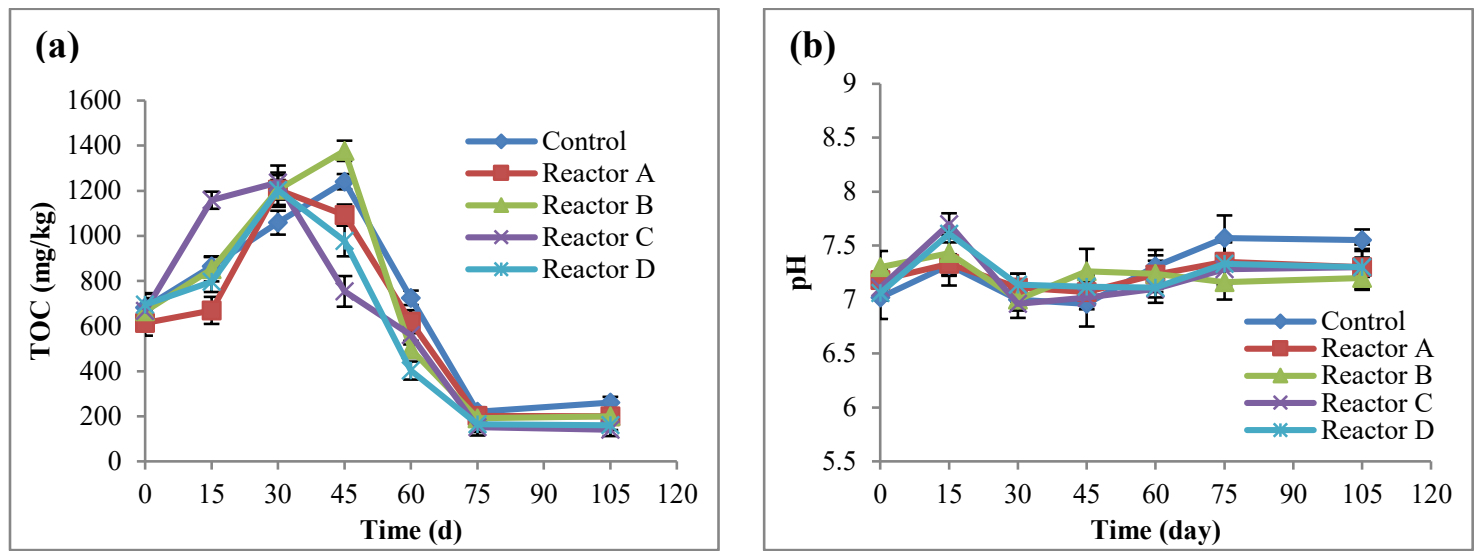

Figure 5-1 Soil concentration of TOC (a) and pH (b) over time (Reactors A, B, C, and D are the soil microcosms, which received anaerobically digested sludge with 2 mg AgNPs/g TS, 30 mg AgNPs/g TS, 2 mg CuONPs/g TS, and $30 \mathrm{mg} \mathrm{CuONPs/g} \mathrm{TS,} \mathrm{respectively).}$

\subsubsection{Impact of AgNPs and CuONPs on the heterotrophic bacteria and percentage of live cells}

Enumeration of heterotrophic bacteria is important because they are a good indicator of soil health. Heterotrophic bacteria refer to the bacteria that use organic carbon to grow. Even though the HPC method generally detects only a small fraction of environmental bacteria (Allen et al., 2004), it does provide a good metric to assess relative changes in numbers of these bacteria within the soil reactors. As shown in Figure 5-2(a), HPC varied significantly between the soil reactors and comparing the control to the other reactors through statistical analysis using T-test showed that all p-values were $<0.05$. Thus, as in 
Figure 5-2, the growth of heterotrophic bacteria was significantly inhibited by the presence of AgNPs and CuONPs, particularly CuONPs, which seemed to be associated with a higher level of inhibition. In addition, the different concentrations of AgNPs and CuONPs had similar effects, as they both induced similar inhibition of heterotrophic bacterial growth (using ANOVA analysis, comparing reactors $\mathrm{A}, \mathrm{B}, \mathrm{C}$, and $\mathrm{D}$, p-value was $>0.05$ ). This indicated that the toxicity of AgNPs and CuONPs on soil heterotrophic bacteria is not dosedependent. The percentage of live cells in the soil microcosms was also investigated over time and the results showed that AgNPs and CuONPs, particularly at the higher concentration, decreased the percentage of live cells compared to the control as shown in Figure 5-2(b). However, the decrease was not significant based on ANOVA analysis (pvalue $>0.05$ ). Representative sample images of Live/Dead staining for each reactor at each time point are shown in Figures S-2 to S-6.
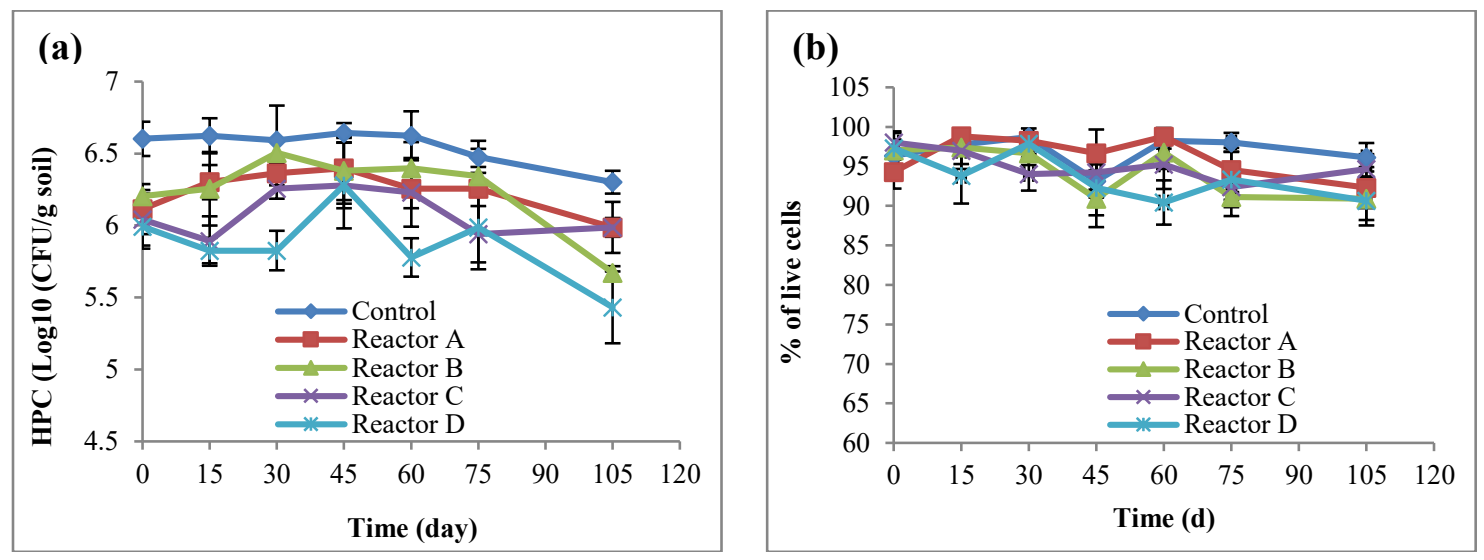

Figure 5-2 Impact of AgNPs and CuONPs on soil microbial growth as measured by the mean \pm standard error of CFU derived from the heterotrophic plate counts (a), and \% of live cells derived from Live/Dead staining assay (b) (Reactors A, B, C, and D are the soil microcosms, which received anaerobically digested sludge with 2 mg AgNPs/g TS, 30 mg AgNPs/g TS, 2 mg CuONPs/g TS, and $30 \mathrm{mg} \mathrm{CuONPs} / \mathrm{g}$ TS, respectively). 


\subsubsection{Impact of AgNPs and CuONPs on bacterial community structure}

DNA (Figure S-1) was analyzed for the bacterial community diversity and phylogenetic structure of all the reactors were analyzed at the phylum and genus levels every 15 days and the sequencing results of the $16 \mathrm{~S}$ rRNA gene showed highly diversified bacterial communities. Table 5-3 shows the relationship between the concentrations of the nanoparticles used, the total number of species or operational taxonomic units (OTUs), the total sequencing reads and the calculated overall Shannon-Wiener index. Bacterial Alpha and Beta-Euclidean diversities were estimated at the phylum level. Alpha diversity is a standard measure of the phyla/genera/species diversity in a community, while BetaEuclidean diversity is a measure used to compare the composition between two communities. Alpha diversity is represented by the Shannon-Wiener index (characterizes the composition and commonness of species in a community), evenness (measures how close in number each species in a community is), and richness (is the number of unique OTUs or phyla/genera/species in a community). As shown in Table 5-3, the overall Shannon-Wiener index and the total number of phyla increased with a higher concentration of AgNPs and CuONPs, while the total number of species (OTUs) decreased. All the soil reactors except reactor A had a higher Shannon-Wiener index than the control. Although the Shannon-Wiener index represents the diversity of a community, it cannot be concluded that the presence of nanoparticles enhanced the bacterial diversity in the soil, as the population of important bacteria in these reactors was low. The control had a higher population of key bacterial phyla such as Proteobacteria (dominant species), which is responsible for most of the soil's beneficial biological activities (Table 5-4) and represents the majority of PGPR than other less important bacteria. This is apparent by the number of 
species represented by unique OTUs for each phylum/ genus in each reactor. Thus, the high concentration of nanoparticles lowered the population of several bacterial phyla/ genus, and the bacterial population was distributed more evenly between the different phyla/genus observed. This was also confirmed by evenness calculations, which found greater evenness in the reactors with nanoparticles as seen in Figure 5-4. Our previous study (Chapter 4) showed similar findings as did other studies, including Huang et al. (2014), who indicated higher doses of tetracycline increased the Shannon-Wiener index, and Hong et al. (2015) who reported an increase in the bacterial diversity indices in heavy metal contaminated soil. In addition, some studies indicated that the antimicrobial properties of nanoparticles can negatively impact the growth of the dominant bacterial species in the sludge by allowing sparse species to thrive, which increases the ShannonWiener index and the number of different phyla presented (Huang et al., 2014; Li et al., 2011; Czárán et al., 2002). The Shannon-Wiener index for the soil reactors was also generated at each time point, as shown in Figure 5-3. The control and reactor A had similar trends, which significantly decreased after $15 \mathrm{~d}$, then increased until day 75 where it dropped again. Reactor B varied from the other reactors, as the Shannon-Wiener index continued to decrease until day 75 . Reactors $\mathrm{C}$ and D both had a significant drop at day 15 , then increased until day 75 where it remained constant. Overall, the Shannon-Wiener index was higher in reactors A, B and C compared to the control and reactor A. Statistical analysis showed that reactors $\mathrm{C}$ and $\mathrm{D}$ were significantly different than the control, based on T-test, p-values were $0.6,0.28,0.04$, and 0.01 for reactors $A, B, C$ and $D$, respectively. 
Table 5-3 MinION sequencing statistics and Shannon-Wiener index for the soil microcosms (all time points combined).

\begin{tabular}{|c|c|c|c|c|c|c|c|}
\hline Reactor & $\begin{array}{c}\text { Nanoparticles } \\
\text { concentration } \\
\text { (mg NPs/g dry } \\
\text { soil) }\end{array}$ & $\begin{array}{c}\text { Overall } \\
\text { number of } \\
\text { raw } \\
\text { sequences }\end{array}$ & $\begin{array}{c}\text { \% } \\
\text { Classified }\end{array}$ & $\begin{array}{c}\text { \% } \\
\text { Average } \\
\text { process } \\
\text { accuracy }\end{array}$ & $\begin{array}{c}\text { Total } \\
\text { number } \\
\text { of phyla }\end{array}$ & $\begin{array}{c}\text { Total } \\
\text { number } \\
\text { of } \\
\text { species } \\
\text { (OTUs) }\end{array}$ & $\begin{array}{c}\text { Shannon- } \\
\text { Wiener } \\
\text { index } \\
\text { (phyla) }\end{array}$ \\
\hline Control & None & 161,223 & 93 & 85 & 24 & 2793 & 0.89 \\
\hline A & 0.02 & 64,840 & 83 & 83 & 20 & 2123 & 0.76 \\
\hline B & 0.3 & 140,699 & 87 & 83 & 25 & 2503 & 1.02 \\
\hline C & 0.02 & 114,458 & 94 & 85 & 21 & 2546 & 1.23 \\
\hline D & 0.3 & 125,443 & 82 & 84 & 28 & 2644 & 1.33 \\
\hline
\end{tabular}

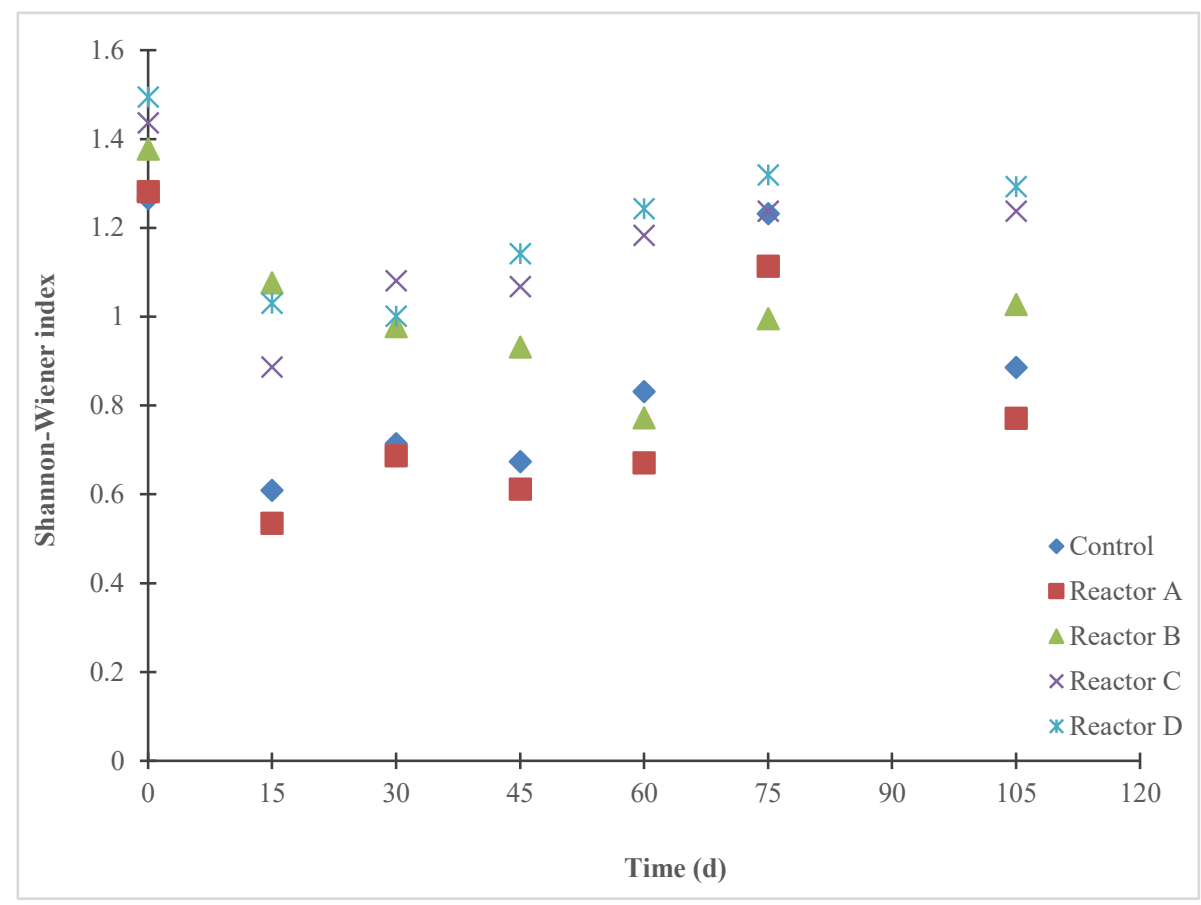

Figure 5-3 Shannon-Wiener index for each soil microcosm over time. Reactors A, B, C, and $D$ are soil microcosms, which received anaerobically digested sludge with 2 mg AgNPs/g TS, $30 \mathrm{mg} \mathrm{AgNPs} / \mathrm{g}$ TS, $2 \mathrm{mg} \mathrm{CuONPs/g} \mathrm{TS,} \mathrm{and} 30 \mathrm{mg} \mathrm{CuONPs} / \mathrm{g}$ TS, respectively.

In addition to the Shannon-Wiener index, phyla evenness and richness were also investigated. There was a total of 17 unique phyla OTUs (relative abundance $\geq 0.01$ ) in each reactor, as depicted in Figure 5-4(b). The phyla evenness was low for the control and 
reactors $\mathrm{A}$ and $\mathrm{B}$ compared to the evenness in reactors $\mathrm{C}$ and $\mathrm{D}$, as indicated in Figure 54(a). The phyla evenness is a measure of biodiversity that quantifies how equal communities are. A higher phyla evenness indicates that the phyla are distributed more evenly in the reactors, as in reactors $\mathrm{C}$ and $\mathrm{D}$; as well, reactor $\mathrm{B}$ also had a slightly higher evenness than the control. This supports what was discussed previously: a higher ShannonWiener index is associated with higher concentrations of nanoparticles.
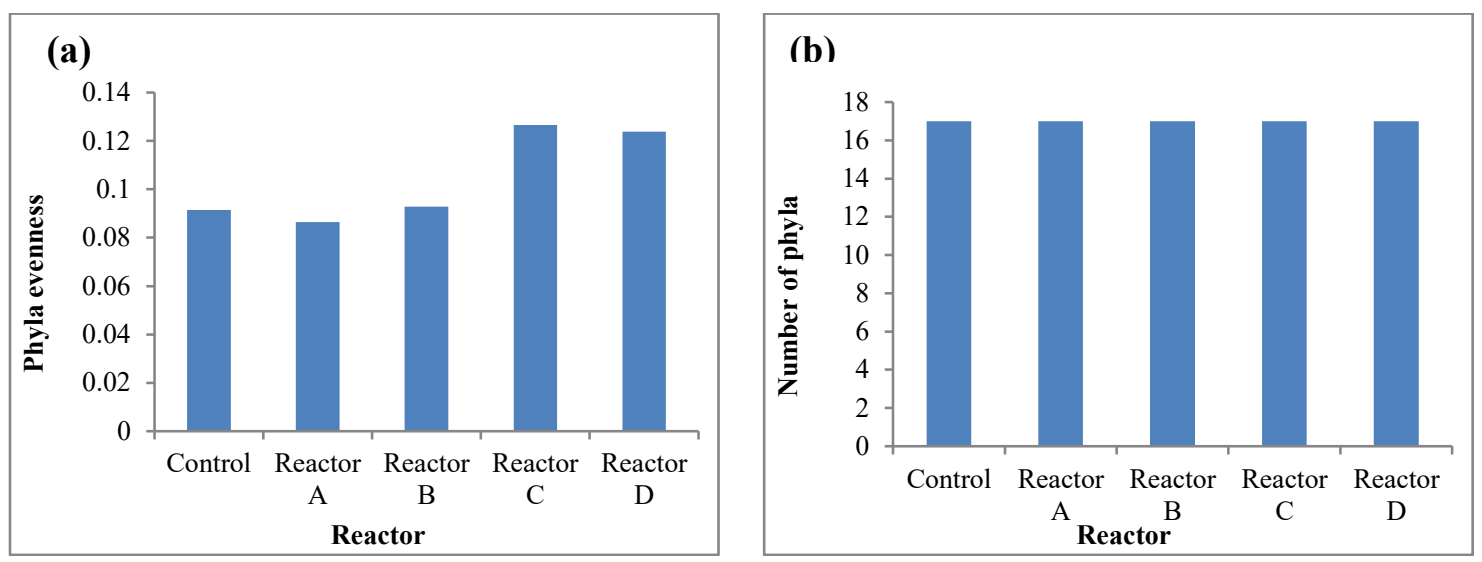

Figure 5-4 Overall phyla evenness (a) and phyla richness, represented by the number of phyla, whose relative abundance $\geq 0.01$ (b) in each reactor. Reactors $A, B, C$, and $D$ are soil microcosms, which received anaerobically digested sludge with $2 \mathrm{mg}$ AgNPs/g TS, $30 \mathrm{mg}$ AgNPs/g TS, 2 mg CuONPs/g TS, and $30 \mathrm{mg} \mathrm{CuONPs} / \mathrm{g}$ TS, respectively.

Beta-Euclidean diversity of bacterial communities in each soil microcosm was also calculated through comparing each soil microcosm to the all other microcosms and hence examine the similarity of each reactor to the others. As shown in Figure 5-5, BetaEuclidean diversity of the control was high compared to the other reactors, which suggests significant differences in taxonomic abundance profiles between the control and other soil reactors. A high Beta-Euclidean diversity indicates relatively low similarity, and a low Beta-Euclidean diversity shows a high level of similarity. This also confirms the high population of dominant species in the control. 


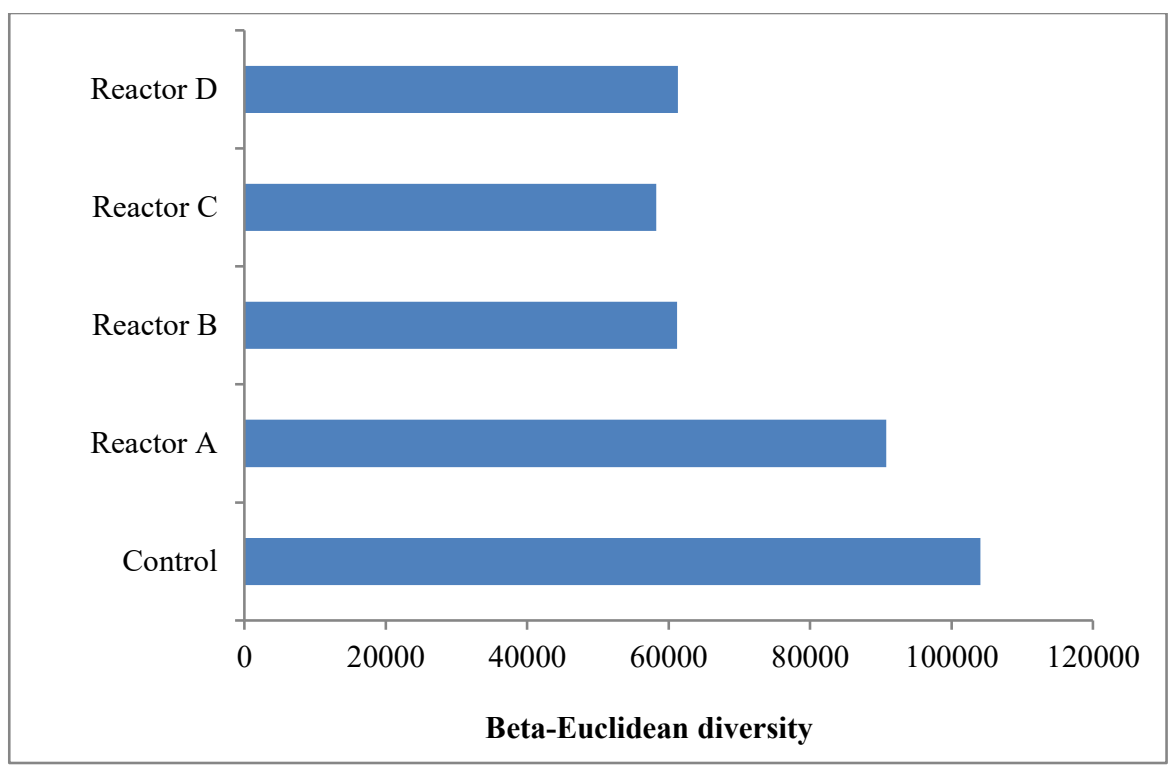

Figure 5-5 Beta-Euclidean diversity, representing the bacterial composition difference between each reactor to all other reactors. Reactors $A, B, C$, and $D$ are soil microcosms, which received anaerobically digested sludge with 2 mg AgNPs/g TS, 30 mg AgNPs/g TS, 2 mg CuONPs/g TS, and $30 \mathrm{mg} \mathrm{CuONPs/g} \mathrm{TS,} \mathrm{respectively.}$

\subsubsection{Abundance and diversity of bacterial phyla}

The classified sequences were associated with 20 to 28 phyla, of which 17 were found to have a relative abundance of $\geq 0.01 \%$ in at least one soil reactor, as shown by the average relative abundances in Figure 5-6. Four major phyla (Proteobacteria Bacteroidetes, Firmicutes and Gemmatimonadetes) were present at relative abundances of more than $1 \%$ and consisted of 91 to $97 \%$ of the total observed phyla OTUs, as seen in Figure 5-6(a). Of these bacterial phyla, Proteobacteria was the most abundant, with 62 to $81 \%$ average relative abundance. The next most abundant group of phyla represented approximately 2 to $9 \%$ of the total observed phyla OTUs, as shown in Figure 5-6(b). The relative abundance of these phyla increased with higher nanoparticle concentrations particularly in soil reactors with CuONPs. This supports what was discussed in Section 5.3.3, where the high concentration of AgNPs and both concentrations of CuONPs caused a higher Shannon- 
Wiener index and an increased number of observed phyla, but also resulted in lower observed unique OTUs at the species level within each phylum. Comparison of the relative abundance of the observed phyla highlighted differences between the control and other reactors, particularly reactors $\mathrm{B}, \mathrm{C}$ and $\mathrm{D}$. Although this appears to suggest that the effect of nanoparticles can alter the abundance of phyla, the differences were not significant, based on statistical analysis using ANOVA that indicated a p-value $>0.05$.
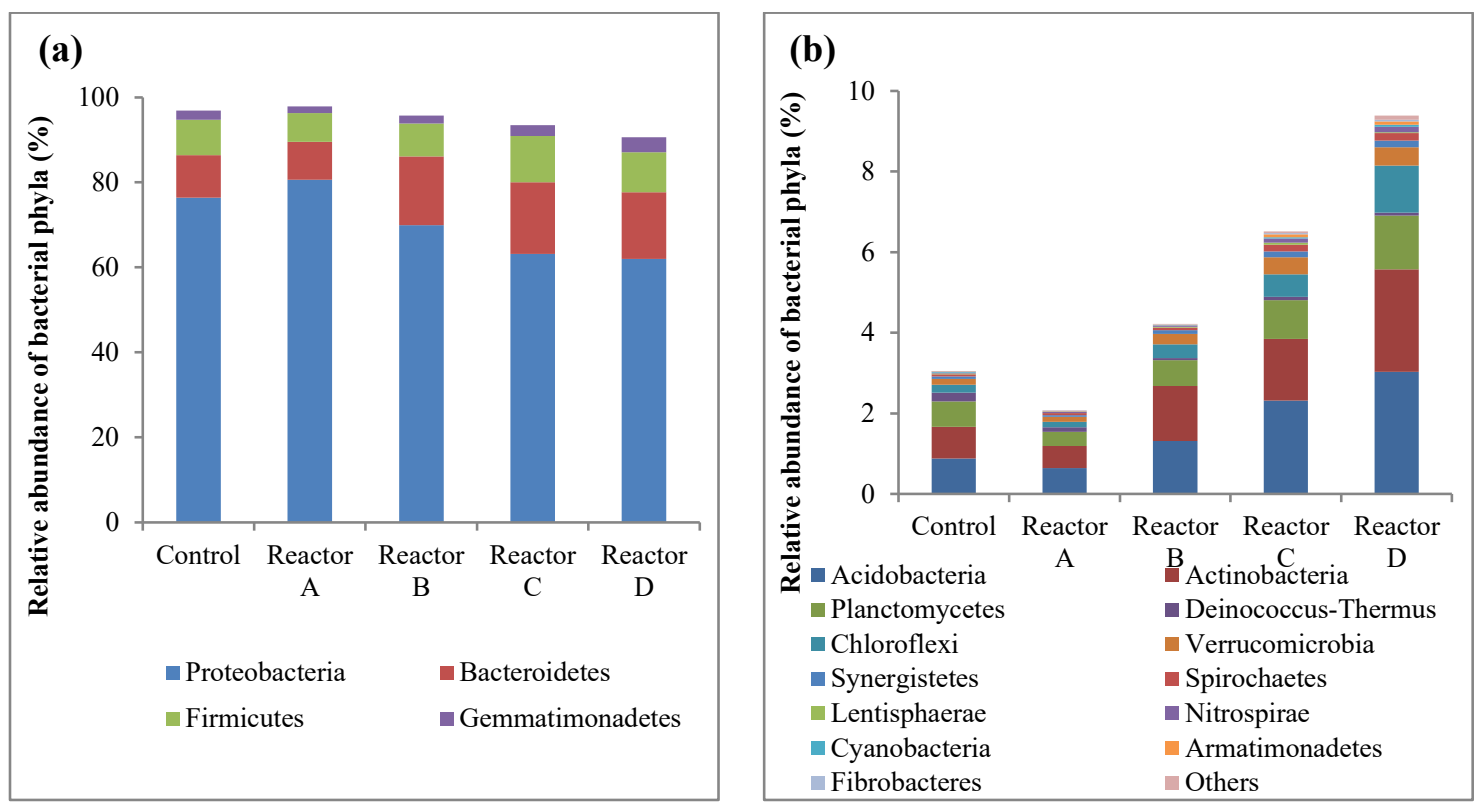

Figure 5-6 Average relative abundance at the phylum level in each soil microcosm. Most abundance phyla $\geq 90 \%$ of the observed OTUs (a), and next most abundant bacterial phyla $\leq 10 \%$ of the observed OTUs (b). Reactors A, B, C, and D are soil microcosms, which received anaerobically digested sludge with $2 \mathrm{mg}$ AgNPs/g TS, $30 \mathrm{mg} \mathrm{AgNPs/g} \mathrm{TS,} 2$ mg CuONPs/g TS, and $30 \mathrm{mg} \mathrm{CuONPs} / \mathrm{g} \mathrm{TS}$, respectively.

Over the duration of the experiment, several phyla were observed, and each showed different OTU trends, as shown in Figure 5-7. Proteobacteria showed different growth patterns in which the control increased until day 45 then it started to drop, while reactors $\mathrm{A}$ and $\mathrm{B}$ increased at similar levels, and reactors $\mathrm{C}$ and $\mathrm{D}$ increased during the first 15 days 
then began to drop. However, ANOVA statistical analysis showed no significant differences, p-value was 0.1. Bacteroidetes also displayed variant trends, as control remained nearly unchanged while reactors A and B were increasing, and reactors C and D were decreasing but there were no significant variations between all the reactors. Firmicutes decreased throughout the experiment in all soil reactors with minimal variation among the reactors, and a p-value of 0.6 . Gemmatimonadetes showed an increase over time in all soil reactors. Reactor D had the highest increase, and all the reactors showed similar trends with no significant differences based on ANOVA analysis which showed p-value of 0.4. Acidobacteria and Actinobacteria increased over time, with only reactor D was significantly different than the control for both phyla. Based on T-test, p-values for reactor D compared to the control were 0.03 and 0.0003 for Acidobacteria and Actinobacteria, respectively. There were also significant variations of Chloroflexi between the control and reactor D at the start of the experiment (p-value was 0.01). However, the Chloroflexi decreased until it reached similar levels to those of the control at day 45 and 105 for reactors C and D, respectively. Generally, at the start of the experiment $(0 \mathrm{~d})$, most of the studied phyla behaved differently due to the AgNPs and CuONPs affecting their abundance, as seen in Figure 5-7. In most cases, AgNPs caused an instant decrease (at day 0), while CuONPs increased the phyla OTUs, but eventually the control and other reactors aligned at a similar level. This can suggest several things, including the presence of high concentrations of silver and copper ions at the start, which eventually decreased due to interaction with soil organic matter, minerals or cations in the soil. For example, Grün et al. (2018) found that most initial AgNPs were attached to the clay particles after an incubation time of two weeks, as seen in TEM images. Another explanation is that over 
time many bacteria can protect themselves from their surroundings. They achieve this through the production of extracellular proteins that can coat AgNPs and CuONPs and reduce their toxicity, as can polysaccharides of soil microbiome (Wu et al., 2010; Sudheer Khan et al., 2011).
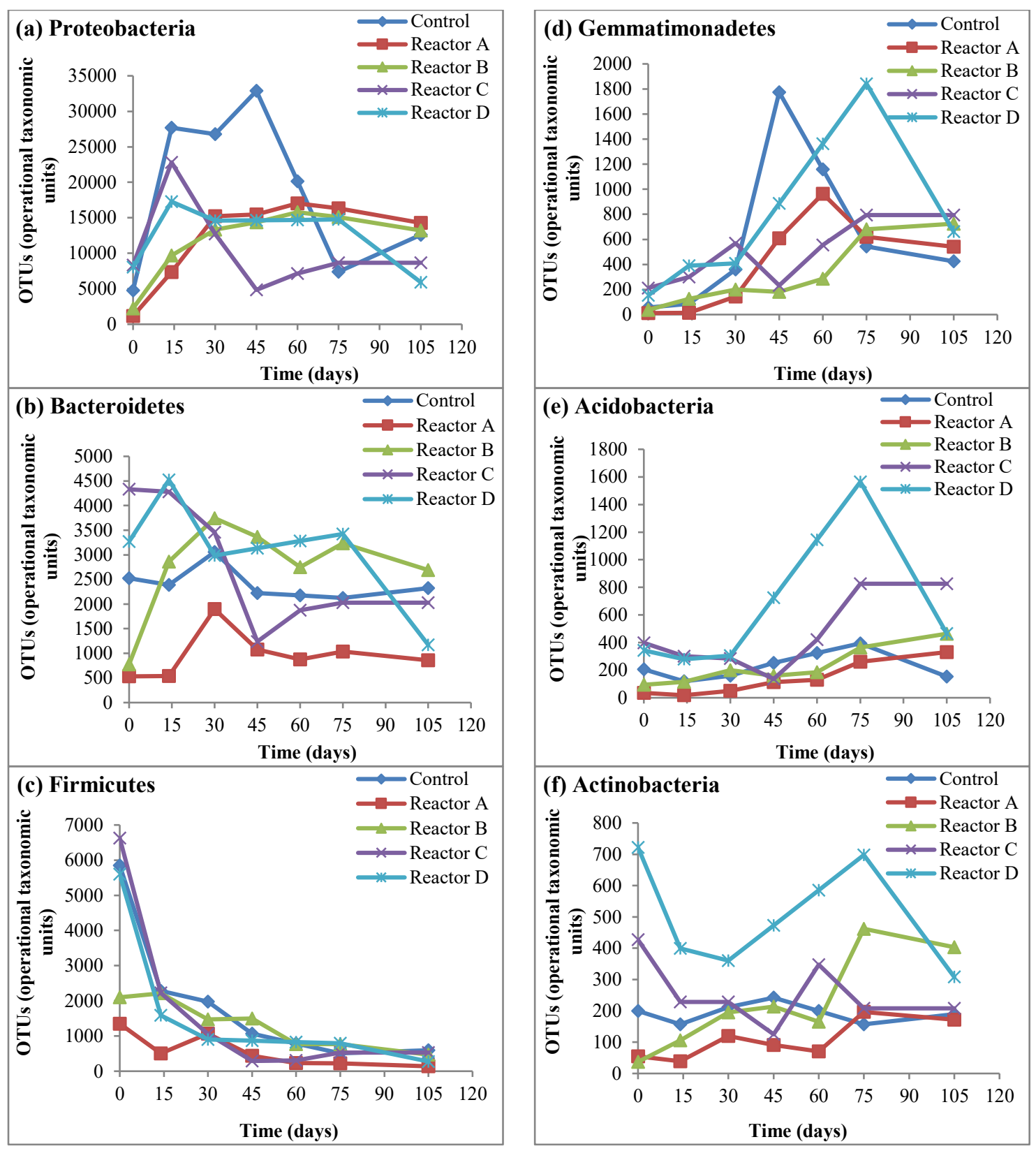

Figure 5-7 OTUs of phyla over time. Reactors A, B, C, and D are soil microcosms, which received anaerobically digested sludge with 2 mg AgNPs/g TS, 30 mg AgNPs/g TS, 2 mg $\mathrm{CuONPs} / \mathrm{g} \mathrm{TS}$, and $30 \mathrm{mg}$ CuONPs /g TS, respectively. 


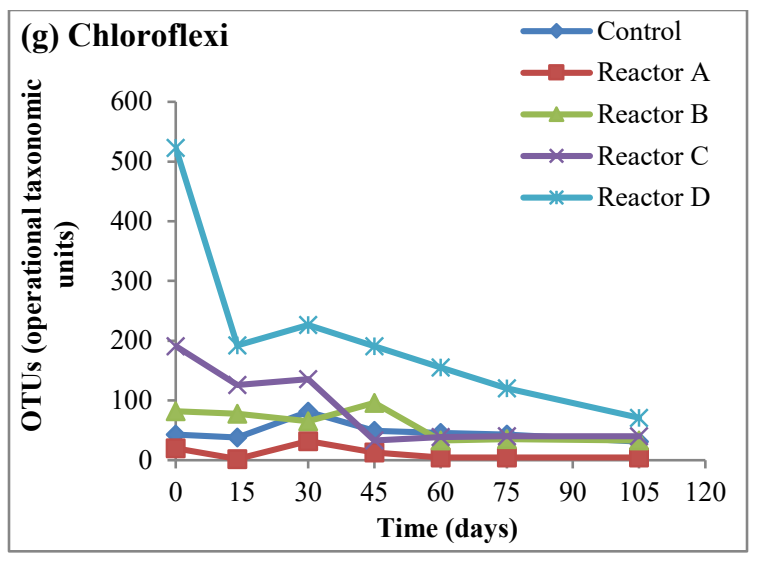

Figure 5-7 (Continued)

\subsubsection{Abundance and diversity of bacterial genera}

The total unique genus OTUs were 562, 472, 505, 509 and 544 in the control and reactors A, B, C and D, respectively indicating that, compared to the control, AgNPs and CuONPs decreased the overall number of observed genera. The top 79 observed genera were studied of which the first group (top 18 genera) were most abundant, as each genus had a relative abundance of $\geq 1 \%$. The second group (61 genera) were the least abundant as each genus had a relative abundance between 0.1 and $1 \%$. As shown in Figure 5-8, there were significant differences in the bacterial genus communities of the control and the other reactors, particularly in reactors $\mathrm{B}, \mathrm{C}$ and $\mathrm{D}$. This indicated that the high concentration of AgNPs (reactor B) and both concentrations of CuONPs caused shifts in bacterial genus abundance. The shifts were more significant for the least abundant genera (61 genera) and the statistical analysis using T-test showed p-values of $0.7,0.007,0.003$, and 0.002 for reactors $\mathrm{A}, \mathrm{B}, \mathrm{C}$ and $\mathrm{D}$, respectively, compared to the control. 


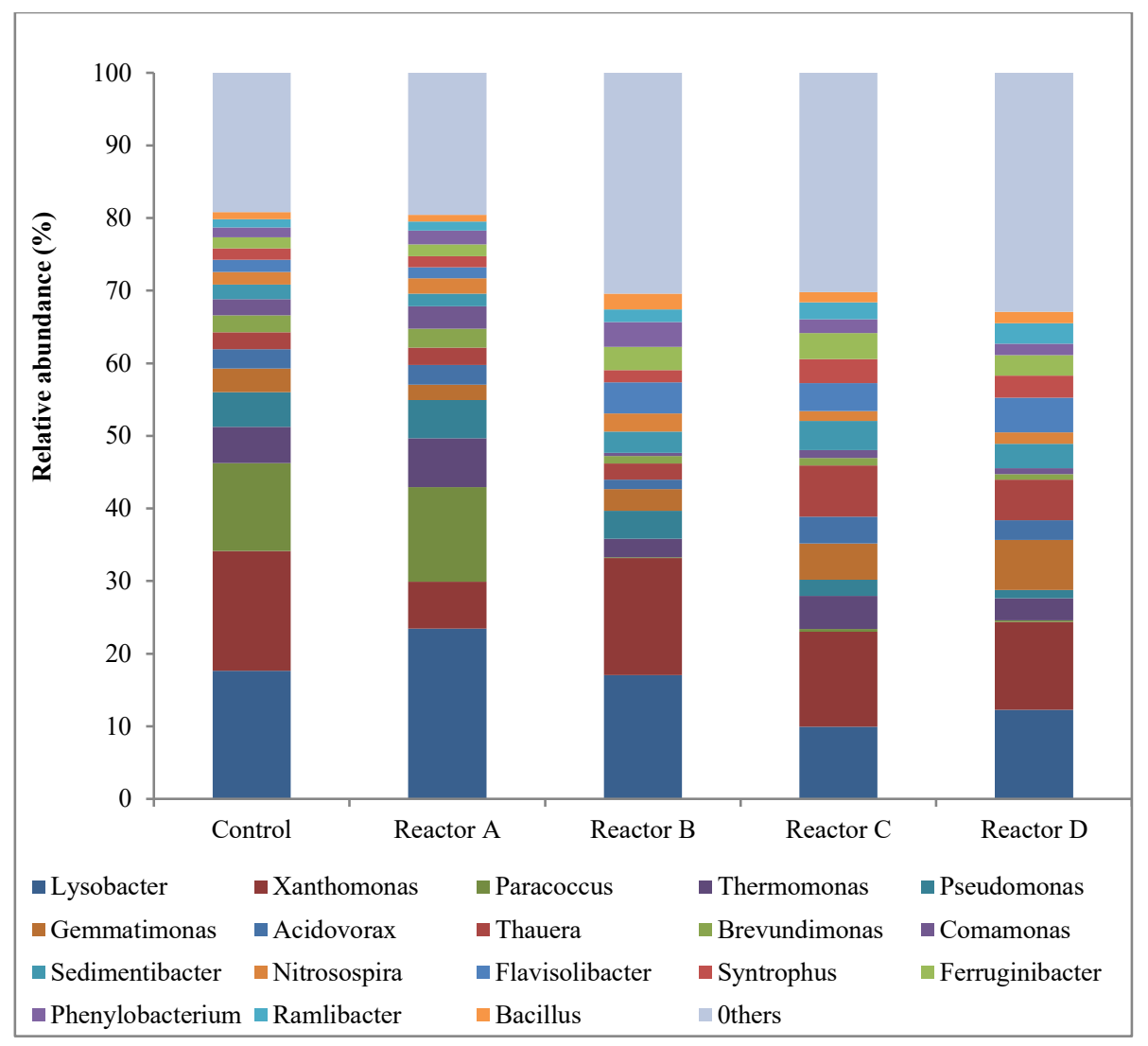

Figure 5-8 The relative abundance of the top 18 genera (each genus with $\geq 1 \%$ relative in all soil reactors), and others representing 61 genera with a relative abundance of $0.1-1 \%$. Reactors A, B, C, and D are soil microcosms, which received anaerobically digested sludge with 2 mg AgNPs/g TS, 30 mg AgNPs/g TS, 2 mg CuONPs/g TS, and 30 mg CuONPs /g TS, respectively.

Thus, the presence of AgNPs and CuONPs affected the detection and relative abundance of many genera including some highly abundant genera, such as Lysobacter, Paracoccus, Thermomonas, Pseudomonas, Brevundimonas, and Comamonas, as shown in Figure 59(a). The most impacted genus was Paracoccus, whose average relative abundance decreased from $12 \%$ in the control to $0.12,0.34$ and $0.22 \%$ in reactors $\mathrm{B}, \mathrm{C}$ and $\mathrm{D}$, respectively. AgNPs and CuONPs also affected several low abundant genera, including Nitrosomonas, Stenotrophomonas, Tissierella, Planomicrobium, and Sphingobium as shown in Figure 5-9(b). In addition, the relative abundance of some highly abundant genera 
increased due to the presence of AgNPs and CuONPs, including Sedimentibacter, Flavisolibacter, Syntrophus, Ferruginibacter and Bacillus, as well as some low abundant genera, as shown in Figure 5-10. The population of genera impacted by AgNPs and CuONPs, $46 \%$ of the OTUs in the control is greater than the population of genera whose population was enhanced by AgNPs and CuONPs, $15 \%$ of the OTUs in control, as seen in Figures 5-9 and 5-10. Many other genera (approximately $39 \%$ of the OTUs in the control) did not show changes in numbers of OTUs in soil reactors with AgNPs than those with CuONPs.
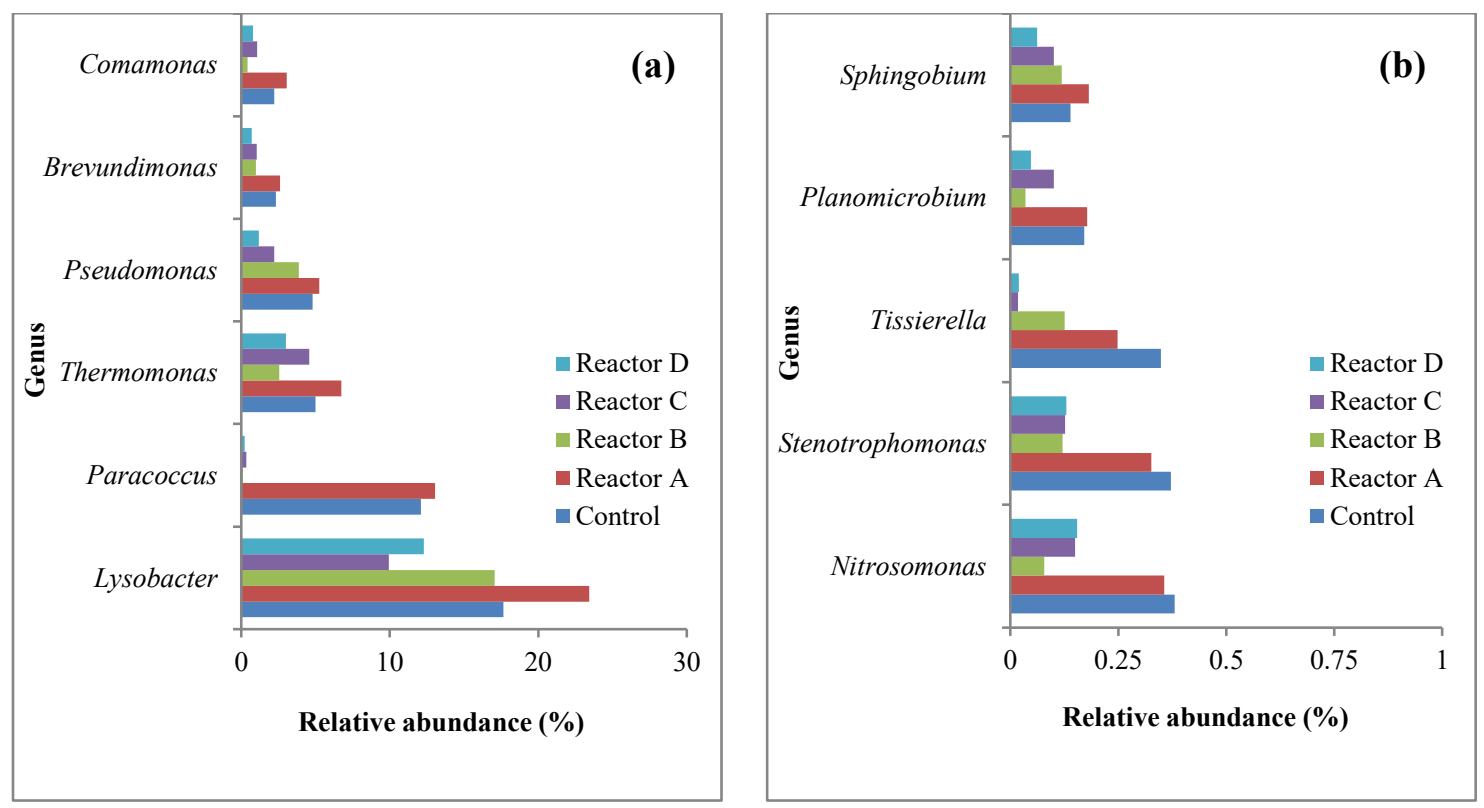

Figure 5-9 The average relative abundance of genera that decreased as a result of AgNPs and CuONPs presence; genera $\geq 1$ (a) and genera 0.1-1 (b). Reactors $A, B, C$, and $D$ are soil microcosms, which received anaerobically digested sludge with 2 mg AgNPs/g TS, 30 mg AgNPs/g TS, 2 mg CuONPs/g TS, and $30 \mathrm{mg}$ CuONPs /g TS, respectively. 


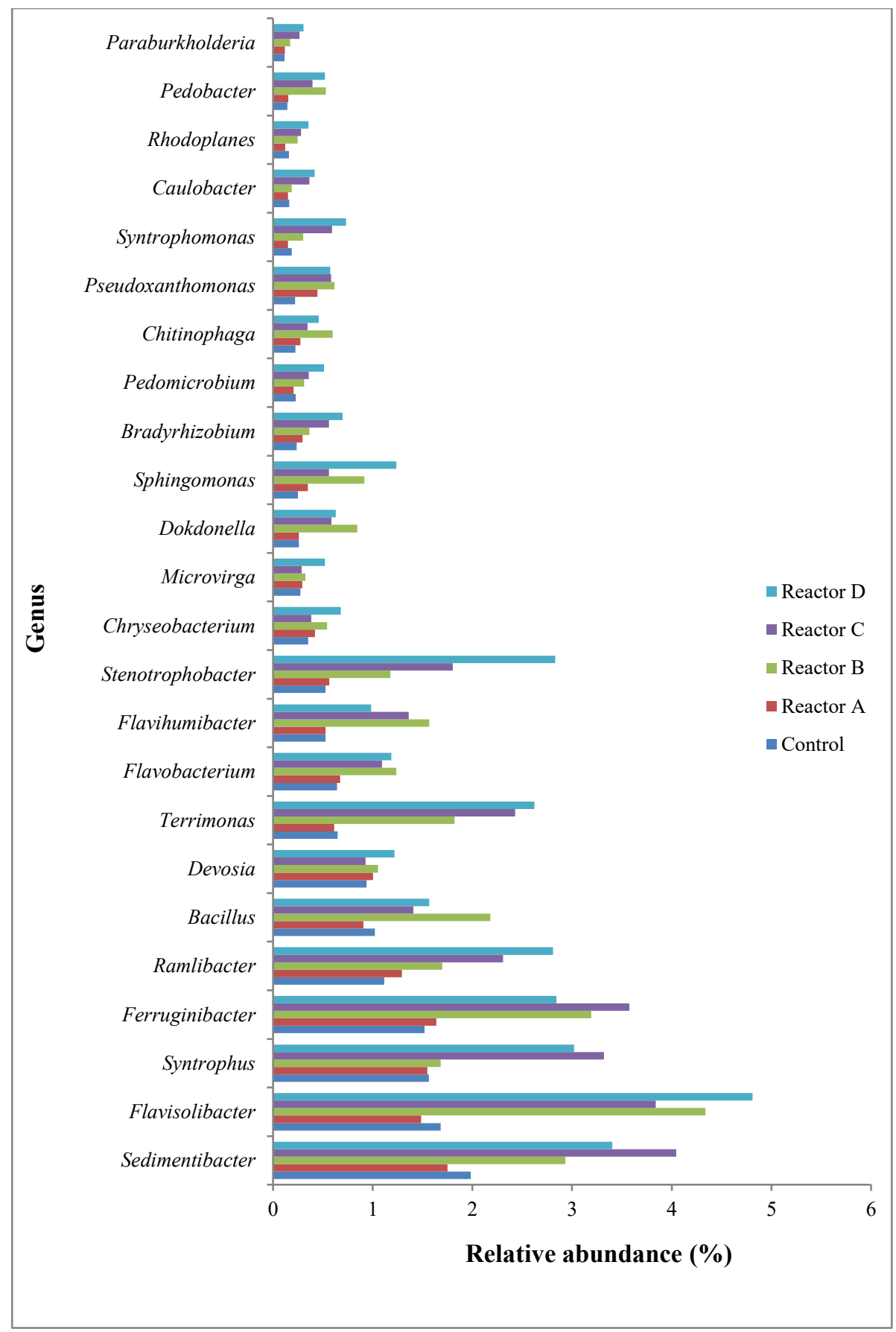

Figure 5-10 The average relative abundance of genera whose population increased as a result of the presence of AgNPs and CuONPs compared to the control. Reactors A, B, C, and D are soil microcosms, which received anaerobically digested sludge with 2 mg AgNPs/g TS, $30 \mathrm{mg}$ AgNPs/g TS, 2 mg CuONPs/g TS, and $30 \mathrm{mg} \mathrm{CuONPs} / \mathrm{g}$ TS, respectively. 


\subsubsection{Key bacteria potentially involved in soil functions}

The most important group of bacteria in soil is known as PGPR, which are associated with plant's roots and can directly promote healthy plant growth and enhance protection against deleterious rhizobacteria (DRB) (Rai \& Nabti, 2017). This is done through a variety of mechanisms, including atmospheric nitrogen fixation which makes it accessible to the plant, synthesis of siderophores that bind and transport iron so it is available to the plant root, solubilization of minerals such as phosphate and production of growth stimulating phytohormones (Rai \& Nabti, 2017; Pérez-Montaño et al., 2014). PGPR have also been reported to directly reduce the harmful effects of plant pathogens on crop yield by producing antibiotics (Rai \& Nabti, 2017). Based on a literature review, many genera that are considered PGPR can be involved in one or more beneficial biological activities in soil. Screening the top 117 abundant genera in this study, 21 different PGPR genera were identified according to the literature, as shown in Table S-1. These genera, their functions in soil, and their total relative abundance are listed in Table 5-4. 
Table 5-4 PGPR and their relative abundance in each soil reactor.

\begin{tabular}{|c|c|c|c|c|c|c|}
\hline Function & Phylum/Genera & $\begin{array}{l}\text { Control } \\
(\%)\end{array}$ & $\begin{array}{l}\text { Reactor } \\
\text { A } \\
(\%) \\
\end{array}$ & $\begin{array}{l}\text { Reactor } \\
\text { B } \\
(\%) \\
\end{array}$ & $\begin{array}{l}\text { Reactor } \\
\text { C } \\
(\%) \\
\end{array}$ & $\begin{array}{l}\text { Reactor } \\
\text { D } \\
(\%) \\
\end{array}$ \\
\hline $\begin{array}{l}\text { Atmospheric nitrogen } \\
\text { fixation }\end{array}$ & $\begin{array}{l}\text { Proteobacteria/ Nitrosospira, Rhizobium, } \\
\text { Mesorhizobium, Herbaspirillum, } \\
\text { Bradyrhizobium, Paraburkholderia and } \\
\text { Azotobacter } \\
\text { Firmicutes/ Bacillus and Paenibacillus. }\end{array}$ & 4.9 & 5.6 & 7.5 & 5.5 & 6.0 \\
\hline $\begin{array}{l}\text { Solubilization of } \\
\text { minerals such as } \\
\text { phosphate }\end{array}$ & $\begin{array}{l}\text { Proteobacteria/ Pseudomonas, } \\
\text { Rhizobium, Herbaspirillum, } \\
\text { Bradyrhizobium, Acinetobacter and } \\
\text { Azotobacter. } \\
\text { Firmicutes/ Bacillus and Paenibacillus, } \\
\text { Bacteroidetes/ Flavobacterium. }\end{array}$ & 8.2 & 9.4 & 9.1 & 7.0 & 6.1 \\
\hline $\begin{array}{l}\text { Suppression of plant } \\
\text { pathogens }\end{array}$ & $\begin{array}{l}\text { Proteobacteria/ Lysobacter, } \\
\text { Pseudomonas, Ochrobactrum and } \\
\text { Burkholderia. } \\
\text { Firmicutes/ Bacillus and Paenibacillus. }\end{array}$ & 24.1 & 24.5 & 23.6 & 14.3 & 15.7 \\
\hline $\begin{array}{l}\text { Production of } \\
\text { siderophores }\end{array}$ & $\begin{array}{l}\text { Proteobacteria/Acinetobacter and } \\
\text { Ochrobactrum. } \\
\text { Firmicutes/Bacillus, Paenibacillus. } \\
\text { Bacteroidetes/ Flavobacterium. }\end{array}$ & 2.3 & 2.3 & 3.9 & 3.3 & 3.4 \\
\hline $\begin{array}{l}\text { Synthesis of growth } \\
\text { stimulating } \\
\text { phytohormones }\end{array}$ & $\begin{array}{l}\text { Proteobacteria/ Xanthomonas, } \\
\text { Paracoccus, Pseudomonas, Rhizobium, } \\
\text { Acinetobacter, Azotobacter and } \\
\text { Azospirillum. } \\
\text { Firmicutes/ Bacillus. } \\
\text { Bacteroidetes/ Flavobacterium. } \\
\text { Actinobacteria / Arthrobacter. }\end{array}$ & 35.7 & 29.3 & 24.4 & 18.9 & 16.9 \\
\hline Bioremediation & $\begin{array}{l}\text { Proteobacteria/ Stenotrophomonas, } \\
\text { Pseudoxanthomona, Acinetobacter and } \\
\text { Burkholderia. } \\
\text { Firmicutes/ Bacillus. }\end{array}$ & 1.8 & 2.0 & 3.0 & 2.4 & 2.4 \\
\hline $\begin{array}{l}\text { Total relative } \\
\text { abundance of PGPR } \\
\text { (\%) }\end{array}$ & & 57.5 & 51.5 & 46.9 & 33.3 & 34.1 \\
\hline
\end{tabular}

A number of bacteria can accomplish atmospheric nitrogen-fixation by transforming atmospheric nitrogen into ammonia $\left(\mathrm{NH}_{3}\right)$ or ammonium $\left(\mathrm{NH}^{4+}\right)$ to make it available to plants through a special enzyme called nitrogenase (Newton, 2000; Monteiro et al., 2012; Pérez-Montaño et al., 2014; Gupta et al., 2015; Gano-Cohen et al., 2016; Rai \& Nabti, 2017). Several nitrogen-fixation genera were identified in this study, including 
Nitrosospira, Bacillus, Rhizobium, Paenibacillus, Mesorhizobium, Herbaspirillum, Bradyrhizobium, Paraburkholderia and Azotobacter. As shown in Table 5-4, the presence of AgNPs and CuONPs generally caused an increase in the total population of these genera, particularly in reactor B which had a higher concentration of AgNPs. Compared to the control, the increases were $14,53,11$ and $21 \%$ in reactors $\mathrm{A}, \mathrm{B}, \mathrm{C}$ and $\mathrm{D}$, respectively. This is likely due to the nitrogen-fixing bacteria, which produce extracellular polymeric substances, and thereby reducing the uptake of metal ions (Joshi \& Juwarkar, 2009; Yang et al., 2013).

Other genera have been reported to protect plants from various pathogens by activating defense genes (Keel, 2014; Lidbury et al., 2017). These genera were mentioned in the literature (Chakraborty et al., 2009; Pérez-Montaño et al., 2014; Keel, 2014 Gómez Expósito et al., 2015; Lidbury et al., 2017; Yang et al., 2017) and detected in the soil reactors, including Lysobacter, Pseudomonas, Bacillus, Paenibacillus, Ochrobactrum and Burkholderia. As seen in Table 5-4, AgNPs did not significantly impact the population of these genera. However, compared to the control, CuONPs affected the genera by decreasing their total population by 40.5 and $35 \%$ in reactors $C$ and $D$, respectively. Solubilization of phosphate is another function of PGPR. Phosphorus is an important building compound for the cell and is needed as a soluble form for plant uptake. However, the most available phosphorus forms in soil are insoluble (Souza et al., 2015). In the literature, several genera that solubilize phosphate and make it accessible to plants (Monteiro et al., 2012; Pérez-Montaño et al., 2014; Gupta et al., 2015; Rai \& Nabti, 2017) were identified in the soil reactors. These included Pseudomonas, Flavobacterium, Paenibacillus, Herbaspirillum and Acinetobacter. Their average relative abundance 
increased by 14 and $11 \%$ in reactors $\mathrm{A}$ and B and decreased by 15 and $25.5 \%$ in reactors $\mathrm{C}$ and $\mathrm{D}$ as shown in Table 5-4. This suggests that AgNPs promote the growth of these bacteria, and CuONPs inhibit their growth.

In addition, there is a group of bacteria known for their ability to clean contaminated soils through bioremediation (phytostimulation). This study identified a number of them that were reported in the literature (Hayward et al., 2010; Okhbakhsh-Zamin et al., 2011; Jeong et al., 2012; Radhakrishnan et al., 2017; Yang et al., 2017), including Bacillus, Stenotrophomonas, Pseudoxanthomona, Acinetobacter and Burkholderia. As shown in Table 5-4, AgNPs and CuONPs in the soil increased the average relative abundance of these genera by $10.50,65,33.5$ and $35 \%$ in reactors $\mathrm{A}, \mathrm{B}, \mathrm{C}$ and $\mathrm{D}$, respectively. Several genera can produce siderophores which are high-affinity iron-chelating compounds that bind $\mathrm{Fe}^{3+}$ ions to form soluble $\mathrm{Fe}^{3+}$ compounds that can easily move through the cell membrane and be taken up by plants (Gupta et al., 2015). Plants utilize iron in many different biological processes including nitrogen-fixing (Dixon \& Kahn, 2004) and respiration and photosynthesis (Kobayashi \& Nishizawa, 2012). In addition to the beneficial functions of Bacillus, Flavobacterium, Paenibacillus, Acinetobacter and Ochrobactrum mentioned above, these genera produce siderophores (Chakraborty et al., 2009; Babalola, 2010; Gupta et al., 2015; Grady et al., 2016; Rai \& Nabti, 2017). The total average relative abundance of the genera increased with higher concentrations of both AgNPs and CuONPs, as shown in Table 5-4. The increases compared to the control were $1.5,68,44$, and $49 \%$ in reactors A, B, C and D, respectively. This supports other studies that found the number of organisms that produce siderophores increased as the concentration of metals in the soil increased (Durenkamp et al., 2016). 
Many PGPR can produce phytohormones which have an essential role in plant growth and development. Phytohormones contribute to many functional mechanisms in plants, including, regulation of different physiological processes, increasing plant resistance to environmental factors, expression of genes and synthesis of enzymes, pigments, and metabolites (Tsavkelova et al., 2006; Babalola, 2010; Gupta et al., 2015). Several phytohormone producing bacteria were detected in the soil reactors during this study including, Xanthomonas, Paracoccus, Pseudomonas, Bacillus, Flavobacterium, Rhizobium, Acinetobacter, Azotobacter, Azospirillum and Arthrobacter, all of which can synthesize phytohormones, as frequently reported in the literature (Tsavkelova et al., 2006; Babalola, 2010; Pérez-Montaño et al., 2014; Rai \& Nabti, 2017). Due to AgNPs and CuONPs in the soil, the total relative abundance of these genera decreased in reactors A, B, C and D by $18,32,47$, and $53 \%$, respectively, compared to the control.

In general, AgNPs and CuONPs lowered the total average relative abundance of highly abundant genera from all PGPR discussed in this study. As shown in Table 5-4, the relative abundance of the 21 PGPR genera studied was $57.5 \%$ of the total genera population in the control, and this decreased by $10.5,18,42$ and $41 \%$ in reactors A, B, C and D, respectively. This indicated that CuONPs had a higher inhibitory impact on the PGPR.

\subsubsection{Distribution of PGPR genera over time}

Over time, the prevalence of PGPR genus OTUs followed different trends in the control and other soil reactors, with several showing significant differences in observed OTUs, including Lysobacter, Xanthomonas, Bacillus and Herbaspirillum. 
Lysobacter was the most abundant genus found in all the soil reactors (Figure 5-9), its OTUs increased and showed different distributions over time in each soil reactor, as shown in Figure 5-11(a). The highest increase was in reactor A, followed by the control and reactors $\mathrm{B}, \mathrm{D}$ and $\mathrm{C}$, respectively. Statistical analysis using T-test found significant differences between the control and reactors $\mathrm{C}$ and $\mathrm{D}$ ( $\mathrm{p}$-values $<0.05)$. The total average relative abundance followed the same pattern, with the highest in reactor A and the lowest in reactor $\mathrm{C}$ (Figure 5-9). The total average relative abundance was 17.67, 17.3, 17.06, 10 and $12.3 \%$ in the control and reactors $\mathrm{A}, \mathrm{B}, \mathrm{C}$ and $\mathrm{D}$, respectively, which suggests that CuONPs negatively affected Lysobacter by decreasing the population and reducing growth over time. This is of significant concern since Lysobacter represents $17.67 \%$ of the total bacterial population in the control and is also known to be significantly active against a wide range of pathogenic microorganisms in plants (Gómez Expósito et al., 2015).

The genus Xanthomonas was the second most abundant genus in all the soil reactors. Over time, Xanthomonas OTUs increased in all soil reactors as shown in Figure 5-11(b). The increase was less in the reactors with AgNPs and CuONPs, particularly those with lower concentrations of both nanoparticles (reactors A and C). There was a significant difference between the control and reactors $\mathrm{A}, \mathrm{C}$ and $\mathrm{D}$, and statistical analyses using T-test showed that p-values were $0.004,0.02$ and 0.03 for reactors $\mathrm{A}, \mathrm{C}$ and $\mathrm{D}$, respectively. This indicates that the lower concentration of AgNPs and both concentrations of CuONPs had an impact on the population of this genus. This is an issue, since Xanthomonas, is one of PGPR with the ability to produce phytohormones such as auxins and gibberellins (Tsavkelova et al., 2006), which play an essential role in regulating healthy plant growth (Tsavkelova et al., 2006; Cassán et al., 2015). The genus Xanthomonas contains many plant pathogenic 
species, including campestris, oryzae, populi, fragariae, albilineans and pv. pelargonii (Swings \& Civetta, 2012). However, these species had a very low average relative abundance, which was considered negligible in all the soil reactors during the study.

The Bacillus genus is involved in many PGPR functions in soil and plants, including atmospheric nitrogen fixation, solubilization of minerals, suppression of plant pathogens, production of siderophores and bioremediation (Babalola, 2010; Jeong et al., 2012; Souza et al., 2015; Rai \& Nabti, 2017; Radhakrishnan et al., 2017). As shown in Figure 5-11(c), after applying the sludge with nanoparticles, Bacillus OTUs decreased immediately by 70 , 81,36 , and $34 \%$ compared to the control in reactors $\mathrm{A}, \mathrm{B}, \mathrm{C}$ and $\mathrm{D}$, respectively, indicating that AgNPs have a greater impact on Bacillus than CuONPs. The Bacillus OTUs fluctuated over time in all soil reactors with lower OTUs in reactors A and C, which had lower concentrations of AgNPs and CuONPs, respectively, and higher OTUs in reactors B and D. This suggests that higher concentrations of AgNPs and CuONPs promote the growth of Bacillus, and this was confirmed by the average relative abundance of Bacillus that increased with higher concentrations of both nanoparticles, particularly CuONPs. The average relative abundances were $1.02,0.98,2.18,1.41$, and 1.57 in the control and reactors $\mathrm{A}, \mathrm{B}, \mathrm{C}$ and $\mathrm{D}$, respectively.

The OTUs of Herbaspirillum showed different observed OTU trends but generally increased in all reactors except between day 15 and 30 when it decreased. As shown in Figure 5-11(d), Herbaspirillum OTUs were impacted by the presence of both nanoparticles, particularly AgNPs, and statistical analyses using T-test, p-value $>0.05$ showed significant differences between the control and the other soil reactors. In addition, the average relative abundance of Herbaspirillum was $0.4,0.46,0.22,0.46$ and 0.46 in the 
control and reactors $\mathrm{A}, \mathrm{B}, \mathrm{C}$ and $\mathrm{D}$, respectively, and the average relative abundance decreased by $45.6 \%$ in reactor B compared to the control. Thus, higher concentrations of AgNPs had the greatest impact on the population of Herbaspirillum.
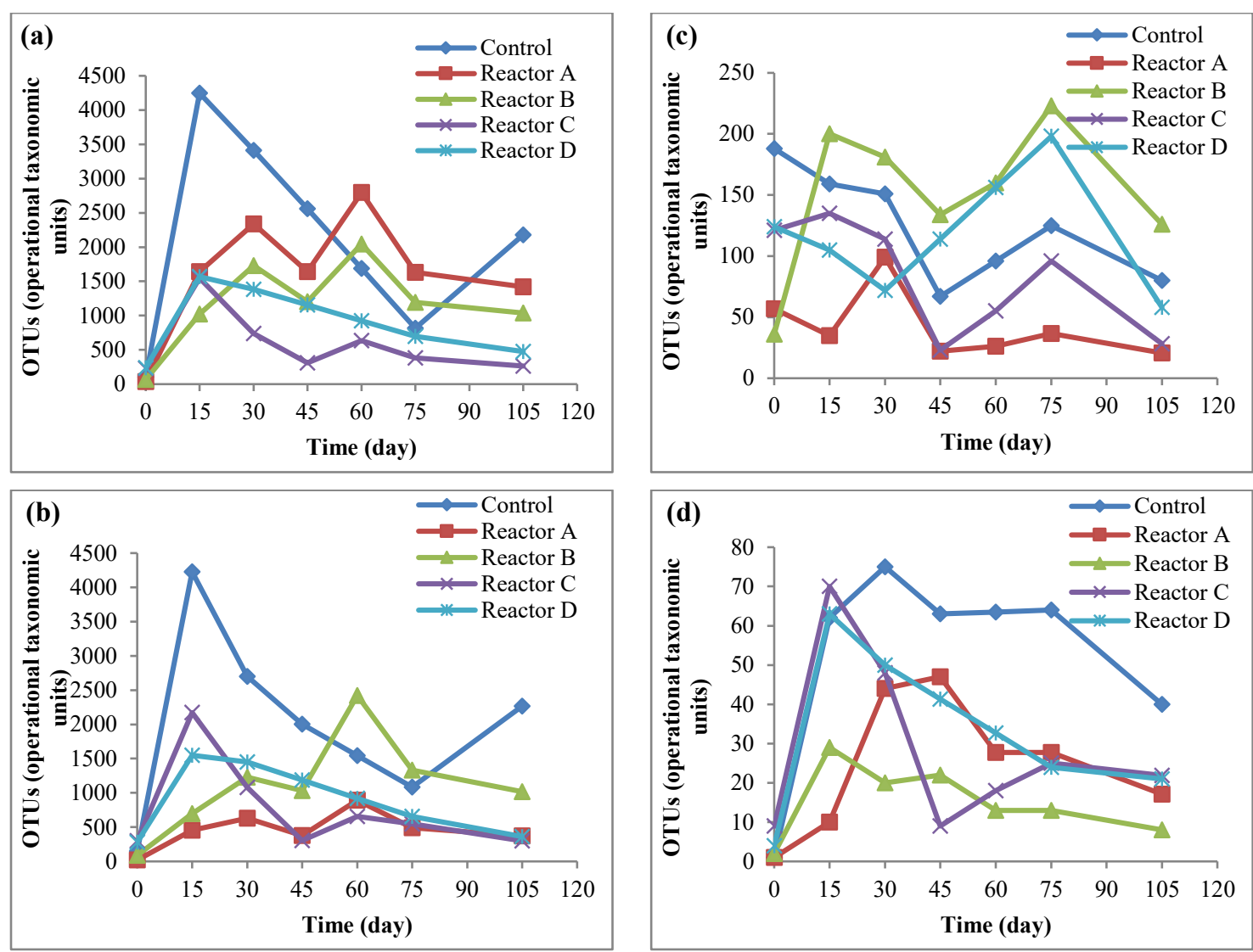

Figure 5-11 Distribution of the genera Lysobacter (a), Xanthomonas (b), Bacillus (c) and Herbaspirillum (d) over time. Reactors A, B, C, and D are soil microcosms, which received anaerobically digested sludge with $2 \mathrm{mg}$ AgNPs/g TS, $30 \mathrm{mg} \mathrm{AgNPs/g} \mathrm{TS,} 2$ mg CuONPs/g TS, and $30 \mathrm{mg} \mathrm{CuONPs/g}$ TS, respectively.

\subsubsection{General discussion on the impact of AgNPs and CuONPs on soil biological activities}

Soil bacterial population and diversity are good indicators of soil health due to their critical role in soil functions (Aislabie \& Deslippe, 2013). Nanoparticles in soil can potentially 
affect soil bacteria by altering their community composition, thereby impacting functions such as C, N and P cycling and disease resistance of plants (He et al., 2016; Asadishad et al., 2018; Grün et al., 2018). Hence, it is very important to assess the impacts and modifications nanoparticles are capable of. This is particularly important because significant amounts of nanoparticles, such as AgNPs, can remain in the soil matrix for long periods (He et al., 2019). Also, Durenkamp et al. (2016) reported that very low levels of AgNPs can leach from the soil and the remaining amount stays in the soil matrix.

This study examined bacterial community composition and their potential functions. In previous sections, it was discussed that AgNPs and CuONPs not only affect the bacterial composition but potential ecological functions as well. This was evident after assessing the changes in the HPC, alpha diversity represented by the Shannon-Wiener index, bacterial taxa OTUs, evenness and Beta-Euclidean diversity, and was confirmed by the negative impacts on PGPR. The changes in bacterial communities could be due to several different factors and mechanisms. For example, prolonged exposure to AgNPs can alter bacterial cell membrane lipids (Hachicho et al., 2014), and cause physiological changes in bacteria that can result in antibiotic resistance. Kaweeteerawat et al. (2017) reported how AgNPs inhibit Escherichia coli and cause oxidative stress, which eventually leads to increased bacterial resistance to antibiotics due to increased stress tolerance. Also, He et al. (2016) showed that AgNPs at three different concentrations (0.1,1 and $10 \mathrm{mg} / \mathrm{kg}$ soil) decreased soil microbial metabolic activity, nitrification potential and the abundances of ammoniaoxidizing bacteria. Another study by Asadishad et al. (2018) reported an inhibition effect of AgNPs and dissolving silver both at $100 \mathrm{mg} / \mathrm{kg}$ on selected enzymes in the soil, whereas CuONPs enhanced the enzymes. In addition, long-term effects of environmentally relevant 
concentrations of AgNPs ( 0.01 to $1 \mathrm{mg} \mathrm{AgNPs} / \mathrm{kg}$ soil) can instigate changes in microbial biomass and nitrogen cycling (Grün et al., 2018). During this study, CuONPs induced higher toxicity than AgNPs, which is likely due to the different toxicity principles, in addition to different characteristics of the nanoparticles, including particle size, shape and coating (Xiu et al. 2012). During this study, AgNPs were PVP coated while CuONPs were uncoated, which might impact the dissolution rate in the sludge. However, several studies, including Líbalová et al. (2018), showed that the dissolution of CuONPs in cell medium was slightly impacted by the surface modifications, and the CuONPs toxicity impact was a combination of the impact of the nanoparticles, their dissociation rate, and the toxicity of the coating agents.

Several researchers agree that AgNPs and CuONPs toxicity is mainly due to the rapid dissolution of nanoparticles, and the release of their ions into the soil (Cornelis et al., 2010; Shoults-Wilson et al., 2011; Xiu et al., 2012; Grün et al., 2018). In addition, a high concentration of divalent cations, including $\mathrm{Ca}^{2+}$ and $\mathrm{Mg}^{2+}$, can induce the dissolution process and cause the release of silver and copper ions from the nanoparticles ( $\mathrm{Li}$ et al., 2010). This can damage the cell membrane or produce reactive oxidative species (ROS) which reduce the bacterial population (Reidy et al., 2013). For example, Concha-Guerrero et al. (2014) showed that CuONPs at $160 \mathrm{mg} / \mathrm{L}$ can cause cell membrane degradation, including the presence of holes, cavities, cellar collapse and lyses. Nanoparticle toxicity can be due to several actions including the impact on bacterial basal respiration activity, and as reported by previous studies, the ability of AgNPs to reduce bacterial respiration (Kumar et al., 2014; Samarajeewa et al., 2017). Nanoparticle toxicity can also be caused by modifying a specific enzyme that controls nutrient cycling, such as the impact of AgNPs 
on dehydrogenase activity (Samarajeewa et al., 2017). Oliveira and Pampulha (2006) also reported the sensitivity of dehydrogenase activity to metal contamination.

This study found almost no correlation between TOC and HPC over time, as the results indicated the nanoparticles had no effect on TOC, but there was some effect on HPC. The results showed higher inhibition of HPC in the presence of CuONPs, as well, the high concentration of both AgNPs and CuONPs correlated to higher inhibition.

Many factors can alter or mitigate the nanoparticle impact, such as the physicochemical properties of soil, so they should be considered when evaluating the toxicity of nanoparticles to soil microorganisms (Shoults-Wilson et al., 2011). For example, high levels of organic matter and humic and fulvic acids can reduce the toxic effects of AgNPs and CuONPs (Dinesh et al., 2012). It is important to consider the toxicity of transformed nanoparticles and not only those that are pristine, as AgNPs and CuONPs are unstable under most environmental conditions and would likely transform once introduced into an ecosystem. For example, it is very likely that silver will react with sulfur, chloride and organic matter (Kramer et al., 2002), and recent studies suggest there is a significant amount of $\mathrm{Ag}_{2} \mathrm{~S}$ in most types of sludge, including aged sludge (Kim et al., 2010; Kaegi et al., 2013; Ma et al., 2013), as well as in soil (Pradas del Real et al., 2016). Combining AgNPs with other chemicals can alter their toxicity, such as, when reacting with sulfur, which is likely due to the formation of relatively insoluble $\mathrm{Ag}_{2} \mathrm{~S}$, reduces its toxicity (Levard et al., 2012). Thus, it is important to consider the impact of transformed AgNPs and CuONPs in the soil as they could be affected by various factors, including soil chemistry and natural organic matter (Tong et al., 2007; Chen et al., 2012). For example, 
the toxicity of AgNPs and CuONPs can be reduced by binding them to the soil organic matter, which can reduce their mobility in the soil matrix. In addition, natural colloidal and soil organic matters can cause AgNPs and CuONPs to aggregate into larger particles, which can reduce their antimicrobial activity.

\subsection{Conclusions}

This work examined the effects of AgNPs and CuONPs on soil bacterial communities through a realistic approach by adding the nanoparticles to wastewater sludge and allowing them to go through the sludge anaerobic digestion process before introducing them into the soil through biosolids land application. Bacterial growth and activity were affected by AgNPs and CuONPs and the results showed that both nanoparticles type and concentration can impact soil health factors. The changes can be positive or negative, depending on the nanoparticle type and dosage. Based on the results, the following conclusions were drawn:

1. The bacterial Shannon-Wiener index increased in the soil microcosms that received biosolids with AgNPs at $30 \mathrm{mg}$ AgNPs/g TS and CuONPs at 2 and $30 \mathrm{mg}$ CuONPs/g TS. Also, the observed OTUs of the most abundant phyla and genera (phyla with > $90 \%$ relative abundance and genera with $>70 \%$ relative abundance) decreased in these three soil microcosms. Conversely, the observed OTUs of the next most abundant phyla and genera (phyla with $\leq 10 \%$ relative abundance and genera with $\leq 30 \%$ relative abundance) increased with higher concentration. This indicates that the population of the highly abundant bacteria, which have an important role in soil, decreased, while the population of less important bacteria, in terms of soil function, thrived and increased. 
2. Proteobacteria was the most abundant phylum with approximately 62 to $81 \%$ of the total phyla population in all the soil microcosms. Compared to the soil that received biosolids with no nanoparticles, their relative abundance was decreased in the reactors that received biosolids with AgNPs at $30 \mathrm{mg}$ NPs/g TS and CuONPs at 2 and $30 \mathrm{mg}$ NPs/g TS by $8.44,17$ and $19 \%$, respectively.

3. The population of PGPR was reduced to $89,82,59$ and $60 \%$ in the microcosms that received biosolids with 2 and $30 \mathrm{mg}$ NPs/g TS for each of AgNPs and CuONPs, respectively compared to the control microcosm. Also, both nanoparticles significantly reduced HPC.

4. AgNPs and CuONPs significantly reduced the population of heterotrophic bacteria in the soil. In addition, they reduced the percentage of the live cells in the soil, but the reductions were not statistically significant.

This study evaluated the impact of AgNPs and CuONPs on soil bacterial community composition and activity, and how the effects varied depending on the nanoparticle type and concentration. As the impact on PGPR was also considered, the study provides a solid basis for further research. The study will be very useful for risk assessment of nanoparticle toxicity in biosolids amended agricultural land. 


\section{References}

Aislabie, J., \& Deslippe, J. R. (2013). Soil microbes and their contribution to soil services. In J. Dymond (Ed.), Ecosystem services in New Zealand-conditions and trends (pp. 143-161). Lincoln, New Zealand: Manaaki Whenua Press.

Alkorta, I., Aizpurua, A., Riga, P., Albizu, I., Amézaga, I., \& Garbisu, C. (2003). Soil enzyme activities as biological indicators of soil health. Reviews on Environmental Health, 18(1), $65-73$.

APHA, A. P., American water works Association, A., \& Water environment federation, W. (2017). Standard methods for the examination of water and wastewater (23 ed.). (E. Rice, R. Baird, \& A. Eaton, Eds.) Washington, DC, USA: APHA, American Public Health Association; American water works Association, AWWA; Water environment federation, WEF.

Asadishad, B., Chahal, S., Akbari, A., Cianciarelli, V., Azodi, M., Ghoshal, S., \& Tufenkji, N. (2018). Amendment of Agricultural Soil with Metal Nanoparticles: Effects on Soil Enzyme Activity and Microbial Community Composition. Environmental Science \& Technology, 52(4), 1908-1918.

Babalola, O. O. (2010). Beneficial bacteria of agricultural importance. Biotechnology Letters, 32(11), 1559-1570.

Blaser, S. A., Martin, S., Matthew, M., \& Konrad, H. (2008). Estimation of cumulative aquatic exposure and risk due to silver: contribution of nano-functionalized plastics and textiles. Science of The Total Environment, 390(2), 396-409.

Carbone, S., Antisari, L., Gaggia, F., Baffoni, L., Di Gioia, D., Vianello, G., \& Nannipieri, P. (2014). Bioavailability and biological effect of engineered silver nanoparticles in a forest soil. Journal of Hazardous Materials, 280, 89-96.

CCME, C. C. (2012). Guidance document for the beneficial use of municipal biosolids, municipal sludge and treated septage.

Chakraborty, U., Chakraborty, B. N., Basnet, M., \& Chakraborty, A. P. (2009). Evaluation of Ochrobactrum anthropi TRS-2 and its talc based formulation for enhancement of growth 
of tea plants and management of brown root rot disease. Journal of Applied Microbiology, 107(2), 625-634.

Chen, Y., Wang, D., Zhu, X., Zheng, X., \& Feng, L. (2012). Long-term effects of copper nanoparticles on wastewater biological nutrient removal and $\mathrm{N} 2 \mathrm{O}$ generation in the activated sludge process. Environmental Science \& Technology, 46(22), 12452-12458.

Colman, B. P., Arnaout, C. L., Anciaux, S., Gunsch, C. K., Hochella Jr, M. F., Kim, B., . . al., e. (2013). Low Concentrations of Silver Nanoparticles in Biosolids Cause Adverse Ecosystem Responses under Realistic Field Scenario. PLOS One, 8(2), e57189.

Colvin, V. L. (2003). The potential environmental impact of engineered nanomaterials. Nature Biotechnology, 21(10), 1166.

Concha-Guerrero, S. I., Brito, E. M., Piñón-Castillo, H. A., Tarango-Rivero, S. H., Caretta, C. A., Luna-Velasco, A., . . . Orrantia-Borunda, E. (2014). Effect of $\mathrm{CuO}$ nanoparticles over isolated bacterial strains from agricultural soil. Journal of Nanomaterials 2014 (2014): 206, 2014, 1-13.

Cornelis, G., Kirby, J. K., Beak, D., Chittleborough, D., \& McLaughlin, M. J. (2010). A method for determination of retention of silver and cerium oxide manufactured nanoparticles in soils. Environmental Chemistry, 7(3), 7298-308.

Currell, G., \& Dowman, A. (2005). Essential mathematics and statistics for science. Chichester;Hoboken, NJ: Wiley \& Sons.

Czárán, T. L., Hoekstra, R. F., \& Pagie, L. (2002). Chemical warfare between microbes promotes biodiversity. Proceedings of the National Academy of Sciences, 99(2), 786-790.

Dall'Agnol, R. F., Bournaud, C., Faria, S. M., Béna, G., Moulin, L., \& Hungria, M. (2017). Genetic diversity of symbiotic Paraburkholderia species isolated from nodules of Mimosa pudica (L.) and Phaseolus vulgaris (L.) grown in soils of the Brazilian Atlantic Forest (Mata Atlântica). FEMS Microbiology Ecology, 93(4), fix027.

Delgado-Baquerizo, M., Grinyer, J., Reich, P. B., \& Singh, B. K. (2016). Relative importance of soil properties and microbial community for soil functionality: insights from a microbial swap experiment. Functional Ecology, 30(11), 1862-1873. 
Dimkpa, C. O., McLean, J. E., Britt, D. W., \& Anderson, A. J. (2015). Nano-CuO and interaction with nano- $\mathrm{ZnO}$ or soil bacterium provide evidence for the interference of nanoparticles in metal nutrition of plants. Ecotoxicology, 24(1), 119-129.

Dinesh, R., Anandaraj, M., Srinivasan, V., \& Hamza, S. (2012). Engineered nanoparticles in the soil and their potential implications to microbial activity. Geoderma, 173, 19-27.

Dixon, R., \& Kahn, D. (2004). Genetic regulation of biological nitrogen fixation. Nature Reviews Microbiology, 2(8), 621.

Durenkamp, M., Pawlett, M., Ritz, K., Harris, J. A., Neal, A. L., \& McGrath, S. P. (2016). Nanoparticles within WWTP sludges have minimal impact on leachate quality and soil microbial community structure and function. Environmental Pollution, 211, 399-405.

Edgar, R. (2018). Usearch V11, Euclidean distance metric. Retrieved from drive5 Bioinformatics software and services: https://drive5.com/usearch/manual/euclidean_distance.html

Edwards, J. H., Thurlow, D. L., \& Ruf, M. E. (1992). Tillage and crop rotation effects on fertility status of a Hapludult soil. Soil Science Society of America Journal, 56(5), 1577-1582.

EPA, U. S. (2009). Targeted National Sewage Sludge Survey Sampling and Analysis Technical Report. United State : EPA.

Cassán, F. C., Okon, Y., \& Creus, C. M. (2015). Handbook for Azospirillum: Technical Issues and Protocols. New York: Springer.

Fernando, S., Gunasekara, T., \& Holton, J. (2018). Antimicrobial Nanoparticles: applications and mechanisms of action. Sri Lankan Journal of Infectious Diseases 8, no. 1 , 8(1), 2-11.

Frenk, S., Ben-Moshe, T., Dror, I., Berkowitz, B., \& Minz, D. (2013). Effect of metal oxide nanoparticles on microbial community structure and function in two different soil types. PLoS One, 8(12), e84441.

Gajjar, P., Pettee, B., Britt, D. W., Huang, W., Johnson, W. P., \& Anderson, A. J. (2009). Antimicrobial activities of commercial nanoparticles against an environmental soil microbe, Pseudomonas putida KT2440. Journal of Biological Engineering, 3(1), 9. 
Gano-Cohen, K. A., Stokes, P. J., Blanton, M. A., Wendlandt, C. E., Hollowell, A. C., Regus, J. U., . . Sachs, J. L. (2016). Non-nodulating Bradyrhizobium modulate the benefits of the legume-rhizobium mutualism. Applied and Environmental Microbiology, 82, 5259-5268.

Gattinger, A., Palojärvi, A., \& Schloter, M. (2008). Soil Microbial Communities and Related Functions. In Perspectives for Agroecosystem Management (pp. 279-292). Elsevier.

Gómez Expósito, R., Postma, J., Raaijmakers, J. M., \& Bruijn, I. D. (2015). Diversity and activity of Lysobacter species from disease suppressive soils. Frontiers in Microbiology, 6, 1243.

Gottschalk, F., Tobias, S., Roland W., S., \& Bernd, N. (2009). Modeled environmental concentrations of engineered nanomaterials ( $\mathrm{TiO} 2, \mathrm{ZnO}, \mathrm{Ag}, \mathrm{CNT}$, fullerenes) for different regions. Environmental Science \& Technology, 43(24), 9216-9222.

Grady, E. N., MacDonald, J., Liu, L., Richman, A., \& Yuan, Z.-C. (2016). Current knowledge and perspectives of Paenibacillus: a review. Microbial cell factories, 15(1 ), 203.

Grün, A.-L., Straskraba, S., Schulz, S., Schloter, M., \& Emmerling, C. (2018). Long-term effects of environmentally relevant concentrations of silver nanoparticles on microbial biomass, enzyme activity, and functional genes involved in the nitrogen cycle of loamy soil. Journal of Environmental Sciences, 69, 12-22.

Gupta, G., Parihar, S. S., Ahirwar, N. K., Snehi, S. K., \& Singh, V. (2015). Plant growth promoting rhizobacteria (PGPR): current and future prospects for development of sustainable agriculture. J Microb Biochem Technol, 7(2), 096-102.

Hachicho, N., Hoffmann, P., Ahlert, K., \& Heipieper, H. J. (2014). Effect of silver nanoparticles and silver ions on growth and adaptive response mechanisms of Pseudomonas putida mt2. FEMS Microbiology Letters, 355(1), 71-77.

Handy, R. D., Owen, R., \& Valsami-Jones, E. (2008). The ecotoxicology of nanoparticles and nanomaterials: current status, knowledge gaps, challenges, and future needs. Ecotoxicology , $17(5), 315-325$.

Hänsch, M., \& Emmerling, C. (2010). Effects of silver nanoparticles on the microbiota and enzyme activity in soil. Journal of Plant Nutrition and Soil Science, 173(4), 554-558. 
Hayward, A. C., Fegan, N., Fegan, M., \& Stirling, G. R. (2010). Stenotrophomonas and Lysobacter: ubiquitous plant-associated gamma-proteobacteria of developing significance in applied microbiology. Journal of Applied Microbiology, 108(3), 756-770.

He, J., Wang, D., \& Zhou, D. (2019). Transport and retention of silver nanoparticles in soil: Effects of input concentration, particle size and surface coating. Science of the Total Environment, 648, 102-108.

He, S., Feng, Y., Ni, J., Sun, Y., Xue, L., Feng, Y., . . Yang, L. (2016). Different responses of soil microbial metabolic activity to silver and iron oxide nanoparticles. Chemosphere, 147, 195-202.

Hong, C., Si, Y., Xing, Y., \& Li, Y. (2015). Illumina MiSeq sequencing investigation on the contrasting soil bacterial community structures in different iron mining areas. Environmental Science and Pollution Research, 22(14), 10788-10799.

Houba, V. J., Temminghoff, E. J., Gaikhorst, G. A., \& Vark, W. V. (2000). Soil analysis procedures using 0.01 M calcium chloride as extraction reagent. Communications in Soil Science and Plant Analysis, 31(9-10), 1299-1396.

Huang, H., Chen, Y., Yang, S., \& Zheng, X. (2019). CuO and ZnO nanoparticles drive the propagation of antibiotic resistance genes during sludge anaerobic digestion: possible role of stimulated signal transduction. Environmental Science, 6(2), 528-539.

Jeong, H., Jeong, D.-E., Kim, S. H., Song, G. C., Park, S.-Y., Ryu, C.-M., . . Choi, S.-K. (2012). Draft Genome sequence of the plant growth-promoting Bacterium Bacillus siamensis KCTC 13613T. Journal of Bacteriology, 194(15), 4148-4149.

Jnawali, A., Ojha, R., \& Marahatta, S. R. (2015). Role of Azotobacter in soil fertility and sustainability-A Review. Advances in Plants \& Agriculture Research, 2(6), 1-5.

Johansson, M., Stenberg, B., \& Torstensson, L. (1999). Microbiological and chemical changes in two arable soils after long-term sludge amendments. Biology and Fertility of Soils, 30(12), 160-167. 
Joshi, P. M., \& Juwarkar, A. A. (2009). In vivo studies to elucidate the role of extracellular polymeric substances from Azotobacter in immobilization of heavy metals. Environmental Science \& Technology, 43(15), 5884-5889.

Kabir, E., Kumar, V., Kim, K.-H., Yip, A. C., \& Sohn, J. (2018). Environmental impacts of nanomaterials. Journal of Environmental Management, 225, 261-271.

Kaegi, R., Voegelin, A., Ort, C., Sinnet, B., Thalmann, B., Krismer, J., . . Mueller, E. (2013). Fate and transformation of silver nanoparticles in urban wastewater systems. Water Research, 47(12), 3866-3877.

Kaegi, R., Voegelin, A., Sinnet, B., Zuleeg, S., Hagendorfer, H., Burkhardt, M., \& Siegrist, H. (2011). Behavior of metallic silver nanoparticles in a pilot wastewater treatment plant. Environmental Science \& Technology, 45(9), 3902-3908.

Kalińska, A., Jaworski, S., Wierzbicki, M., \& Gołębiewski, M. (2019). Silver and Copper Nanoparticles-An Alternative in Future Mastitis Treatment and Prevention? International Journal of Molecular Sciences, 20(7), 1672.

Kasana, R. C., Panwar, N. R., Kaul, R. K., \& Kumar, P. (2016). Copper nanoparticles in agriculture: biological synthesis and antimicrobial activity. In S. Ranjan, N. Dasgupta, \& E. Lichtfouse (Eds.), Nanoscience in Food and Agriculture (pp. 129-143). Springer, Cham.

Kaweeteerawat, C., Ubol, P. N., Sangmuang, S., Aueviriyavit, S., \& Maniratanachote, R. (2017). Mechanisms of antibiotic resistance in bacteria mediated by silver nanoparticles. Journal of Toxicology and Environmental Health, Part A 80(23-24), 1276-1289.

Keel, 1. (2014). Keel Lab. Retrieved January 24, 2019, from https://wp.unil.ch/keel-lab/ourresearch/pseudomonas-agriculture/

Kennedy, A., \& Smith, K. (1995). Soil microbial diversity and the sustainability of agricultural soils. Plant and soil, 170(1), 75-86.

Khodashenas, B. (2016). The Influential Factors on Antibacterial Behaviour of Copper and Silver Nanoparticles Behaviour of Copper and Silver Nanoparticles. Indian Chemical Engineer, 58(3), 224-239. 
Kibbee, R. J., \& Örmeci, B. (2017). Development of a sensitive and false-positive free PMA-qPCR viability assay to quantify VBNC Escherichia coli and evaluate disinfection performance in wastewater effluent. Journal of Microbiological Methods, 132, 139-147.

Kibblewhite, M., Ritz, K., \& Swift, M. (2007). Soil health in agricultural systems. Philosophical Transactions of the Royal Society B: Biological Sciences, 363(1492), 685-701.

Kiser, M. A., Ryu, H., Jang, H., Hristovski, K., \& Westerhoff, P. (2010). Biosorption of nanoparticles to heterotrophic wastewater biomass. Water Research, 44(14), 4105-4114.

Kobayashi, T., \& Nishizawa, N. K. (2012). Iron uptake, translocation, and regulation in higher plants. Annual Review of Plant Biology, 63, 131-152.

Kramer, J., Benoit, G., Bowles, K., DiToro, D., Herrin, R., Luther III, G., . . Shaw, J. (2002). Environmental chemistry of. In Silver in the Environment: Transport, Fate, and Effects. (pp. 1-25). Pensacola, FL, USA: SETAC.

Kumar, N., Palmer, G. R., Shah, V., \& Walker, V. K. (2014). The effect of silver nanoparticles on seasonal change in arctic tundra bacterial and fungal assemblages. PLoS One, 9(6), 99953.

Kumar, N., Shah, V., \& Walker, V. K. (2011). Perturbation of an arctic soil microbial community by metal nanoparticles. Journal of Hazardous Materials, 190(1-3), 816-822.

Kumar, N., Shah, V., \& Walker, V. K. (2012). Influence of a nanoparticle mixture on an arctic soil community. Environmental Toxicology and Chemistry, 31(1), 131-135.

Larney, F. J., \& Angers, D. A. (2012). The role of organic amendments in soil reclamation: a review. Canadian Journal of Soil Science, 92(1), 19-38.

Levard, C., Hotze, E. M., Lowry, G. V., \& Brown Jr., G. E. (2012). Environmental transformations of silver nanoparticles: impact on stability and toxicity. Environmental Science \& Technology, 46(13), 6900-6914.

Li, D., Qi, R., Yang, M., Zhang, Y., \& Yu, T. (2011). Bacterial community characteristics under long-term antibiotic selection pressures. Water Research, 45(18), 6063-6073.

Li, X., Lenhart, J. J., \& Walker, H. W. (2010). Dissolution-accompanied aggregation kinetics of silver nanoparticles. Langmuir, 26(22), 16690-16698. 
Lidbury, I. D., Murphy, A. R., Fraser, T. D., Bending, G. D., Jones, A. M., Moore, J. D., . . . Wellington, E. (2017). Identification of extracellular glycerophosphodiesterases in Pseudomonas and their role in soil organic phosphorus remineralisation. Scientific reports, 7(1), 2179.

Liu, G., Zhang, M., Jin, Y., Fan, X., Xu, J., Zhu, Y., . . Q Qian, H. (2017). The effects of low concentrations of silver nanoparticles on wheat growth, seed quality, and soil microbial communities. Water, Air, \& Soil Pollution, 228(9), 348.

Lourenco, K. S., Cassman, N. A., Pijl, A., Veen, J. A., Cantarella, H., \& Kuramae, E. E. (2018). Nitrosospira sp. govern nitrous oxide emissions in a tropical soil amended with residues of bioenergy crop. Frontiers in Microbiology, 9, 674.

Ma, R., Levard, C., Judy, J. D., Unrine, J. M., Durenkamp, M., Martin, B., . . Lowry, G. V. (2013). Fate of zinc oxide and silver nanoparticles in a pilot wastewater treatment plant and in processed biosolids. Environmental Science \& Technology, 48(1), 104-112.

Magurran, A. E. (1988). Ecological diversity and its measurement. Princeton university press.

McGee, C., Storey, S., Clipson, N., \& Doyle, E. (2017). Soil microbial community responses to contamination with silver, aluminium oxide and silicon dioxide nanoparticles. Ecotoxicology, 26(3), 449-458.

Monteiro, R. A., Brusamarello-Santos, L. C., Schmidt, M. A., Tadra-Sfeir, M. Z., Pankievicz, V., Cruz, L., . . . Pedrosa, F. (2012). Herbaspirillum-plant interactions: microscopical, histological and molecular aspects. Plant and Soil, 356(1-2), 175-196.

Mulder, C., Bazeley-White, E., D., P.G., H. A., Scherer-Lorenzen, M., \& Schmid, B. (2004). Species evenness and productivity in experimental plant communities. Oikos, 107(1), 5063.

Naz, I., Bano, A., \& Ul-Hassan, T. (2009). Isolation of phytohormones producing plant growth promoting rhizobacteria from weeds growing in Khewra salt range, Pakistan and their implication in providing salt tolerance to Glycine max L. African Journal of Biotechnology, $8(21), 5762-5766$. 
Newton, W. (2000). Nitrogen Fixation in Perspective. In F. Pedrosa, M. Hungria, G. Yates, \& W. Newton (Eds.), Nitrogen Fixation: From Molecules to Crop Productivity (pp. 3-8). Dordrecht: Springer.

Okhbakhsh-Zamin, F., Sachdev, D., Kazemi-Pour, N., Engineer, A., Pardesi, K. R., Zinjarde, S., . . . Chopade, B. A. (2011). Characterization of plant-growth-promoting traits of Acinetobacter species isolated from rhizosphere of Pennisetum glaucum. J Microbiol Biotechnol, 21(6), 556-566.

Oliveira, A., \& Pampulha, M. E. (2006). Effects of long-term heavy metal contamination on soil microbial characteristics. Journal of Bioscience and Bioengineering , 102(3), 157-161.

OME, O. M., \& OMAFR, O. M. (1996). Guidelines for The Utilization of Biosolid and Other Waste on Agricultural lands. Ontario, Canada: Ontario.

Oukarroum, A., Samadani, M., \& Dewez, D. (2014). Influence of pH on the toxicity of silver nanoparticles in the green alga Chlamydomonas acidophila. Water, Air, \& Soil Pollution, 225(8), 203.

Pandiarajan, J., Balaji, S., Mahendran, S., Ponmanickam, P., \& Krishnan, M. (2016). Synthesis and toxicity of silver nanoparticles. In S. Ranjan, N. Dasgupta, \& E. Lichtfouse (Eds.), Nanoscience in Food and Agriculture (pp. 73-98). Springer, Cham.

Pérez-Montaño, F., Alías-Villegas, C., Bellogín, R. A., Cerro, P. D., Espuny, M. R., JiménezGuerrero, I., . . . Cubo, T. (2014). Plant growth promotion in cereal and leguminous agricultural important plants: from microorganism capacities to crop production. Microbiological Research, 169(5-6), 325-336.

Pradas del Real, A. E., Castillo-Michel, H., Kaegi, R., Sinnet, B., Magnin, V., Findling, N., . . . Levard, C. (2016). Fate of Ag-NPs in sewage sludge after application on agricultural soils. Environmental Science \& Technology, 50(4), 1759-1768.

Pujalte, M., Lucena, T., Ruvira, M., Arahal, D., \& Macián, M. (2014). The Prokaryotes: Alphaproteobacteria and Betaproteobacteria. (pp. 460-463). Berlin, Heidelber: Springer. 
Radhakrishnan, R., Hashem, A., \& Abd_Allah, E. F. (2017). Bacillus: a biological tool for crop improvement through bio-molecular changes in adverse environments. Frontiers in Physiology, 8, 667.

Rai, A., \& Nabti, E. (2017). Plant Growth-Promoting Bacteria: Importance in Vegetable Production. In A. Zaidi, \& M. Khan (Eds.), Microbial Strategies for Vegetable Production (pp. 23-48). Springer, Cham.

Read, D. S., Matzke, M., Gweon, H. S., Newbold, L. K., Heggelund, L., Ortiz, M. D., . . Svendsen, C. (2016). Soil pH effects on the interactions between dissolved zinc, non-nano-and nano$\mathrm{ZnO}$ with soil bacterial communities. Environmental Science and Pollution Research, 23(5), 4120-4128.

Rebecca, B. (2004). Soil survey laboratory method manual. Soil Survey Laboratory Investigations Report.

Reidy, B., Haase, A., Luch, A., Dawson, K., \& Lynch, I. (2013). Mechanisms of silver nanoparticle release, transformation and toxicity: a critical review of current knowledge and recommendations for future studies and applications. Materials, 6(6), 2295-2350.

Samarajeewa, A., Velicogna, J., Princz, J., Subasinghe, R., Scroggins, R., \& Beaudette, L. (2017). Effect of silver nano-particles on soil microbial growth, activity and community diversity in a sandy loam soil. Environmental Pollution, 220, 504-513.

Shoults-Wilson, W. A., Reinsch, B. C., Tsyusko, O. V., Bertsch, P. M., Lowry, G. V., \& Unrine, J. M. (2011). Role of particle size and soil type in toxicity of silver nanoparticles to earthworms. Soil Science Society of America Journal, 75(2), 365-377.

Siller, H., Rainey, F. A., Stackebrandt, E., \& Winter, J. (1996). Isolation and characterization of a new gram-negative, acetone-degrading, nitrate-reducing bacterium from soil, Paracoccus solventivorans sp. nov. International Journal of Systematic and Evolutionary Microbiology, 46(4), 1125-1130.

Soltani, A.-A., Khavazi, K., Asadi-Rahmani, H., Omidvari, M., Dahaji, P. A., \& Mirhoseyni, H. (2010). Plant Growth Promoting Characteristics in Some Flavobacterium spp. Isolated from Soils of Iran. Journal of Agricultural Science, 2(4), 106-115. 
Souza, R. d., Ambrosini, A., \& Passaglia, L. M. (2015). Plant growth-promoting bacteria as inoculants in agricultural soils. Genetics and Molecular Biology, 38(4), 401-419.

Sudheer Khan, S., Kumar, E. B., Mukherjee, A., \& Chandrasekaran, N. (2011). Bacterial tolerance to silver nanoparticles (SNPs): Aeromonas punctata isolated from sewage environment. Journal of Basic Microbiology, 51(2), 183-190.

Sun, T. Y., Bornhöft, N. A., Hungerbühler, K., \& Nowack, B. (2016). Dynamic probabilistic modeling of environmental emissions of engineered nanomaterials. Environmental Science \& Technology, 50(9), 4701-4711.

Swings, J., \& Civetta, L. (2012). Xanthomonas (1st ed.). Hong Kong: Springer Science \& Business Media.

Tong, Z., Bischoff, M., Nies, L., Applegate, B., \& Turco, R. F. (2007). Impact of fullerene (C60) on a soil microbial community. Environmental Science \& Technology, 41(8), 2985-2991.

Tsavkelova, E., Klimova, S., Cherdyntseva, T., \& Netrusov, A. (2006). Microbial producers of plant growth stimulators and their practical use: a review. Applied Biochemistry and Microbiology, 42(2), 117-126.

Turco, R., Bischoff, M., Tong, Z., \& Nies, L. (2011). Environmental implications of nanomaterials: are we studying the right thing? Current Opinion in Biotechnology, 22(4), 527-532.

Upadhyay, S. K., Singh, J. S., Saxena, A. K., \& Singh, D. P. (2012). Impact of PGPR inoculation on growth and antioxidant status of wheat under saline conditions. Plant Biology, 14(4), $605-611$.

Wang, D., \& Chen, Y. (2016). Critical review of the influences of nanoparticles on biological wastewater treatment and sludge digestion. Critical Reviews in Biotechnology, 35(5), 816828.

Wang, R., Zhang, H., Sun, L., Qi, G., Chen, S., \& Zhao, X. (2017). Microbial community composition is related to soil biological and chemical properties and bacterial wilt outbreak. Scientific Reports , 7(1), 343. 
Whitley, A. R., Levard, C., Oostveen, E., Bertsch, P. M., Matocha, C. J., Kammer, F. v., \& Unrine, J. M. (2013). Behavior of Ag nanoparticles in soil: effects of particle surface coating, aging and sewage sludge amendment. Environmental Pollution, 182, 141-149.

Wu, B., Wang, Y., Lee, Y.-H., Horst, A., Wang, Z., Chen, D.-R., . . Tan, Y. J. (2010). Comparative eco-toxicities of nano- $\mathrm{ZnO}$ particles under aquatic and aerosol exposure modes. Environmental Science \& Technology, 44(4), 1484-1489.

Xiu, Z.-m., Zhang, Q.-b., Puppala, H. L., Colvin, V. L., \& Alvarez, P. J. (2012). Negligible particlespecific antibacterial activity of silver nanoparticles. Nano Letters, 8(12), 4271-4275.

Xu, C., Peng, C., Sun, L., Zhang, S., Huang, H., Chen, Y., \& Shi, J. (2015). Distinctive effects of $\mathrm{TiO} 2$ and $\mathrm{CuO}$ nanoparticles on soil microbes and their community structures in flooded paddy soil. Soil Biology and Biochemistry, 86, 24-33.

Yang, Y., Wang, J., Xiu, Z., \& Alvarez, P. J. (2013). Impacts of silver nanoparticles on cellular and transcriptional activity of nitrogen-cycling bacteria. Environmental Toxicology and Chemistry, 32(7), 1488-1494.

Yang, Y., Wang, N., Guo, X., Zhang, Y., \& Ye, B. (2017). Comparative analysis of bacterial community structure in the rhizosphere of maize by high-throughput pyrosequencing. PloS One, $12(5), 0178425$.

Zahran, H. H. (1999). Rhizobium-legume symbiosis and nitrogen fixation under severe conditions and in an arid climate. Microbiology and Molecular Biology, 63(4), 968-989. 


\section{Supplementary data}

\section{Total genomic DNA concentration}

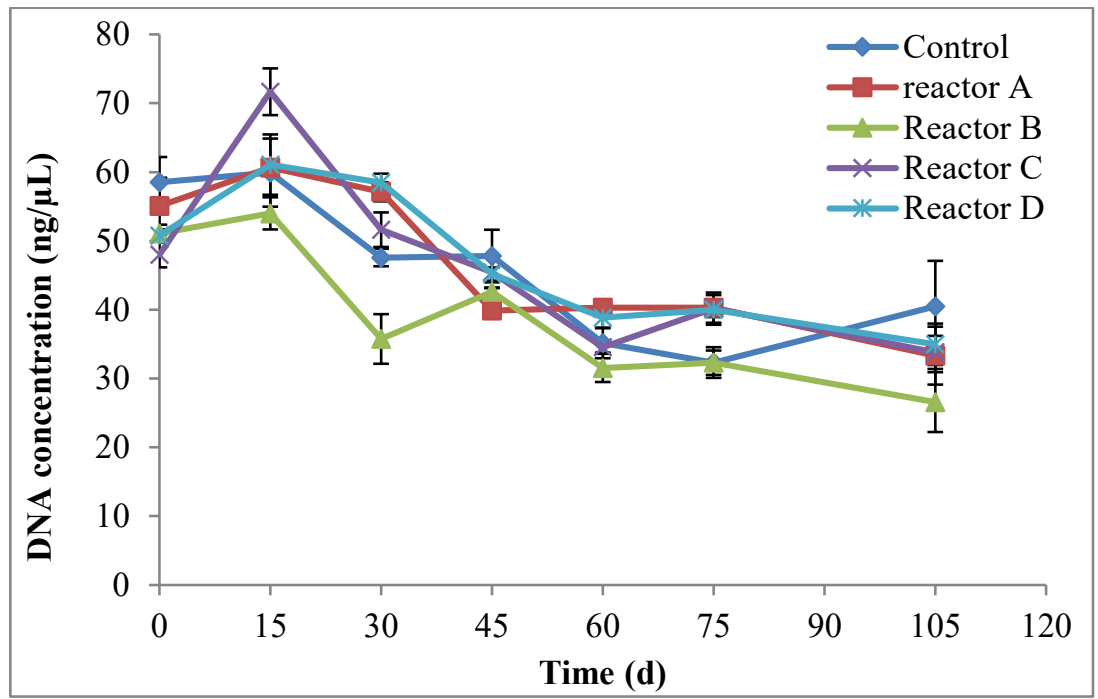

Figure S-1 total genomic concentration overtime. 


\section{The genera of interest and their role in soil}

\section{Table S-1 Genera of interest specifics.}

\begin{tabular}{|c|c|c|c|}
\hline Genus & Specification & Action in soil & Reference \\
\hline Lysobacter & $\begin{array}{l}\text { Gram-negative, non-motile and } \\
\text { aerobic bacteria. }\end{array}$ & -Disease suppression. & (Gómez Expósito et al., 2015) \\
\hline Xanthomonas & $\begin{array}{l}\text { Gram-negative, motile and } \\
\text { aerobic bacteria. }\end{array}$ & -Production of phytohormones. & (Tsavkelova et al., 2006) \\
\hline Paracoccus & $\begin{array}{l}\text { Gram-negative, non-motile and } \\
\text { aerobic bacteria. }\end{array}$ & $\begin{array}{l}\text {-Denitrification. } \\
\text {-Acetone degradation. }\end{array}$ & $\begin{array}{l}\text { (Siller et al., 1996; Pujalte et } \\
\text { al., 2014) }\end{array}$ \\
\hline Pseudomonas & $\begin{array}{l}\text { Gram-negative, motile and } \\
\text { aerobic bacteria. }\end{array}$ & $\begin{array}{l}\text {-Suppression of plants diseases. } \\
\text {-Solubilization of phosphate. } \\
\text {-Production of phytohormones. }\end{array}$ & $\begin{array}{l}\text { (Keel 2014; Lidbury et al., } \\
\text { 2017) }\end{array}$ \\
\hline Nitrosospira & $\begin{array}{l}\text { Gram-negative, motile and } \\
\text { aerobic bacteria. }\end{array}$ & $\begin{array}{l}\text {-Ammonia-oxidation. } \\
\text {-Nitrogen fixation. }\end{array}$ & $\begin{array}{l}\text { (Yang et al., 2017; Lourenco } \\
\text { et al., 2018) }\end{array}$ \\
\hline Bacillus & $\begin{array}{l}\text { Gram-positive, motile and } \\
\text { aerobic bacteria. }\end{array}$ & $\begin{array}{l}\text {-Plant disease suppression. } \\
\text {-Solubilization of phosphate. } \\
\text {-Nitrogen fixation. } \\
\text {-Phytostimulation. } \\
\text {-Siderophores production. } \\
\text {-Phytohormones production. }\end{array}$ & $\begin{array}{l}\text { (Jeong et al., 2012; } \\
\text { Radhakrishnan et al., 2017) }\end{array}$ \\
\hline Flavobacterium & $\begin{array}{l}\text { Gram-negative, motile and } \\
\text { aerobic bacteria }\end{array}$ & $\begin{array}{l}\text {-Phosphate solubilization. } \\
\text {-Siderophores production. } \\
\text {-Phytohormones production. }\end{array}$ & (Soltani et al., 2010) \\
\hline Rhizobium & $\begin{array}{l}\text { Gram-negative, motile and } \\
\text { aerobic bacteria. }\end{array}$ & $\begin{array}{l}\text {-Nitrogen fixation. } \\
\text {-Phytohormones production. }\end{array}$ & (Zahran, 1999) \\
\hline Paenibacillus & $\begin{array}{l}\text { Gram-negative, motile and } \\
\text { aerobic bacteria. }\end{array}$ & $\begin{array}{l}\text {-Plant disease suppression. } \\
\text {-Solubilization of phosphate. } \\
\text {-Nitrogen fixation. } \\
\text {-Siderophores production. }\end{array}$ & (Grady et al., 2016) \\
\hline Mesorhizobium & $\begin{array}{l}\text { Gram-negative, motile and } \\
\text { aerobic bacteria. }\end{array}$ & -Nitrogen fixation. & (Delgado et al., 2007) \\
\hline Herbaspirillum & $\begin{array}{l}\text { Gram-negative, motile and } \\
\text { aerobic bacteria. }\end{array}$ & $\begin{array}{l}\text {-Nitrogen fixation, } \\
\text {-Phosphate solubilization. }\end{array}$ & (Monteiro et al., 2012) \\
\hline Stenotrophomonas & $\begin{array}{l}\text { Gram-negative, motile and } \\
\text { aerobic bacteria. }\end{array}$ & $\begin{array}{l}\text {-Bioremediation of hydrocarbon- } \\
\text { contaminated soils. }\end{array}$ & (Hayward et al., 2010) \\
\hline Bradyrhizobium & $\begin{array}{l}\text { Gram-negative, motile and } \\
\text { aerobic bacteria. }\end{array}$ & -Nitrogen fixation. & (Gano-Cohen et al., 2016) \\
\hline Pseudoxanthomona & & $\begin{array}{l}\text {-Bioremediation of hydrocarbon- } \\
\text { contaminated soils. }\end{array}$ & \\
\hline Acinetobacter & $\begin{array}{l}\text { Gram-negative, non-motile and } \\
\text { aerobic bacteria. }\end{array}$ & $\begin{array}{l}\text {-Phosphate solubilization. } \\
\text {-Bioremediation of hydrocarbon- } \\
\text { contaminated soils. } \\
\text {-Siderophores production. } \\
\text {-Phytohormones production. }\end{array}$ & $\begin{array}{l}\text { (Okhbakhsh-Zamin et al., } \\
\text { 2011) }\end{array}$ \\
\hline Paraburkholderia & $\begin{array}{l}\text { Gram-negative, motile and } \\
\text { aerobic bacteria. }\end{array}$ & -Nitrogen fixation. & (Dall'Agnol et al., 2017) \\
\hline Ochrobactrum & $\begin{array}{l}\text { Gram-negative, motile and } \\
\text { aerobic bacteria. }\end{array}$ & $\begin{array}{l}\text {-Plant disease suppression. } \\
\text {-Siderophore production. }\end{array}$ & (Chakraborty et al., 2009) \\
\hline Burkholderia & $\begin{array}{l}\text { Gram-negative, motile and } \\
\text { aerobic bacteria. }\end{array}$ & $\begin{array}{l}\text {-Bioremediation (degradation of } \\
\text { aromatic compounds). } \\
\text {-Plants disease suppression. }\end{array}$ & (Yang et al., 2017) \\
\hline Azotobacter & $\begin{array}{l}\text { Gram-negative, motile and } \\
\text { aerobic bacteria. }\end{array}$ & $\begin{array}{l}\text {-Nitrogen fixation. } \\
\text {-Phytohormones production. }\end{array}$ & (Jnawali et al., 2015) \\
\hline Azospirillum & $\begin{array}{l}\text { Gram-negative, motile and } \\
\text { microaerophilic bacteria. }\end{array}$ & $\begin{array}{l}\text { - Nitrogen fixation. } \\
\text {-Phytohormones production. }\end{array}$ & (Cassán et al., 2015) \\
\hline Arthrobacter & $\begin{array}{l}\text { Gram-variability, motile and } \\
\text { aerobic bacteria. }\end{array}$ & -Phytohormones production. & $\begin{array}{l}\text { (Naz et al., 2009; Upadhyay et } \\
\text { al., 2012) }\end{array}$ \\
\hline
\end{tabular}



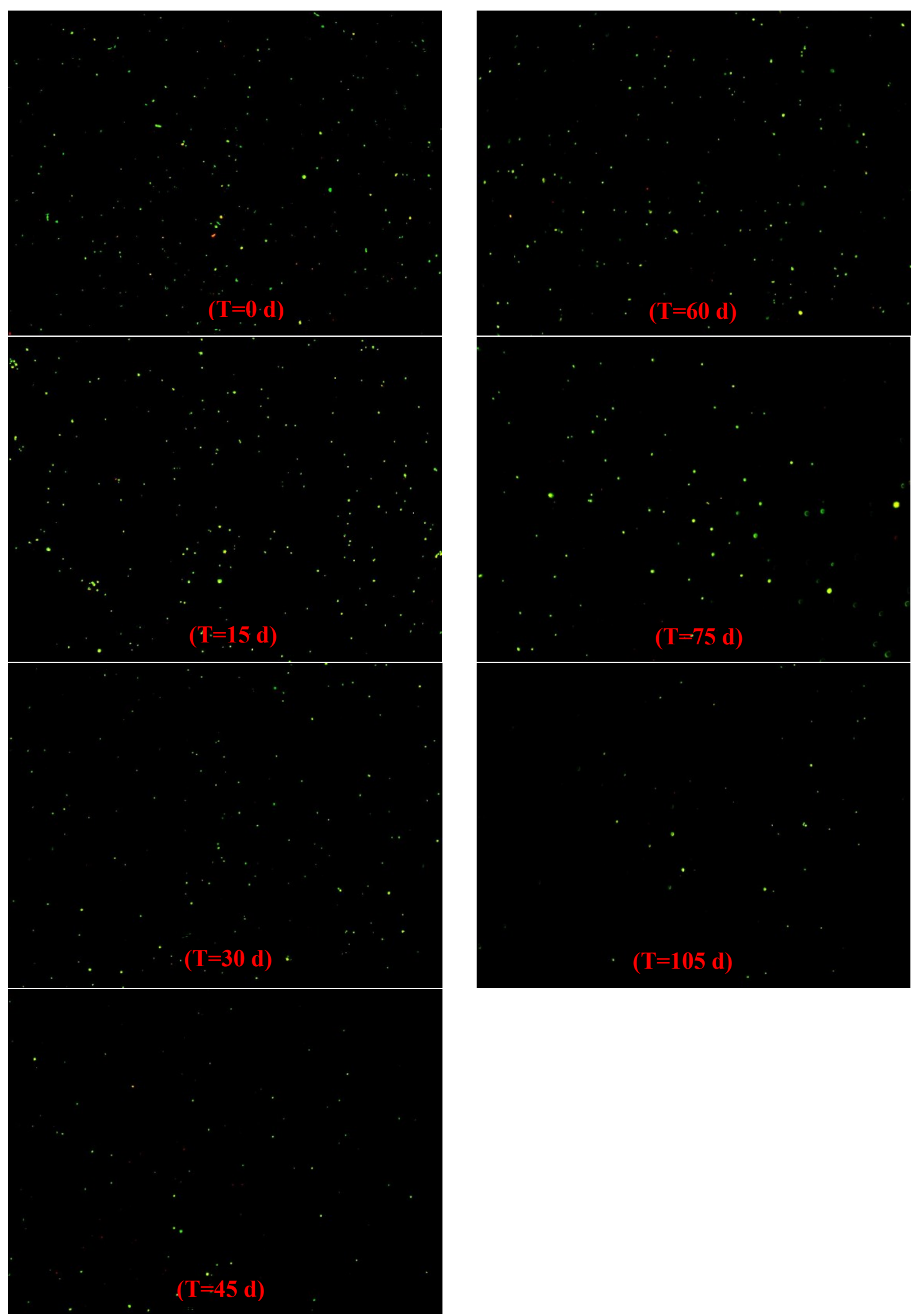

Figure S-2 Live/Dead cells images of the control over time. 


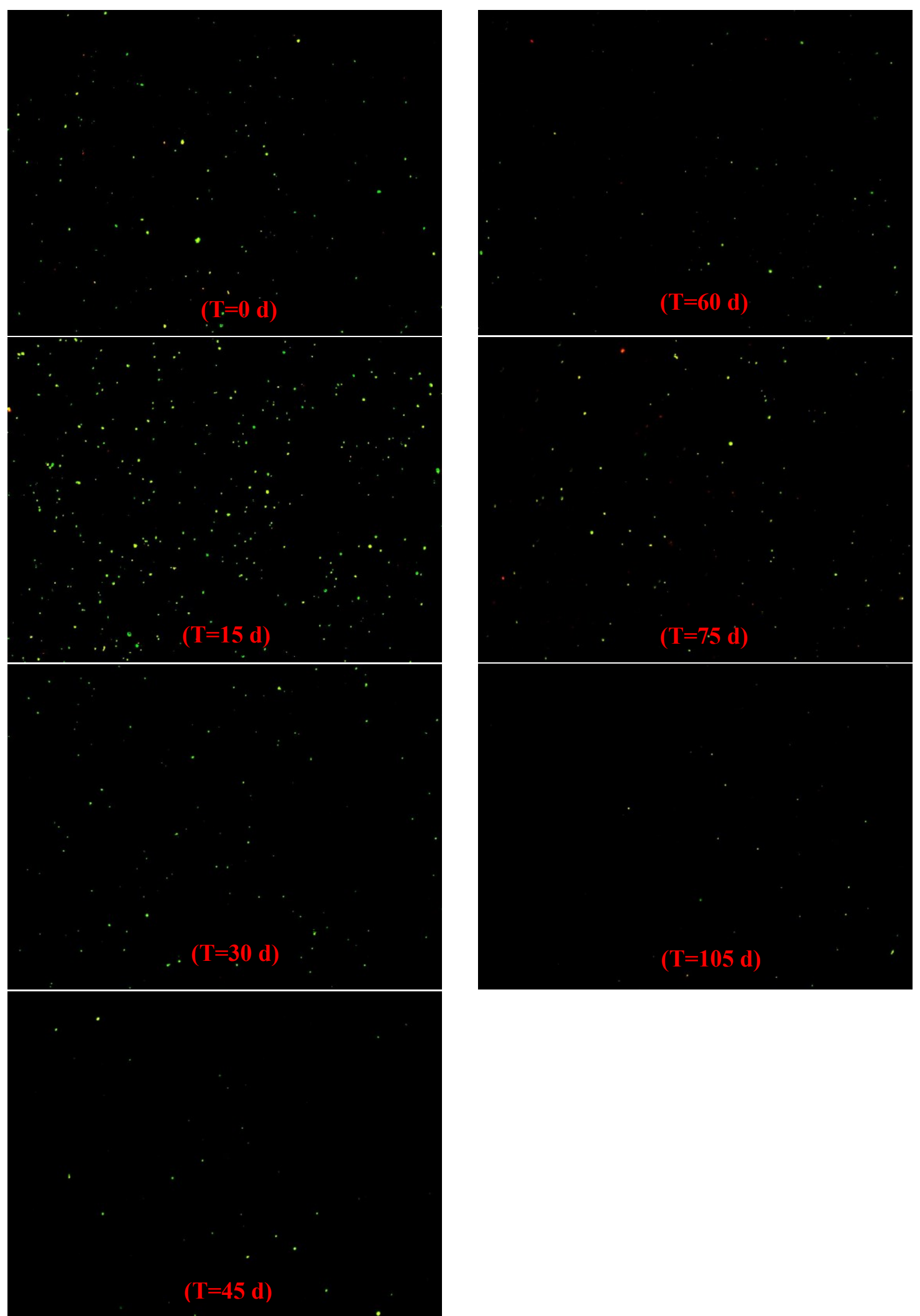

Figure S-3 Live/Dead cells images of reactor A over time. 


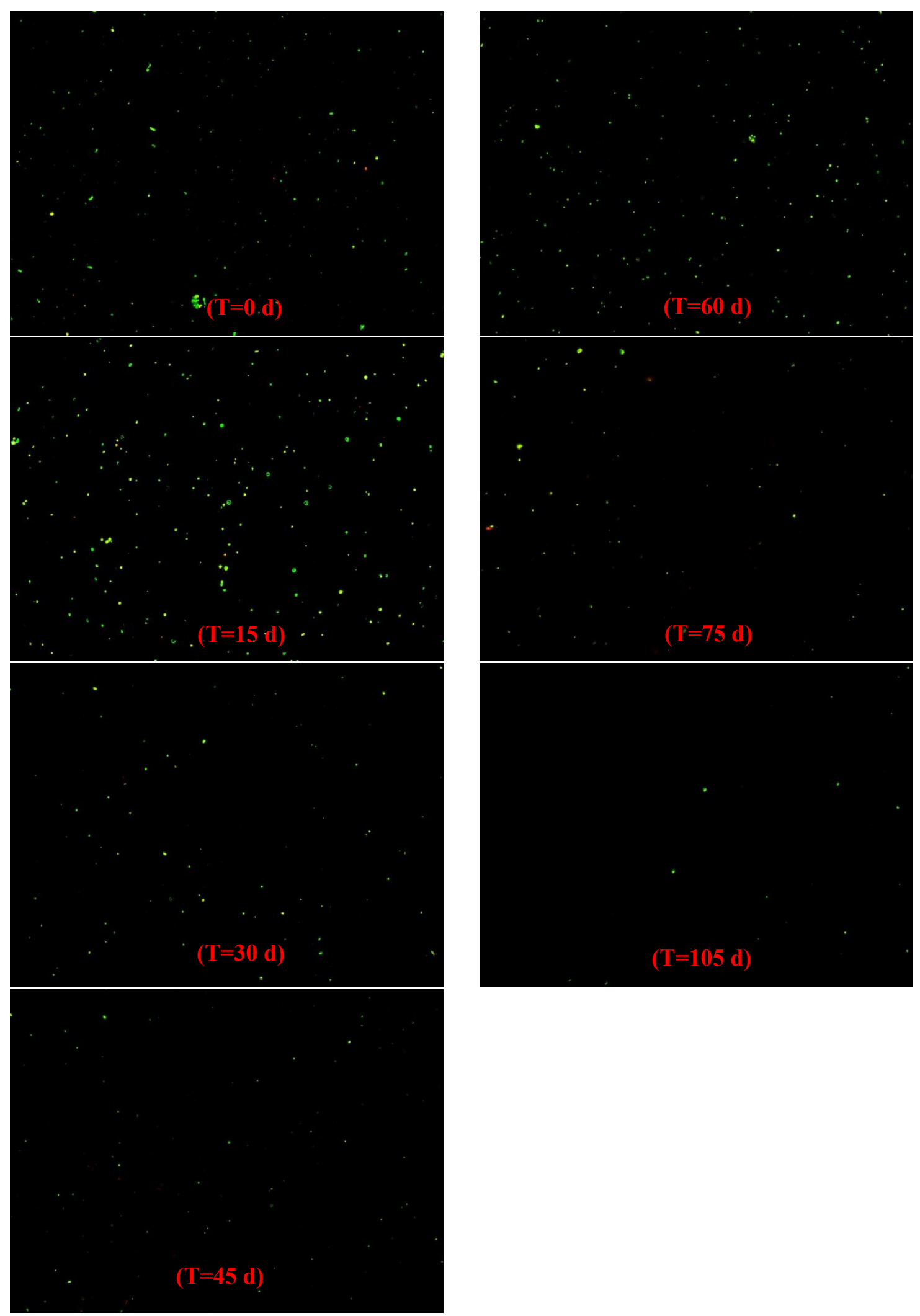

Figure S-4 Live/Dead cells images of reactor B over time. 


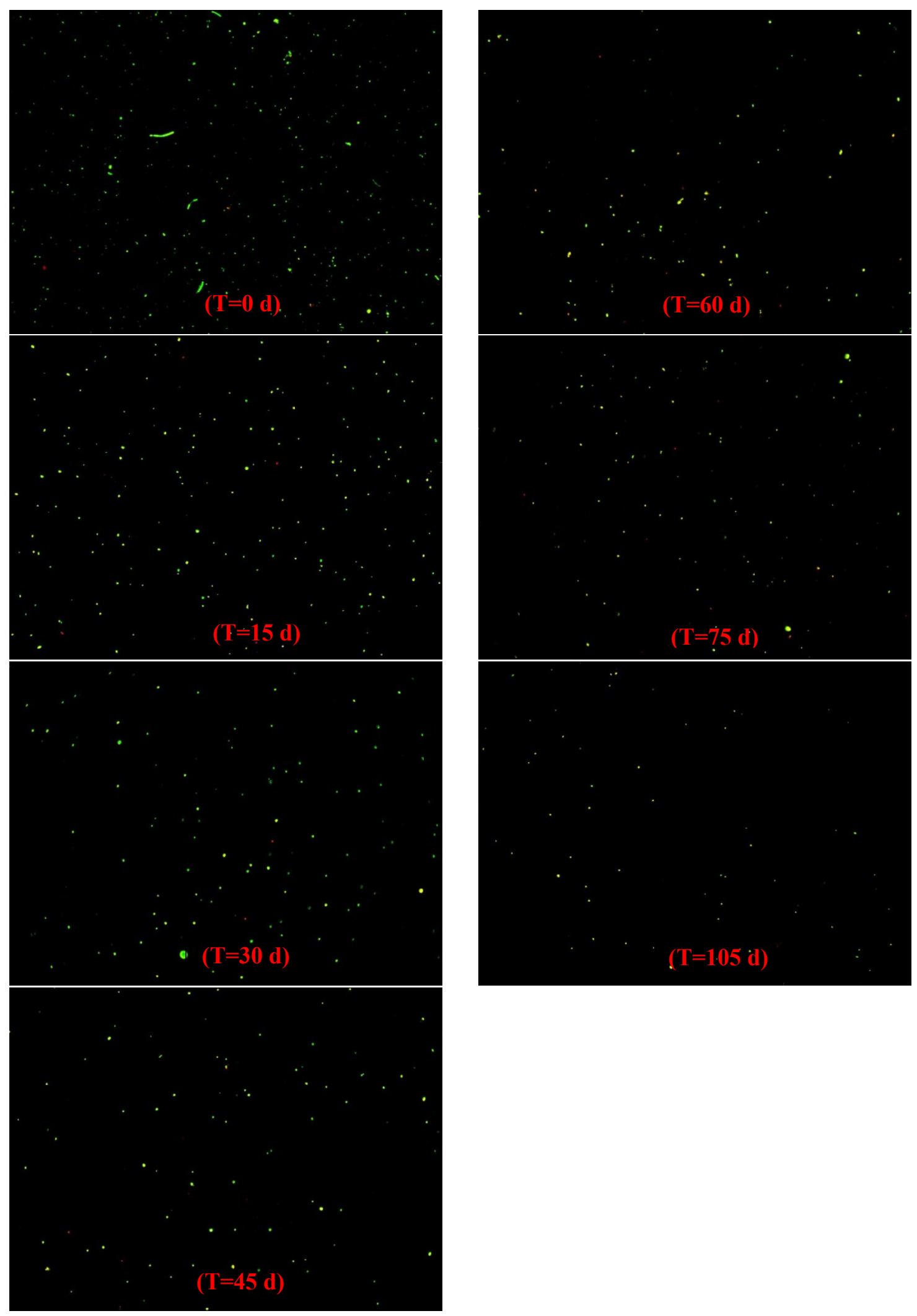

Figure S-5 Live/Dead cells images of reactor $\mathrm{C}$ over time. 


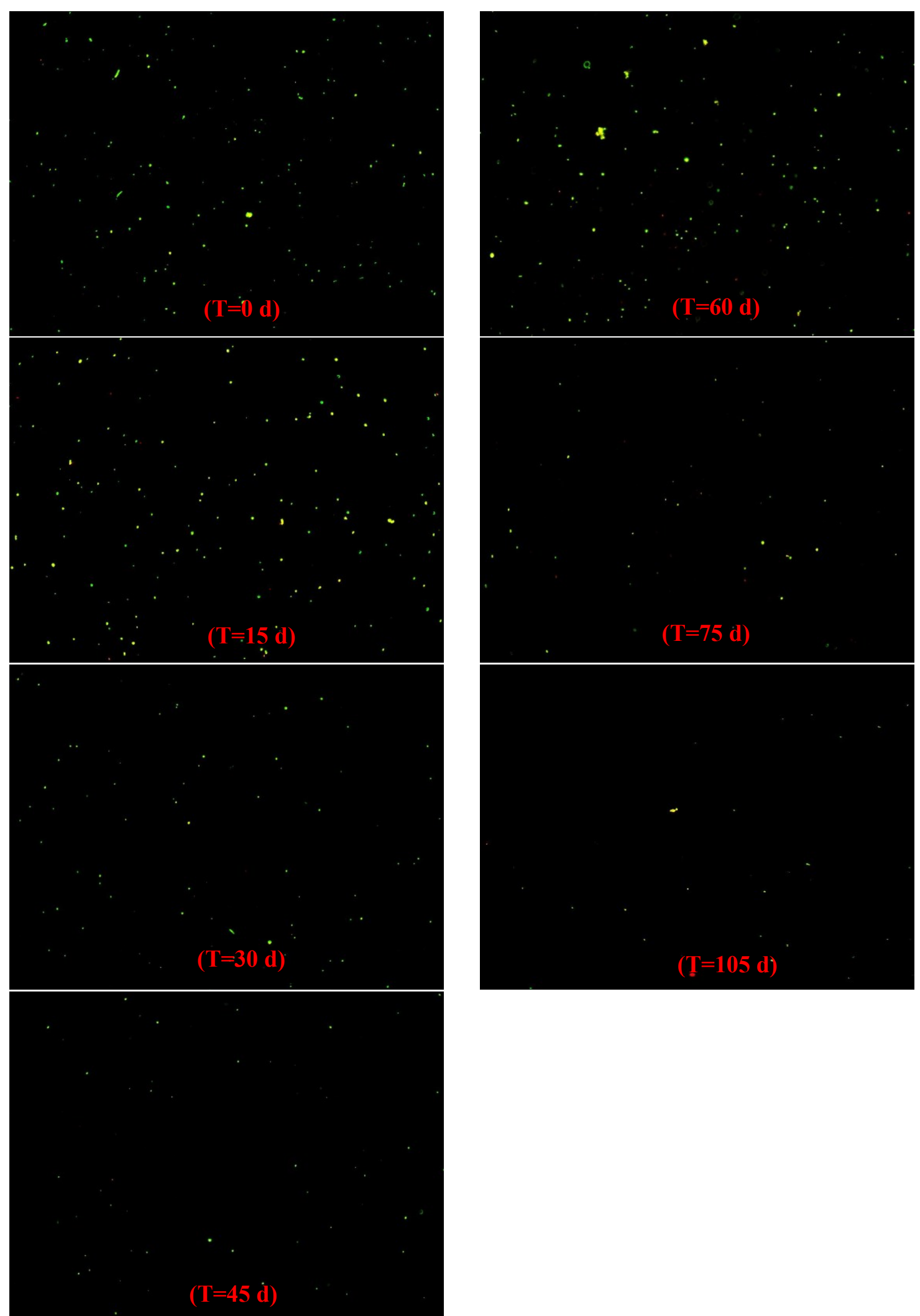

Figure S-6 Live/Dead cells images of reactor D over time. 


\title{
Chapter 6: Transformation and Removal of Silver Nanoparticles during Chemical Conditioning of Sludge and Their Impact on Soil Bacteria after Land Application
}

\begin{abstract}
The growing use of silver nanoparticles (AgNPs) in personal care products and clothing has increased their concentration in wastewater, and subsequently in sludge. Many studies have found that significant amounts of nanoparticles entering wastewater ultimately end up in sludge, raising concerns about their state transformation and toxicity, both during treatment processes and after land application of sludge. This research investigated the transformation and removal of AgNPs during chemical conditioning and their impact on soil microorganisms after land application. Ferric chloride $\left(\mathrm{FeCl}_{3}\right)$, aluminum sulfate $\left(\mathrm{Al}_{2}\right.$ $\left(\mathrm{SO}_{4}\right)_{3} \bullet(14-18) \mathrm{H}_{2} \mathrm{O}$ ), and synthetic polymer (polyacrylamide) were used as conditioning chemicals, and they all effectively removed AgNPs from the liquid phase and concentrated them in sludge solids. However, the floc structures and the incorporation of nanoparticles were different for different conditioners. Transmission electron microscopy (TEM) and energy-dispersive x-ray spectroscopy (EDS) images showed that AgNPs went through physical, chemical and morphological changes in sludge that were not observed in nanopure water. Using single particle-inductively coupled plasma-mass spectroscopy (SPICP-MS) and ICP-MS, distribution of dissolved and nanoparticle silver was investigated in the liquid phase. The results showed negligible quantities of AgNPs remaining in the liquid phase after chemical conditioning of sludge. After chemical conditioning, sludge samples with and without AgNPs were applied on soil and the impact on soil bacteria was
\end{abstract}


investigated over three months using qPCR, heterotrophic plate count, and Live/Dead viability staining. No significant impact of AgNPs, at a concentration of $20 \mathrm{mg} \mathrm{AgNPs} / \mathrm{kg}$ soil, was observed on heterotrophic soil bacteria and the five assessed bacterial phyla (Acidobacteria, Actinobacteria, Bacteroidetes, Firmicutes and Proteobacteria) in soil. However, the type of chemicals used for sludge conditioning appeared to have an impact on some of the bacterial phyla in soil, but the impact was not statistically significant.

Keywords: silver nanoparticles, sludge, chemical conditioning, land application, bacterial phyla

\subsection{Introduction}

Silver nanoparticles (AgNPs) have received particular attention in recent years due to their wide use as antimicrobial agents in commercial products and consequently their higher concentrations in the environment compared to other nanoparticles (Vance et al., 2015). Many studies conducted under controlled conditions showed the potential toxicity of AgNPs on different aquatic and terrestrial organisms, including plants, algae, fungi, fish, earthworms and bacteria (Colman et al., 2013; Cunningham et al., 2013; Panacek et al., 2009; Shoults-Wilson et al., 2011; Masrahi et al., 2014). AgNPs and other nanoparticles used in commercial products (e.g., cosmetics, sunscreen, shampoo, disinfectants, textiles, laundry products), ultimately end up in sewers (Benn et al., 2010; Geranio et al., 2009; Benn \& Westerhoff, 2008). Many studies have shown that significant amounts of AgNPs entering wastewater streams accumulate in biosolids (sludge) at the end of treatment (Schlich et al., 2013; Kaegi et al., 2011; Shafer et al., 1998). This raises concerns about not 
only the impact of AgNPs on sludge treatment processes but also their impact on soil ecosystem after application of biosolids to agricultural lands.

Chemical conditioning is one of the primary sludge treatment processes and is necessary to improve the solid-liquid separation before dewatering (Metcalf \& Eddy, 2003). Chemical conditioners typically have a high molecular weight and high charge, they are added in high concentrations and thus can have a major impact on the chemistry, morphology, fate and behavior of nanoparticles. There are research gaps on the fate and impact of nanoparticles during sludge treatment processes, and existing studies have largely focused on biological treatment processes and very little information is available on chemical treatment processes such as sludge conditioning. Lombi et al. (2013), Ma et al. (2013) and Impellitteri et al. (2013) investigated the status of AgNPs during anaerobic digestion, composting, and incineration and reported that most of the initial AgNPs were converted to $\mathrm{Ag}_{2} \mathrm{~S}$, and that several factors such as surface coating, $\mathrm{AgNPs}$ characteristics, and treatment conditions played a role. Sludge conditioning and the impact of the type of sludge conditioners used still remain to be investigated.

Land application of biosolids is the main sludge disposal process in many countries (Shammas \& Wang, 2007; Metcalf \& Eddy, 2003) followed by incineration. Large quantities of biosolids are produced daily by wastewater treatment plants, and treated sludge can find beneficial use and provide a means to recycle nutrients and organic carbon. For example, the 390 million litres of wastewater processed per day by the city of Ottawa produces approximately 39 dry tons of biosolids that are used as soil fertilizer (City of Ottawa, 2014). Land application of biosolids is a useful approach to improve soil nutrients such as carbon and nitrogen; however, it can add unwanted metals (Johansson et al., 1999), 
including AgNPs to the soil (Blaser et al., 2008). Studies have shown that AgNPs, which are typically used as antibacterial agents, could also impact soil microbial activities (Colman et al., 2013; Samarajeewa et al., 2017) at high concentrations. This is a concern, as soil organisms are responsible for organic matter decomposition and nutrient cycling.

Several studies that investigated the impact of AgNPs on soil biological activity after applying biosolids found high toxicity in soil microbial communities (Kumar et al., 2011; Colman et al., 2013; Schlich et al., 2016). The concentrations of AgNPs used in these studies varied widely and they were typically much higher than environmentally relevant concentrations. In addition, previous studies largely used virgin AgNPs and not those that have already undergone treatment processes, which would impact their behavior and impact. The main objective of this study was to investigate the fate, transformation and removal of AgNPs during chemical conditioning of sludge with a synthetic polymer, ferric chloride and alum, and determine the impact of AgNPs that went through conditioning as well as the conditioning chemicals on soil microorganisms after application of sludge.

\subsection{Materials and methods}

\subsubsection{AgNPs, sludge and soil characteristics and preparation}

Pristine silver nanoparticles used in this study, suspended in water at a $99.9 \%$ purity, properties shown in Table 6-1, were purchased from nanoComposix Inc. (San Diego, CA, USA). The particle size distribution of AgNPs was examined and confirmed by transmission electron microscopy (TEM) analysis, and the AgNPs suspension stock solution was stored at $4^{\circ} \mathrm{C}$ until use. 
Table 6-1 AgNPs characteristics.

\begin{tabular}{|l|l|}
\hline Diameter (TEM) & $23.1 \pm 6.9 \mathrm{~nm}$ \\
\hline Coefficient of variation & $29.8 \%$ \\
\hline Surface area (TEM) & $21.5 \mathrm{~m}^{2} / \mathrm{g}$ \\
\hline Particle concentration & $7.2 \times 10^{13} \mathrm{particles} / \mathrm{mL}$ \\
\hline Hydrodynamic diameter & $49.6 \mathrm{~nm}$ \\
\hline Zeta potential & $-30.6 \mathrm{mV}$ \\
\hline pH of the solution & 6.3 \\
\hline Particle surface coating & Polyvinylpyrrolidone (PVP) \\
\hline Solvent & Milli-Q water \\
\hline
\end{tabular}

Anaerobically digested sludge was obtained from the Robert O. Pickard Environmental Center (ROPEC), which is the central wastewater treatment plant in Ottawa, Canada. It receives approximately $390 \mathrm{ML} / \mathrm{d}$ of wastewater (City of Ottawa, 2014) from residential and commercial sources in the region. The sludge for the study was collected from mesophilic anaerobic digesters at ROPEC, the properties of which are provided in Table 6-2.

Table 6-2 Characteristics of anaerobically digested sludge.

\begin{tabular}{|l|l|}
\hline TP (total phosphorus) & $122 \pm 8 \mathrm{mg} / \mathrm{L}$ \\
\hline TN (total nitrogen) & $1043.3 \pm 33 \mathrm{mg} / \mathrm{L}$ \\
\hline TS (total solids) & $17720 \pm 95 \mathrm{mg} / \mathrm{L}$ \\
\hline TVS (total volatile solids) & $9803.3 \pm 45 \mathrm{mg} / \mathrm{L}$ \\
\hline pH & $7.4 \pm 0.1$ \\
\hline
\end{tabular}

Data are the mean of three replicate measurements \pm standard deviation.

Loamy topsoil from Artistic Landscape Designs Ltd Ottawa, Canada, without compost or chemical fertilizer, was used to simulate the land application of biosolids. Soil samples were sieved through a $2 \mathrm{~mm}$ open sieve, air dried, and stored at $4^{\circ} \mathrm{C}$ in the fridge until use. 
Initial soil analyses measured total organic carbon (TOC), total nitrogen (TN), total phosphorus, $\mathrm{pH}$ and cation exchange capacity (CEC), data shown in Table 6-3.

Table 6-3 Characteristics of the soil.

\begin{tabular}{|l|l|}
\hline TOC & $4248 \pm 12 \mathrm{mg} / \mathrm{kg}$ \\
\hline TN & $184 \pm 7 \mathrm{mg} / \mathrm{kg}$ \\
\hline TP & $13 \pm 2 \mathrm{mg} / \mathrm{kg}$ \\
\hline pH & $6.6 \pm 0.7$ \\
\hline CEC & $30.8 \pm 2 \mathrm{meq} / 100 \mathrm{~g}$ \\
\hline
\end{tabular}

Values are the mean of three replicate measurements \pm standard deviation.

\subsubsection{Conditioning chemicals}

Two inorganic conditioners (ferric chloride $\left(\mathrm{FeCl}_{3}\right)$ and alum $\left(\mathrm{Al}_{2}\left(\mathrm{SO}_{4}\right)_{3} \bullet(14-18) \mathrm{H}_{2} \mathrm{O}\right)$ ) and one organic conditioner (polyacrylamide polymer) were assessed to determine their effect on AgNPs behavior during the sludge conditioning process. These chemicals were chosen because they are widely used in wastewater treatment, particularly for sludge conditioning and dewatering. Their concentrations were selected based on preliminary conditioning experiments performed using anaerobically digested sludge to find the optimal dose for each coagulant (Table S-1). Ferric chloride was purchased from Fisher Scientific in powder form, and a stock solution was prepared at a concentration of 40 $\mathrm{mg} / \mathrm{mL}(4 \% \mathrm{w} / \mathrm{v})$. Aluminum sulfate was purchased from Anachemia Canada Inc. in granular form, and a stock solution was prepared at a concentration of $170 \mathrm{mg} / \mathrm{mL}(17 \%)$. The FloPolymer CA 4600 (SNF Canada) used is a dry cationic polyacrylamide polymer with a high charge density and high molecular weight. A stock solution of the polymer was prepared at a concentration of $2 \mathrm{mg} / \mathrm{mL}(0.2 \%)$ by fast mixing for an hour with a magnetic bar mixer, slow mixing for another hour and then resting for an hour before use. 


\subsubsection{Experimental design}

The study was performed in two stages. The first stage investigated the effect of chemical conditioning on the fate and behavior of AgNPs. Chemical conditioning was conducted in two different media; nanopure water and anaerobically digested sludge. Nanopure water was used as a reference media to observe the interaction of AgNPs and chemical conditioners in the absence of other contaminants. There were four reactors for both nanopure water and sludge, one was the control, which had no conditioners and the other three reactors had different chemical conditioners (one reactor for each chemical). Table 6-4 illustrates the properties of each reactor. Each reactor contained $5 \mathrm{~mL}$ of water or sludge. AgNPs were added to each reactor at a concentration of $0.1 \mathrm{mg} / \mathrm{mL}$ and mixed by vortex for $30 \mathrm{~s}$. The conditioning process was conducted by first adding the conditioning chemical, fast mixing for $1 \mathrm{~min}$, and slow mixing for $30 \mathrm{~min}$. The $\mathrm{pH}$ was recorded after the chemical addition and, for ferric chloride and alum, was adjusted to the desired value using $1 \mathrm{M} \mathrm{NaOH}$ (sodium hydroxide). Table 6-4 illustrates the concentrations of the conditioning chemicals dosed and the $\mathrm{pH}$ values in water and sludge. After conditioning, water and sludge samples were prepared for TEM analysis. The concentration of total and dissolved silver in the sludge samples before and after the conditioning process was measured using SP-ICP-MS. Samples were prepared by filtering through a $0.45 \mu$ m nylon filter before the SP-ICP-MS analysis.

The second stage of this study examined the effect of AgNPs on the relative population of selected phyla in soil after sludge application. Five $250 \mathrm{~mL}$ clean glass beakers were each filled with $100 \mathrm{~g}$ soil, and the AgNPs and conditioner concentrations in reactors are

provided in Table 6-5. There were two controls: control 1 received unconditioned 
anaerobically digested sludge that had no AgNPs, while control 2 received the same sludge but was previously mixed with AgNPs. The other three reactors received sludge that was mixed with the same concentration of AgNPs and was subjected to chemical conditioning using a different coagulant for each reactor as described in stage one. A volume of $62 \mathrm{~mL}$ of anaerobically digested sludge with $2 \%$ initial solids concentration was added to each soil reactor. After the addition of sludge, the soil moisture content was $60 \%$ at the start of the experiment. The sludge/soil ratio was $0.01 \mathrm{~g} / \mathrm{g}$ (dry weight), and it was selected based on the biosolids land application guidelines at a rate of 8 ton/ha every 5 years (OME \& OMAFR, 1996). Also, other studies were considered in choosing the sludge application ratio (Colman et al., 2013; Whitley et al., 2013; Johansson et al., 1999). The initial AgNPs concentration in sludge before conditioning was $2 \mathrm{mg} \mathrm{AgNPs/g} \mathrm{sludge} \mathrm{(dry} \mathrm{weight)} \mathrm{that}$ corresponded to $20 \mathrm{mg} \mathrm{AgNPs} / \mathrm{kg}$ soil when sludge was combined with soil. The AgNPs concentration used represents the higher range of the published values of their predicted and recorded concentrations in wastewater sludge (CCME, 2010; EPA, 2018; Blaser et al., 2008; Gottschalk et al., 2013). The reactors were incubated with day/night cycles of 8/16 $\mathrm{h}$ at a constant temperature of $22 \pm 1^{\circ} \mathrm{C}$. This study was carried out for $105 \mathrm{~d}$, during which several chemical and biological analyses were conducted to examine the changes in soil characteristics and microbial activity. Moisture content was maintained at $45 \%$ for all soil reactors by monitoring the soil every three days. The biological analyses carried out were heterotrophic plate count (HPC), bacterial Live/Dead viability staining, and DNA analyses using quantitative polymerase chain reaction (qPCR). The chemical analyses involved cation exchange capacity (CEC), $\mathrm{pH}$, total solids (TS), moisture content (MC), and total organic content (TOC). The concentration of AgNPs in sludge during the first stage was 
higher from the second stage to be able to detect and observed the AgNPs by TEM, and it was used by previous studies as a very acute dose of AgNPs (Liang et al., 2010; Sun et al., 2013).

Table 6-4 Concentrations of conditioners and final $\mathrm{pH}$ values in water and sludge reactors.

\begin{tabular}{|c|c|c|c|}
\hline \multirow{2}{*}{$\begin{array}{c}\text { Water/sludge } \\
\text { reactors }\end{array}$} & $\begin{array}{c}\text { Conditioners } \\
\text { concentrations in } \\
\text { sludge (mg/mg) }\end{array}$ & \multicolumn{2}{|c|}{$\mathbf{p H}( \pm \mathbf{0 . 2})$} \\
\cline { 3 - 4 } & 0 & Water & Sludge \\
\hline Control & 0.1 & 7.0 & 7.6 \\
\hline Ferric chloride & 0.7 & 7.3 & 7.2 \\
\hline Alum & 0.007 & 7.3 & 7.1 \\
\hline Polymer & \multicolumn{2}{|c}{} &
\end{tabular}

Table 6-5 Concentrations of AgNPs and conditioners in soil reactors.

\begin{tabular}{|l|c|c|c|}
\hline \multicolumn{1}{|c|}{ Soil reactors } & $\begin{array}{c}\text { AgNPs/TS of } \\
\text { sludge (mg/g) }\end{array}$ & $\begin{array}{c}\text { AgNPs/soil } \\
\text { (mg/kg) }\end{array}$ & $\begin{array}{c}\text { Conditioner } \\
\text { concentration in sludge } \\
(\mathbf{m g} / \mathbf{m g})\end{array}$ \\
\cline { 1 - 1 } Control 1 & No Ag np. & No Ag np. & 0 \\
\hline Control 2 & & & 0 \\
\cline { 1 - 1 } Ferric chloride & \multirow{2}{*}{2} & \multirow{2}{*}{20} & 0.1 \\
\cline { 1 - 1 } Alum & & & 0.7 \\
\cline { 1 - 1 } Polymer & & & 0.007 \\
\hline
\end{tabular}

\subsubsection{TEM and EDS}

Particle size, shape, and morphology were examined using TEM technique. The samples were prepared in the lab by placing approximately $5 \mu \mathrm{L}$ of conditioned sludge on $3.05 \mu \mathrm{m}$ supported copper grid film coated with formvar/carbon, with 200 mesh and a hole size of approximately $97 \mathrm{~nm}$ from Ted Pella Inc. There were three replicates for each sample. After preparation, the samples were taken to the Nano Imaging facility at Carleton University for TEM analysis, which was done using FEI Tecnai G2 F20 (Netherlands). In addition to morphological analyses, the elemental composition of the samples was analyzed twice, using energy-dispersive x-ray spectroscopy (EDS) coupled to TEM. This 
combination allows EDS to provide an elemental composition of an area that is only a few nanometers in diameter. The results are shown as a plot of the emitted x-rays versus their energy in $\mathrm{keV}$. The plot has many peaks, each corresponds to specific elements.

\subsubsection{ICP-MS and SP-ICP-MS Analysis}

All ICP-MS analyses were conducted using a PerkinElmer NexION 300D ICP-MS system in three replicates. Total $\mathrm{Ag}$ in solutions/suspensions was determined on samples acidified to $1 \% \mathrm{HNO}_{3}$ (TraceMetal grade, Fisher Chemical) at least $24 \mathrm{~h}$ prior to analysis, using the ICP-MS in standard mode. SP-ICP-MS analysis was performed using the same ICP-MS but operating in single-particle mode using the Syngistix Nano-Application module (PerkinElmer, Inc., Waltham, MA). Other instrumental and analytical details are available in Schwertfeger et al. (2017).

\subsubsection{Chemical analysis}

The chemical analysis included the initial analysis of the soil and sludge samples, as well as the analyses carried out every $15 \mathrm{~d}$ from the start of the study to its completion. All chemical analyses were replicated three times and performed according to Standard Methods (APHA et al., 2017). The soil was analyzed for CEC at the beginning of the experiment using a standard method employed by the Natural Resources Conservation Service (Rebecca, 2004). The analysis methodology involves using $1 \mathrm{M}$ ammonium acetate $\left(\mathrm{NH}_{4} \mathrm{OAc}\right)$ at $\mathrm{pH} 7$ (neutral $\left.\mathrm{NH}_{4} \mathrm{OAc}\right)$. Soil samples were prepared for $\mathrm{pH}$ analysis by suspending $5 \mathrm{~g}$ of soil in $5 \mathrm{~mL}$ of water ( $1 \mathrm{~g}: 1 \mathrm{~mL}$ ratio), vortexing the suspension for $30 \mathrm{~s}$, then taking the $\mathrm{pH}$ measurement using an Orion-Thermo Orion Star тм A326 pH/DO portable meter. The percentages of TS and MC were determined by drying $5 \mathrm{~g}$ of the soil 
sample in an oven at $105^{\circ} \mathrm{C}$ for $24 \mathrm{~h}$. The $\mathrm{TS} \%$ is the sample weight after drying divided by sample weight before drying and multiplied by 100 , while the $\mathrm{MC} \%$ is calculated by taking the difference in the sample weight before and after drying, dividing it by the weight of the dried sample and multiplying by $100 \%$. MC was maintained at $45 \%$ for all five soil reactors by taking three measurements every 2 to $3 \mathrm{~d}$. The soil reactors were also analyzed for TOC, using TOC- $\mathrm{V}_{\mathrm{CPH} / \mathrm{CPN}}$ and TOC-control V software. For each TOC measurement run, one blank and one set of the standard solution concentrations were used to construct a calibration curve, and three replicates were analyzed. Soil samples were prepared by suspending $1 \mathrm{~g}$ of soil in $10 \mathrm{~mL}$ of $\mathrm{CaCl}_{2}$ solution $(1: 10 \mathrm{w} / \mathrm{v}, 0.01 \mathrm{M})$. The suspensions were put in a shaker for two hours and centrifuged at $1,800 \times \mathrm{g}$ for $12 \mathrm{~min}$. The supernatant was then taken for the TOC analysis.

\subsubsection{Microbiological analyses}

\subsubsection{Heterotrophic plate count}

Changes in the viability of heterotrophic bacteria in the soil samples were investigated every 15 days using the spread plate method. Tryptic soy agar (TSA) was used as the growth medium. The TSA was prepared by adding $30 \mathrm{~g}$ of the powdered TSA to $1 \mathrm{~L}$ distilled water and dissolved by boiling. After the medium had cooled to approximately $50^{\circ} \mathrm{C}$, a volume of $22 \mathrm{~mL}$ was poured into each Petri plate and stored at $4^{\circ} \mathrm{C}$ for a maximum of one week. Soil samples were prepared by suspending $0.1 \mathrm{~g}$ of the soil samples in $0.9 \mathrm{~mL}$ phosphate buffered saline (PBS) (with $0.1 \%$ Tween 80 ) and vortexed for $30 \mathrm{~s}$. Serial dilutions were made, and three $0.1 \mathrm{~mL}$ replicates of 10E-04, 10E-05 and 10E-06 dilutions 
were inoculated onto the TSA plates and incubated in the dark at $34 \pm 1^{\circ} \mathrm{C}$. Colony forming units (CFU) were calculated after $24 \mathrm{~h}$ of incubation.

\subsubsection{Live/Dead bacterial viability staining assay}

The distribution of Live/Dead bacterial cells in the soil reactors was investigated by staining with fluorescent dyes and observing with the Nikon Eclipse Ti-E Microscope. The soil samples were stained with a BacLight staining reagent kit (Thermo Fisher Scientific) that was prepared by dissolving SYTO 9 stain, and propidium iodide stain in $5 \mathrm{~mL}$ of sterilized deionized water. The soil samples were prepared for Live/Dead analysis by suspending $0.1 \mathrm{~g}$ of soil in $0.9 \mathrm{~mL}$ of PBS solution with $0.1 \%$ Tween 80 . The suspension was vortexed for $30 \mathrm{~s}$ at high speed then left to settle for $30 \mathrm{~min}$. A known volume of the supernatant was mixed with an equal amount lof the staining reagent, and the mixture was incubated in the dark for 15 min, after which $17 \mu \mathrm{L}$ of the mixture was placed between a slide and a $22 \mathrm{~mm}$ square coverslip. The samples were then ready for observation under the fluorescence microscope, where the ratio of Live/Dead bacterial cells could be

quantified. Three tests were done for each soil sample, and three random fields of views (FOV) were chosen on each slide where images were taken. Three images were taken at each FOV using 3 UV filters, GFP-1, Cy-3 and GFPHQ to capture live, dead and a combination of live and dead microbial cells respectively. The three sets of images were assessed, and the most representative was chosen to report, and the live cell percentage was estimated by dividing the number of live cells by the total number of cells in the image. 


\subsubsection{Bacterial population analysis}

\subsection{Bacterial phyla}

The five most prevalent bacterial phyla found in soil and sludge (Acidobacteria, Actinobacteria, Bacteroidetes, Firmicutes, and Proteobacteria) were selected for investigation. Each soil reactor was analyzed for the presence and relative population of each phylum every $15 \mathrm{~d}$ over the 105 days experiment time frame. Three replicate DNA extractions were done for each soil reactor using the PowerSoil DNA Isolation Kit from MO BIO Laboratories Inc. (QIAGEN Online Shop), and the concentration of extracted DNA was measured using the Qubit ${ }^{\circledR}$ 2.0 Fluorometer with the dsDNA HS Assay Kits (Molecular Probes, Life technology). The extracted DNA from the samples were then used as template DNA for the phylum-specific qPCR protocols. Each phylum was amplified individually using phylum-specific qPCR primer sets.

\subsection{Primers}

Primers were purchased from Life Technologies in Burlington, Ontario, and they were selected based on some of the latest published studies. Primer references and properties are shown in Table 6-6. 
Table 6-6 Phyla primer pairs used in qPCR assay.

\begin{tabular}{|c|c|c|c|c|c|}
\hline Phylum & $\begin{array}{l}\text { Amplicon } \\
\text { size (bp) }\end{array}$ & $\begin{array}{l}\text { Primer } \\
\text { name }\end{array}$ & Primer sequence $\left(5^{\prime}->3^{\prime}\right)$ & $\begin{array}{l}\text { Annealing } \\
\text { temperature } \\
\left({ }^{\circ} \mathrm{C}\right) \\
\end{array}$ & References \\
\hline \multirow{2}{*}{ Acidobacteria } & \multirow{2}{*}{500} & fAcid31 & GATCCTGGCTCAGAATC & \multirow{2}{*}{55.9} & \multirow{2}{*}{$\begin{array}{l}\text { (Fierer et al., } \\
\text { 2005) }\end{array}$} \\
\hline & & rEub518 & ATTACCGCGGCTGCTGG & & \\
\hline \multirow{2}{*}{ Actinobacteria } & \multirow{2}{*}{166} & fActi & GRDACYGCCGGGGTYAACT & \multirow{2}{*}{57.2} & \multirow{2}{*}{$\begin{array}{l}\text { (Pfeiffer et al., } \\
\text { 2014) }\end{array}$} \\
\hline & & rActi & TCWGCGATTACTAGCGAC & & \\
\hline \multirow{2}{*}{ Bacteroidetes } & \multirow{2}{*}{181} & fBdet & GCACGGGTGMGTAACRCGTACCCT & \multirow{2}{*}{61} & \multirow{2}{*}{$\begin{array}{l}\text { (Pfeiffer et al., } \\
\text { 2014) }\end{array}$} \\
\hline & & rBdet & GTRTCTCAGTDCCARTGTGGG & & \\
\hline \multirow{2}{*}{ Firmicutes } & \multirow{2}{*}{156} & fFirm & CAGCAGTAGGGAATCTTC & \multirow{2}{*}{55.3} & \multirow{2}{*}{$\begin{array}{l}\text { (Pfeiffer et al. } \\
\text { 2014) }\end{array}$} \\
\hline & & rFirm & ACCTACGTATTACCGCGG & & \\
\hline \multirow{2}{*}{ Proteobacteria } & \multirow{2}{*}{140} & 767 fProt & AAGCGTGGGGAGCAAACA & \multirow{2}{*}{54.8} & \multirow{2}{*}{$\begin{array}{l}\text { (Morales \& } \\
\text { Holben, 2009) }\end{array}$} \\
\hline & & 907rProt & CCGTCAATTCMTTTRAGTTT & & \\
\hline \multirow{2}{*}{$\begin{array}{l}16 \mathrm{~S} \\
\text { (any bacteria) }\end{array}$} & \multirow{2}{*}{180} & $338 \mathrm{~F}$ & ACT CCT ACG GGA GGC AGC AG & \multirow{2}{*}{61.9} & \multirow{2}{*}{$\begin{array}{l}\text { (Shakya et al., } \\
\text { 2013) }\end{array}$} \\
\hline & & $518 \mathrm{R}$ & ATT ACC GCG GCT GCT GG & & \\
\hline
\end{tabular}

\subsubsection{3 qPCR protocols and annealing temperature optimization}

Phylum analysis was done using individual qPCR protocol for each soil mixture every 15 d over the 105 days experiment period. In each qPCR run, there were three replicate qPCR reactions for each genomic DNA sample, and three controls for all phyla assessed. The reactions were performed using the CFX96 Touch Real-Time PCR Detection System (BioRad) and analyzed with the CFX Manager Software. The qPCR protocols were the same for all phyla, except for the annealing temperatures. The qPCR protocol consisted of an initial denaturation step at $95^{\circ} \mathrm{C}$ for $5 \mathrm{~min}$, followed by 40 cycles at $95^{\circ} \mathrm{C}$ for $10 \mathrm{~s}$, annealing for $20 \mathrm{~s}$ (Table 6-6), an extension at $72^{\circ} \mathrm{C}$ for $15 \mathrm{~s}$ and, finally, a melt curve analysis from 65 to $95^{\circ} \mathrm{C}$ with a $0.5^{\circ} \mathrm{C}$ increase every $5 \mathrm{~s}$. Every qPCR reaction consisted of $12.5 \mu \mathrm{L}$ qPCR buffer, $0.5 \mu \mathrm{L}(200 \mathrm{nM})$ forward primer, $0.5 \mu \mathrm{L}(200 \mathrm{nM})$ reverse primer, DNA template equilibrated to $30 \mathrm{ng}$ concentration, and water to $25 \mu \mathrm{L}$ as the final reaction volume. The qPCR reaction/buffer solution was the SsoFast EvaGreen Supermix purchased from Bio-Rad Laboratories Ltd, Canada, which contains dNTPs, Sso7d fusion 
polymerase, $\mathrm{MgCl}_{2}$, EvaGreen dye, and stabilizers. Preliminary experiments were done to optimize the annealing temperature for each phylum protocol, and the annealing temperature that maximized the primer amplification was determined using the extracted soil DNA as a template in a thermal gradient qPCR reaction, with a different annealing temperature range for each phylum.

\subsubsection{4 qPCR results analysis}

Universal 16S ribosomal RNA primers were used to amplify an approximately $180 \mathrm{bp}$ sequence, which was used to calculate the baseline $16 \mathrm{~S}$ gene copy number and compare it with each of the five phyla specific calculated gene copy numbers. This data generated relative population trends for each phylum over time. The data analysis was based on the following fundamental assumptions:

1. Cq is equivalent to $3.33 \log _{10}$ (target amplicon concentration).

Many studies have used a control curve to relate the number (concentration) of DNA in the qPCR product to the corresponding Cq. Svec et al. (2015) stated that constructing a control curve with $\log _{10}$ of DNA number versus the Cq that resulted from the qPCR run of serial concentrations of DNA, will be linear with a slope of 3.33 when the qPCR efficiency is $100 \%$ (Svec et al., 2015).

2. Every bacterium has $1 \sim$ fg of DNA.

The concentration of the DNA template used in the qPCR reaction was $30 \mathrm{ng}$. The qPCR analysis in this study relies on a relative estimate of the bacterial population. If it is assumed that every bacterium has $1 \sim$ fg of DNA, then $30 \mathrm{ng}$ of DNA is equal to $3 \mathrm{X} 10 \mathrm{E}+07$ bacteria. This is a relative estimate because the soil extracted DNA is present in many living things other than bacteria, such as plants and eukaryotes. 
3. Every phylum has a specific copy number of $16 \mathrm{~S}$ rRNA.

Based on a recent study by (Větrovský \& Baldrian, 2013), each phylum has a specific copy number of $16 \mathrm{~S}$ rRNA, as shown in Table 6-7.

Table 6-7 Copy number of 16S rRNA in each bacterial genome (Větrovský \& Baldrian, 2013).

\begin{tabular}{|c|c|c|}
\hline Phylum & 16S rRNA/genome & Mean 16S rRNA/genome \\
\hline Acidobacteria & 1 & 1 \\
\hline Actinobacteria & $3.3 \pm 1.7$ & 3.3 \\
\hline Bacteroidetes & $3.5 \pm 1.5$ & 3.5 \\
\hline Firmicutes & $5.8 \pm 2.8$ & 5.8 \\
\hline Proteobacteria & $3.96 \pm 1.7$ & 4.0 \\
\hline
\end{tabular}

\subsubsection{Statistical analyses}

Most results are reported with the average of three replicates \pm standard deviation. Comparisons and significant differences between different soil reactors over time (control 1 versus control 2 and control 2 versus other soil reactors) were performed using a oneway analysis of variation (ANOVA) at a 95\% confidence level. Significant differences are those with a p-value $<0.05$. If the ANOVA-test indicated a significant difference, a T-test was performed between each of the two data sets. In addition, the coefficient of variance (CV) was estimated for each data set, and significant differences are those with a CV > 0.05 (i.e., the level of confidence $\geq 95 \%$ ). 


\subsection{Results and discussion}

\subsubsection{Effects of sludge on AgNPs}

AgNPs did not show any chemical or morphological change when suspended in water. TEM imaging showed that AgNPs in water continue to be dispersed with no effects, as shown in Figure 6-1(a). While in sludge, AgNPs did have some chemical and morphological changes just after they were added to the wastewater sludge. From TEM observations in Figure 6-1(b), AgNPs appear to be modified and affected by the presence of the sludge constituents. Some of the changes that can be observed are surface coating, dissolution, and agglomeration. Also, Figure 6-1 shows the EDS analysis results, which display the elemental composition of each water and sludge samples. Most of these peaks are X-rays given off as electrons return to the specific elements found in the tested sample. Figure 6-1 shows the presence of carbon $(\mathrm{C})$, copper $(\mathrm{Cu})$, and silver $(\mathrm{Ag})$, with carbon having highest peaks indicating high concentration, which is due to the carbon presented in the support copper grid film (copper grid film is coated with formvar/carbon). In sludge, the EDS spectrum showed several peaks representing different elements found in the tested sludge.

TEM images showed that sludge caused chemical and morphological alterations in AgNPs. The changes are likely due to the interactions between AgNPs and sludge constituents, including oxidizers, sulfur compounds, chloride compounds, and organic matter. For example, the presence of oxidizers in sludge (e.g., $\mathrm{O}_{2}$ and $\mathrm{H}_{2} \mathrm{O}_{2}$ ) can lead to the oxidative

dissolution of AgNPs, in which, the nanoparticle surface is oxidized to $\mathrm{Ag}_{2} \mathrm{O}$ which forms a layer on the nanoparticle surface (Zhang et al., 2018). The oxidative dissolution of AgNPs 
can cause an increase in the nanoparticle size after dissolution with no morphology change, which other studies (Peretyazhko et al., 2014) attributed to the Ostwald ripening mechanism, in which the nanoparticles dissolve and redeposit as larger nanoparticles. Sulfidation of AgNPs can also occur and result in several morphological changes, including forming a shell of $\mathrm{Ag}_{2} \mathrm{~S}$ NPs around the AgNPs surface until all available AgNPs are converted to $\mathrm{Ag}_{2} \mathrm{~S}$ NPs (Thalmann et al., 2016), or formation of a nanobridge of $\mathrm{Ag}_{2} \mathrm{~S}$ NPs that connects AgNPs with each other (Levard et al., 2011). Chlorination of AgNPs was also reported in previous studies, and like sulfidation, can form a shell of $\mathrm{AgCl}$ around the AgNPs surface, which can prevent the dissolution of AgNPs (Li et al., 2010). In addition, the presence of organic matter results in the surface coating of AgNPs, which can stabilize the particles and maintain their dispersive state in the sludge, and thus affect the behavior and state of AgNPs. Cumberland \& Lead (2009) showed that approximately $1 \%$ of the AgNPs dissolved after AgNPs (average size 13-15 nm) were added to a solution containing humic substances and sodium or calcium to mimic environmentally relevant conditions. Another study by Tseng et al. (2009) showed that silver ions are not only released from silver salts but metallic silver as well if conditions are suitable. 

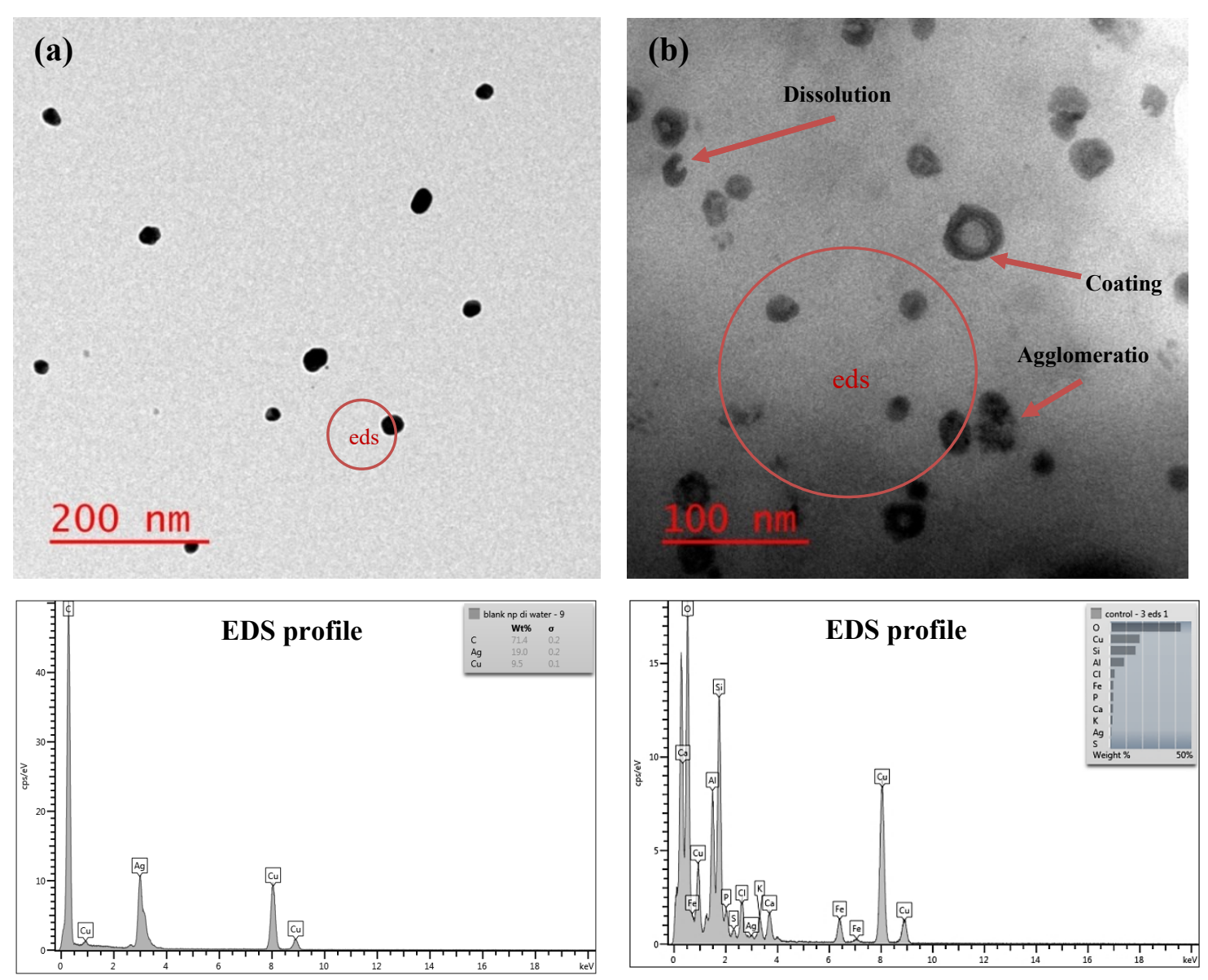

Figure 6-1 TEM image with its EDS profile (elemental composition) of AgNPs suspended in water (a) and sludge (b).

\subsubsection{Effect of chemical conditioning on AgNPs}

After spiking with AgNPs, the coagulation and flocculation were performed for each water and sludge reactor using three chemicals: ferric chloride, alum, and polymer. From visual observation, after $30 \mathrm{~min}$ of slow mixing, the coagulation caused the formation of larger, less mobile particle clusters. SP-ICP-MS analysis was performed to examine the concentration of AgNPs before and after the conditioning process. ICP-MS and SP-ICPMS were used to measure the total and particulate silver (AgNPs) concentrations in the water and sludge reactors and dissolved silver concentrations were calculated from these 
measurements. Table 6-8 presents the dissolved and nanoparticle silver concentrations for both water and sludge with and without conditioners. The controls had no conditioners and all the reactors were spiked with $100 \mathrm{mg} \mathrm{AgNPs} / \mathrm{L}$. The total silver measured were 48.5 $\mathrm{mg} / \mathrm{L}$ and $53 \mathrm{mg} / \mathrm{L}$ for the water and sludge controls, respectively, and of these 33 and $26 \%$ are AgNPs in water and sludge, respectively. This indicates that approximately half of the spiked silver was lost during sample pre-treatment and filtration steps prior to ICP-MS and SP-ICP-MS analysis, and similar findings were reported by other studies (Schwertfeger et al., 2017). The loss was similar in nanopure water and sludge samples indicating that the loss could not be due to chemical reactions with sludge constituents. In addition, the slightly higher percentage of silver recovery in sludge (53\%) rather than water (48.5\%) indicates that some of the AgNPs was likely dissolved in sludge due to sludge characteristics and reactions with sludge constituents allowing a higher amount of silver to pass through the filter.

After the conditioning and aggregation of solids, there were small quantities of AgNPs (less than $0.39 \%$ of total silver in water reactors and $0.06 \%$ in sludge reactors) remaining in the liquid phase. This confirms that the conditioning chemicals were very successful in removing AgNPs from the aqueous phase by flocculation. The concentration of silver detected in all reactors after the conditioning process was mostly in dissolved form (Table 6-8). Only 33 and $26 \%$ of 48.5 and $53 \mathrm{mg} / \mathrm{L}$ total silver were still in the nanoparticle form in the control water and sludge samples indicating that a substantial portion of AgNPs was dissolved shortly after spiking. The silver concentrations in samples conditioned with alum could not be measured due to the interference of aluminum ions with the analytical method and the instrument. 
Table 6-8 Total, particulate, and dissolved silver concentrations in conditioned and control nanopure water and sludge samples measured by SP-ICP-MS and ICP-MS analysis.

\begin{tabular}{|c|c|c|c|c|c|}
\hline \multicolumn{2}{|c|}{ Reactor } & \multirow{2}{*}{$\begin{array}{c}\begin{array}{c}\text { Spiked AgNPs } \\
\text { concentration } \\
(\mathrm{mg} / \mathrm{L})\end{array} \\
100.0 \\
\end{array}$} & \multirow{2}{*}{$\begin{array}{c}\begin{array}{c}\text { Measured } \\
\text { total Ag } \\
\text { concentration } \\
(\mathbf{m g} / \mathrm{L})\end{array} \\
48.5\end{array}$} & \multirow{2}{*}{$\begin{array}{c}\begin{array}{c}\text { Particulate Mass* } \\
(\%)\end{array} \\
33\end{array}$} & \multirow{2}{*}{$\begin{array}{c}\begin{array}{c}\text { Dissolved Mass** } \\
(\%)\end{array} \\
67\end{array}$} \\
\hline \multirow{4}{*}{ Water } & Control & & & & \\
\hline & $\mathrm{FeCl}_{3}$ & 100.0 & 0.3 & 3 & 97 \\
\hline & Alum & 100.0 & 0.4 & NA & NA \\
\hline & Polymer & 100.0 & $<0.003$ & 1 & 99 \\
\hline \multirow{4}{*}{ Sludge } & Control & 100.0 & 53.0 & 26 & 74 \\
\hline & $\mathrm{FeCl}_{3}$ & 100.0 & 0.1 & 12 & 88 \\
\hline & Alum & 100.0 & $<0.003$ & NA & NA \\
\hline & Polymer & 100.0 & $<0.003$ & 20 & 80 \\
\hline
\end{tabular}

\subsubsection{Structural analyses of AgNPs under different conditioning chemicals}

All conditioning chemicals were very effective in removing AgNPs via attachment and aggregation.

\section{Ferric chloride and aluminum sulfate}

The performance of ferric chloride and aluminum sulfate treatments were similar, and both resulted in the incorporation of AgNPs into flocs, as seen in Figures 6-2 and 6-3, and the formation and structure of the flocs were different. Ferric chloride and aluminum sulfate dissociate in water and produce metallic ions $\mathrm{Fe}^{3+}$ and $\mathrm{Al}^{3+}$, which in turn react with water molecules to create hydrated reaction products such as $\mathrm{Fe}\left(\mathrm{H}_{2} \mathrm{O}\right)_{6}{ }^{3+}$ and $\mathrm{Al}\left(\mathrm{H}_{2} \mathrm{O}\right)_{6}{ }^{3+}$. The hydrolysis process can also result in the formation of ferric and aluminum hydroxide $\left(\mathrm{Fe}(\mathrm{OH})_{3}\right.$ and $\left.\mathrm{Al}(\mathrm{OH})_{3}\right)$, which can further dissociate into ionic compounds. The ions and ionic compounds are adsorbed and deposited on particle surfaces, including AgNPs, which leads to the agglomeration of AgNPs in ferric and alum complexes. The performance of 
ferric chloride and aluminum sulfate, and the entrapment of AgNPs in ferric and aluminum flocs were evident when the AgNPs were suspended in nanopure water. However, the behavior of AgNPs in the presence of ferric and aluminum was more complex in sludge than in water, due to the presence of organic matter and many other constituents. 


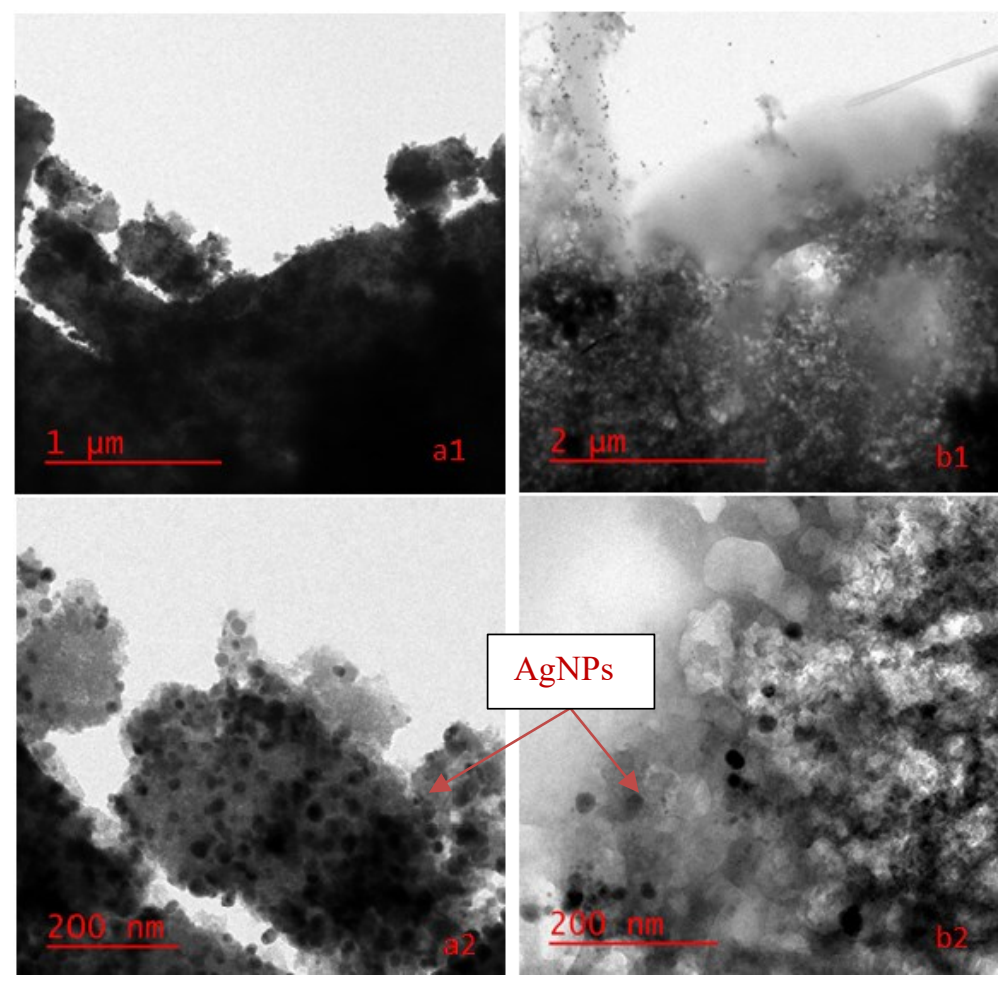

Figure 6-2 TEM images show ferric chloride treatment effect on AgNPs suspended in water (a) and sludge (b), 1 and 2 refer to different scales.

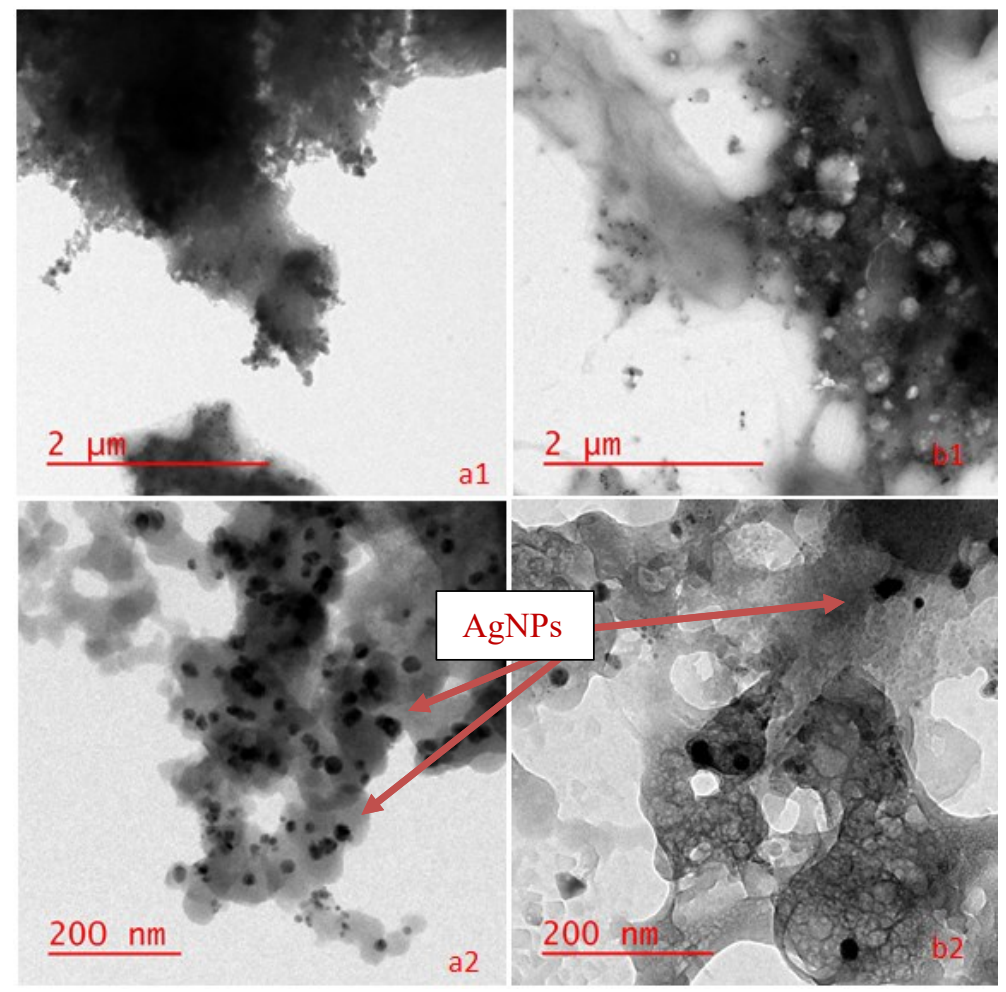

Figure 6-3 TEM images show alum treatment effect on AgNPs suspended in water (a) and sludge (b), 1 and 2 refer to different scales. 


\section{Synthetic polymer}

Unlike inorganic conditioners such as ferric and aluminum salts, synthetic polymers can effectively achieve flocculation without forming chemical precipitates and flocs. The addition of polymer resulted in the coating and aggregation of AgNPs, as illustrated in Figure 6-4(a). In sludge, the clarity of images was not as good due to the flocculation of sludge particles and organic matter, as well as some dissolution of AgNPs, as shown in Figure 6-4(b). However, the results of SP-ICP-MS highlighted the superb capability of polymer to remove AgNPs from the aqueous phase of sludge, as the final total silver concentration after polymer conditioning was $<0.003 \mathrm{mg} / \mathrm{L}$ in both water and sludge. Polymer affects the surface chemistry and charge of AgNPs and impacts their status and transformation. Though this also applies to other coagulant chemicals, polymers have a much higher molecular weight, charge and thus stronger influence.

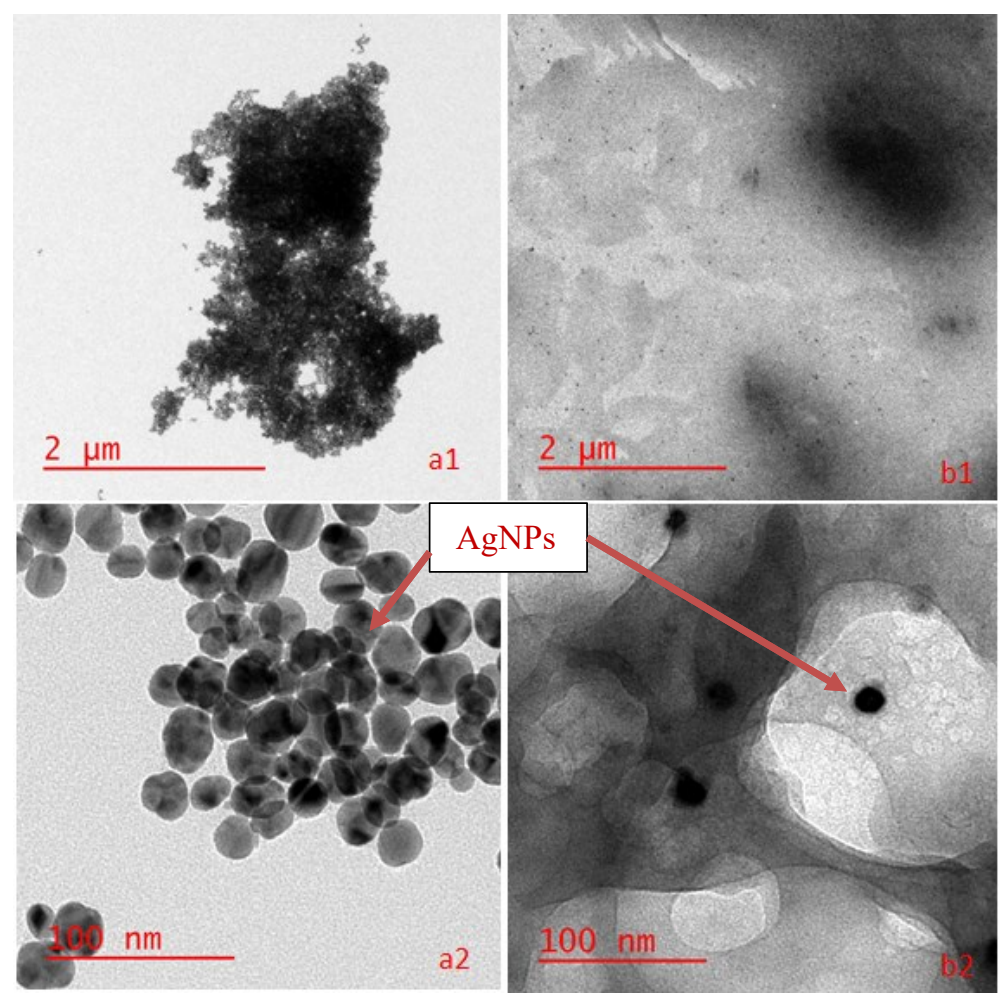

Figure 6-4 TEM images show polymer treatment effect on AgNPs suspended in water (a) and sludge (b), 1 and 2 refer to different scales. 


\subsubsection{Impact of AgNPs after land application of biosolids}

The next phase of the study investigated the effect of AgNPs treated with organic and inorganic conditioners on soil microorganisms by simulating the land application of biosolids using soil reactors.

\subsubsection{TOC and $\mathrm{pH}$}

The TOC of the soil decreased on the day sludge was applied to soil (day 0 ) in the ferric chloride and alum reactors, however, the TOC of the other reactors also decreased over time and they reached a similar level with the ferric chloride and alum reactors at the end of the experiment, as seen in Figure 6-5(a). There were no significant differences between the controls and other three soil reactors over time, and based on the T-test analysis, pvalues were between 0.4 to 0.9 . The TOC reduction was high during the first month (ranging from $15 \%$ to $42 \%$ ) compared with the following months (day 30 to 105 ) where the decrease in TOC was insignificant. Also, as seen in Figure 6-5, there was an increase in the concentrations of TOC between days 45 and 60, particularly in the polymer reactor which could be due to analysis errors. Soil $\mathrm{pH}$ is considered to play an important role in the soil microbial population and diversity. There was one unit decrease in $\mathrm{pH}$ from day 0 to day 15 in most reactors, as shown in Figure 6-5(b). This decrease is considered normal as a result of the acclimation of chemical reactions and microorganisms to the new environment. After day 15, $\mathrm{pH}$ readings were stable over the remaining 90 days. Overall, the $\mathrm{pH}$ dropped for all reactors from neutral (a $\mathrm{pH}$ of approximately 7 ) at the beginning of the experiment, to slightly acidic at the end. In general, $\mathrm{pH}$ levels were similar in all soil reactors over time. As a result, the presence of AgNPs did not have significant impact on 
soil TOC and $\mathrm{pH}$ ( $\mathrm{p}$-value $>0.05$ ), which confirms recent findings that also showed no effects of AgNPs and other nanoparticles on soil pH and TOC (Carbone et al., 2014; BenMoshe et al., 2013; Hänsch \& Emmerling, 2010). There are many factors that could potentially affect the TOC level in soil, including $\mathrm{pH}$, temperature, soil aeration, and microbial population. The observed decrease in TOC could be due to microorganism's respiration or decomposition and consumption of organic matter by microorganisms, which compete to survive in a limited carbon environment (no additional carbon was added to the soil reactors during the experiment). In addition, changes in microbial population and activity occur over time. Soil $\mathrm{pH}$ is one of the parameters that have a major influence on the availability of nutrients in the soil, such as nitrogen and phosphorus. At a near neutral $\mathrm{pH}(\mathrm{pH}=7)$, soil nitrogen is available in the form of nitrate $\left(\mathrm{NO}_{3}^{-}\right)$that can be easily taken up by plants due to the rapid conversion of ammonium $\left(\mathrm{NH}_{4}^{+}\right)$to nitrate. With acidic $\mathrm{pH}$, the nitrification process is slow, and only ammonium is available as a source of nitrogen for plants. In alkaline soil, phosphorus tends to form stable minerals which are very difficult to solubilize, which is why it is limited in soil under alkaline conditions.
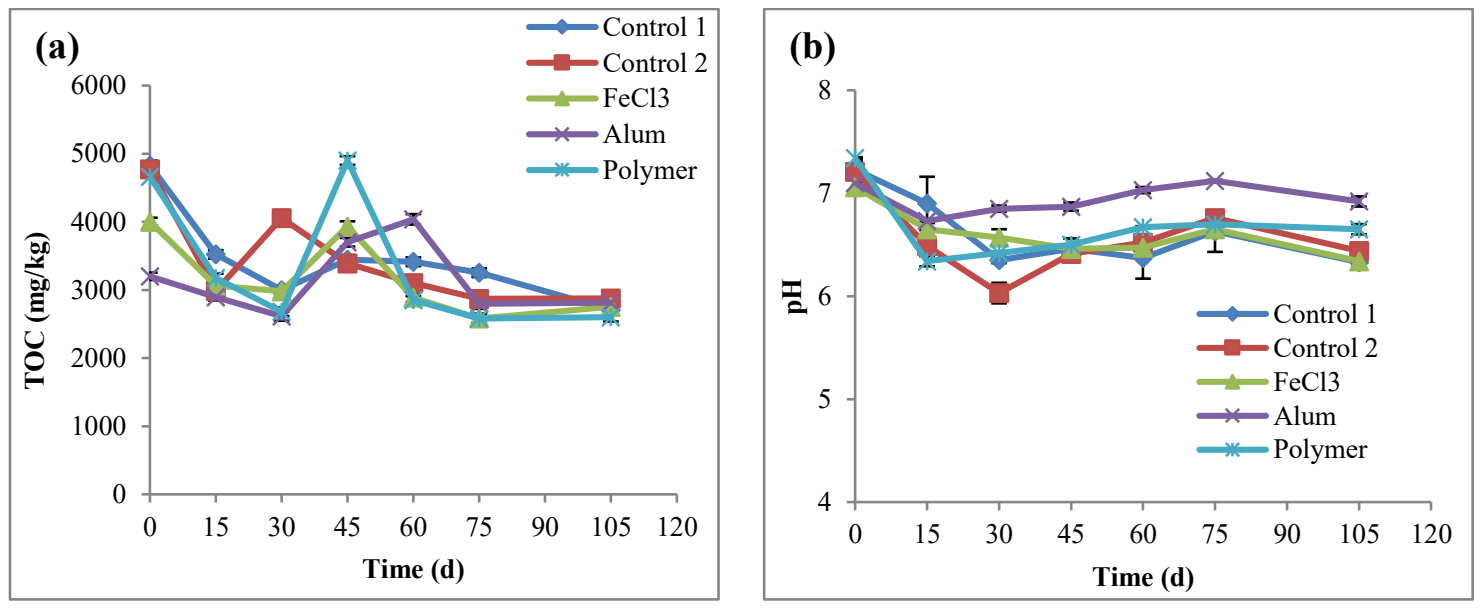

Figure 6-5 Soil TOC concentration (a) and pH (b) over time. 


\subsubsection{Impact of AgNPs on heterotrophic bacteria and Live/Dead viability}

The results showed that on day 0, HPC was similar in all the reactors except alum, which was half a $\log _{10}$ lower, as seen in Figure 6-6(a). T-test analysis showed no significant difference between controls 1 and 2 (p-value was 0.58 ) over time, meaning the presence of AgNPs at this concentration had no significant effect on the heterotrophic bacteria count in the soil. However, the type of chemicals used in sludge conditioning affected the soil HPC trends over time. However, this effect was not significant and compared to control 2 , the $\mathrm{p}$-values were $\approx 0.4,0.14$, and 0.84 for ferric chloride, alum, and polymer soil reactors, respectively. Heterotrophic bacteria use organic compounds as carbon and energy sources (Metcalf \& Eddy, 2003). After land application of biosolids, the results indicate that the alum soil reactor had lower $\mathrm{CFU}$ at day 0 , likely due to the toxicity of alum on soil microorganisms. However, the difference in HPC observed was not significant, and any toxicity was not sustained as the alum soil reactor had the highest CFU at the end of the experiment. Indeed, the results showed no significant differences in CFU between all the soil reactors, which suggests that AgNPs had no significant impact on heterotrophic bacteria in the soil reactors. This is confirmed by previous studies that showed no significant impact of AgNPs when applied on the soil at $10 \mathrm{mg} / \mathrm{kg}$ soil; however, AgNPs at $100 \mathrm{mg} / \mathrm{kg}$ soil severely impacted the HPC in the soil (Carbone et al., 2014). Many factors can contribute to that, including the AgNPs concentration used and the sludge treatment before land application.

The effects of AgNPs on soil microorganisms were also investigated through Live/Dead bacterial viability assay. On day 0 , the percentage of live cells was similar in all the 
reactors. Fluctuations were observed over time, but ANOVA analysis showed no significant differences among the reactors. The percentage of live cells remained constant over time in most reactors, as shown in Figure 6-6(b). As Live/Dead staining is a general representation of the overall bacterial viability and does not assess the bacterial communities in the soil, the fluctuations may be due to specific genera or species adapting and thriving to the experimental conditions. Bacterial Live/Dead staining images are presented in the supplementary data (Figures S-2 to S-6).
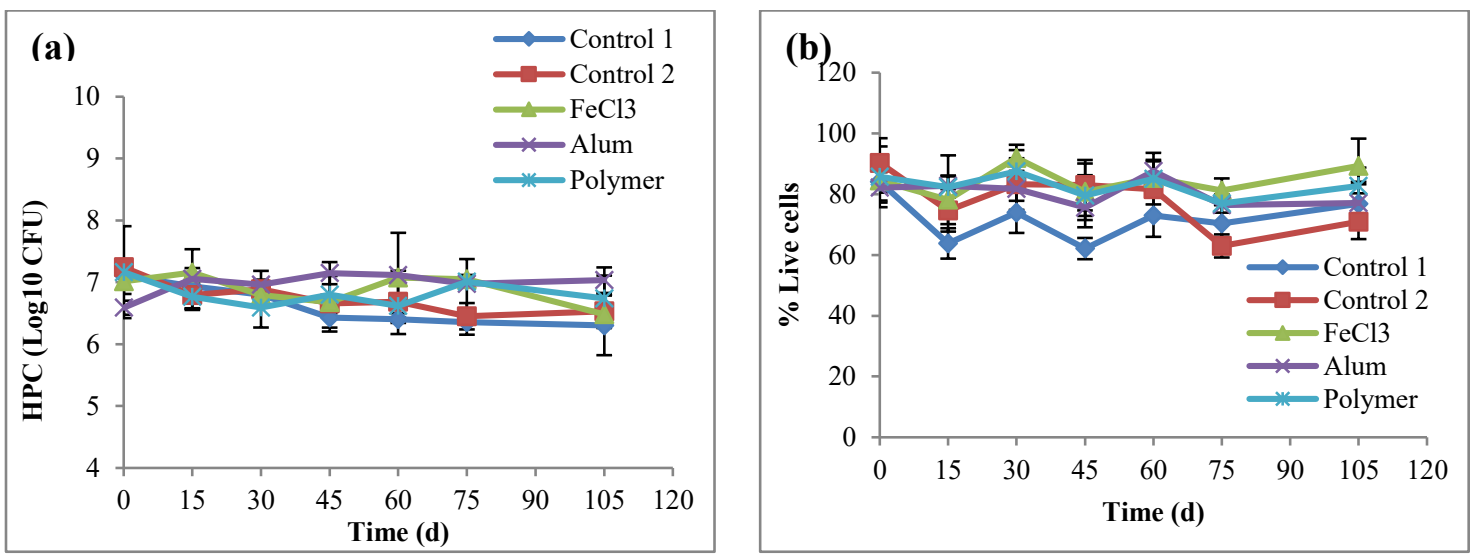

Figure 6-6 HPC, represented by CFU (a) and the percentage of live bacterial cells (b) in soil reactors over time.

\subsubsection{Impact of AgNPs on selected soil microorganisms}

The soil genomic DNA extractions (Figure S-1) were analyzed for the presence of five selected phyla using qPCR. The results were used to determine the average abundance of phyla in all reactors, as shown in Figure 6-7. Overall, the results showed that Acidobacteria was the most abundant phylum among the five assessed, and Bacteroidetes was the least abundant. 


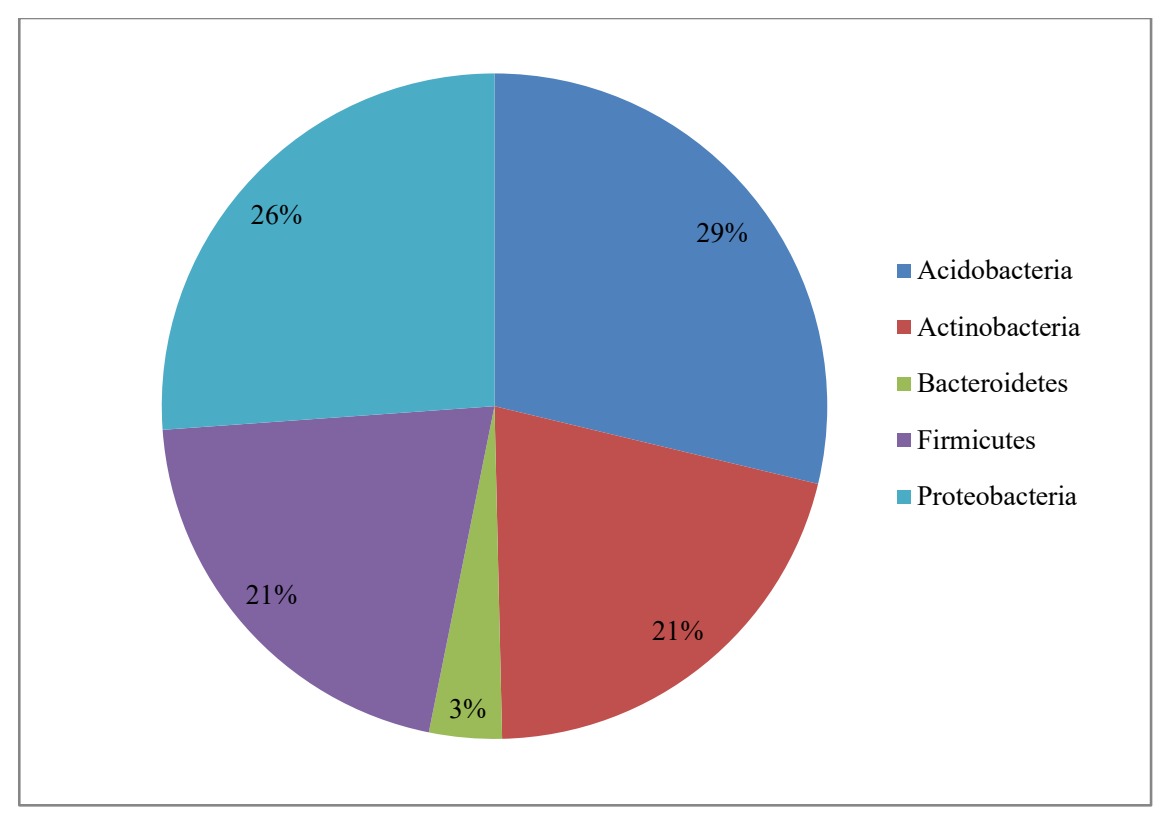

Figure 6-7 The overall average relative abundance of bacterial phyla in all the soil reactors.

The results showed little differences in calculated CFU equivalent (CCE) for each phylum between controls 1 and 2, as shown in Figure 6-8. The ANOVA analysis also indicated no major differences in the abundance of the studied phyla between controls 1 and 2, and the calculated p-values were 0.6, 0.52, 0.43, 0.84 and 0.04 for Acidobacteria, Actinobacteria, Bacteroidetes, Firmicutes, and Proteobacteria, respectively. This indicates that AgNPs at a concentration of $20 \mathrm{mg} \mathrm{AgNPs/g} \mathrm{soil} \mathrm{had} \mathrm{minimal} \mathrm{impact} \mathrm{on} \mathrm{the} \mathrm{presence} \mathrm{and} \mathrm{diversity} \mathrm{of}$ the assessed phyla, except for Proteobacteria which might be considered significantly affected by the AgNPs (p-value was 0.04). There was less than one Log10 CCE increase or decrease in each phylum in both controls 1 and 2. 


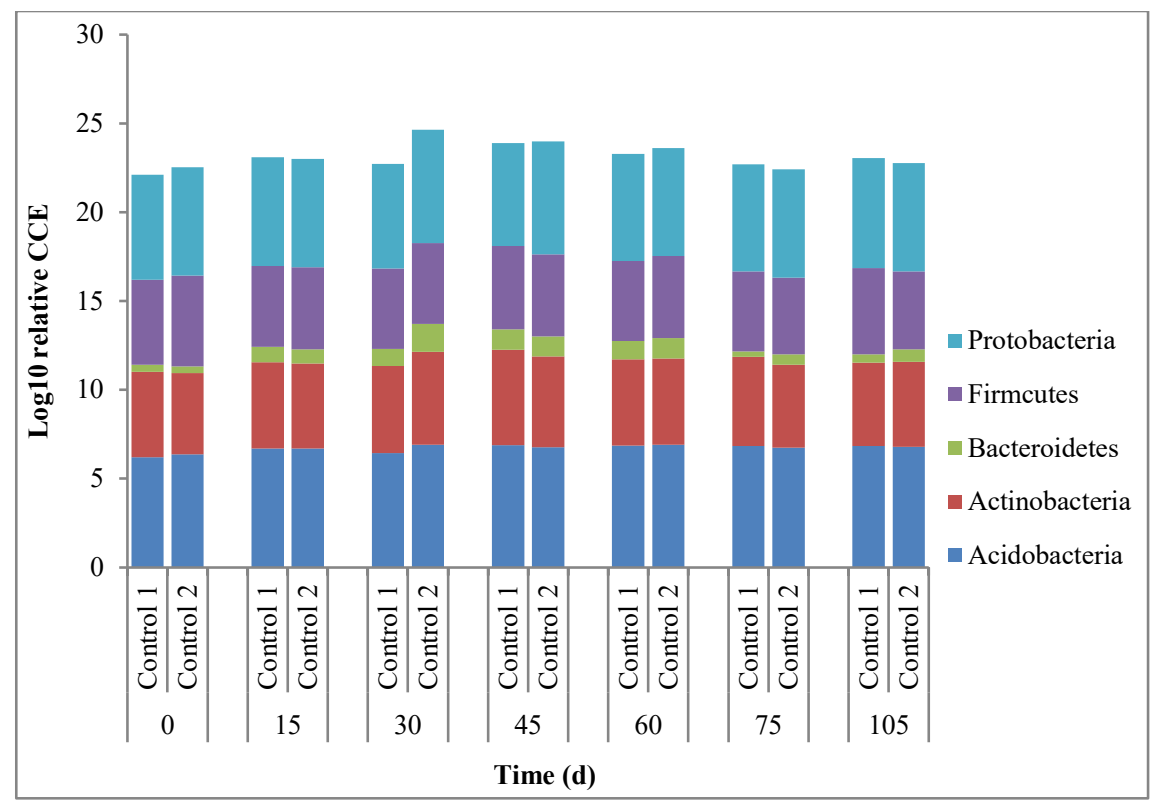

Figure 6-8 Relative change in CCE for the five phyla assessed in controls 1 and 2 over time.

\section{Acidobacteria CCE}

Acidobacteria was the only phyla that showed an increase in CCE in all soil reactors, as shown in Figure 6-9(a). This indicates that it can thrive in the presence of AgNPs and different sludge chemical treatments. The results and statistical analyses showed no major differences in CCE of Acidobacteria in all soil reactors, with a p-value ranging from 0.6 to 0.9. The ferric chloride reactor had the highest growth rate (an increase of $21 \%$ over time) for this phylum, while growth rates for the other soil reactors ranged from 4 to $10 \%$ over 105 days experiment duration.

\section{Actinobacteria CCE}

Actinobacteria did not show a significant increase or decrease in CCE in all reactors during the experiments, as shown in Figure 6-9(b), and ANOVA statistical analysis showed no substantial differences, as the p-value was higher than 0.05 between control 2 and the other reactors. All reactors followed similar trends with no major differences in CCE except for 
the ferric chloride treatment that seemed to promote Actinobacteria growth, with a $25 \%$ increase in $\mathrm{CCE}$ over the 105 days experiment time frame.

\section{Bacteroidetes CCE}

The relative CCE of Bacteroidetes showed no significant differences between control 2 and the other soil reactors, as shown in Figure 6-9(c). The results were analyzed using ANOVA and showed a p-value greater than 0.05. However, the Bacteroidetes CCE increased in the ferric chloride and alum reactors, decreased with the remaining treatment and was relatively similar in controls 1 and 2 . These results indicate that treatment of sludge with ferric and alum promoted the growth of this phylum in the soil reactors.

\section{Firmicutes CCE}

No significant differences were observed in the relative CCE of Firmicutes between controls 1 and 2, which indicate that AgNPs at this concentration had no substantial effect on the population of this phylum. However, as shown in Figure 6-9(d), control 1 remained relatively stable, with only a slight decrease on day 75 , followed by a slight increase, while control 2 decreased steadily throughout the experiment. The results of the relative CCE of Firmicutes showed that, compared to control 2, the alum reactor had the highest difference $(p-v a l u e=0.00023)$ followed by ferric chloride $(p-v a l u e=0.072)$. The polymer reactor had no significant difference with control $2(p-v a l u e=0.45)$. These results suggest that the type of chemicals used in sludge treatment had an impact on the population of this phylum. 


\section{Proteobacteria CCE}

Proteobacteria was the only phylum that had significant differences in the relative CCE between controls 1 and 2, as shown in Figure 6-9(e), indicating an adverse effect of the AgNPs on the Proteobacteria population in the soil. However, the p-value was 0.04, which was only slightly lower than $\alpha=0.05$ and control 1 increased by $5 \%$ compared to control 2 , which had no increase or decrease. The other soil reactors showed no significant differences in Proteobacteria CCE compared to control 2. However, there was a minor decrease of $1.6 \%$ in Proteobacteria CCE in the polymer reactor, and relatively slight increases from 1 to $7 \%$ in the remaining reactors. 

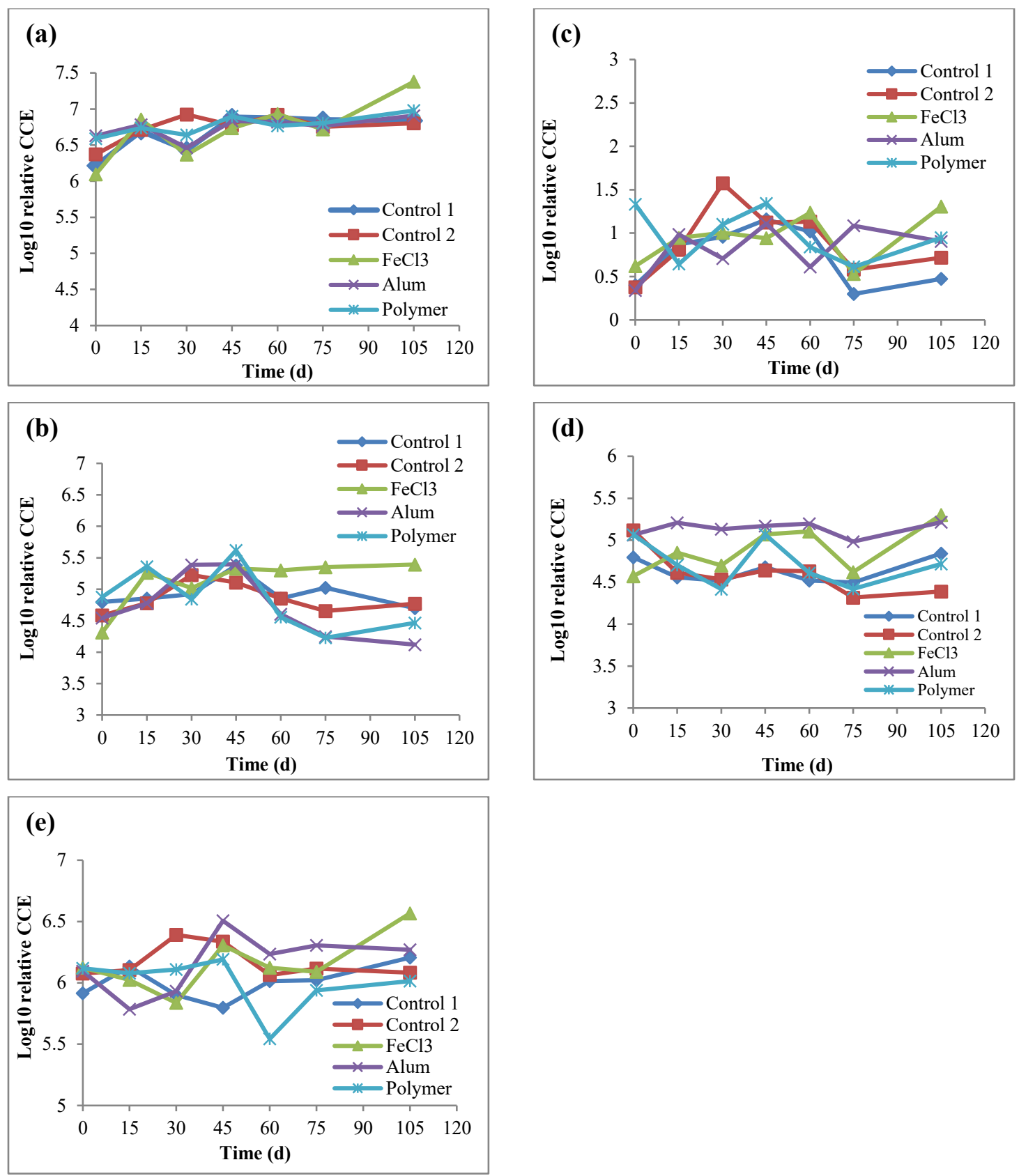

Figure 6-9 Relative change in CCE for Acidobacteria (a), Actinobacteria (b), Bacteroidetes (c), Firmicutes (d), Proteobacteria (e), in soil reactors over time.

Genomic DNA for all soil reactors was analyzed for the presence of the five most common phyla in soil and sludge using qPCR. In general, the results showed no significant difference in CCE for each phylum between controls 1 and 2 at each time point, indicating that AgNPs at this concentration had minimal effect on the presence and diversity of these 
phyla in the soil reactors over the experiment time (Figure 6-9). Some new findings were revealed when the presence and diversity of the five phyla assessed in the other soil reactors were analyzed (Figure 6-9). The first finding was that Acidobacteria CCE continued to increase, regardless of the presence of AgNPs and conditioning chemicals. This corresponds to what previous studies have shown that the phylum Acidobacteria have the highest tolerance to AgNPs (Yang et al., 2014). Furthermore, the change in each phylum over time under different sludge conditioners was not statistically significant, based on ANOVA analyses. In addition, ferric chloride treatment seemed to promote the growth of all phyla, while polymer treatment decreased the phyla over time, except Acidobacteria. This was expected, as iron plays a vital role in cell metabolism and polymers have toxicity and have been shown to enhance the antimicrobial activity of AgNPs (Kvitek et al., 2008). Thus, in this case, the impact on the soil phyla was more likely due to the type of sludge treatment, rather than the presence of AgNPs. Dissolution and release of silver ions $\left(\mathrm{Ag}^{+}\right)$ are believed to be the main cause of AgNPs toxicity (Xiu et al., 2012). AgNPs might have reacted with sludge constituents, or chemical added during treatment which likely used in the sludge treatment which lowered the silver toxicity. Many studies showed that sulfidation of $\mathrm{AgNPs}$ during different wastewater sludge treatment processes produce $\mathrm{Ag}_{2} \mathrm{~S}$ which is very stable and less toxic than AgNPs (Ma et al., 2013; Impellitteri et al., 2013; Wang et al., 2018). This less toxic form of silver has a less toxic impact on soil biological activity after land application of biosolids. Other studies also found that AgNPs toxicity could be caused by their very large surface area to volume ratio which enables them to easily penetrate the cell wall and potentially causes further damage by interacting with sulfur and phosphorus-containing compounds such as DNA, which can result in cell death. 
Toxicity can be reduced by changing the AgNPs behavior as seen when mixed with sludge; altering agglomeration of AgNPs into big flocs and their surface coating. In addition, the exposure time was considered as an important factor in the nanoparticles toxicity assessment. For example, AgNPs at a concentration range of 0.01-1 mg AgNPs/kg soil did not affect the soil biological activities over the short term; however, after a year of exposure (0.01 mg AgNPs/kg soil), the AgNPs were shown to significantly impact the soil microbial biomass, bacterial ammonia oxidizers and the abundance of nitrogen-fixing microorganisms (Grün et al., 2018).

\subsection{Conclusions}

This study evaluated the behavior and impact of AgNPs during the chemical conditioning of sludge, using three different chemicals, and their impact on soil bacterial activities after land application of biosolids. The main findings of this study were as follow:

1. Observation the TEM images showed that mixing AgNPs with sludge caused physical, chemical and morphological changes in AgNPs that were not observed in water, including coating, dissolution and agglomeration.

2. The three assessed conditioners; ferric chloride, alum, and synthetic polymers were effective in removing AgNPs from the liquid phase and concentrating them in sludge solids, which was confirmed by the negligible concentrations of AgNPs after performing the chemical conditioning of the sludge. However, the floc structure and incorporation of nanoparticles were different for each conditioner, as was examined by TEM imaging. 
3. After land application of biosolids at a ratio of $1 \%$, the results showed that the presence of AgNPs at $2 \mathrm{mg} \mathrm{AgNPs} / \mathrm{g}$ TS sludge (corresponding to $20 \mathrm{mg} \mathrm{AgNPs} / \mathrm{kg}$ soil) did not have a significant impact on the bacterial population in the soil, as represented by the five assessed phyla(Acidobacteria, Actinobacteria, Bacteroidetes, Firmicutes, and Proteobacteria)

4. Even though different trends of the five assessed phyla were observed over time corresponding to different chemicals used in sludge conditioning, these differences were not statistically significant.

5. AgNPs at the tested concentration did not also have a significant impact on HPC and the percentage of the live cells in the soil over time. 


\section{References}

APHA, A. P., American water works Association, A., \& Water environment federation, W. (2017). Standard methods for the examination of water and wastewater (23 ed.). (E. Rice, R. Baird, \& A. Eaton, Eds.) Washington, DC, USA: APHA, American Public Health Association; American water works Association, AWWA; Water environment federation, WEF.

Benn, T. M., \& Westerhoff, P. (2008). Nanoparticle silver released into water from commercially available sock fabrics. Environmental Science \& Technology, 42(11), 4133-4139.

Benn, T., Cavanagh, B., Hristovski, K., Posner, J. D., \& Westerhoff, P. (2010). The release of nanosilver from consumer products used in the home. Journal of Environmental Quality, 39(6), 1875-1882.

Blaser, S. A., Martin, S., Matthew, M., \& Konrad, H. (2008). Estimation of cumulative aquatic exposure and risk due to silver: contribution of nano-functionalized plastics and textiles. Science of The Total Environment, 390(2), 396-409.

Carbone, S., Antisari, L., Gaggia, F., Baffoni, L., Di Gioia, D., Vianello, G., \& Nannipieri, P. (2014). Bioavailability and biological effect of engineered silver nanoparticles in a forest soil. Journal of Hazardous Materials, 280, 89-96.

CCME, C. C. (2010). A Review of the Current Canadian Legislative Framework for Wastewater Biosolids. PN 1446, ISBN 978-1-896997-95-7 PDF.

City of Ottawa, C. o. (2014). City of Ottawa annual report. Ottawa, Canada: Citty of Ottawa.

Colman, B. P., Arnaout, C. L., Anciaux, S., Gunsch, C. K., Hochella Jr, M. F., Kim, B., . . al., e. (2013). Low Concentrations of Silver Nanoparticles in Biosolids Cause Adverse Ecosystem Responses under Realistic Field Scenario. PLOS One, 8(2), e57189.

Cumberland, S. A., \& Lead, J. R. (2009). Particle size distributions of silver nanoparticles at environmentally relevant conditions. Journal of Chromatography, 1216(52), 9099-9105.

Cunningham, S., Brennan-Fournet, M. E., Deirdre, L., Byrnes, L., \& Joshi, L. (2013). Effect of nanoparticle stabilization and physicochemical properties on exposure outcome: acute 
toxicity of silver nanoparticle preparations in zebrafish (Danio rerio). Environmental Science \& Technology, 47(8), 3883-3892.

EPA, U. E. (2018). Standards for the use or disposal of sewage sludge. Washington, DC, US: EPA40 CFR 503.

Fierer, N., Jackson, J. A., Vilgalys, R., \& Jackson, R. B. (2005). Assessment of soil microbial community structure by use of taxon-specific quantitative PCR assays. Appl. Environ. Microbiol., 71(7), 4117-4120.

Geranio, L., Manfred, H., \& Bernd, N. (2009). The behavior of silver nanotextiles during washing. Environmental Science \& Technology, 43(21), 8113-8118.

Gottschalk, F., Tobias, S., Roland W., S., \& Bernd, N. (2009). Modeled environmental concentrations of engineered nanomaterials ( $\mathrm{TiO} 2, \mathrm{ZnO}, \mathrm{Ag}, \mathrm{CNT}$, fullerenes) for different regions. Environmental Science \& Technology, 43(24), 9216-9222.

Grün, A.-L., Straskraba, S., Schulz, S., Schloter, M., \& Emmerling, C. (2018). Long-term effects of environmentally relevant concentrations of silver nanoparticles on microbial biomass, enzyme activity, and functional genes involved in the nitrogen cycle of loamy soil. Journal of Environmental Sciences, 69, 12-22.

Hänsch, M., \& Emmerling, C. (2010). Effects of silver nanoparticles on the microbiota and enzyme activity in soil. Journal of Plant Nutrition and Soil Science, 173(4), 554-558.

Impellitteri, C. A., Harmon, S., Silva, R. G., Miller, B. W., Scheckel, K. G., Luxton, T. P., . . . Panguluri, S. (2013). Transformation of silver nanoparticles in fresh, aged, and incinerated biosolids. Water Research, 47(12), 3878-3886.

Johansson, M., Stenberg, B., \& Torstensson, L. (1999). Microbiological and chemical changes in two arable soils after long-term sludge amendments. Biology and Fertility of Soils, 30(12), 160-167.

Kaegi, R., Sinnet, B., Zuleeg, S., Hagendorfer, H., Mueller, E., Vonbank, R., . . B Burkhardt, M. (2010). Release of silver nanoparticles from outdoor facades. Environmental Pollution, 158(9), 2900-2905. 
Kaegi, R., Voegelin, A., Sinnet, B., Zuleeg, S., Hagendorfer, H., Burkhardt, M., \& Siegrist, H. (2011). Behavior of metallic silver nanoparticles in a pilot wastewater treatment plant. Environmental Science \& Technology, 45(9), 3902-3908.

Kumar, N., Shah, V., \& Walker, V. K. (2011). Perturbation of an arctic soil microbial community by metal nanoparticles. Journal of Hazardous Materials, 190(1-3), 816-822.

Kvitek, L., Panáček, A., Soukupova, J., Kolář, M., Večeřová, R., Prucek, R., . . Zbořil, R. (2008). Effect of surfactants and polymers on stability and antibacterial activity of silver nanoparticles (NPs). The Journal of Physical Chemistry C, 112(15), 5825-5834.

Levard, C., Reinsch, B. C., Michel, F. M., Oumahi, C., Lowry, G. V., \& Brown Jr., G. E. (2011). Sulfidation processes of PVP-coated silver nanoparticles in aqueous solution: impact on dissolution rate. Environmental Science \& Technology, 45(12), 5260-5266.

Li, X., Lenhart, J. J., \& Walker, H. W. (2010). Dissolution-accompanied aggregation kinetics of silver nanoparticles. Langmuir, 26(22), 16690-16698.

Liang, Z., Das, A., \& Hu, Z. (2010). Bacterial response to a shock load of nanosilver in an activated sludge treatment system. Water Research, 44(18), 5432-5438.

Lombi, E., Donner, E., Taher, S., Tavakkoli, E., Jämting, Å. K., McClure, S., . . Vasilev, K. (2013). Transformation of four silver/silver chloride nanoparticles during anaerobic treatment of wastewater and post-processing of sewage sludge. Environmental Pollution, 176, 193-197.

Ma, R., Levard, C., Judy, J. D., Unrine, J. M., Durenkamp, M., Martin, B., . . Lowry, G. V. (2013). Fate of zinc oxide and silver nanoparticles in a pilot wastewater treatment plant and in processed biosolids. Environmental Science \& Technology, 48(1), 104-112.

Masrahi, A., VandeVoort, A. R., \& Arai, Y. (2014). Effects of silver nanoparticle on soilnitrification processes. Archives of Environmental Contamination and Toxicology, 66(4), 504-513.

Metcalf, L., \& Eddy, H. P. (2003). Waste Water Engineering: Treatment and Reuse (4 ed.). New York: McGraw-Hill. 
Morales, S. E., \& Holben, W. E. (2009). Empirical testing of 16S rRNA gene PCR primer pairs reveals variance in target specificity and efficacy not suggested by in silico analysis. Applied and Environmental Microbiology, 75(9), 2677-2683.

OME, O. M., \& OMAFR, O. M. (1996). Guidelines for The Utilization of Biosolid and Other Waste on Agricultural lands. Ontario, Canada: Ontario.

Panacek, A., Kolar, M., Vecerova, R., Prucek, R., Soukupova, J., Krystof, V., . . Kvitek, L. (2009). Antifungal activity of silver nanoparticles against Candida spp. Biomaterials, 30(31), 6333-6340.

Peretyazhko, T. S., Zhang, Q., \& Colvin, V. L. (2014). Size-controlled dissolution of silver nanoparticles at neutral and acidic $\mathrm{pH}$ conditions: kinetics and size changes. Environmental Science \& Technology, 48(20), 11954-11961.

Pfeiffer, S., Milica, P., Birgit, M., Kathrin, L., Evelyn, H., Paul, L., . . Angela, S. (2014). Improved group-specific primers based on the full SILVA 16S rRNA gene reference database. Environmental Microbiology, 16(8), 2389-2407.

Rebecca, B. (2004). Soil survey laboratory method manual. Soil Survey Laboratory Investigations Report.

Samarajeewa, A., Velicogna, J., Princz, J., Subasinghe, R., Scroggins, R., \& Beaudette, L. (2017). Effect of silver nano-particles on soil microbial growth, activity and community diversity in a sandy loam soil. Environmental Pollution, 220, 504-513.

Schlich, K., Beule, L., \& Hund-Rinke, K. (2016). Single versus repeated applications of CuO and Ag nanomaterials and their effect on soil microflora. Environmental Pollution, 215, 322330.

Schlich, K., Klawonn, T., Terytze, K., \& Hund-Rinke, K. (2013). Hazard assessment of a silver nanoparticle in soil applied via sewage sludge. Environmental Sciences Europe, 25(1), 17.

Schwertfeger, D. M., Velicogna, J. R., Jesmer, A. H., Saatcioglu, S., McShane, H., Scroggins, R. P., \& Princz, J. I. (2017). Extracting Metallic Nanoparticles from Soils for Quantitative Analysis: Method Development Using Engineered Silver Nanoparticles and SP-ICP-MS. Analytical Chemistry, 89(4), 2505-2513. 
Shafer, M. M., Joel T., O., \& David E., A. (1998). Removal, partitioning, and fate of silver and other metals in wastewater treatment plants and effluent-receiving streams. Environmental Toxicology and Chemistry, 17(4), 630-641.

Shakya, M., Christopher, Q., James H., C., Zamin K., Y., Christopher W., S., \& Mircea, P. (2013). Comparative metagenomic and rRNA microbial diversity characterization using archaeal and bacterial synthetic communities. Environmental Microbiology, 15(6), 1882-1899.

Shammas, N. K., \& Wang, L. K. (2007). Land Application of Biosolids. In L. K. Wang, N. K. Shammas, \& Y.-T. Hung (Eds.), Biosolids Treatment Processes (pp. 705-745). Totowa, N.J: Humana Press, Springer.

Shoults-Wilson, W. A., Reinsch, B. C., Tsyusko, O. V., Bertsch, P. M., Lowry, G. V., \& Unrine, J. M. (2011). Role of particle size and soil type in toxicity of silver nanoparticles to earthworms. Soil Science Society of America Journal, 75(2), 365-377.

Svec, D., Ales, T., Vendula, N., Michael W., P., \& Mikael, K. (2015). How good is a PCR efficiency estimate: Recommendations for precise and robust qPCR efficiency assessments. Biomolecular Detection and Quantification, 3, 9-16.

Thalmann, B., Voegelin, A., Morgenroth, E., \& Kaegi, R. (2016). Effect of humic acid on the kinetics of silver nanoparticle sulfidation. Environmental Science: Nano , 3(1), 203-212.

Tseng, K.-H., Liao, C.-Y., Tien, D.-C., \& Tsung, T.-T. (2009). Dissociation of Colloidal Silver into Ionic Form through Membrane under Electric Field. In S. Soomro (Ed.), Engineering the Computer Science and IT (pp. 193-212). Croatia: InTech.

Vance, M. E., Kuiken, T., Vejerano, E. P., McGinnis, S. P., Hochella Jr, M. F., Rejeski, D., \& Hull, M. S. (2015). Nanotechnology in the real world: Redeveloping the nanomaterial consumer products inventory. Beilstein Journal of Nanotechnology, 6, 1769.

Větrovský, T., \& Baldrian, P. (2013). The variability of the 16S rRNA gene in bacterial genomes and its consequences for bacterial community analyses. PloS One, 8(2), e57923.

Wang, P., Lombi, E., Menzies, N. W., Zhao, F.-J., \& Kopittke, P. M. (2018). Engineered silver nanoparticles in terrestrial environments: a meta-analysis shows that the overall environmental risk is small. Environmental Science: Nano, 5(11), 2531-2544. 
Whitley, A. R., Levard, C., Oostveen, E., Bertsch, P. M., Matocha, C. J., Kammer, F. v., \& Unrine, J. M. (2013). Behavior of Ag nanoparticles in soil: effects of particle surface coating, aging and sewage sludge amendment. Environmental Pollution, 182, 141-149.

Xiu, Z.-m., Zhang, Q.-b., Puppala, H. L., Colvin, V. L., \& Alvarez, P. J. (2012). Negligible particlespecific antibacterial activity of silver nanoparticles. Nano Letters, 8(12), 4271-4275.

Yang, Y., Quensen, J., Mathieu, J., Wang, Q., Wang, J., Li, M., . . . Alvarez, P. J. (2014). Pyrosequencing reveals higher impact of silver nanoparticles than $\mathrm{Ag}+$ on the microbial community structure of activated sludge. Water Research, 48, 317-325.

Zhang, W., Xiao, B., \& Fang, T. (2018). Chemical transformation of silver nanoparticles in aquatic environments: Mechanism, morphology and toxicity. Chemosphere, 191, 324-334. 


\section{Supplementary data}

\section{Coagulation-flocculation preliminary experiment}

Preliminary coagulation-flocculation experiments were done to find the optimum chemical dose. Four different doses of each chemical were added to stirred sludge and a conditioning treatment was performed according to a known protocol as mentioned in Section 6.2.3. The evaluation of the conditioning treatment was based on the capillary suction test (CST) for the conditioned sludge and the turbidity of the supernatant. Capillary suction time (CST) was measured for the conditioned sludge at the end of the experiments by placing $10 \mathrm{~mL}$ of the conditioned sludge in the CST reservoir. CST was measured for each sample in three replicates and the average of the readings was used. The turbidity of the supernatant was measured after the settling period, using an HACH 2100AN turbidimeter.

Table S-1 CST and turbidity of the conditioned sludge.

\begin{tabular}{|c|c|c|c|}
\hline \multirow{3}{*}{ Chemical } & $\begin{array}{c}\text { Chemical/ TS of sludge } \\
(\mathbf{g} / \mathbf{g})\end{array}$ & CST (s) & $\begin{array}{c}\text { Turbidity } \\
\text { (NTU) }\end{array}$ \\
\hline \multirow{3}{*}{$\mathrm{FeCl}_{3}$} & 0.4 & 331 & 272 \\
\cline { 2 - 4 } & 0.8 & 119.1 & 198 \\
\cline { 2 - 4 } & 0.1 & 41.8 & 64 \\
\hline \multirow{3}{*}{ Alum } & 1.3 & 97 & 66 \\
\cline { 2 - 4 } & 0.18 & 436.3 & 280 \\
\cline { 2 - 4 } & 0.36 & 194.2 & 170 \\
\hline \multirow{5}{*}{ Polymer } & 0.7 & 64 & 62 \\
\cline { 2 - 4 } & 1.4 & 120 & 73 \\
\cline { 2 - 4 } & 0.0026 & 191.2 & 124 \\
\cline { 2 - 4 } & 0.0052 & 11.1 & 60 \\
\hline
\end{tabular}


Total genomic soil DNA concentration

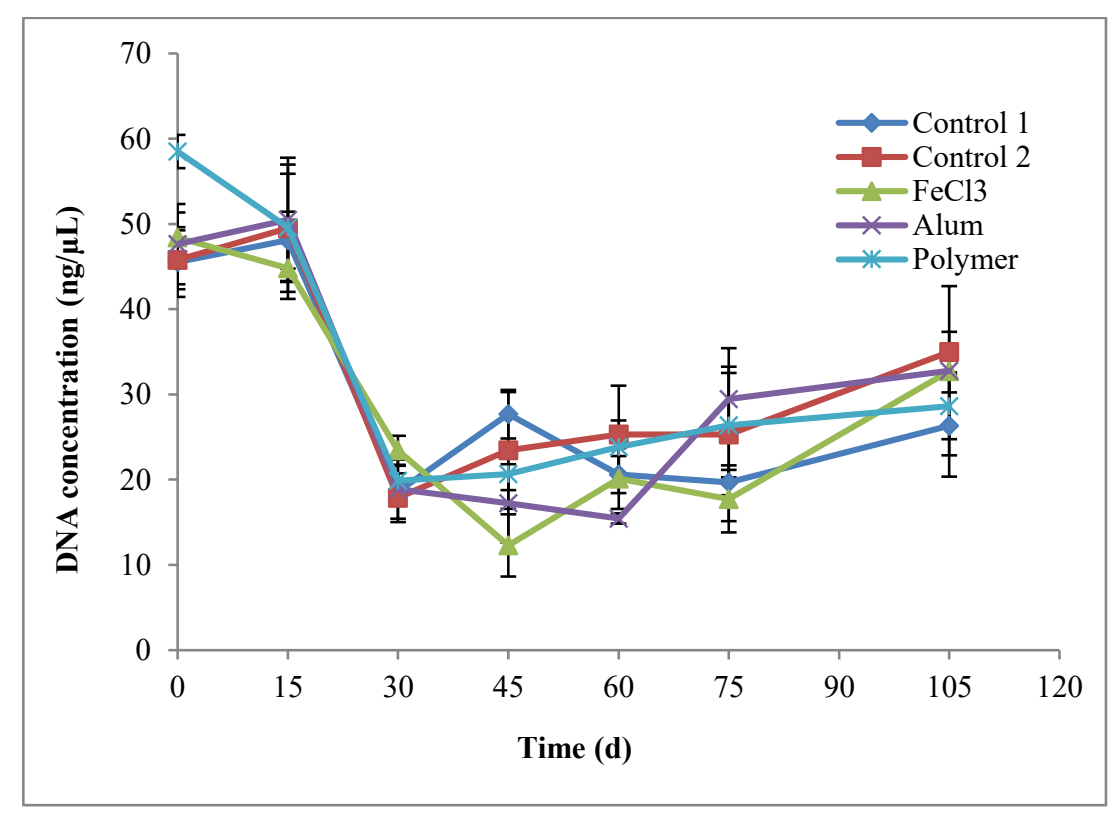

Figure S-1 Genomic DNA concentrations over time. 


\section{Live/Dead images}
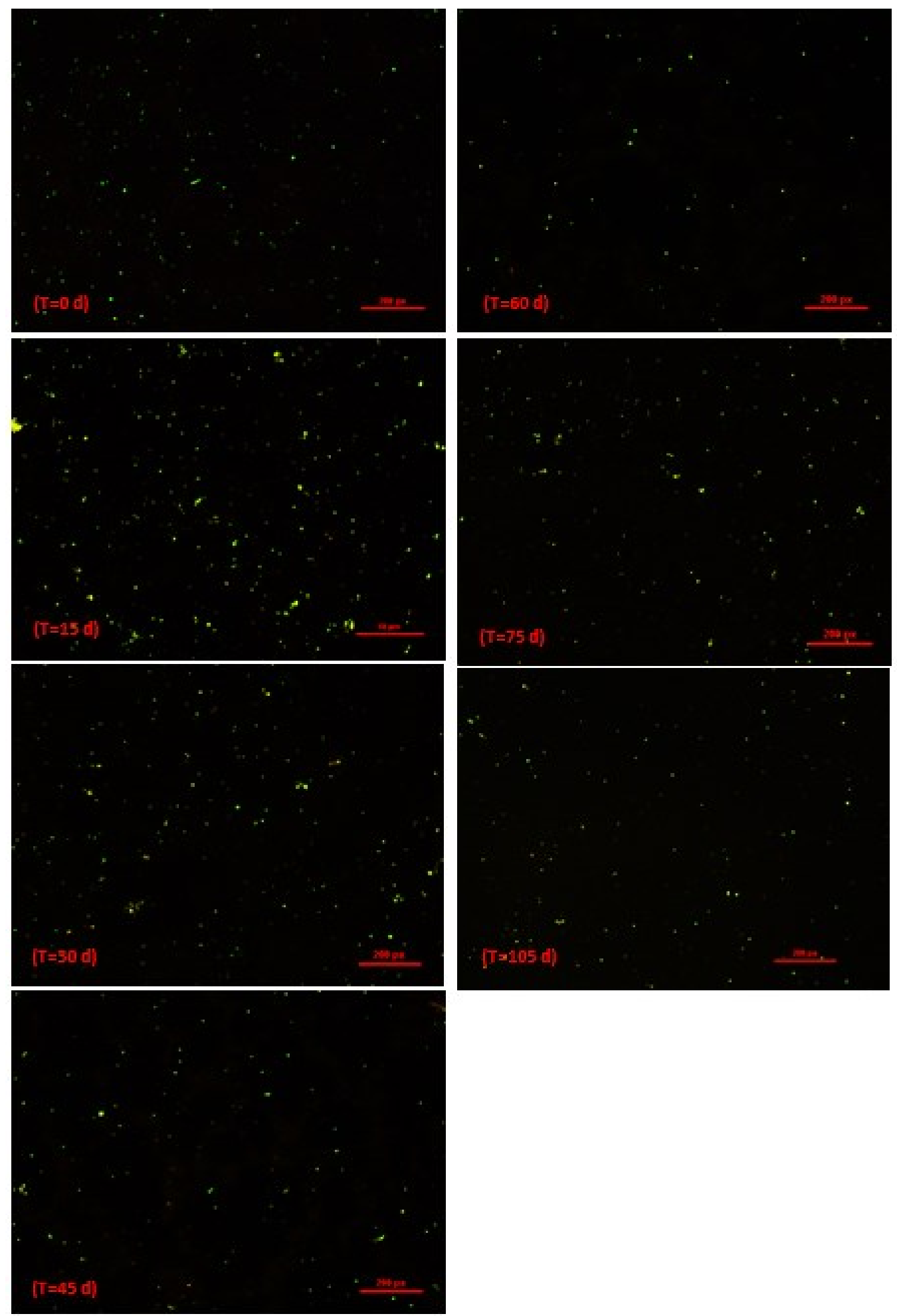

Figure S-2 Live/Dead images of control 1. 


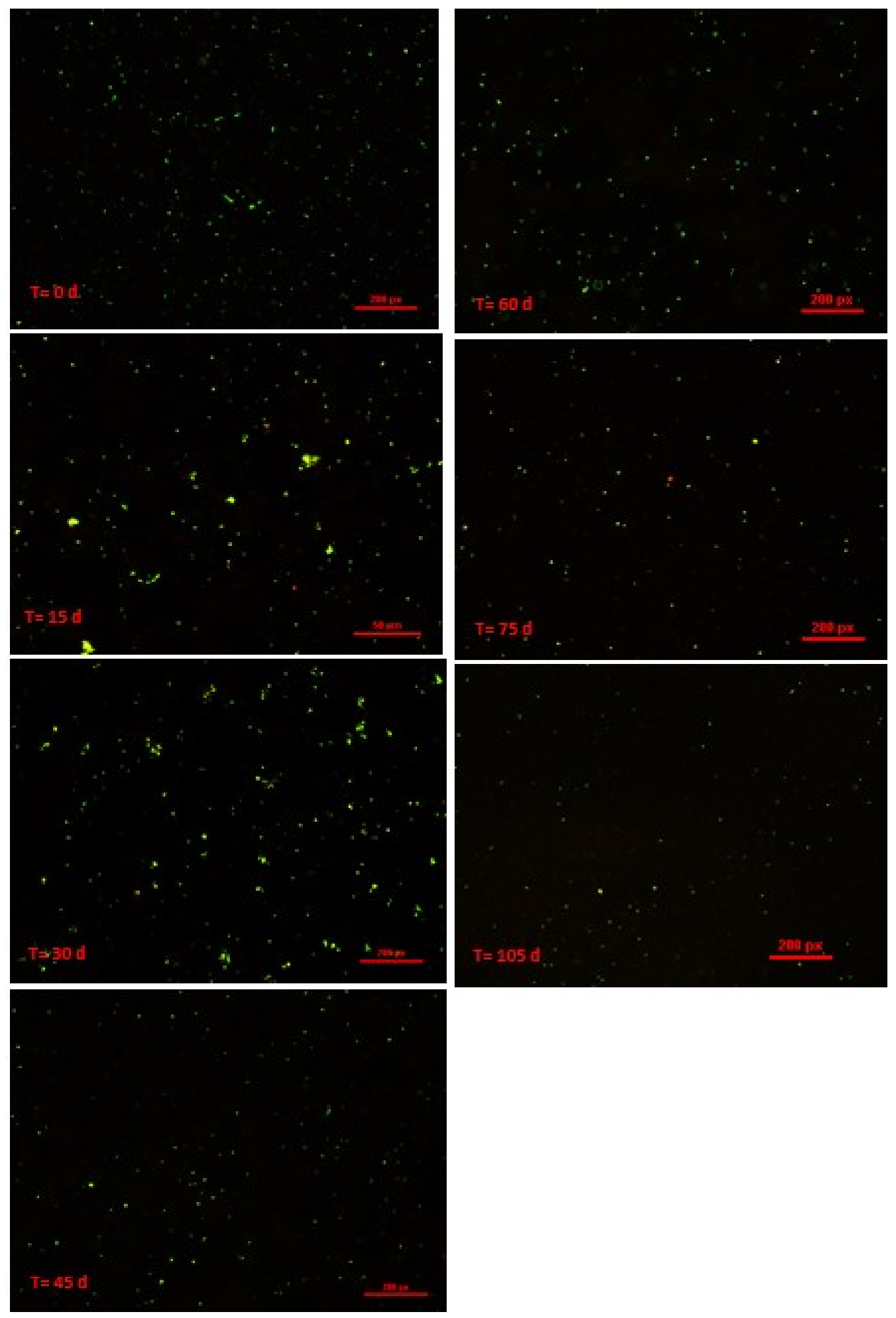

Figure S-3 Live/Dead images of control 2. 


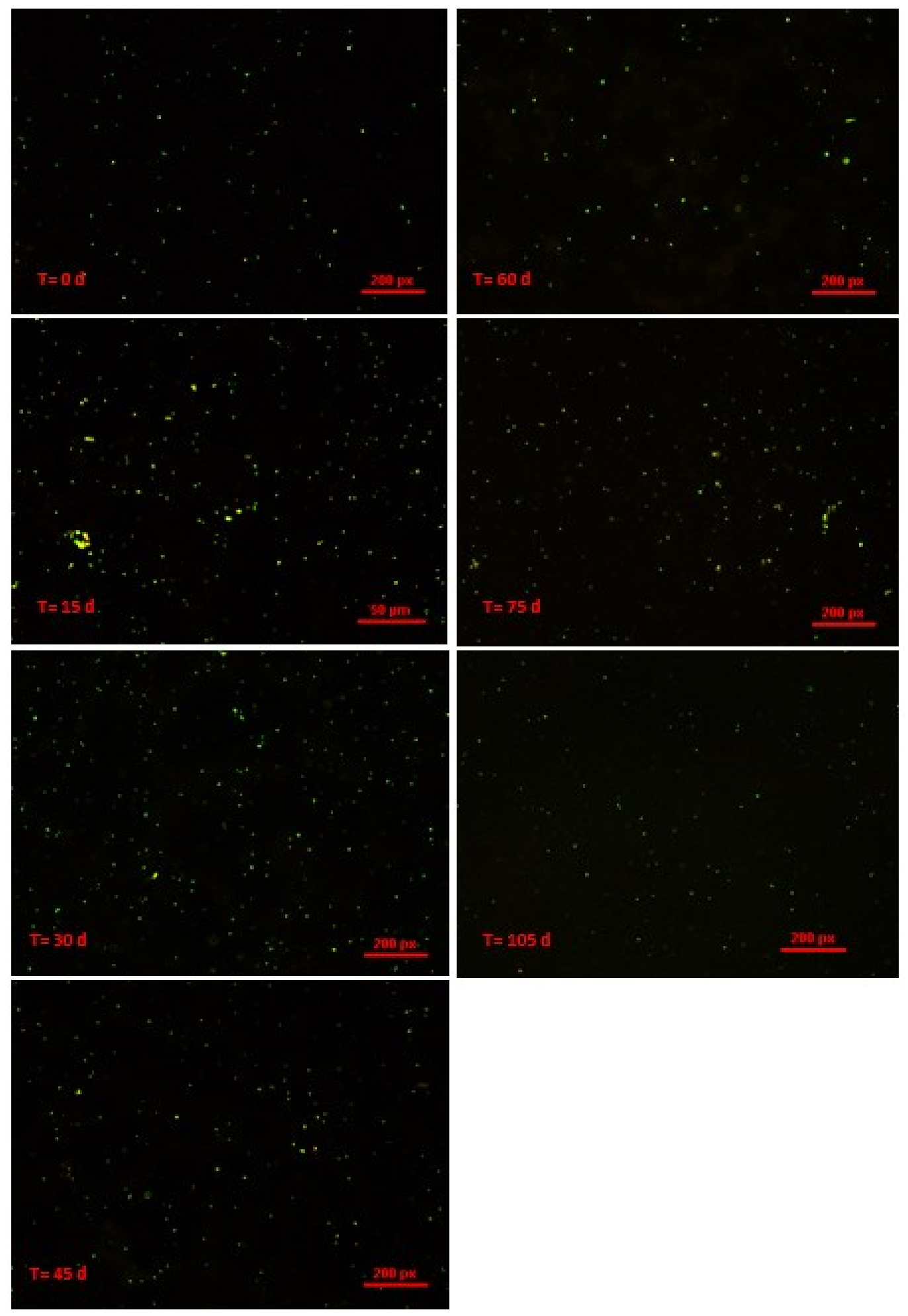

Figure S-4 Live/Dead images of $\mathrm{FeCl}_{3}$ reactor. 


$$
\text { | }
$$



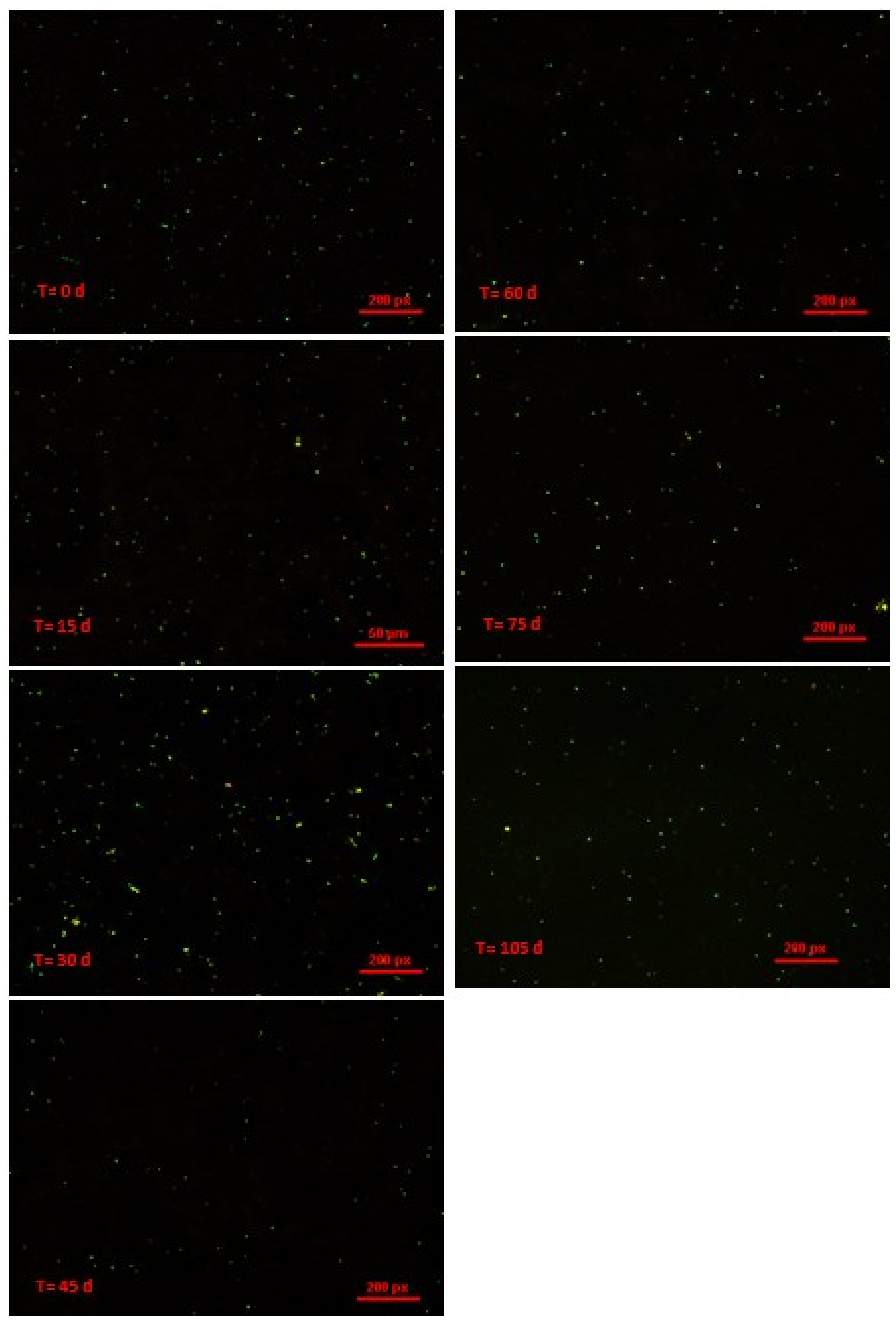

Figure S-6 Live/Dead images of polymer reactor. 


\title{
Chapter 7: Transformation of Silver Nanoparticles (AgNPs) during Lime Treatment of Sludge and their Impact on Soil Bacteria after Land Application
}

\begin{abstract}
The impact of silver nanoparticles (AgNPs) on soil microorganisms is an important concern. Due to extensive antibacterial applications, AgNPs have been released into wastewater and subsequently into the ensuing sludge. Land application of biosolids (treated sludge) leads to the release of AgNPs into the soil, which can adversely affect the bacteria necessary for soil and plant health. This study evaluates changes in the population and diversity of the five most relevant bacterial phyla found in soil after application of limestabilized sludge containing AgNPs. It also investigates the impact of lime stabilization on the behavior of AgNPs. The study was performed by mixing an environmentally relevant concentration of AgNPs (2 mg AgNPs/g TS) with sludge, applying lime stabilization of sludge, and simulating the land application of biosolids. Transmission electron microscopy (TEM) and associated energy-dispersive $\mathrm{x}$-ray spectroscopy (EDS) were used to investigate the morphological and compositional changes of AgNPs during lime stabilization. After application of lime stabilized sludge to the soil, soil samples were periodically analyzed for total genomic DNA, and for changes in bacterial phyla diversity using quantitative polymerase chain reaction (qPCR). The results showed that sludge lime treatment removed AgNPs from the aqueous phase and AgNPs were deposited on the lime molecules. The results revealed that AgNPs did not significantly impact the presence and diversity of the assessed phyla in the soil. However, lime stabilized sludge with AgNPs
\end{abstract}


affected the abundance of each phylum over time. The impact of AgNPs on the phyla abundance could be attributed to several factors, including the interactions between sludge constituents and AgNPs or lime.

Keywords: silver nanoparticles, sludge, lime stabilization, land application of biosolids, Bacterial phyla

\subsection{Introduction}

The nanoparticle industry is growing exponentially, and new nanomaterials and products are being introduced to consumers on an almost daily basis. AgNPs are commonly used as antimicrobial agents and incorporated in a wide range of merchandise and applications. Many products such as textiles, antimicrobial coatings, keyboards, wound dressings, and biomedical devices contain AgNPs that continually release a low level of silver ions to protect against potentially harmful microorganisms (POS, 2019). However, AgNPs can easily bleed out of textiles in just a few washing cycles (Benn \& Westerhoff, 2008; Geranio et al., 2009; Lorenz et al., 2012). Geranio et al. (2009) also found that just one wash cycle can increase the total amount of silver released from $1 \%$ to $45 \%$, depending on the type of fabric and the manufacturing methods. Thus, a large portion of these AgNPs likely ends up in wastewater treatment plants (Blaser et al., 2008; Gottschalk et al., 2009). In addition, several studies have revealed the toxicity of AgNPs on different microorganisms that are necessary for wastewater treatment (Masrahi et al., 2014; Liang et al., 2010).

The sewer system is considered a primary source for the release of AgNPs into the environment. AgNPs participate in a wide range of physical, chemical and biological reactions, both after they are introduced into the sewer system and during wastewater and 
sludge treatment (Nielsen et al., 2006, Fabrega et al., 2009). Although dissolution of AgNPs has been observed, association with solids is reportedly the primary removal mechanism from wastewater (Shafer et al., 1998; Kaegi et al., 2011). More than 94\% of AgNPs entering wastewater treatment plants is associated with sludge solids after treatment (Shafer et al., 1998).

Treated sludge, also known as biosolids, is rich in nutrients essential for plant growth, including carbon, nitrogen and phosphorous, and they are applied to improve soil properties such as texture and water holding capacity (Metcalf \& Eddy, 2003; Shammas \& Wang, 2007). However, studies have shown that land application of biosolids can add harmful substances to soil as well (Erland et al., 1998). Some studies also showed that the antimicrobial activity of AgNPs could have adverse effects on soil microorganisms, and possibly affect the organisms necessary for plant growth (McGee et al., 2017; Dimkpa et al., 2012).

Lime stabilization is a widely used sludge treatment method, as it is easy to apply, inexpensive, and beneficial for agriculture (Metcalf \& Eddy, 2003; Clint et al., 2007). In addition to chemical stabilization, lime may also be used as a conditioning chemical during treatment to achieve coagulation and flocculation (Turovskiy \& Mathai, 2006). Lime stabilization works by raising sludge $\mathrm{pH}$ to 12 for at least two hours, which terminates microbial activity and allows small particles to coagulate into larger particles that can easily be separated from the aqueous phase. Lime is a powerful chemical that can impact the physical, chemical and morphological characteristics of AgNPs in sludge, and thereby change their impact on soil biological activity. It was shown that $\mathrm{Ag}_{2} \mathrm{~S}$ was the dominant silver compound found after the heat and lime treatment of sludge, with no $\operatorname{Ag}(0)$ observed 
(Ma et al., 2013). Applying AgNPs to soil through sludge may be different from applying them directly. Certain properties of AgNPs that may affect their behavior and have an impact on soil may be altered or eliminated after interaction with sludge or have undergone different sludge treatment processes. For example, Whitley et al. (2013) demonstrated that the effect of the surface coating of AgNPs in soil matrices in terms of colloidal stability was annulled when AgNPs were applied with sewage sludge. Additionally, some studies investigated the impact of AgNPs on soil applied with biosolids after sludge treatment. The study intended to examine how lime stabilization affects the transformation of AgNPs in sludge and its impact on the soil biological activity and overall health. Soil biological activity was investigated by evaluating the change in the population and diversity of five prevalent bacterial phyla in soil.

\subsection{Materials and methods}

\subsubsection{Sludge characteristics}

Anaerobically digested sludge was collected from mesophilic anaerobic digesters and obtained from Robert O. Pickard Environmental Center (ROPEC); it is the central wastewater treatment plant located in Ottawa, Canada. It receives about $390 \mathrm{ML} / \mathrm{d}$ of wastewater from residential commercial and industrial sources (City of Ottawa, 2014). The chemical properties are provided in Table $7-1$. Sludge was stored at $4^{\circ} \mathrm{C}$ until use. 
Table 7-1 Anaerobically digested sludge characteristics.

\begin{tabular}{|l|l|}
\hline TP (total phosphorus) & $122 \pm 8 \mathrm{mg} / \mathrm{L}$ \\
\hline TN (total nitrogen) & $1043.3 \pm 33 \mathrm{mg} / \mathrm{L}$ \\
\hline TS (total solids) & $17720 \pm 95 \mathrm{mg} / \mathrm{L}$ \\
\hline TVS (total volatile solids) & $9803.3 \pm 45 \mathrm{mg} / \mathrm{L}$ \\
\hline pH & $7.4 \pm 0.1$ \\
\hline
\end{tabular}

Values are the mean of three replicate measurements \pm standard deviation.

\subsubsection{AgNPs characteristics}

A stock suspension of pristine AgNPs in water with a purity of $99.9 \%$, properties shown in Table 7-2, were purchased from nanoComposix Inc. (San Diego, CA, USA). The particle size distribution of AgNPs was examined and confirmed by TEM analysis. AgNPs suspension stock solution was maintained at $4^{\circ} \mathrm{C}$ until use.

Table 7-2 AgNPs characteristics.

\begin{tabular}{|l|l|}
\hline Diameter (TEM) & $23.1 \pm 6.9 \mathrm{~nm}$ \\
\hline Coefficient of variation & $29.8 \%$ \\
\hline Surface area (TEM) & $21.5 \mathrm{~m}^{2} / \mathrm{g}$ \\
\hline Particle concentration & $7.2 \times 10^{13} \mathrm{particles} / \mathrm{mL}$ \\
\hline Hydrodynamic diameter & $49.6 \mathrm{~nm}$ \\
\hline Zeta potential & $-30.6 \mathrm{mV}$ \\
\hline pH of the solution & 6.3 \\
\hline Particle surface coating & Polyvinylpyrrolidone (PVP) \\
\hline Solvent & Milli-Q water \\
\hline
\end{tabular}




\subsubsection{Lime treatment}

Quicklime $(\mathrm{CaO})$ with a molecular weight of $56.08 \mathrm{~g} / \mathrm{mol}$, was purchased from SigmaAldrich. A lime slurry was prepared by mixing powder lime with water at a concentration of $150 \mathrm{mg} / \mathrm{mL}(15 \%)$. The lime slurry was then mixed with anaerobically digested sludge at a concentration that maintained the $\mathrm{pH}$ above 12 for $2 \mathrm{~h}$.

\subsubsection{Soil characteristics}

Loamy topsoil was used in this study; it was free from additions such as compost or chemical fertilizer. It was obtained from Artistic Landscape Designs Ltd. The soil was processed through a sieve with $2 \mathrm{~mm}$ openings, air dried and stored at $4^{\circ} \mathrm{C}$ in the fridge until use. Initial soil analysis included: total organic carbon (TOC), total nitrogen (TN), phosphorus, pH, and cation exchange capacity (CEC) and are shown in Table 7-3.

\section{Table 7-3 Soil characteristics.}

\begin{tabular}{|l|l|}
\hline TOC & $4248 \pm 12 \mathrm{mg} / \mathrm{kg}$ \\
\hline TN & $184 \pm 7 \mathrm{mg} / \mathrm{kg}$ \\
\hline TP & $13 \pm 2 \mathrm{mg} / \mathrm{kg}$ \\
\hline pH & $6.6 \pm 0.7$ \\
\hline CEC & $30.8 \pm 2 \mathrm{meq} / 100 \mathrm{~g}$ \\
\hline
\end{tabular}

Values are the mean of three replicate measurements \pm standard deviation.

\subsubsection{Experimental design}

This study involved two stages. The first stage investigated the effect of sludge lime treatment on the fate and behavior of AgNPs in sludge. Lime treatment was conducted on two different test matrices; nanopure water and anaerobically digested sludge. Nanopure 
water was used as a control matrix to observe the interaction between AgNPs and lime in the absence of other contaminants. There were two test reactors for both nanopure water and sludge, one was the control, which had no lime treatment, and the other reactor was subjected to lime treatment. Each reactor contained $5 \mathrm{~mL}$ of water or sludge with two replicates each. AgNPs were added to each reactor at a concentration of $0.1 \mathrm{mg} / \mathrm{mL}$ and vortexed for $30 \mathrm{~s}$. Lime stabilization was performed by adding lime to each reactor while continuously measuring the $\mathrm{pH}$ until a $\mathrm{pH}$ of 12 was reached and maintained for two hours, as shown in Table S-1. After lime treatment, water and sludge test reactor samples were prepared for TEM analysis by placing approximately $5 \mu \mathrm{L}$ of the water and conditioned sludge on separate $3.05 \mathrm{~mm}, 200$ mesh (grid hole size $\approx 97 \mathrm{~nm}$ ) formvar/carbon-coated support copper grid film from Ted Pella Inc. Three replicates were done for each sample, and after preparation, the samples were analyzed using FEI Tecnai G2 F20 (Netherlands) at the Nano Imaging Facility (NIF), Carleton University. In addition to morphological analyses, the elemental composition of the samples was analyzed using EDS coupled to TEM. This combination allows EDS to provide an elemental composition of an area that is only a few nanometers in diameter. The results are shown as a plot of the emitted x-rays versus their energy in $\mathrm{keV}$. The plot has many peaks, each corresponds to specific elements.

The second stage of the study investigated the effect of AgNPs on the relative population of selected bacterial phyla in soil reactors representing the land application. Test reactors were made in $250 \mathrm{~mL}$ clean glass beakers filled with $100 \mathrm{~g}$ dry soil. Each soil-filled reactor received sludge that was previously mixed with AgNPs. There were three reactors, and two replicates for each reactor. There were two control reactors: control 1 received untreated anaerobically digested sludge without AgNPs, while control 2 received the same sludge 
previously mixed with AgNPs. The other reactor received sludge mixed with AgNPs and subjected to lime treatment. The final soil moisture content was $60 \%$ at the start of the experiment. The sludge/soil ratio was $0.01 \mathrm{~g} / \mathrm{g}$ (dry weight), and it was selected based on the biosolids land application guidelines at a rate of 8 ton/ha every 5 years (OME \& OMAFRA, 1996). Also, other studies were considered in choosing the sludge application ratio (Colman et al., 2013; Whitley et al., 2013; Johansson et al., 1999). The applied sludge was previously spiked with AgNPs suspension to obtain a final concentration of $2 \mathrm{mg}$ AgNPs/g sludge (dry weight), that corresponded to $20 \mathrm{mg} \mathrm{AgNPs/kg} \mathrm{soil} \mathrm{when} \mathrm{sludge} \mathrm{was}$ combined with soil. The AgNPs concentration used represents the higher range published values of their recorded and predicted concentrations in wastewater sludge (CCME, 2010; EPA, 2018; Blaser et al., 2008; Gottschalk et al., 2013). The reactors were incubated with day/night cycles of $8 / 16 \mathrm{~h}$ at a constant temperature of $22 \pm 1^{\circ} \mathrm{C}$. This study was carried out throughout $105 \mathrm{~d}$, and chemical and biological analyses were conducted every $15 \mathrm{~d}$ to examine the soil activity. TOC and $\mathrm{pH}$ were monitored every $15 \mathrm{~d}$. Moisture content was monitored every three days and maintained at $45 \%$ by addition of sterile distilled water. The chemical analyses involved cation exchange capacity (CEC), $\mathrm{pH}$, total solids (TS), moisture content (MC), and total organic carbon (TOC). The biological analyses were heterotrophic plate count (HPC), Live/Dead bacterial viability staining, and DNA analyses using quantitative polymerase chain reaction (qPCR). The concentration of AgNPs in sludge during the first stage was higher than the second stage, which was needed to be able to detect and observed the AgNPs by TEM. Also, this concentration of AgNPs was used by previous studies as a very acute dose of AgNPs (Liang et al., 2010; Sun et al., 2013). 


\subsubsection{Chemical analysis}

The chemical analysis included the initial analysis of soil and sludge samples in addition to the analyses that were carried out every $15 \mathrm{~d}$ from the start of the study to its completion. All chemical analyses were done in triplicate following Standard Methods (APHA et al., 2017). The soil was analyzed for CEC at the beginning of the experiment using the standard method employed by the Natural Resources Conservation Service (Rebecca, 2004). The analysis method involved the use of $1 \mathrm{M}$ ammonium acetate (NH4OAc) at $\mathrm{pH} 7$ (neutral NH4OAc). Soil samples were prepared for $\mathrm{pH}$ analysis by first suspending $5 \mathrm{~g}$ of soil in 5 $\mathrm{mL}$ of water $(1 \mathrm{~g}: 1 \mathrm{~mL}$ ratio) followed by a $30 \mathrm{~s}$ vortex of the suspension. The $\mathrm{pH}$ was measured using the Orion Star TM A326 pH/DO portable meter. The percentages of TS and $\mathrm{MC}$ were determined by drying $5 \mathrm{~g}$ of the soil sample in an oven at $105^{\circ} \mathrm{C}$ for $24 \mathrm{~h}$. TS\% represented the sample weight after drying divided by sample weight before drying and multiplying by $100 . \mathrm{MC}$ was calculated by taking the difference in the sample weight before and after drying divided by the weight of the dried sample and multiplying by $100 \%$. MC was monitored every 2-3 days and maintained at $45 \%$ by addition of sterile distilled water. TOC analysis was conducted using TOC-VCPH/CPN and TOC-control V software. Each TOC measurement run included one blank and a set of standard solution concentrations that were used to construct a calibration curve. Three replicates were taken for each TOC measurement. Soil samples were prepared by suspending $1 \mathrm{~g}$ of soil in 10 $\mathrm{mL}$ of $\mathrm{CaCl}_{2}$ solution $(1: 10 \mathrm{w} / \mathrm{v}, 0.01 \mathrm{M})$. The suspension was put in a shaker for $2 \mathrm{~h}$ and centrifuged at $1,800 \times \mathrm{g}$ for $12 \mathrm{~min}$. The supernatant was then used for the TOC analysis; these results represent soluble TOC. 


\subsubsection{Microbiological analyses}

\subsubsection{Heterotrophic bacteria}

Viability of heterotrophic bacteria in the soil samples was investigated every 15 days using the spread plate method. Tryptic soy agar (TSA) was used as the growth medium. TSA was prepared by adding $30 \mathrm{~g}$ of the powdered medium to $1 \mathrm{~L}$ of distilled water, dissolved by boiling and autoclave sterilized. After the TSA had cooled to approximately $50^{\circ} \mathrm{C}$, a volume of $22 \mathrm{~mL}$ was then poured into each Petri plate and stored at $4^{\circ} \mathrm{C}$ for a maximum of one week. Soil samples were prepared by suspending $0.1 \mathrm{~g}$ of the soil sample in $0.9 \mathrm{~mL}$ PBS (with $0.1 \%$ Tween 80 ) and vortexed for 30 s. Serial dilutions were made, and three replicates of $0.1 \mathrm{~mL}$ of $10 \mathrm{E}-04,10 \mathrm{E}-05$, and $10 \mathrm{E}-06$ dilutions were inoculated onto the TSA plates and incubated in the dark at $34 \pm 1{ }^{\circ} \mathrm{C}$. Colony forming units (CFU) were calculated after a $24 \mathrm{~h}$ incubation time.

\subsubsection{Live/Dead bacterial viability staining assay}

The distribution of Live/Dead bacterial cells in the soil reactors was investigated by staining with fluorescent dyes and observed with the Nikon Eclipse Ti-E Microscope. The soil samples were stained with BacLight staining reagent kit that was prepared by dissolving SYTO 9 stain and propidium iodide stain in $5 \mathrm{~mL}$ sterilized deionized water. The soil samples were prepared for Live/Dead analysis through suspending $0.1 \mathrm{~g}$ of soil in a $0.9 \mathrm{~mL}$ of PBS solution with $0.1 \%$ Tween 80 . The mixture, then, was vortexed for $30 \mathrm{~s}$ at high speed and, after, the mixture settled for $30 \mathrm{~min}$. A known volume of the supernatant was mixed with an equal amount of the staining reagent, and the mixture was incubated in the dark for $15 \mathrm{~min}$. Then, a $17 \mu \mathrm{L}$ of the mixture was applied to a standard microscope 
slide and cover with a $22 \mathrm{~mm}$ square coverslip. The samples were then ready for observation under the fluorescence microscope, where the ratio of Live/Dead bacterial cells could be quantified. Three tests were done for each soil sample, and three random fields of views (FOV) were chosen on each slide where images were taken. Three images were taken

at each FOV using 3 UV filters, GFP-1, Cy-3 and GFPHQ to capture live, dead and a combination of live and dead microbial cells respectively. The three sets of images were assessed, and the most representative was chosen to report, and the live cell percentage was estimated by dividing the number of live cells by the total number of cells in the image.

\subsubsection{Bacterial population analysis}

\subsection{Bacterial phyla}

The five most prevalent bacterial phyla found in soil and sludge were selected for investigating Acidobacteria, Actinobacteria, Bacteroidetes, Firmicutes, and Proteobacteria. Each soil reactor was analyzed for the presence and relative population of each phylum every $15 \mathrm{~d}$ for 105 days experiment period. Three replicate DNA extractions were done for each soil reactor using the PowerSoil DNA Isolation Kit from MO BIO Laboratories Inc. (QIAGEN Online Shop). The concentration of extracted DNA was measured using the Qubit ${ }^{\circledR}$ 2.0 Fluorometer with the dsDNA HS Assay Kits (Molecular Probes, Life technology). The extracted DNA was then used as template DNA for the phylum specific qPCR protocols. Each phylum was amplified individually using phylum specific qPCR primer sets. 


\subsection{Primers}

Primers were purchased from Life Technologies (Burlington, ON). The primers were selected based on recently published studies. Primers references and properties are shown in Table 7-4.

Table 7-4 Phyla primer pairs used in qPCR assay.

\begin{tabular}{|c|c|c|c|c|c|}
\hline Phylum & $\begin{array}{l}\text { Amplicon } \\
\text { size (bp) }\end{array}$ & $\begin{array}{l}\text { Primer } \\
\text { name }\end{array}$ & Primer sequence $\left(5^{\prime}->3^{\prime}\right)$ & $\begin{array}{l}\text { Annealing } \\
\text { temperature } \\
\left({ }^{\circ} \mathrm{C}\right) \\
\end{array}$ & References \\
\hline \multirow{2}{*}{ Acidobacteria } & \multirow{2}{*}{500} & fAcid31 & GATCCTGGCTCAGAATC & \multirow{2}{*}{55.9} & \multirow{2}{*}{$\begin{array}{l}\text { (Fierer et al., } \\
2005 \text { ) }\end{array}$} \\
\hline & & rEub518 & ATTACCGCGGCTGCTGG & & \\
\hline \multirow{2}{*}{ Actinobacteria } & \multirow{2}{*}{166} & fActi & GRDACYGCCGGGGTYAACT & \multirow{2}{*}{57.2} & \multirow{2}{*}{$\begin{array}{l}\text { (Pfeiffer et al., } \\
\text { 2014) }\end{array}$} \\
\hline & & rActi & TCWGCGATTACTAGCGAC & & \\
\hline \multirow{2}{*}{ Bacteroidetes } & \multirow{2}{*}{181} & fBdet & GCACGGGTGMGTAACRCGTACCCT & \multirow{2}{*}{61} & \multirow{2}{*}{$\begin{array}{l}\text { (Pfeiffer et al., } \\
\text { 2014) }\end{array}$} \\
\hline & & rBdet & GTRTCTCAGTDCCARTGTGGG & & \\
\hline \multirow{2}{*}{ Firmicutes } & \multirow{2}{*}{156} & fFirm & CAGCAGTAGGGAATCTTC & \multirow{2}{*}{55.3} & \multirow{2}{*}{$\begin{array}{l}\text { (Pfeiffer et al., } \\
\text { 2014) }\end{array}$} \\
\hline & & rFirm & ACCTACGTATTACCGCGG & & \\
\hline \multirow{2}{*}{ Proteobacteria } & \multirow{2}{*}{140} & $767 \mathrm{fProt}$ & AAGCGTGGGGAGCAAACA & \multirow{2}{*}{54.8} & \multirow{2}{*}{$\begin{array}{l}\text { (Morales \& } \\
\text { Holben, 2009) }\end{array}$} \\
\hline & & 907rProt & CCGTCAATTCMTTTRAGTTT & & \\
\hline \multirow{2}{*}{$\begin{array}{l}16 \mathrm{~S} \\
\text { (any bacteria) }\end{array}$} & \multirow{2}{*}{180} & $338 \mathrm{~F}$ & ACT CCT ACG GGA GGC AGC AG & \multirow{2}{*}{61.9} & \multirow{2}{*}{$\begin{array}{l}\text { (Shakya et al., } \\
\text { 2013) }\end{array}$} \\
\hline & & $518 \mathrm{R}$ & ATT ACC GCG GCT GCT GG & & \\
\hline
\end{tabular}

\subsubsection{3 qPCR protocols and annealing temperature optimization}

Phylum analysis was done using individual qPCR protocols for each sludge/soil mixture every $15 \mathrm{~d}$ over a 105 days experiment period. In each qPCR run, there were three replicate qPCR reactions per each genomic DNA sample for each phylum with three non-template controls (NTC) in each run. Reactions were performed using CFX96 Touch Real-Time PCR Detection System (BioRad) and analyzed with the CFX Manager Software. The qPCR protocol was the same for all phyla except for the annealing temperature, which was different for each phylum. The qPCR protocol consisted of $95^{\circ} \mathrm{C}$ for 5 min as an initial denaturation step, followed by 40 cycles of a $95^{\circ} \mathrm{C}$ denaturation for $10 \mathrm{~s}$, annealing for 20 $\mathrm{s}$ (Table 7-4), and an extension of $72^{\circ} \mathrm{C}$ for $15 \mathrm{~s}$, A melt curve analysis from 65 to $95^{\circ} \mathrm{C}$ 
with a $0.5^{\circ} \mathrm{C}$ increase every $5 \mathrm{~s}$. Every qPCR reaction consisted of $12.5 \mu \mathrm{L}$ qPCR buffer, $0.5 \mu \mathrm{L}(200 \mathrm{nM})$ forward primer, $0.5 \mu \mathrm{L}(200 \mathrm{nM})$ reverse primer, DNA template equilibrated to $30 \mathrm{ng}$ concentration, and water to $25 \mu \mathrm{L}$ as the final reaction volume. The qPCR reaction/buffer solution was SsoFast EvaGreen Supermix (purchased from Bio-Rad Laboratories Ltd, Canada) and contains dNTPs, Sso7d fusion polymerase, $\mathrm{MgCl}_{2}$, EvaGreen dye, and stabilizers. Preliminary experiments were done to optimize the annealing temperature for each phylum protocol. The annealing temperature that maximized amplification was determined with extracted soil DNA template in a thermal gradient $\mathrm{qPCR}$ reaction with a different annealing temperature range for each phylum.

\subsubsection{4 qPCR results analysis}

Universal 16S ribosomal RNA primers were used to amplify an $\sim 180$ bp sequence which was used to calculate the baseline $16 \mathrm{~S}$ gene copy number which was compared to each of the five phyla specific calculated gene copy numbers. The data generated relative population trends for each phylum over time. The data analysis was based on three main fundamental assumptions:

1. Cq is equivalent to $3.33 \log 10$ (target amplicon concentration).

Many studies have used a control curve to relate the number (concentration) of DNA in the qPCR product to the corresponding Cq. Svec et al. (2015) have mentioned that constructing a control curve with $\log 10$ of DNA number versus Cq (resulted from the qPCR run of serial concentrations of DNA) will be linear with a slope of 3.33 when the qPCR efficiency is $100 \%$ (Svec et al., 2015). 
2. Every bacterium has $1 \sim$ fg of DNA.

The concentration of the DNA template used in the $\mathrm{qPCR}$ reaction was $30 \mathrm{ng}$. The $\mathrm{qPCR}$ analysis in this study relies on a relative estimation of the bacterial population. If it is assumed that every bacterium has $1 \sim$ fg of DNA, then $30 \mathrm{ng}$ of DNA equal to $3 \times 10 \mathrm{E}+07$ bacteria. This is just a relative estimation because the soil extracted DNA belongs to many other living things besides bacteria such as plants and eukaryotes.

3. Every phylum has a specific copy number of $16 \mathrm{~S}$ rRNA.

Based on a study by Větrovský \& Baldrian (2013), each phylum has a specific copy number of 16S rRNA as illustrated in Table 7-5.

Table 7-5 Copy number of 16S rRNA in each bacterial genome (Větrovský \& Baldrian, 2013).

\begin{tabular}{|l|l|l|}
\hline Phylum & $\mathbf{1 6 S}$ rRNA/genome & $\begin{array}{l}\text { Mean 16S } \\
\text { rRNA/genome }\end{array}$ \\
\hline Acidobacteria & 1 & 1 \\
\hline Actinobacteria & $3.3 \pm 1.7$ & 3.3 \\
\hline Bacteroidetes & $3.5 \pm 1.5$ & 3.5 \\
\hline Firmicutes & $5.8 \pm 2.8$ & 5.8 \\
\hline Proteobacteria & $3.96 \pm 1.7$ & 4.0 \\
\hline
\end{tabular}

\subsubsection{Statistical analyses}

Most results are reported as the average of three replicates \pm standard deviation. Comparisons and significant differences between different soil reactors over time (control 1 versus control 2 and control 2 versus the lime reactor) were performed using a one-way analysis of variation (ANOVA) at 95\% confidence level. Significant differences are those with a p-value $<0.05$. If the ANOVA-test indicated a significant difference, a T-test was performed between each of the two data sets. In addition, the coefficient of variance (CV) 
was estimated for each data set, and significant differences are those with a $\mathrm{CV}>0.05$ (i.e., the level of confidence $\geq 95 \%$ ).

\subsection{Results and discussion}

\subsubsection{Characterization of AgNPs}

AgNPs suspension was examined for particle size and morphology using TEM analysis after dilution of the stock solution with nanopure water; the TEM images revealed that the AgNPs suspension was extremely pure. The images were examined with EDS, which used to examine the elemental composition of a sample, and other than carbon and copper, which are the components of the supportive grid, only silver was found (Figure 7-1). During TEM imaging the AgNPs were well distributed in water, and agglomeration or aggregation of particles was not detected. This is probably due to a PVP coating that helped the particles resist clustering and deposition.
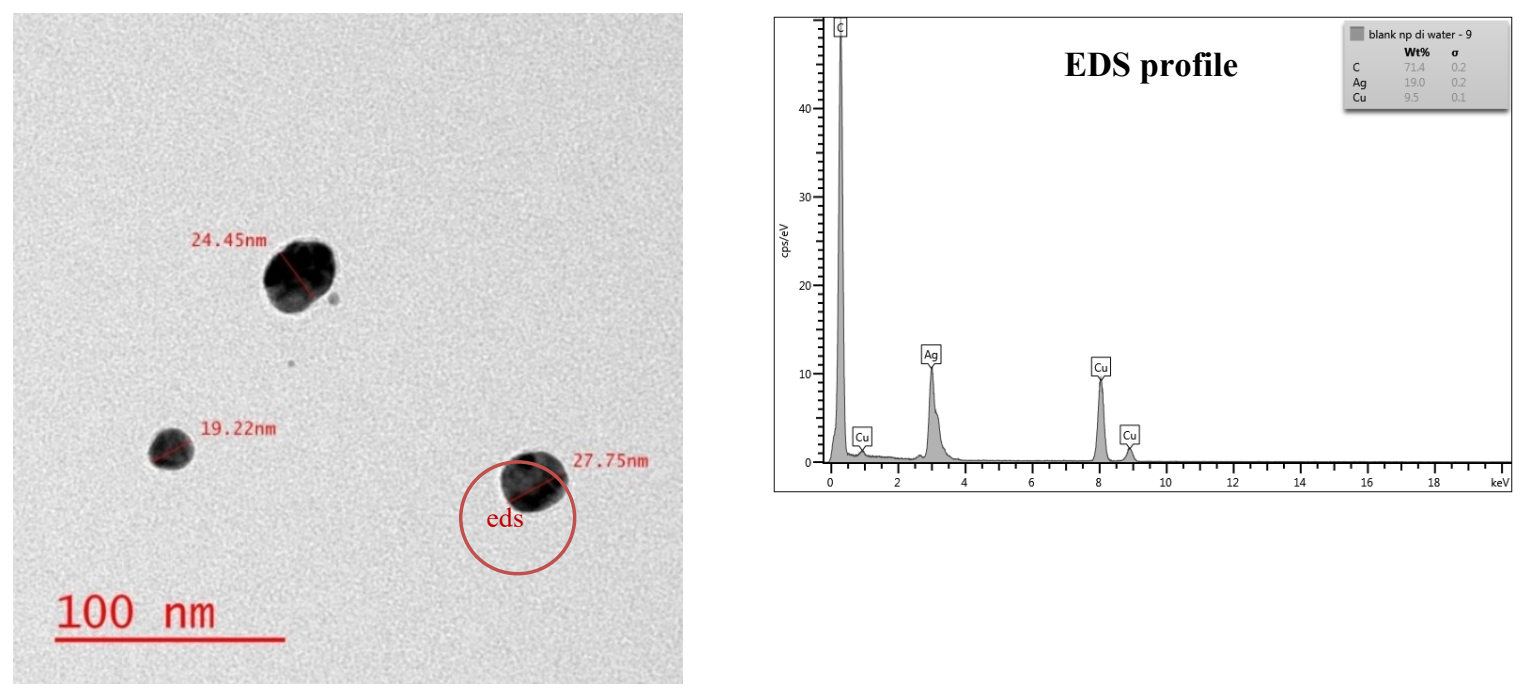

Figure 7-1 TEM image of AgNPs suspended in water with its EDS profile. 


\subsubsection{Lime stabilization process}

During the first stage of the study, AgNPs were added to the sludge at a concentration of $0.1 \mathrm{mg} \mathrm{AgNPs} / \mathrm{mL}$ sludge, and then, lime treatment was conducted, and after treatment sludge was examined for the morphological and structural changes of AgNPs using TEM. The visual observation revealed the formation of larger, less mobile particle clusters due to coagulation caused by lime particles. TEM images showed that lime removed AgNPs effectively from the aqueous phase and that AgNPs were strongly deposited to the surface of lime molecules, as shown in Figures 7-2 and 7-3. More AgNPs appeared to be attached to the surface of lime molecules in water (Figure 7-2) than in sludge (Figure 7-3). This is likely because there were more competing reactions among AgNPs, lime, and sludge constituents in sludge, and these decreased the availability of AgNPs for surface attachment. The images were, also examined with EDS, and several elements were detected, including $\mathrm{Ag}$ and $\mathrm{Ca}$ in water (Figure 7-2), and $\mathrm{Ag}, \mathrm{Ca}, \mathrm{Fe}, \mathrm{Al}$, and $\mathrm{S}$ in sludge (Figure 7-3). Lime works by raising the $\mathrm{pH}$ of the sludge and increasing its porosity. Lime also causes hydrogen sulfide to revert to sulfide and bisulfate ions, which are not volatile at elevated pH levels and hence reduce sludge odor (Turovskiy \& Mathai, 2006). Ma et al. (2013) showed that $\mathrm{Ag}_{2} \mathrm{~S}$ is the dominant silver compound found after sludge undergoes lime/heat treatment. 

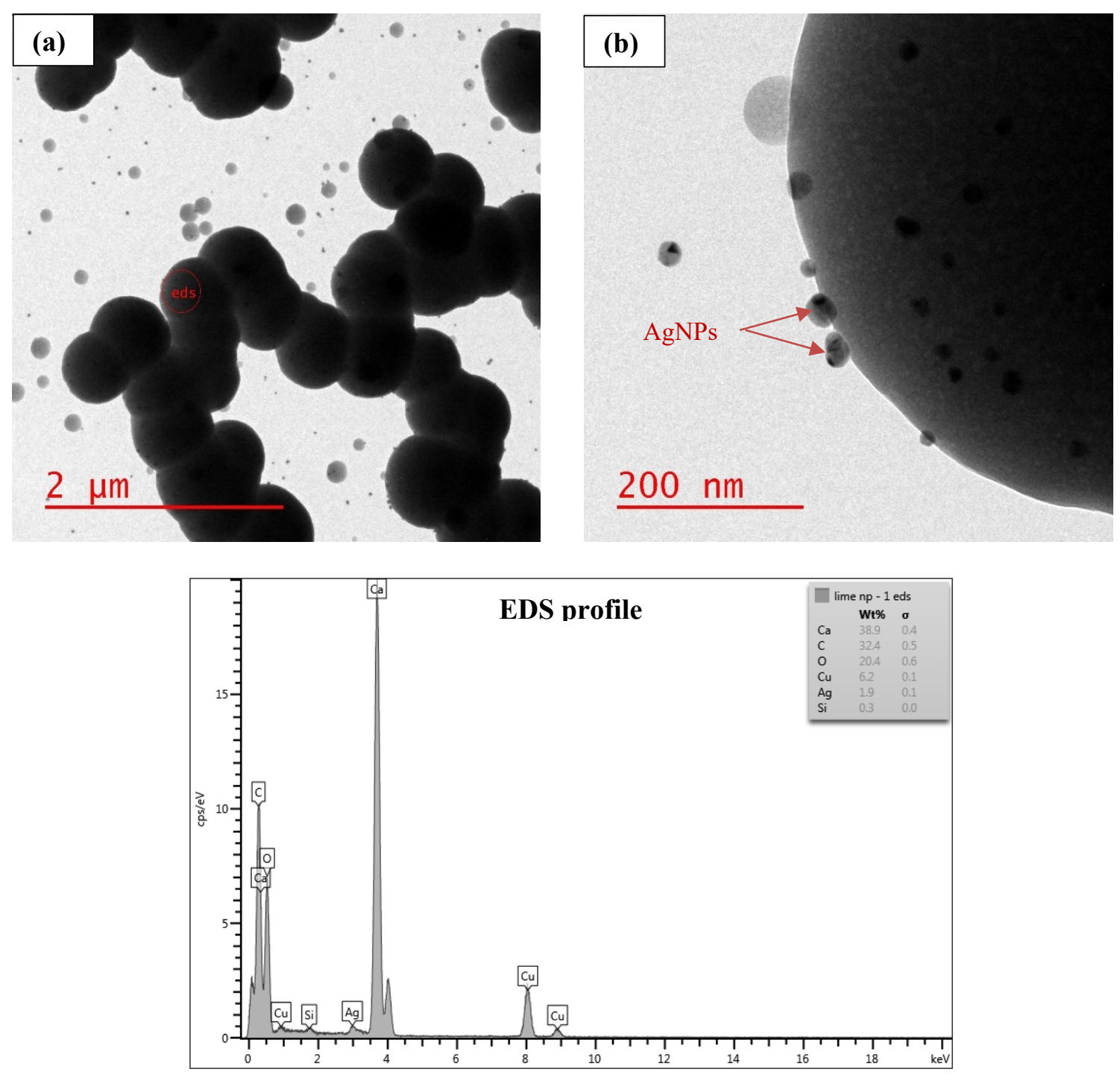

Figure 7-2 TEM images show lime treatment effect on AgNPs suspended in nanopure water, with EDS profile. (a) and (b) refer to different scales. 

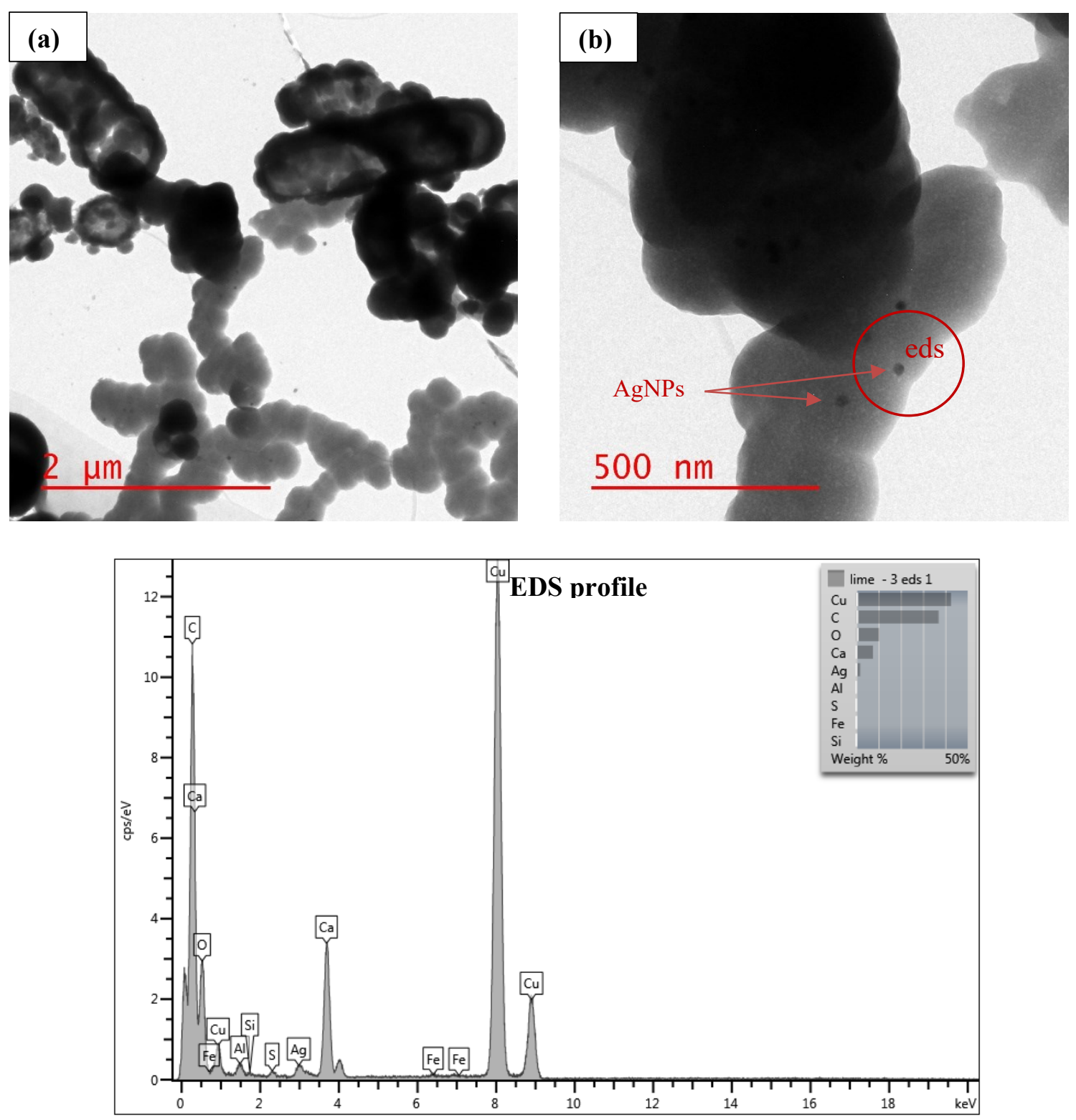

Figure 7-3 TEM images show lime treatment effect on AgNPs suspended in sludge, with EDS profile. (a) and (b) refer to different scales.

\subsubsection{Land application of lime stabilized sludge}

\subsubsection{TOC and $\mathrm{pH}$}

After land application of lime stabilized sludge, soil monitoring showed that TOC decreased over 105 days at a similar rate in all reactors, as shown in Figure 7-4(a). TOC of 
the two controls and lime soil reactors were similar, with no significant differences observed. T-test, p-values between controls 1 and 2 and between control 2 and the lime reactors were 0.96 and 0.94 , respectively. TOC for all reactors decreased significantly during the first month, with overall reduction rates of 44, 40 and $44.4 \%$ for controls 1 and 2 , and the lime reactors, respectively. Organic soil carbon plays a major role in overall soil health, as it is the food source for soil microorganisms and helps fixate soil nutrients such as nitrogen, phosphorus, and sulfur. Many factors can affect the TOC level in soil, including $\mathrm{pH}$, temperature, soil aeration, and microbial population of the soil. The observed decrease in TOC here was likely due to the acclimatization time, as well as the decomposition and consumption of organic matter by microorganisms. Microorganisms compete with each other to survive in a limited carbon environment, and no carbon source was added during the experiment.

Soil $\mathrm{pH}$, a parameter with major influence on the availability of nutrients such as nitrogen and phosphorus in the soil, is virtually essential for soil microbial content and diversity and several studies have shown the $\mathrm{pH}$ impact on AgNPs (Qu et al., 2010). The soil $\mathrm{pH}$ levels were not affected by the presence of AgNPs and decreased during the first 15 days for all soil reactors, as seen in Figure 7-4(b). This is normal as a result of acclimation to the new environment by the chemical reactions and microorganisms. The $\mathrm{pH}$ readings were stable over the remaining period, and after day 15 the $\mathrm{pH}$ control reactor dropped from neutral (an approximate $\mathrm{pH}$ value of 7) at the beginning of the experiment, to somewhat acidic at the end. The $\mathrm{pH}$ of the lime reactor was alkaline $(\mathrm{pH}=8.3)$ on day 0 and decreased to near neutral $(\mathrm{pH}=7.2)$ at the end of the experiment. Soil $\mathrm{pH}$ is an important factor when studying the behavior and impact of AgNPs. 

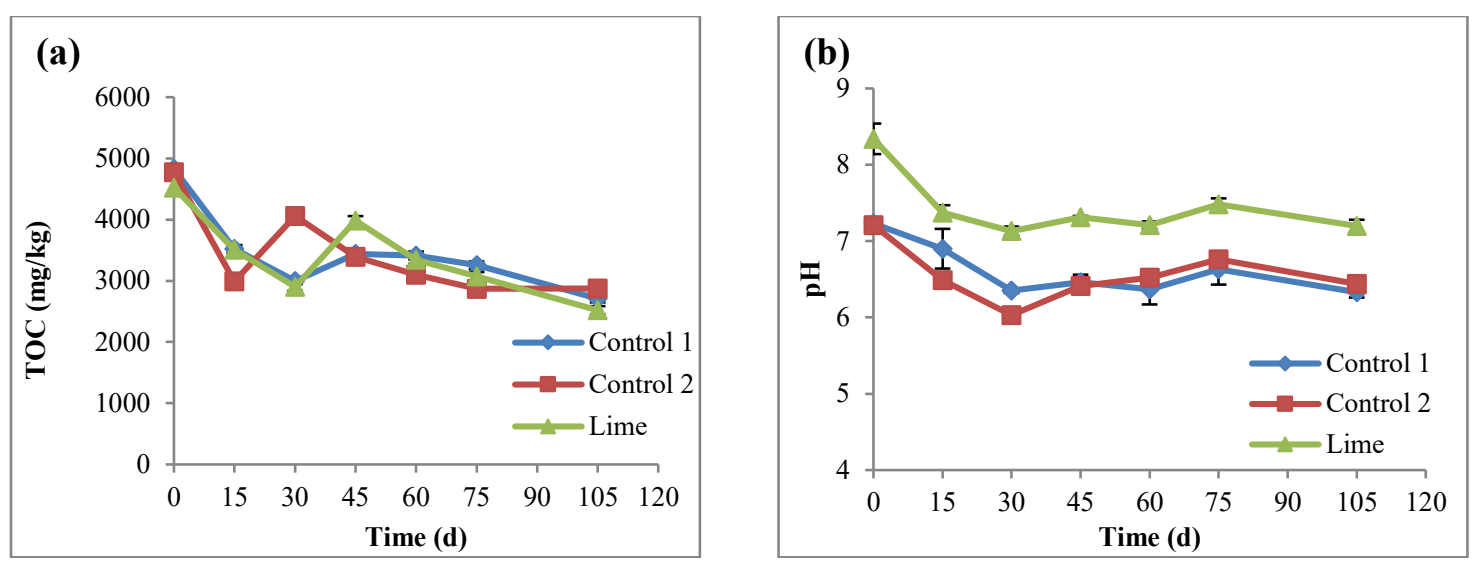

Figure 7-4 Total organic carbon concentration (a) and $\mathrm{pH}$ (b), over time. Control 1 is the soil reactor that received untreated sludge with no nanoparticles, control 2 is the soil reactor that received untreated sludge with $2 \mathrm{mg} \mathrm{AgNPs/g} \mathrm{TS} \mathrm{sludge,} \mathrm{and} \mathrm{lime} \mathrm{is} \mathrm{the} \mathrm{soil} \mathrm{reactor} \mathrm{that}$ received lime treated sludge with $2 \mathrm{mg}$ AgNPs/g TS sludge.

\subsubsection{Impact of AgNPs on heterotrophic bacteria and Live/Dead viability}

Under lime treatment, heterotrophic bacteria showed slightly different trends over time compared to controls 1 and 2. The CFU were also very similar, suggesting that there were no significant impacts due to the presence of AgNPs. On day 0, as seen in Figure 7-5(a), HPC for a lime soil reactor was lower by $\sim 1 \mathrm{Log} 10 \mathrm{CFU} / \mathrm{mL}$, most likely due to the effect of high $\mathrm{pH}$. However, CFU under lime treatment showed the highest increase over time (16.6\% increase), compared to controls $1,(11.4 \%$ decrease) and 2 (9.8\% decrease), as shown in Figure 7-5(a). This is likely due to the solubilization of substrates after lime treatment, which makes them readily available for microorganisms. T-test showed no significant difference between controls 1 and $2(\mathrm{p}$-value $\approx 0.58)$. Thus, the presence of AgNPs at this concentration had no significant effect on the heterotrophic bacteria in the soil. This finding is supported by other studies that showed no effect of AgNPs on heterotrophic bacteria (Liang et al., 2010), even though it was reported that lime seemed to enhance CFU. HPC results showed significant differences in the number of heterotrophic 
colonies between control 2 and the lime reactor ( $\mathrm{p}$-value $\approx 0.02$ ), which indicates that lime treatment of sludge significantly impacted the heterotrophic bacteria in the soil.

In addition to the enumeration of the heterotrophic bacteria, Live/Dead viability staining was also evaluated using the 2 separate fluorescent dyes, CYTO 9 and propidium iodide. Bacterial cells with intact membranes are stained fluorescent green, while those with damaged membranes are stained fluorescent red. The percentage of live cells for both control reactors fluctuated over time, and they followed similar trends (increasing mode), and ANOVA analysis showed no significant differences between the controls. However, as shown in Figure 7-5(b) there were small increases in the percentage of live cells in all the soil reactors, indicating that neither the AgNPs nor the sludge lime treatment caused significant toxicity to soil bacteria at the concentrations applied. The bacterial Live/Dead staining images are presented in the supplementary data (Figures S-2 to S-4).
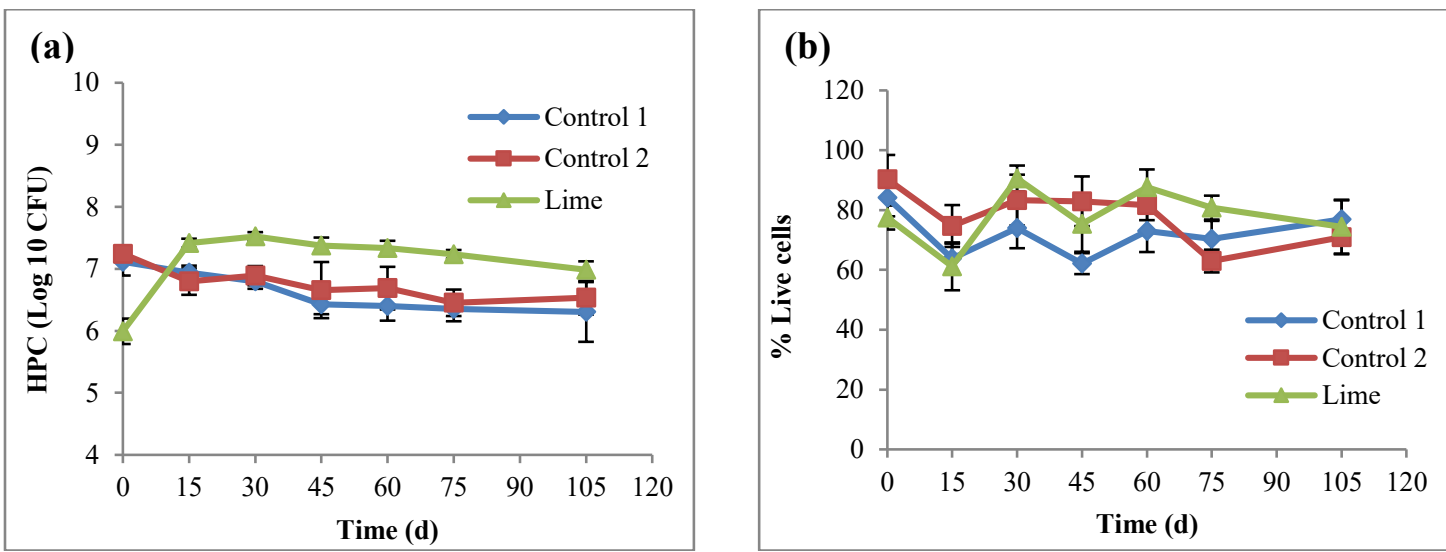

Figure 7-5 HPC, represented by CFU (a) and the percentage of live cells in soil reactors (b) over time. Control 1 is the soil reactor that received untreated sludge with no nanoparticles, control 2 is the soil reactor that received untreated sludge with 2 mg AgNPs/g TS sludge, and lime is the soil reactor that received lime treated sludge with 2 mg AgNPs/g TS sludge. 


\subsubsection{Impact of AgNPs on selected soil microorganisms}

After extraction of the genomic DNA from the soil reactors (Figure S-1), it was analyzed for the presence and concentration of five selected bacterial phyla using qPCR. The $\mathrm{Cq}$ (quantitation cycle) values from the qPCR results were converted to calculated $\mathrm{CFU}$ equivalents (CCE), based on assumptions discussed in the material and methods, Section 7.2.7.3.4. CCE was used to estimate the mean abundance of each phylum in all reactors. Of the five phyla, Acidobacteria was the most highly abundant in all the soil reactors, with $29 \%$ relative abundance, Proteobacteria was the next, with $26 \%$ abundance, and Bacteroidetes had 3\% relative abundance, which was the least, while Firmicutes and Actinobacteria, both, had $21 \%$ relative abundance, and, thus, were similar in terms of having medium abundance compared to the other assessed phyla. These findings correspond with those of other researchers who studied the soil bacterial diversity and abundance (Fierer et al., 2005; Rousk et al., 2010).

Similar to what was observed in our previous study (Chapter 6), all the studied phyla showed similar $\mathrm{Cq}$ values in controls 1 and 2 throughout the experiment with very little variance in their CCEs. T-test p-values were 0.04, 0.6, 0.52, 0.43 and 0.84 for Proteobacteria, Acidobacteria, Actinobacteria, Bacteroidetes and Firmicutes, respectively. The phyla relative abundance for the controls throughout the experiment were very similar with variations between them of less than one Log10 CCE. Thus, AgNPs at a concentration of $20 \mathrm{mg} \mathrm{AgNPs} / \mathrm{g}$ soil had minimal impact on the diversity and presence of these five phyla in soil. 
Assessment of phyla abundance in the soil reactors that received lime treated sludge showed a higher degree of variation in Cq values (leading to dissimilar CCE profiles) compared to the controls (Figure 7-6). Under the lime treatment, all studied phyla showed an increase in abundance except Bacteroidetes.

The CCE trends over time for Acidobacteria were similar in control 2 and lime reactors, and ANOVA testing showed no major differences in the CCE of Acidobacteria in all soil reactors ( $\mathrm{p}$-value $>0.05$ ). This indicates that Acidobacteria is not adversely affected by the presence of AgNPs or the sludge lime treatment; CCE increases of 10, 6 and 5\% for control 1, control 2 and lime soil reactors, respectively. This is likely due to the potential of Acidobacteria to tolerate various pollutants and heavy metals (Gremion et al., 2003; Paul et al., 2006; Sánchez-Peinado et al., 2010). Acidobacteria is also responsible for several beneficial soil fertility functions, including nitrate and nitrite reduction (Ward et al., 2009).

Actinobacteria also showed similar CCE trends over time for control 2 and lime reactors, as seen in Figure 7-6. Analysis with ANOVA showed no major differences ( $p$-value $=0.95$ ) between control 2 and the lime reactor in relative CCE trends. However, lime treatments seemed to promote Actinobacteria abundance with a 9\% increase in CCE throughout the experiment. Actinobacteria is one of the most abundant and important bacterial phyla in soil, and it helps with carbon degradation including cellulose, $\mathrm{CO}_{2}$ fixation, nitrogen fixation, and phosphorus uptake ( $\mathrm{Li}$ et al., 2014). Thus, Actinobacteria is an important factor to consider when studying the impact of nanoparticles on the soil. The relative CCE of Bacteroidetes also showed no significant differences between control 2 and the lime reactors, and ANOVA results showed a p-value of 0.16. However, Bacteroidetes CCE was 
virtually unchanged in controls 1 and 2, and it decreased by $6 \%$ under the lime treatment. Bacteroidetes has several important roles in soil, including solubilization of minerals such as phosphate, production of siderophores, and synthesis of growth-stimulating phytohormones (Soltani et al., 2010). The results of the relative CCE of Firmicutes showed a significant difference between control 2 and the lime reactor ( $\mathrm{p}$-value $=0.0034)$, although, this was due to the variation in the $\mathrm{CCE}$ at the start of the experiment, where the lime soil reactor had a higher CCE value, likely due to the effect of lime.

Firmicutes is an important soil bacterial phylum as it includes a number of genera, such as Bacillus and Paenibacillus that are key to soil fertility, including atmospheric nitrogenfixation, solubilization of minerals, suppression of plant pathogens, production of siderophores, synthesis of growth-stimulating phytohormones and bioremediation (Jeong et al., 2012; Grady et al., 2016; Radhakrishnan et al., 2017). Thus, the results showed no significant impact of AgNPs on the abundance of Firmicutes, but sludge lime treatment enhanced their abundance.

The trends over time of Proteobacteria CCE exhibited no major differences between control 2 and the lime reactor ( $\mathrm{p}$-value $=0.5$ ). Proteobacteria phylum is common in soil and it plays an important role in soil fertility functions, including carbon degradation such as aromatic compounds, $\mathrm{CO}_{2}$ fixation, nitrogen fixation, oxidation of iron, sulfur, and methane, phosphorus uptake and suppression of plant pathogens (Li et al., 2014; Itävaara et al., 2016). Proteobacteria assessment is essential when assessing the toxicity risk of nanoparticles. Thus, the result showed no significant impact of AgNPs at $20 \mathrm{mg} / \mathrm{kg}$ soil, or the sludge lime treatment on the abundance of Proteobacteria in the soil. 

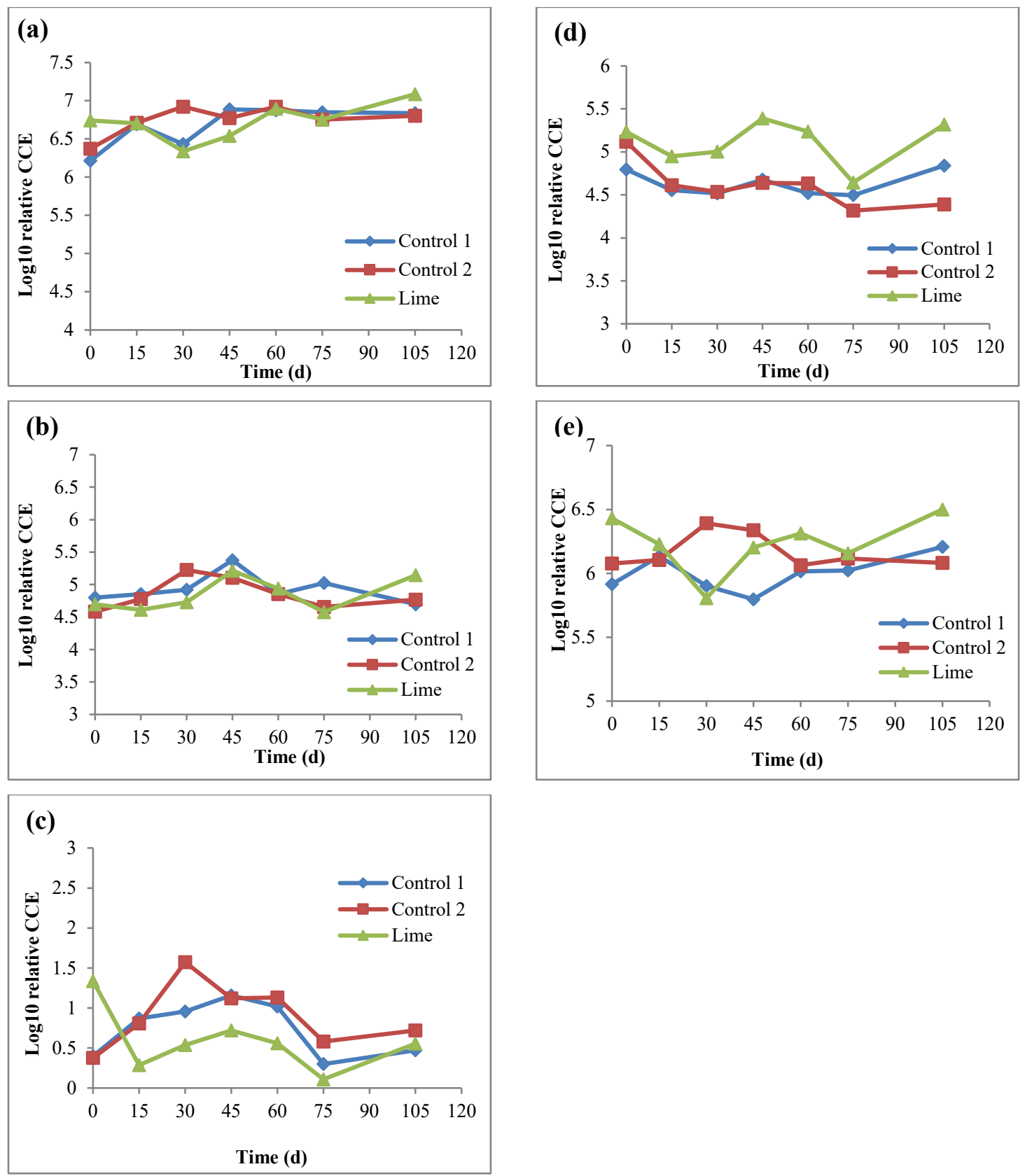

Figure 7-6 Relative change in CCE for Acidobacteria (a), Actinobacteria (b), Bacteroidetes (c), Firmicutes (d) and Proteobacteria (e). Control 1 is the soil reactor that received untreated sludge with no nanoparticles, control 2 is the soil reactor that received untreated sludge with $2 \mathrm{mg} \mathrm{AgNPs/g} \mathrm{TS} \mathrm{sludge,} \mathrm{and} \mathrm{lime} \mathrm{is} \mathrm{the} \mathrm{soil} \mathrm{reactor} \mathrm{that} \mathrm{received} \mathrm{lime} \mathrm{treated} \mathrm{sludge} \mathrm{with} 2$ mg AgNPs/g TS sludge. 
The stability and transformation of AgNPs after release into the environment could depend on several factors, including the complexity of the media, the forms of the nanoparticles, surface chemistry, concentration and exposure time; thus, these play important roles in the toxicity of AgNPs. In this study, interaction with sludge constituents or sludge lime stabilization impacted the physical and chemical characteristics of AgNPs, as was evident in the results. For example, organic matter present in sludge and soil could surface coat AgNPs, thereby stabilizing them and reducing their aggregation and toxicity (Dinesh et al., 2012; Kramer et al., 2002) and also, considering sludge treatment before the land application is important in the case of lime stabilization. Studies have shown that lime treatment affects the chemical and physical characteristics of sludge through several reactions, such as hydrolysis, saponification, and acid neutralization, which can affect the surface chemistry of AgNPs and alter their behavior and interaction with their surroundings. Soil bacteria are the microbial community responsible for several soil ecosystem functions, including recycling of organic matter and nutrients, degradation of toxins such as heavy metals, and suppression of pathogens (Emmerling et al., 2002; Aislabie \& Deslippe, 2013; Gupta et al., 2015). Several studies have shown negative results of AgNPs on soil microbial activities (Grün et al., 2019) and, thus, it is very critical to examine these results.

This paper studied the impact of AgNPs on population and diversity of five major bacterial phyla in soil. The five phyla: Acidobacteria, Actinobacteria, Bacteroidetes, Firmicutes, and Proteobacteria, were selected as they are the most abundant group of phyla in soil and play a very important role in beneficial soil functions. Overall, the study found no significant variations between controls 1 and 2 for the five assessed phyla, and this was supported by 
other factors such as HPC and the percentage of live cells which were also not significantly different between the controls. This indicates that AgNPs at a concentration of $20 \mathrm{mg}$ AgNPs $/ \mathrm{kg}$ soil showed no significant impact on soil biological activities, in terms of the assessed elements. This could be due to many factors, such as the concentration of AgNPs, that is too low to exhibit toxicity. Another factor is the interaction of AgNPs with the sludge that results in various physicochemical transformations before reaching the soil. This can alter AgNPs behavior and affect bioavailability and toxicity, such as reduction, oxidation, aggregation, and dissolution (Park et al., 2013). For example, AgNPs interact strongly with sulfur and produce silver sulfide $\left(\mathrm{Ag}_{2} \mathrm{~S}\right)$ when they are discharged into the sewer system and during wastewater treatment (Kim et al., 2010; Kaegi et al., 2013). The interaction of AgNPs with sulfur and the subsequent production of silver sulfide results in a significant reduction in the toxicity of AgNPs due to the lower solubility of silver sulfide and limits potential short-term environmental impacts (Levard et al., 2012). A study by Ma et al. (2013) showed that $\mathrm{Ag}_{2} \mathrm{~S}$ is the dominant silver compound found after lime/heat treatment of sludge. However, the soil reactor that received lime treated sludge behaved differently than the controls, either due to the lime impacting the soil directly, or the effect of AgNPs modified by the lime treatment of the sludge. In general, sludge lime treatment enhanced the relative abundance of the assessed phyla except for Bacteroidetes, which is likely due to the lime solubilizing nutrients and organic carbon, though this was not clear from the TOC concentration of the soil over time (Figure 7-4(a)).

\subsection{Conclusions}

This study investigated how lime stabilization affects the transformation of AgNPs in sludge and its impact on the soil bacterial activity after land application of the lime 
stabilized sludge. Based on the experimental results, the following conclusions were drawn:

1. Visual observation of TEM images and associated EDS demonstrated how lime treatment can effectively remove AgNPs from the liquid phase and concentrate them in sludge solids through the association of AgNPs to the lime molecules.

2. The results indicated that presence of AgNPs (at $2 \mathrm{mg} \mathrm{AgNPs} / \mathrm{g}$ TS of sludge) or lime stabilization of sludge did not have a significant impact on the soil TOC, HPC, and the percentage of the live cells.

3. Metagenomic analysis showed that Acidobacteria was the most abundant phylum and Bacteroidetes was the least abundant among Proteobacteria, Acidobacteria, Actinobacteria, and Firmicutes in all the soil reactors.

4. The results and statistical analyses showed that the presence of AgNPs at $2 \mathrm{mg}$ AgNPs/g TS of sludge (which corresponds to $20 \mathrm{mg} \mathrm{AgNPs/kg}$ soil) did not have a significant impact on the population of the assessed phyla (Acidobacteria, Actinobacteria, Bacteroidetes, Firmicute, and Proteobacteria). This was evident by the minimal differences in the relative CCE of each phylum between control 1 (no AgNPs) and control 2 (AgNPs).

5. The study also found that sludge lime stabilization did not have a significant impact on the average relative abundance of the five assessed phyla in soil.

This study produced critical data to better understand the impact of lime treated sludge with AgNPs on soil bacteria, and their critical roles in soil fertility functions. 


\section{References}

Aislabie, J., \& Deslippe, J. R. (2013). Soil microbes and their contribution to soil services. In J. Dymond (Ed.), Ecosystem services in New Zealand-conditions and trends (pp. 143-161). Lincoln, New Zealand: Manaaki Whenua Press.

APHA, A. P., American water works Association, A., \& Water environment federation, W. (2017). Standard methods for the examination of water and wastewater (23 ed.). (E. Rice, R. Baird, \& A. Eaton, Eds.) Washington, DC, USA: APHA, American Public Health Association; American water works Association, AWWA; Water environment federation, WEF.

Benn, T. M., \& Westerhoff, P. (2008). Nanoparticle silver released into water from commercially available sock fabrics. Environmental Science \& Technology, 42(11), 4133-4139.

Blaser, S. A., Martin, S., Matthew, M., \& Konrad, H. (2008). Estimation of cumulative aquatic exposure and risk due to silver: contribution of nano-functionalized plastics and textiles. Science of The Total Environment, 390(2), 396-409.

CCME, A. (2010). Review of the Current Canadian Legislative Framework for Wastewater Biosolids. Canadian Council of Ministers of the Environment (CCMC).

City of Ottawa, C. o. (2014). City of Ottawa annual report. Ottawa, Canada: Citty of Ottawa.

Clint, W., Chen, W.-Y., Shammas, N. K., \& Wang, L. K. (2007). Lime Stabilization. In N. K. Shammas, \& L. K. Wang (Eds.), Handbook of Environmental Engineering: Biosolids Treatment Processes (pp. 207-241). Totowa, N: The Humana Press Inc.

Colman, B. P., Arnaout, C. L., Anciaux, S., Gunsch, C. K., Hochella Jr, M. F., Kim, B., . . al., e. (2013). Low Concentrations of Silver Nanoparticles in Biosolids Cause Adverse Ecosystem Responses under Realistic Field Scenario. PLOS One, 8(2), e57189.

Dimkpa, C. O., McLean, J. E., Britt, D. W., \& Anderson, A. J. (2012). Bioactivity and biomodification of $\mathrm{Ag}, \mathrm{ZnO}$, and $\mathrm{CuO}$ nanoparticles with relevance to plant performance in agriculture. Industrial Biotechnology, 8(6), 344-357.

Dinesh, R., Anandaraj, M., Srinivasan, V., \& Hamza, S. (2012). Engineered nanoparticles in the soil and their potential implications to microbial activity. Geoderma, 173, 19-27. 
Emmerling, C., Michael, S., Hartmann, A., \& Kandeler, E. (2002). Functional diversity of soil organisms - a review of recent research activities in Germany. Journal of Plant Nutrition and Soil Science, 165(4), 408-420.

EPA, U. E. (2018). Standards for the use or disposal of sewage sludge. Washington, DC, US: EPA40 CFR 503.

Erland, B., Díaz-Raviña, M., Frostegård, Å., \& Campbe, C. D. (1998). Effect of metal-rich sludge amendments on the soil microbial community. Applied and Environmental Microbiology, 64(1), 238-245.

Fabrega, J., Renshaw, J. C., \& Lead, J. R. (2009). Interactions of silver nanoparticles with Pseudomonas putida biofilms. Environmental Science \& Technology, 43(23), 9004-9009.

Fierer, N., Jackson, J. A., Vilgalys, R., \& Jackson, R. B. (2005). Assessment of soil microbial community structure by use of taxon-specific quantitative PCR assays. Appl. Environ. Microbiol., 71(7), 4117-4120.

Geranio, L., Manfred, H., \& Bernd, N. (2009). The behavior of silver nanotextiles during washing. Environmental Science \& Technology, 43(21), 8113-8118.

Gottschalk, F., Tobias, S., Roland W., S., \& Bernd, N. (2009). Modeled environmental concentrations of engineered nanomaterials ( $\mathrm{TiO} 2, \mathrm{ZnO}, \mathrm{Ag}, \mathrm{CNT}$, fullerenes) for different regions. Environmental Science \& Technology, 43(24), 9216-9222.

Grady, E. N., MacDonald, J., Liu, L., Richman, A., \& Yuan, Z.-C. (2016). Current knowledge and perspectives of Paenibacillus: a review. Microbial Cell Factories, 15(1), 203.

Gremion, F., Chatzinotas, A., \& Harms, H. (2003). Comparative 16S rDNA and 16S rRNA sequence analysis indicates that Actinobacteria might be a dominant part of the metabolically active bacteria in heavy metal-contaminated bulk and rhizosphere soil. Environmental Microbiology, 5(10), 896-907.

Grün, A.-L., Manz, W., Kohl, Y. L., Meier, F., Straskraba, S., Jost, C., . . Emmerling, C. (2019). Impact of silver nanoparticles (AgNP) on soil microbial community depending on functionalization, concentration, exposure time, and soil texture. Environmental Sciences Europe, 31(1), 15. 
Gupta, G., Parihar, S. S., Ahirwar, N. K., Snehi, S. K., \& Singh, V. (2015). Plant growth promoting rhizobacteria (PGPR): current and future prospects for development of sustainable agriculture. J Microb Biochem Technol, 7(2), 096-102.

Itävaara, M., Salavirta, H., Marjamaa, K., \& Ruskeeniemi, T. (2016). Geomicrobiology and metagenomics of terrestrial deep subsurface microbiomes. Advances in Applied Microbiology, 94(Academic Press), 1-77.

Jeong, H., Jeong, D.-E., Kim, S. H., Song, G. C., Park, S.-Y., Ryu, C.-M., . . Choi, S.-K. (2012). Draft Genome sequence of the plant growth-promoting Bacterium Bacillus siamensis KCTC 13613T. Journal of Bacteriology, 194(15), 4148-4149.

Johansson, M., Stenberg, B., \& Torstensson, L. (1999). Microbiological and chemical changes in two arable soils after long-term sludge amendments. Biology and Fertility of Soils, 30(12), 160-167.

Kaegi, R., Voegelin, A., Ort, C., Sinnet, B., Thalmann, B., Krismer, J., . . Mueller, E. (2013). Fate and transformation of silver nanoparticles in urban wastewater systems. Water Research, 47(12), 3866-3877.

Kaegi, R., Voegelin, A., Sinnet, B., Zuleeg, S., Hagendorfer, H., Burkhardt, M., \& Siegrist, H. (2011). Behavior of metallic silver nanoparticles in a pilot wastewater treatment plant. Environmental Science \& Technology, 45(9), 3902-3908.

Kim, B., Park, C.-S., Murayama, M., \& Hochella Jr., M. F. (2010). Discovery and characterization of silver sulfide nanoparticles in final sewage sludge products. Environmental Science \& Technology, 44(19), 7509-7514.

Kramer, J., Benoit, G., Bowles, K., DiToro, D., Herrin, R., Luther III, G., . . Shaw, J. (2002). Environmental chemistry of. In Silver in the Environment: Transport, Fate, and Effects. (pp. 1-25). Pensacola, FL, USA: SETAC.

Levard, C., Hotze, E. M., Lowry, G. V., \& Brown Jr., G. E. (2012). Environmental transformations of silver nanoparticles: impact on stability and toxicity. Environmental Science \& Technology, 46(13), 6900-6914. 
Li, X., Rui, J., Xiong, J., Li, J., He, Z., Zhou, J., . . Mackie, R. I. (2014). Functional potential of soil microbial communities in the maize rhizosphere. PLoS One, 9(11), e112609.

Liang, Z., Das, A., \& Hu, Z. (2010). Bacterial response to a shock load of nanosilver in an activated sludge treatment system. Water Research, 44(18), 5432-5438.

Lorenz, C., Hagendorfer, H., Goetz, N. v., Kaegi, R., Gehrig, R., Ulrich, A., . . Hungerbühler, K. (2011). Nanosized aerosols from consumer sprays: experimental analysis and exposure modeling for four commercial products. Journal of Nanoparticle Research, 8(13), 33773391.

Ma, R., Levard, C., Judy, J. D., Unrine, J. M., Durenkamp, M., Martin, B., . . Lowry, G. V. (2013). Fate of zinc oxide and silver nanoparticles in a pilot wastewater treatment plant and in processed biosolids. Environmental Science \& Technology, 48(1), 104-112.

Masrahi, A., VandeVoort, A. R., \& Arai, Y. (2014). Effects of silver nanoparticle on soilnitrification processes. Archives of Environmental Contamination and Toxicology, 66(4), 504-513.

McGee, C. F., Storey, S., Clipson, N., \& Doyle, E. (2017). Soil microbial community responses to contamination with silver, aluminum oxide and silicon dioxide nanoparticles. Ecotoxicology, 26(3), 449-458.

Metcalf, L., \& Eddy, H. P. (2003). Waste Water Engineering: Treatment and Reuse (4 ed.). New York: McGraw-Hill.

Morales, S. E., \& Holben, W. E. (2009). Empirical testing of 16S rRNA gene PCR primer pairs reveals variance in target specificity and efficacy not suggested by in silico analysis. Applied and Environmental Microbiology, 75(9), 2677-2683.

Nielsen, A. H., Vollertsen, J., Jensen, H. S., Madsen, H. I., \& Hvitved-Jacobsen, T. (2006). Aerobic and anaerobic transformations of sulfide in a sewer system-field study and model simulations. Proceedings of the Water Environment Federation, 9, 3654-3670.

OME, O. M., \& OMAFRA, O. M. (1996). Guidlines for The Utilization of Biosolid and Other Waste on Agricultural lands. Ontario, Canada: Ontario. 
Paul, D., Pandey, G., Meier, C., Meer, J. R., \& Jain, R. K. (2006). Bacterial community structure of a pesticide-contaminated site and assessment of changes induced in community structure during bioremediation. FEMS Microbiology Ecology, 57(1), 116-127.

Pfeiffer, S., Milica, P., Birgit, M., Kathrin, L., Evelyn, H., Paul, L., . . Angela, S. (2014). Improved group-specific primers based on the full SILVA 16S rRNA gene reference database. Environmental Microbiology, 16(8), 2389-2407.

Qu, F., Xu, H., Wei, H., La, W., Xiong, Y., Xu, F., . . Wang, Y. A. (2010). Effects of pH and temperature on antibacterial activity of silver nanoparticles. Biomedical Engineering and Informatics (pp. 2033-2037). IEEE.

Radhakrishnan, R., Hashem, A., \& Abd_Allah, E. F. (2017). Bacillus: a biological tool for crop improvement through bio-molecular changes in adverse environments. Frontiers in Physiology, 8, 667.

Rebecca, B. (2004). Soil survey laboratory method manual. Soil Survey Laboratory Investigations Report.

Rousk, J., Bååth, E., Brookes, P. C., Lauber, C. L., Lozupone, C., Caporaso, J. G., . . Fierer, N. (2010). Soil bacterial and fungal communities across a $\mathrm{pH}$ gradient in an arable soil. The ISME Journal, 4(10), 1340.

Sánchez-Peinado, M. d., González-López, J., Martínez-Toledo, M. V., Pozo, C., \& Rodelas, B. (2010). Influence of linear alkylbenzene sulfonate (LAS) on the structure of Alphaproteobacteria, Actinobacteria, and Acidobacteria communities in a soil microcosm. Environmental Science and Pollution Research, 17(3), 779-790.

Shafer, M. M., Joel T., O., \& David E., A. (1998). Removal, partitioning, and fate of silver and other metals in wastewater treatment plants and effluent-receiving streams. Environmental Toxicology and Chemistry, 17(4), 630-641.

Shakya, M., Christopher, Q., James H., C., Zamin K., Y., Christopher W., S., \& Mircea, P. (2013). Comparative metagenomic and rRNA microbial diversity characterization using archaeal and bacterial synthetic communities. Environmental Microbiology, 15(6), 1882-1899. 
Shammas, N. K., \& Wang, L. K. (2007). Land application of biosolids. In L. K. Wang, \& N. K. Shammas (Eds.), Handbook of environmental engineering biosolids treatment processes (pp. 705-745). Totowa, New Jersey: Humana Press Inc.

Shammas, N. K., \& Wang, L. K. (2007). Land Application of Biosolids. In L. K. Wang, N. K. Shammas, \& Y.-T. Hung (Eds.), Biosolids Treatment Processes (pp. 705-745). Totowa, N.J: Humana Press, Springer.

Soltani, A.-A., Khavazi, K., Asadi-Rahmani, H., Omidvari, M., Dahaji, P. A., \& Mirhoseyni, H. (2010). Plant Growth Promoting Characteristics in Some Flavobacterium spp. Isolated from Soils of Iran. Journal of Agricultural Science, 2(4), 106-115.

SOURCE, P. O. (2019). The Project on Emerging Nanotechnologies, consumer product inventory Retrieved July 21, 2019, from https://www.nanotechproject.org/cpi/browse/nanomaterials/silver-nanoparticle/

Svec, D., Ales, T., Vendula, N., Michael W., P., \& Mikael, K. (2015). How good is a PCR efficiency estimate: Recommendations for precise and robust qPCR efficiency assessments. Biomolecular Detection and Quantification, 3, 9-16.

Turovskiy, I. S., \& Mathai, P. K. (2006). Wastewater Sludge Processing . New Jersey: John Wiley and Sons.

Větrovský, T., \& Baldrian, P. (2013). The variability of the 16S rRNA gene in bacterial genomes and its consequences for bacterial community analyses. PloS One, 8(2), e57923.

Ward, N. L., Challacombe, J. F., Janssen, P. H., Henrissat, B., Coutinho, P. M., Wu, M., . . . Barabote, R. (2009). Three genomes from the phylum Acidobacteria provide insight into the lifestyles of these microorganisms in soils. Appl. Environ. Microbiol, 75(7), 2046-2056.

Whitley, A. R., Levard, C., Oostveen, E., Bertsch, P. M., Matocha, C. J., Kammer, F. v., \& Unrine, J. M. (2013). Behavior of Ag nanoparticles in soil: effects of particle surface coating, aging and sewage sludge amendment. Environmental Pollution, 182, 141-149. 


\section{Supplementary data}

\section{Lime stabilization preliminary experiments}

Table S-1 Lime doses and corresponding $\mathrm{pH}$ levels.

\begin{tabular}{|c|c|}
\hline Lime/TS of sludge $(\mathrm{g} / \mathrm{g})$ & $\mathrm{pH}$ \\
\hline 0.1 & 10.2 \\
\hline 0.15 & 10.94 \\
\hline 0.27 & 12.08 \\
\hline 0.3 & 12.11 \\
\hline
\end{tabular}




\section{DNA concentration}

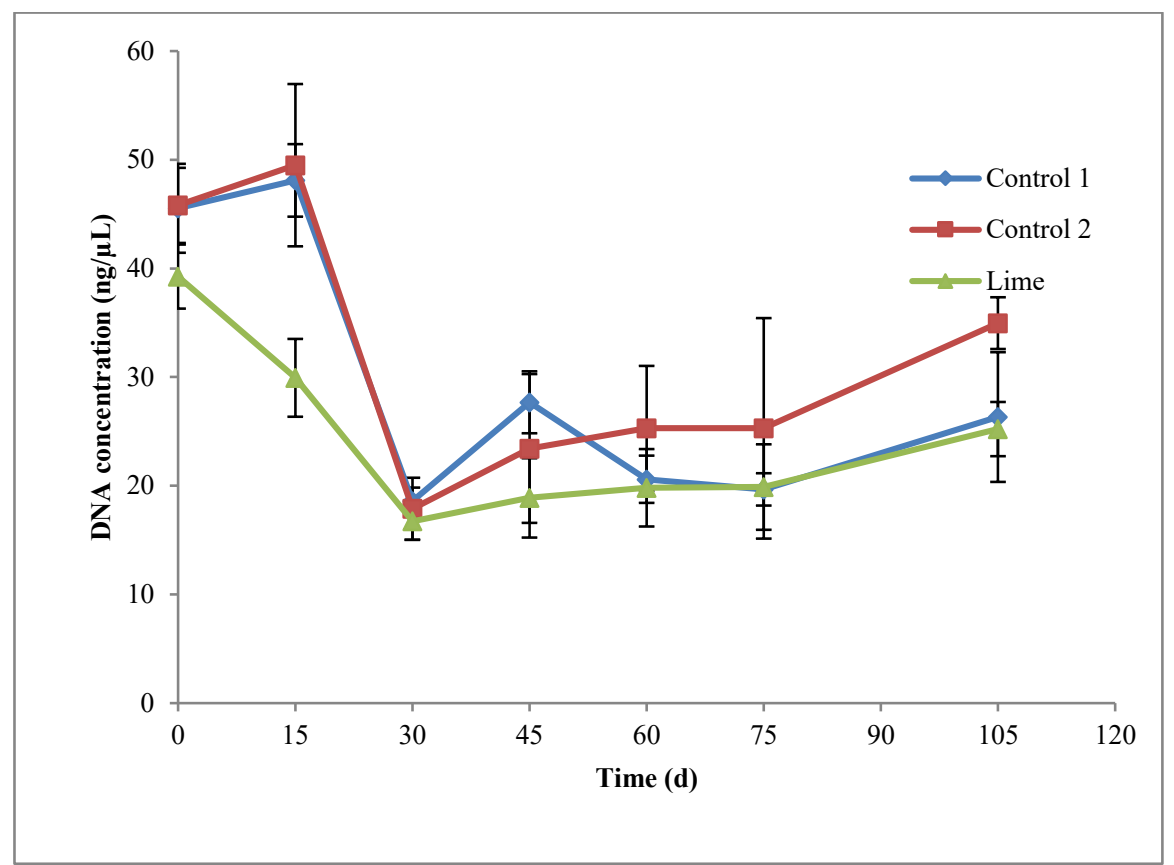

Figure S-1 DNA concentration for the controls and lime reactors over time. 


\section{Live/Dead images}
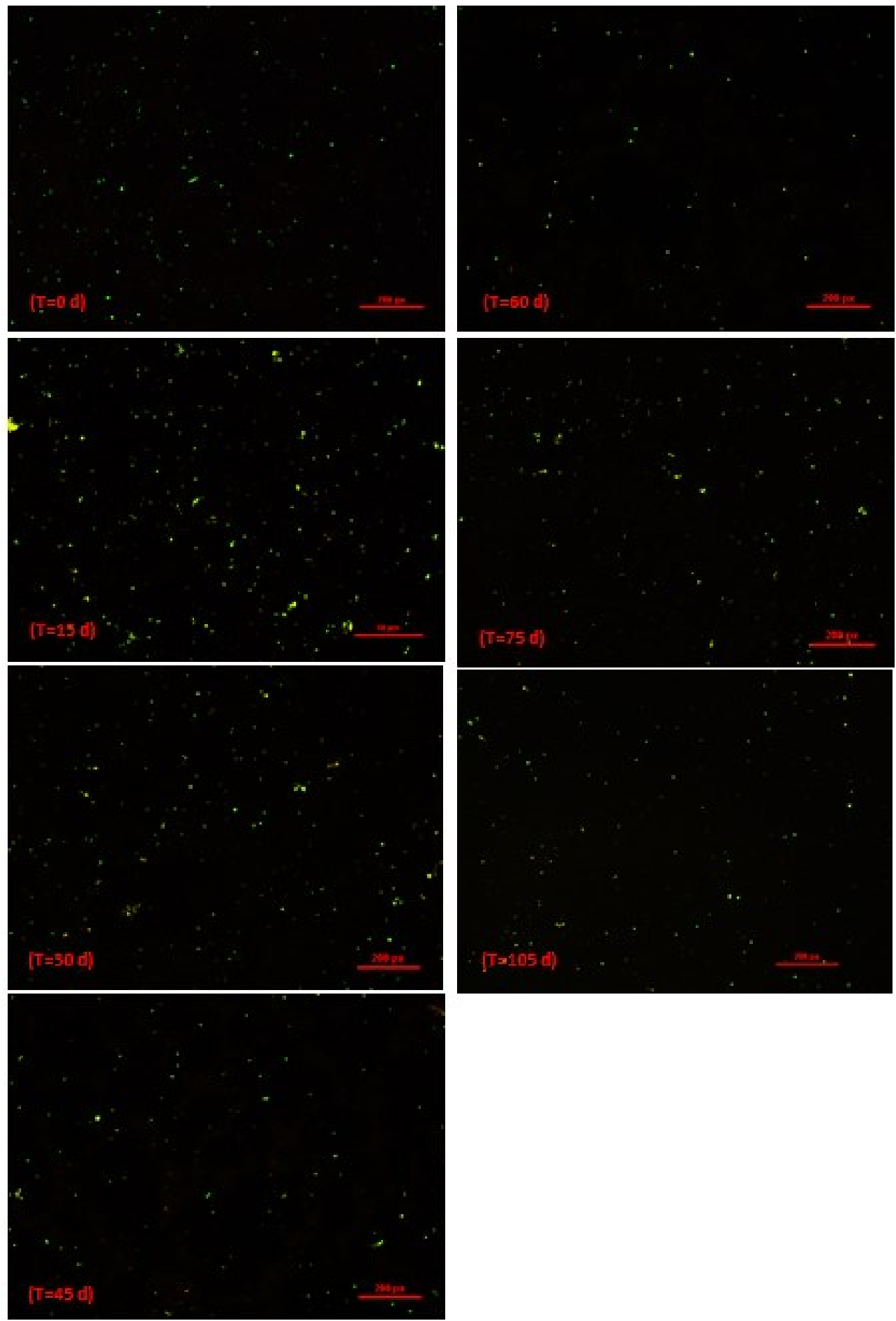

Figure S-2 Live/Dead images of control 1 (the soil reactor that received untreated sludge with no nanoparticles). 

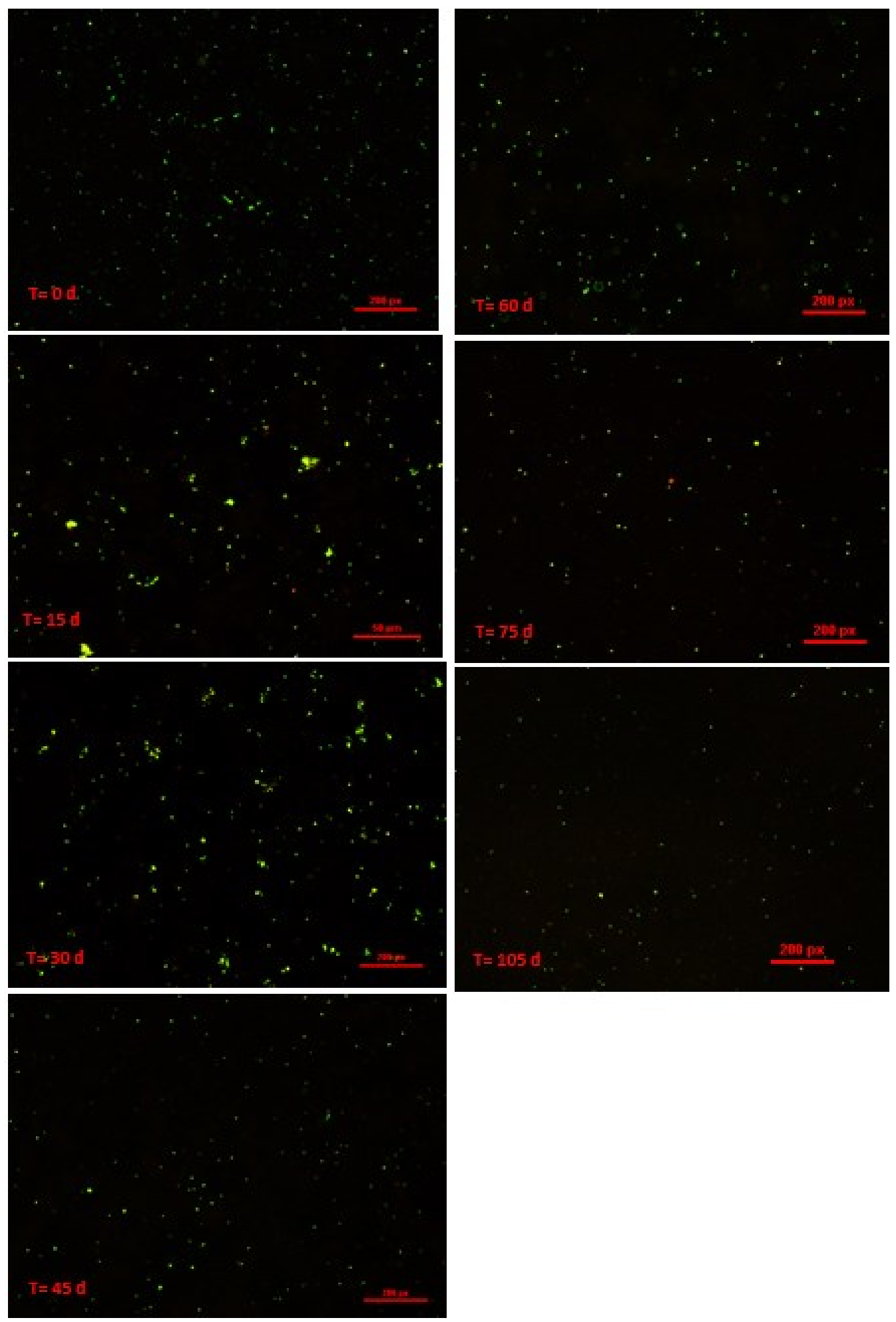

Figure S-3 Live/Dead images of control 2(the soil reactor that received untreated sludge with $2 \mathrm{mg} \mathrm{AgNPs/g} \mathrm{TS} \mathrm{sludge).}$ 


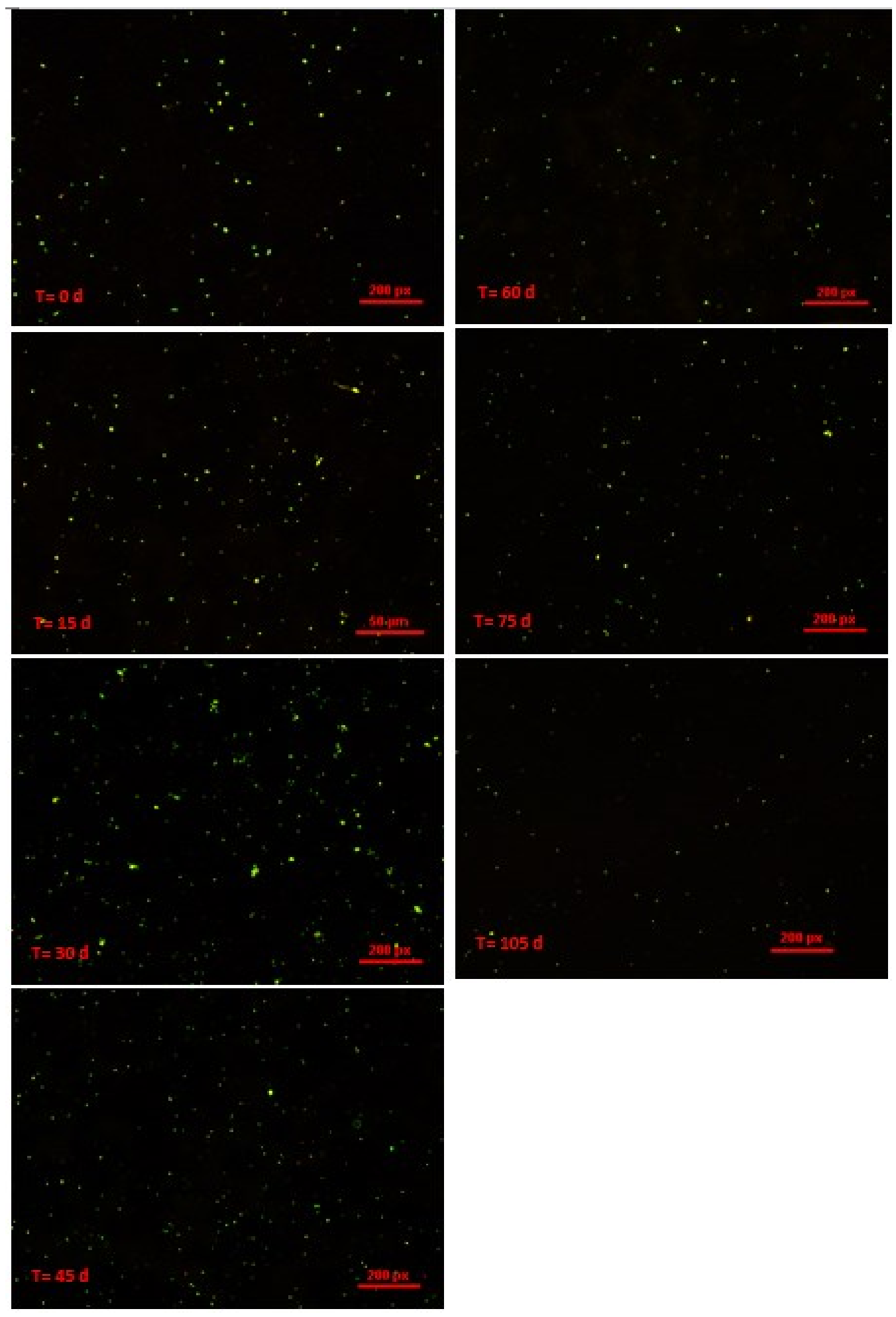

Figure S-4 Live/Dead images of lime reactor (the soil reactor that received lime treated sludge with 2 mg AgNPs/g TS sludge). 


\section{Chapter 8: Conclusions and Future Work}

\subsection{Conclusions}

This chapter summarizes the major conclusions from this thesis and potential areas for further research.

\section{Chapter 4}

- Biogas yield decreased with higher concentrations of AgNPs and CuONPs.

- Bacteroidetes, Firmicutes, and Proteobacteria were the most abundant phyla observed in all the sludge reactors including the control (98\% relative abundance). They had similar average relative abundance trends in all the sludge reactors over time, which suggests that AgNPs and CuONPs had no impact on the phyla.

- Hydrolysis and fermentation bacterial genera OTUs were higher in the reactors with nanoparticles compared to the control while the acetogenic bacterial genera OTUs were lower, indicating that the presence of AgNPs and CuONPs benefitted the hydrolysis and fermentation functional group of bacteria, while inhibiting the growth of the bacterial groups that serve as acetogens.

\section{Chapter 5}

- AgNPs and CuONPs concentrations of $300 \mathrm{mg}$ NPs $/ \mathrm{kg}$ soil decreased the OTUs of the most abundant phyla and genera (phyla at $>90 \%$ relative abundance and genera at $>$ $70 \%$ ), while increased the OTUs of the next most abundant phyla and genera (phyla at $\leq 10 \%$ relative abundance and genera at $\leq 30 \%$ ). This included the decreased relative 
abundance of Proteobacteria (the most abundant phylum in all the soil reactors) in the soil microcosms with high AgNPs and both CuONPs concentrations.

- AgNPs and CuONPs impacted the population of the most abundant and beneficial group of bacteria in the soil as was clear from the reduction in the population of PGPR. This was also supported by the significant reduction of the soil HPC in the microcosms with a higher concentration of AgNPs and CuONPs.

\section{Chapter 6}

- Conditioning chemicals (ferric chloride, alum and polymer) effectively removed AgNPs (at $2 \mathrm{mg} \mathrm{AgNPs} / \mathrm{g}$ TS sludge) from the aqueous phase of sludge, and concentrated them in the sludge solids, which was evident from the results of SP-ICPMS and TEM imaging.

- Different growth patterns of the assessed phyla in the soil were observed over time, corresponding to different conditioners used in sludge conditioning, and of the three conditioners used in sludge conditioning, ferric chloride promoted the growth of all five phyla in the soil, while under polymer treatment, phyla had decreased. However, these differences were not statistically significant.

\section{Chapter 7}

- Lime treatment was found to be efficient in removing AgNPs from the aqueous phase through association with lime molecules.

- Lime stabilization of sludge showed no impact on the average relative abundance of the five assessed phyla in the soil that was amended with this sludge. 


\section{Both Chapters 6 and 7}

- AgNPs in sludge underwent different physicochemical transformations, including aggregation, dissolution and surface coating, which were confirmed from TEM imaging.

- AgNPs at $2 \mathrm{mg}$ AgNPs/g TS of sludge (which corresponds to $20 \mathrm{mg} \mathrm{AgNPs} / \mathrm{kg}$ soil) did not significantly affect the average relative abundance of the five assessed phyla (Acidobacteria, Actinobacteria, Bacteroidetes, Firmicutes and Proteobacteria) in the soil. This was supported by the results of other tested elements such as the soil $\mathrm{pH}$, TOC concentration, HPC and the percentage of the live cells, since there was also no significant impact on these tested factors. Also, the metagenomic analysis showed that among the studied phyla in all the soil reactors Acidobacteria was, on average, the most abundant phylum and Bacteroidetes the least.

\subsection{Thesis contributions}

- The thesis evaluated the transformation of AgNPs and CuONPs during three main different treatments and after the land application of biosolids, thus facilitating a better prediction of their fate and impact in real-life situations.

- The thesis incorporated different advanced methodologies and analyses, including the bacterial community structure to assess the impact of the nanoparticles. Therefore, introducing critical data to better understand their impact on the key bacteria and their critical roles in the anaerobic digestion process of the sludge, and soil fertility functions.

- The research adds important scientific findings to the field of nanoparticle toxicity, and the findings can provide valuable information for the prediction and modelling of 
AgNP and CuONP effects on human health, thus better facilitating the implementation of regulations on nanoparticles products.

\subsection{Future work}

Recommendations for future areas of study include studying the impact of AgNPs and CuONPs on the archaea community structure as these are the primary organisms in the methane production during the anaerobic digestion of sludge. Also, it is important to investigate a wider range of nanoparticle types, concentrations, and characteristic, and establishing their toxicity thresholds, both for biological wastewater treatment processes and after land application of biosolids. Wastewater and sludge treatment processes are advanced engineering systems that can be modified to mitigate nanoparticle toxicity through process design and operational conditions. This requires a comprehensive understanding of the behavior and impact metrics of nanoparticles and can include investigating their activity under different sludge and soil characteristics and considering their pathways in the environment. Other future research could be investigating nanoparticles impact on crops after land application of sludge to address concerns over food risks and safety. 